\title{
Better be prepared
}

Citation for published version (APA):

van Beijsterveld, C. A. F. M. (2020). Better be prepared: transition in perioperative physical therapy care with patients that decided to have surgery because of cancer in the pancreas or liver. [Doctoral Thesis, Maastricht University]. ProefschriftMaken. https://doi.org/10.26481/dis.20200124cb

\section{Document status and date:}

Published: 01/01/2020

DOI:

10.26481/dis.20200124cb

Document Version:

Publisher's PDF, also known as Version of record

\section{Please check the document version of this publication:}

- A submitted manuscript is the version of the article upon submission and before peer-review. There can be important differences between the submitted version and the official published version of record.

People interested in the research are advised to contact the author for the final version of the publication, or visit the DOI to the publisher's website.

- The final author version and the galley proof are versions of the publication after peer review.

- The final published version features the final layout of the paper including the volume, issue and page numbers.

Link to publication

\footnotetext{
General rights rights.

- You may freely distribute the URL identifying the publication in the public portal. please follow below link for the End User Agreement:

www.umlib.nl/taverne-license

Take down policy

If you believe that this document breaches copyright please contact us at:

repository@maastrichtuniversity.nl

providing details and we will investigate your claim.
}

Copyright and moral rights for the publications made accessible in the public portal are retained by the authors and/or other copyright owners and it is a condition of accessing publications that users recognise and abide by the legal requirements associated with these

- Users may download and print one copy of any publication from the public portal for the purpose of private study or research.

- You may not further distribute the material or use it for any profit-making activity or commercial gain

If the publication is distributed under the terms of Article $25 \mathrm{fa}$ of the Dutch Copyright Act, indicated by the "Taverne" license above, 


\section{Better Be Prepared}

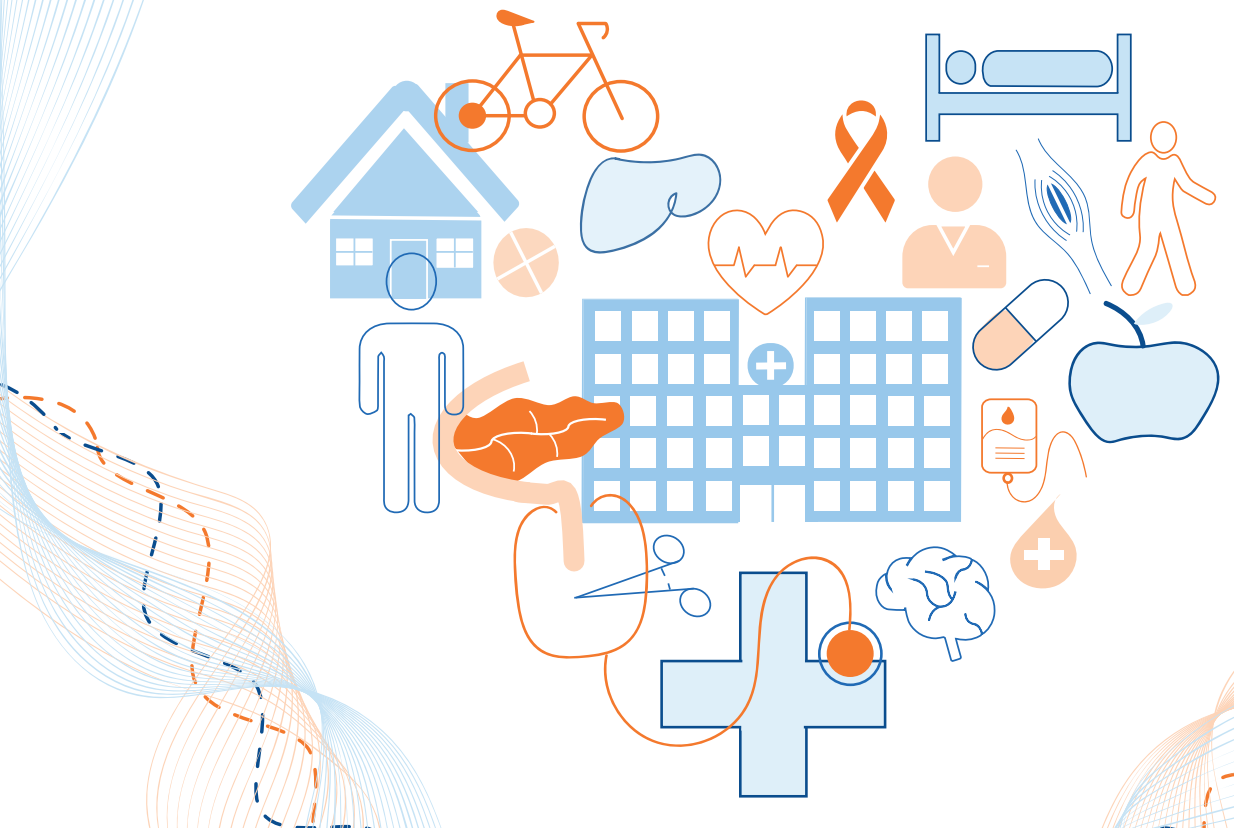

(1)

Transition in perioperative physical therapy care with patients that decided to have surgery because of cancer in the pancreas or liver 


\section{Better Be Prepared}

Transition in perioperative physical therapy care with patients that decided to have surgery because of cancer in the pancreas or liver 
(C) Christel van Beijsterveld, Maastricht 2019

No part of this book may be reproduced or transmitted in any form or by any means, without prior permission in writing by the author, or when appropriate, by the publisher of the publications.

$\begin{array}{ll}\text { Design cover } & \text { Christel van Beijsterveld } \\ \text { Layout } & \text { Christel van Beijsterveld } \\ \text { Printed by } & \text { ProefschriftMaken | www.proefschriftmaken.nl }\end{array}$

ISBN/EAN 978-94-6380-654-1

The research presented in this thesis was conducted at the Department of Epidemiology, research line Functioning, Participation, and Rehabilitation of the Care and Public Health Research Institute (CAPHRI), Maastricht University.

The printing of this thesis was financially supported by Maastricht University, the Department of Physical Therapy of the Maastricht UMC+, Scientific College Physical Therapy (WCF) of the Royal Dutch Society for Physical Therapy (KNGF), ProCare BV, Lode BV, and Maastricht Instruments.

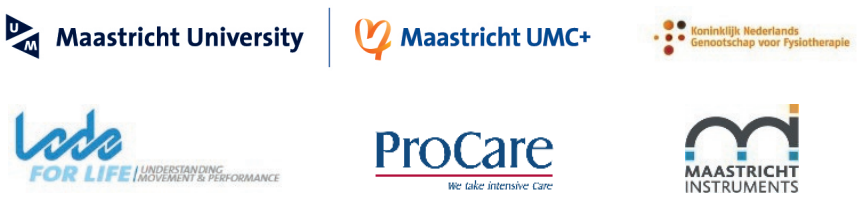




\title{
Better Be Prepared
}

Transition in perioperative physical therapy care with patients that decided to have surgery because of cancer in the pancreas or liver

\author{
DISSERTATION \\ to obtain the degree of Doctor at Maastricht University, \\ on the authority of the Rector Magnificus, \\ Prof.dr. Rianne M. Letschert \\ in accordance with the decision of the Board of Deans, \\ to be defended in public on \\ Friday $24^{\text {th }}$ of January 2020 , at $14: 00$ hours \\ by
} Christel Alice Francisca Maria van Beijsterveld 


\section{Supervisors}

Prof.dr. N.L.U. van Meeteren

Prof.dr. C.H.C. Dejong

Co-supervisor

Dr. B.C. Bongers

Assessment committee

Prof.dr. W.F.F.A. Buhre (chair)

Prof.dr. R. van Hillegersberg (University Medical Center Utrecht)

Prof.dr. S.W.M. Olde Damink

Prof.dr. L.P.S. Stassen

Prof.dr. P.J. van der Wees (Radboud University Medical Center) 




\section{Contents}

Chapter 1 General introduction

Chapter 2 Variation in reported preoperative and postoperative physical therapy management for patients opting for major elective abdominal surgery

Chapter 3 Improvement of preoperative and postoperative performance of physical functioning in patients undergoing elective hepatopancreato-biliary surgery: initiation of a regime transition

Chapter 4 The association between preoperative physical functioning and short-term postoperative outcomes: a cohort study of patients undergoing elective hepatic resection

Chapter 5 Exploring the relation between preoperative physical functioning and the impact of major complications in patients following pancreatic resection

Chapter 6 Personalized community-based prehabilitation in a high-risk surgical patient opting for pylorus-preserving pancreaticoduodenectomy: a case report

Chapter $7 \quad$ General discussion

Chapter $8 \quad$ Valorization

Summary

Samenvatting

Dankwoord 
Een wetenschapper staat niet buiten de maatschappij, het is juist

zijn taak zich daar te bewijzen als gangmaker [....] een bevorderaar van de voortuitgang.

- J. Gottlieb (1762-1814) - 
Chapter 1

Introduction 



\section{PREFACE}

In 1946, prehabilitation was mentioned for the first time in a scientific publication, which focused on the improvement of the physical fitness level of young recruits of the British Army [1]. Approximately 12,000 recruits followed the program consisting of exercise training, education, and nutritional support, of which $85 \%$ of the recruits improved on physical and mental parameters [2]. Later on, in 1985, prehabilitation was used in the field of sports medicine [3], and Topp et al. [4] proposed a framework for enhancing functional capacity of patients before admission to the intensive care unit to improve coping to stressful events in the 21 st century. Moreover, also other groups were proposing new models to enhance perioperative care. Kehlet [5] introduced a multimodal approach in 1997 consisting of education, attenuation of stress, pain relief, exercise, nutrition, and growth factors, to accelerate postoperative recovery, called fast-track surgery. At the same time, other surgeons were focusing on the surgical stress response from a metabolic and endocrine viewpoint [6]. These efforts have led to the introduction of the enhanced recovery after surgery (ERAS) program in 2001 by a group of academic surgeons in Europe to improve postoperative outcomes [6]. In 2005, an ERAS clinical care protocol was published introducing a multidisciplinary and multimodal approach substantiated with scientific evidence [7].

Nowadays, the perioperative care process from diagnosis, indication for surgery, admission, surgery, and postoperative recovery is still adapting. Perioperative care is moving towards the concept of P4 health. The four P's represent perioperative care that is Predictive, Preventive, Personalized, and Participatory [8]. A P4 perioperative care approach aims for optimal vital functioning before, during, and after hospitalization associated with the major life event of a substantial surgical intervention in the thorax, abdomen, or large joints as the knee or hip. Hereto, the mission of this thesis was to guide the transition in perioperative physical therapy care, from a more or less reactive approach towards proactive $\mathrm{P} 4$ perioperative physical therapy care. More specifically, perioperative physical therapy care that will focus on maintaining vital physical functioning during a major life event episode in patients undergoing elective hepatic or pancreatic resection. To achieve this mission, the aim was to implement several evidence-based so-called 'point' solutions - state-of-the-art scientific research, theories, and perspectives about proactive perioperative physical therapy management - in the subsystem of patients and their supportive physical therapy care. At the same time, these 'point' solutions were validated in an ecological way in the real-life context of daily practice. 


\section{Reading journey}

Societal challenges and new technological developments lead to transitions in different fields (e.g., energy transition, health transition, technology transition). In this general introduction, a helicopter view about how to address transitions with a mission-driven research and innovation approach is introduced. The approach is applied on the transition in P4 perioperative (physical therapy) care, which is the central theme of this thesis. An intermezzo-box is used to provide the reader with in-depth background information on specific topics.

\section{MISSION DRIVEN APPROACH FOR THE TRANSITION IN PERIOPERATIVE CARE}

The concepts of health and health care are currently in transition due to demographical changes and coinciding rapid changes in medical technologies. The proportion of people aged 65 and older is rapidly increasing globally. Current estimates expect that by $2040,26.5 \%$ of the Dutch population will be older than 65 years [9]. Furthermore, one out of eleven Dutch adults will be above 80 years in 2040 [9]. Providing optimal care for elderly in order to preserve independent functioning and quality of life is challenging due to the presence of complex comorbid geriatric problems and syndromes, the related complex clinical management, the availability of health care services, and rising health care costs [10]. Coinciding with the ageing population, anesthetic and surgical techniques have improved tremendously throughout the last decades [11]. Hereto, major surgery is more often performed in elderly patients. Specifically, in these patients, the challenge is to maintain vital functioning throughout the episode of a major life event, such as hospitalization and undergoing major surgery. To address the societal challenges and innovations, and thereby leading a transition, a mission-oriented research and innovation policy has been proposed by Mazzucato in 2018 (see Box 1.1) [12].

A mission-driven approach involves tackling problem-specific societal challenges by focusing on creating system-wide transitions across different domains (e.g., health care, technology, policymaking, economy) [13]. The central mission for health and care in the Netherlands is "by 2040, all Dutch citizens will live at least five years longer in good health, while the health inequalities between the lowest and highest socio-economic groups will have decreased by 30\%" [14]. Within this mission and its consequently warranted transition, health is more and more seen as an important prerequisite for people's daily functioning in their own living context. This active daily functioning is considered to facilitate physical, mental, and social-health and wellbeing and is thereby already preventive in itself [14]. 
Box 1.1. Challenge-driven policy

Each individual faces numerous challenges in daily life that vary for example from independent physical functioning in their community or using digital technologies, towards having access to clean drink water. Clustering these individual's challenges leads to societal challenges (e.g., health and wellbeing concerns). Societal challenges are complex, stubborn, and need interaction with different actors. The challenges ask for more knowledge and understanding, new solutions, and changing policies at different levels (local, regional, national, and international). Adjacent to these societal challenges, people are dealing with new developments and changing beliefs in technology, economy, and society. These developments in different domains interact with each other and reinforce each other [18]. For instance, developments in technology do not only include innovations in technological performance, but also influence economic, social, and cultural systems, as it requires parallel innovations in policy and changes with regard to the 'behavior' of the regulatory context and the user $[19,20]$. Thus, both societal challenges and new technological developments lead to transitions in different fields (e.g., energy transition, health transition, technology transition).

A mission-driven approach starts from a grand societal challenge. From here, various missions can be linked. The grand challenges for the Netherlands include climate, migration, education, and health and well-being concerns. The aim of the mission-driven policy is to turn the grand challenges into different missions across multiple sectors and actors. Missions require collaboration between citizens, governments, entrepreneurs, and researchers to align on technologies and socio-economic policies that can fulfill societal challenges that are 'smart', 'inclusive', and 'sustainable' [14, 21]. Each mission contains a portfolio of different research and innovation projects that are bottom-up initiated out of relatively small niches, and driven by changing value patterns. Examples of these innovation projects are, among others, the recent reconceptualization of "Health" by Huber et al. [22], "Gemeenschapskracht" [23], a new national health research infrastructure for personalized medicine (Health-RI), and artificial organs like "NeoKidney" [24]. 
Challenge: vital functioning citizens during major life events in a healthy economy

The mission-oriented research and innovation policy framework by Mazucatto [12] can also be applied in perioperative care. Maintaining daily physical functioning before, during, and after a major life event, such as surgery is a grand challenge for people (see Figure 1.1, grand challenge). Frail patients opting for major surgery experience a decrease in their physical functioning due to their hospitalization and surgery. Therefore, the collaborative decision for surgery by patients and health care providers is difficult and complex, as not every patient will benefit from surgery. It is hard to predict the benefit/loss ratio for an individual patient. It involves the discussion of the beneficial effects of the surgical treatment, other treatment options, the risk for complications, future perspectives, and patient preferences [15]. If patients opt for the surgical procedure and its associated hospitalization, prevention of a complicated (post)operative course is essential to swiftly return to the ability to perform activities of daily life independently, as well as future societal participation that is becoming more important nowadays [16].

\section{Mission: from reactive to proactive in perioperative care}

This mission aims to provide solutions for the grand challenges, but should be clear and welldefined. Maintaining daily physical, mental and social functioning before, during, and after a major life requires a proactive strategy focusing on functioning instead of a reactive, sort of 'sit wait and see' strategy. For patients undergoing major elective surgery, this implies more patient-centered and personalized care, meaning that it facilitates the empowerment of the patient in decisionmaking, monitoring, and management of health status and daily functioning in the patient's own dynamic context [17]. Therefore, the overall mission for perioperative care is to deliver proactive care for all and preventive for the high-risk patients (see Figure 1.1, Mission).

\section{Research and innovation}

The mission to transfer from reactive to proactive perioperative care requires a transition in the ongoing health care regime and policy within the current culture (prevailing perspectives, behavior), practices (procedures and protocols), and structures (health care facilities). Consequently, this creates a window of opportunity for research and innovation projects at a niche level performed by multiple actors (see Figure 1.1, research and innovation projects) [25]. These research and innovation projects, combined with cross-sector and cross-actor innovations (e.g., policy, government, food, technology) need to align and interact with each other across the entire transition to succeed in the described mission and thereby achieve an overall impact in perioperative care. 
A. Grand challenge

Vital functioning citizens during major life events in a healthy economy

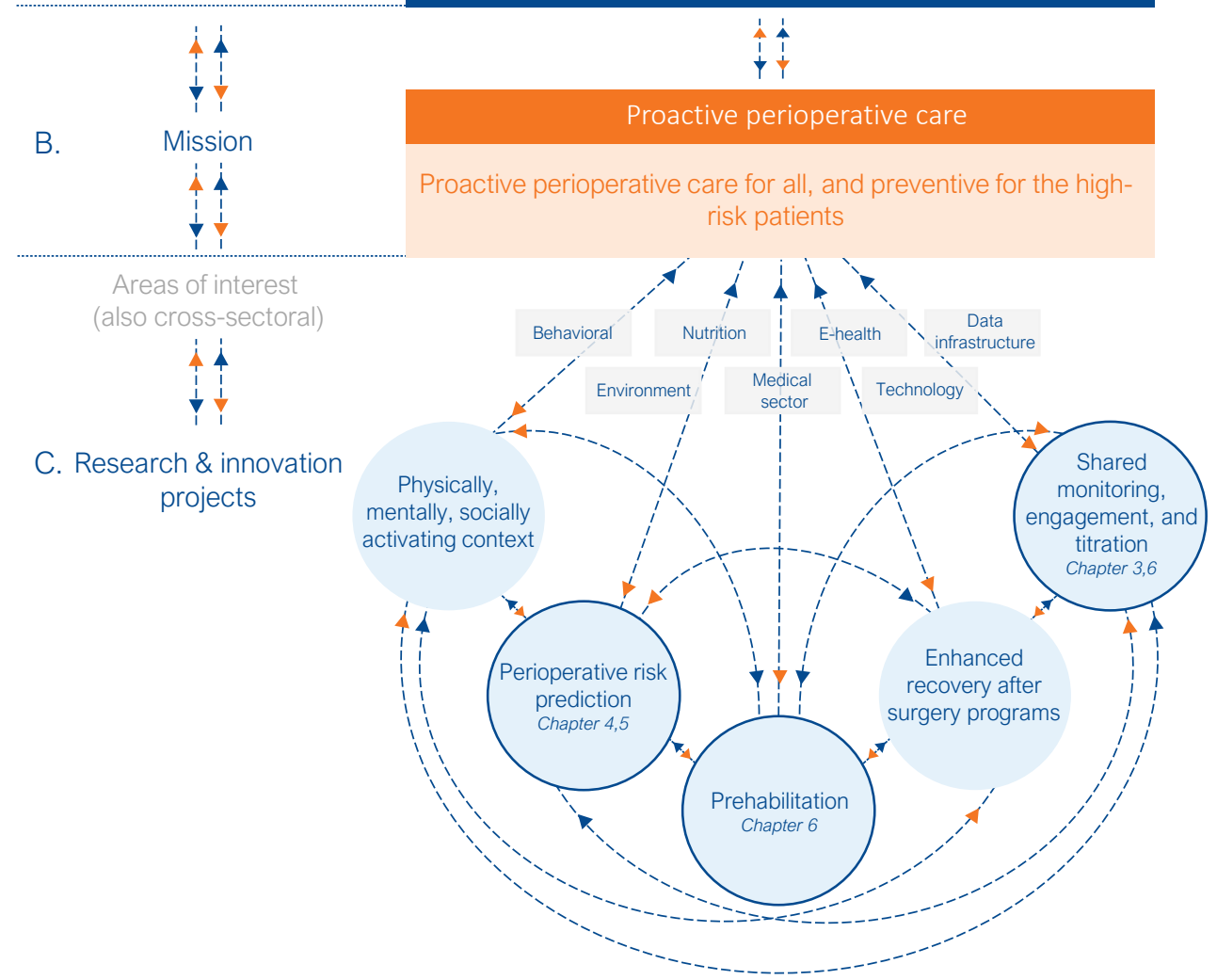

Figure 1.1. From the grand challenge to a mission for research and innovation projects in perioperative care.

A. The grand challenge is to aim for vital functioning citizens during major life events (elective hepatic or pancreatic surgery in this thesis) in a healthy economy.

B. The mission that supports this grand challenge is to work towards proactive perioperative care with preventive strategies for high-risk patients.

C. Different bottom-up research and innovation projects will, in collaboration with the different actors from various areas of interest (also cross-sectoral), guide and drive the mission. Regarding perioperative care, this means different research projects involving perioperative risk prediction, prehabilitation, and shared monitoring, engagement, and titration. The research projects should be intertwined with each other and connected with the different areas of interest to achieve an overall impact on the mission.

This figure is adapted and modified from Mazzucato [12] and the Knowledge and Innovation agenda 2020-2023 of the Top Sector Life Science \& Health [14]. 
With the transition in perioperative care, from a reactive curative approach to a proactive preventive approach, it is advised to design research and innovation towards a proactive predictive, preventive, personalized, and participatory (P4) health approach. Perioperative P4 perioperative care is advocated to be:

1. predictive (e.g., preoperative screening of patients to predict a patient's surgery-related risks);

2. preventive (e.g., patients who are at risk are offered temporary lifestyle interventions as physical exercise training, nutritional support, and psychological support to enhance coping with the consequences of hospitalization and surgery and enhance postoperative recovery);

3. personalized (e.g., tailor-made inventions for the individual patient leading to a high patient adherence and improved treatment outcome); and

4. participatory (e.g., encourage patients and their (in)formal caregivers to actively participate in their treatment through active self-care and self-management) [16, 26-27].

\section{WINDOW OF OPPORTUNITY FOR ELECTIVE PANCREATIC AND HEPATIC RESECTION}

Cancer in the hepato-pancreato-biliary (HPB) tract involves some of the most prevalent, as well as most lethal causes of cancer deaths of people worldwide, thereby constituting a considerable public health problem. Pancreatic cancer is ranked as the ninth most common cancer in the Netherlands, with an incidence of 2,947 newly diagnosed patients in 2018 and accounting for about $6.8 \%$ of cancer-related deaths in the Netherlands [28]. The largest part (74.6\%) of hepatic cancer diagnosis in 2018 in the Netherlands comprised metastases in the liver from primary tumors in other organs, most commonly of colorectal origin (67.0\%) [29]. Primary liver cancer is the sixth most common cancer and is the third most lethal cause of cancer deaths worldwide [31]. There is wide geographic variability in the incidence of primary liver cancer [30] due to the prevalence of hepatitis B. Europe accounted for $9.8 \%$ of all new cases of primary liver cancer in 2018, which is relatively low compared to its incidence in Asia (72.5\%) [28].

Surgical resection of the tumor is the primary treatment modality in patients diagnosed with cancer in the HPB tract. Although major advances in surgical techniques and anesthesiology procedures have led to improved perioperative management and surgical outcomes, HPB procedures remain highly complex. Hence, HPB surgery is accompanied by a relatively high rate of postoperative morbidity and mortality. In hepatic surgery, morbidity rates range from $8.9 \%$ to $22.0 \%$, whereas mortality rates range from $1.0 \%$ to $5.0 \%$, which is dependent on type of surgery 
and extent of hepatic resection in the Netherlands [31]. Morbidity and mortality rates within 30 days following pancreatic surgery in the Netherlands are about $60.0 \%$ and $2.9-3.5 \%$, respectively [32].

The challenge: adapting to treatment-related stress while maintaining vital functioning Maintaining vital functioning before, during, and after major surgery, is challenging among patients. For patients, the pathology, surgery, the accompanied hospitalization, and - sometimes - (neo)adjuvant treatment is a stressful and physically and socially demanding experience. Especially frail patients experience a loss of independent physical functioning following surgery and hospitalization, which influences their ability to perform activities of daily life [33-35]. Due to the ageing population, health care professionals are increasingly confronted with geriatric syndromes in patients with cancer in the HPB tract. These syndromes are challenging in the light of preventing postoperative complications and their consequences. As a result, patients experience pathophysiological, contextual, and personal stressors throughout the complete perioperative continuum. Adaptation to stressors to maintain homeostasis is called allostasis, involving physiological responses that lead to temporary bodily adaptations to cope with the stressor [36]. The process of allostasis appeals to physiological reserves. Patients with an adequate physiological reserve capacity (allostatic reserves) are able to adapt to the treatmentrelated stress. The latter reduces the consequences of the allostatic load, leading to a faster return to homeostasis and more favorable surgical outcomes [37, 38]. On the contrary, patients with less physiological reserve capacity are less able to adapt to stressors and are therefore at a greater risk for poor outcomes following surgery [38] (see Figure 1.2).

\section{Preoperative stressors}

Cancer has an impact on the patient's physical, psychological, and social well-being. It is associated with profound metabolic and neurochemical alterations, such as altered protein metabolism, adipose tissue loss, and inflammation. This can lead to progressive wasting of muscle mass, making patients prone to become cachectic $[39,40]$. The loss of body mass is the main criterion in the definition of cancer cachexia [40] and is present in $83 \%$ of patients with pancreatic cancer [41]. Besides the pathophysiology of cancer cachexia, disease symptoms and anti-tumor therapies (e.g., chemo-, radio-, immunotherapy) hamper adequate food-intake (e.g., vomiting, nausea, early satiety). This influences the clinical course of the disease, the patient's physical performance, and the patient's quality of life, especially in frail elderly [39, 40, 42]. Frailty can be defined as a diminished adaptive capacity and increased vulnerability to cope with events 
(e.g., surgery, chemoradiation, hospitalization), that are stressors for the physiological system $[38,43]$. Several studies in patients undergoing HPB surgery have shown that the presence of frailty (clinical and morphometric parameters) is associated with an increased risk for perioperative morbidity and mortality $[44,45]$.

Although surgery is the curative treatment for cancer in the HPB tract, some patients will receive advanced systemic treatments preoperatively to reduce the tumor load. In patients with nonmetastatic locally advanced pancreatic cancer, neoadjuvant treatment with chemo- and radiotherapy might increase the patient's chance of being eligible for surgical exploration, resection, and survival [46]. However, neoadjuvant chemotherapy itself can also lead to loss of skeletal muscle mass and adipose tissue, both in patients with resectable pancreatic cancer [47] and in patients with resectable colorectal liver metastases [48]. The age-related loss of skeletal muscle mass and density, called sarcopenia is associated with chemotherapy toxicity [49] and adverse outcomes after surgery for HPB malignancies [50, 51]. The study by Sandini et al. [52] reported that an increase in muscle tissue during neoadjuvant chemotherapy was associated with a higher likelihood for resection in patients with pancreatic cancer. In contrast, patients with a decrease in lean body mass had a higher likelihood for surgical exploration and no resection [51]. There was no particular intervention, like exercise to stimulate the increase in muscle tissue in this study; however, a reduction of cancer-related inflammatory factors secondary to chemotherapy could be a potential explanation of the decrease in protein wasting [52].

Besides the physiological consequences of cancer and patient's related comorbidities, cancer and its treatment also influence a patient's mental and social health. The individual response to cope with stress caused by the disease will impact patient's psychological state and quality of life. The prevalence of anxiety and depression in patients with cancer is twice as high compared to people without cancer [53]. Postoperative quality of life and physiological surgical outcomes are associated with preoperative anxiety, depression, and low self-efficacy [54]. Hence, psychological monitoring and management should be considered to offer to patients throughout the continuum of care. However, it is still debatable what type of coping strategies can be best provided to each individual patient [55]. 


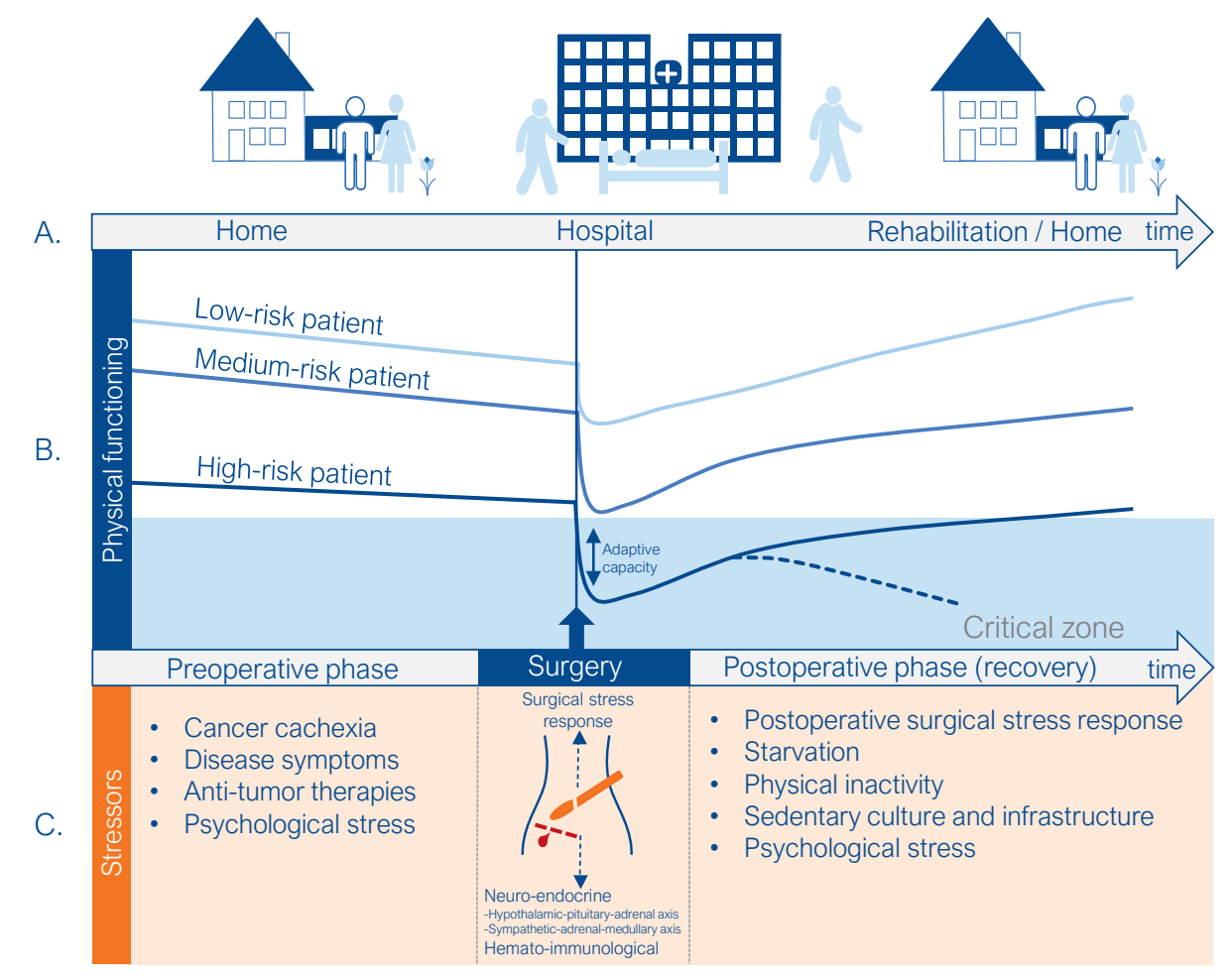

Figure 1.2. Perioperative stressors and its relationship with a patient's physical functioning throughout the patient journey.

A. The patient journey, starting from the patient's living context (home), to hospital admission, and returning back to the living context (home).

B. A patient's preoperative physical functioning is related with postoperative recovery of physical functioning. Patients with a low preoperative level of physical functioning ( - high-risk patient) are likely to enter a critical zone ( physical functioning and adaptive capacity below minimum level). Consequently, these high-risk patients have an increased risk in developing postoperative complications and might experience an inadequate recovery of physical functioning compared to patients with a higher physical fitness level ( — low-risk patient).

C. A patient's level of physical functioning is influenced by stressors ( $)$ in the pre-, peri-, and postoperative phase.

This figure is adapted and modified from Hulzebos \& Van Meeteren [26, 27].

\section{Intraoperative stressors}

In 1920, Cuthbertson [56] was the first who observed the increased loss of metabolites in urine in trauma patients with fractures of the long bones of the lower extremities, representing a breakdown of lean tissue, particularly skeletal muscle. The early observational descriptive studies by Cuthbertson were further investigated, and these studies looked in more detail to the body's stress response following surgery. These efforts have led to further improvements in anesthesia, 
analgesia, and surgical procedures in order to reduce the surgical stress response and improve surgical outcomes [56]. The surgical incision leads to local injury of tissue, blood vessels, and afferent nerves. This will lead to a stress response, which is accompanied with wide metabolic and hormonal changes to limit tissue damage, inhibit infections by organisms, and activates repair processes [57]. Two main systemic stress responses are the neuro-endocrine and haematoimmunological responses [58, 59] (see Figure 1.3 and Box 1.2). Overall, the surgery-induced stress response leads to alterations in protein, lipid, and carbohydrate metabolism. An adequate aerobic capacity and skeletal muscle mass are needed to cope with the increased oxygen demand and metabolic rate (e.g., surgery-induced protein catabolism). The increase in the body's oxygen demand depends on the type and duration of the procedure. However, in major intraabdominal surgery it has been estimated that it is associated with an increase in oxygen demand of $40 \%$ or more $[60,61]$.

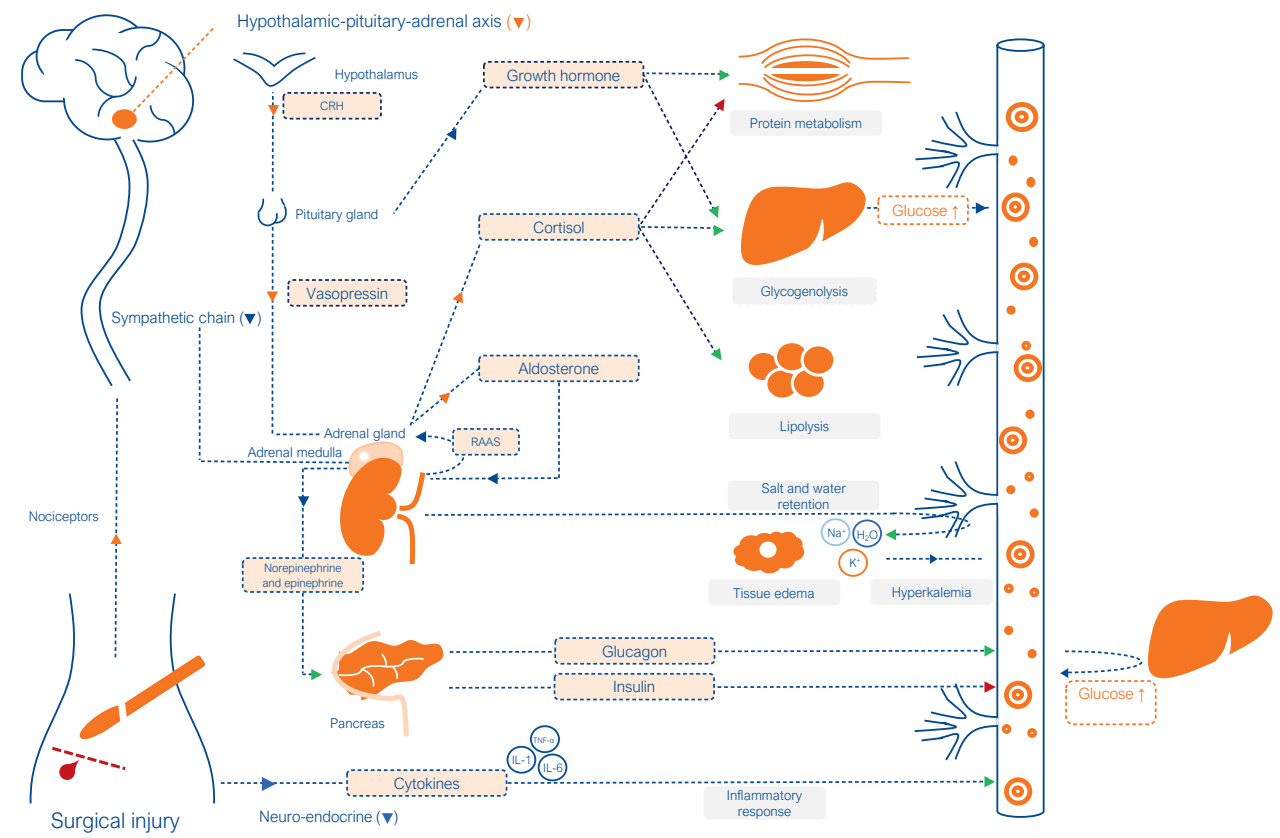

Figure 1.3. The relationship between surgical injury and the multisystem effects of the surgical stress response.

Abbreviations: $\mathrm{CRH}=$ corticotropin-releasing hormone; $\mathrm{H}_{2} \mathrm{O}=$ =water; IL-1=interleukin-1; IL-6=interleukin6; $\mathrm{K}^{+}=$potassium; $\mathrm{Na}^{+}=$sodium; TNF- $\alpha=$ tumor necrosis factor alpha; RAAS=renin-angiotensinaldosteron system. 
Box 1.2. Two main systemic stress responses to surgery

\section{Neuro-endocrine response}

The neuro-endocrine response is the response in which the hypothalamic-pituitary-adrenal axis and sympathetic-adrenal-medullary axis are activated. Afferent signals from the injury site will send a signal to the hypothalamus, resulting in an activation of the hypothalamicpituitary-adrenal axis. The hypothalamus will release several hormones that control the pituitary gland [59]. The pituitary gland will release growth hormone - e.g., stimulating protein anabolism and glycogenolysis in the liver [62] - and will control the adrenal gland by releasing vasopressin. The stimulation of the adrenal gland induces the release of cortisol which has complex metabolic effects on proteins, carbohydrates, and lipids [59]. It leads to protein catabolism: after major surgery, the loss in lean body mass might be up to 0.5 $\mathrm{kg} /$ day, depending on the type of surgery and preoperative nutritional status of the patient [57]. Additionally, cortisol also stimulates lipolysis, promotes liver glycogenolysis, and inhibits the use of glucose by cells leading to an increase in blood glucose levels inducing hyperglycemia [59]. Furthermore, stimulation of the adrenal glands leads to secretion of aldosterone resulting in retention of sodium and water and increased potassium secretion in tissues. As a result of the stimulation of the sympathetic-adrenal-medullary axis, the adrenal medulla will release norepinephrine and epinephrine resulting in cardiac effects (tachycardia, hypertension) and stimulation of the pancreas following inhibition of pancreatic beta-cell insulin secretion and glucagon stimulation [59]. This leads to increased blood glucose inducing hyperglycemia. Hyperglycemia is an independent risk factor for adverse outcomes after surgery and is commonly called the 'the diabetes of injury' [63].

\section{Hemato-immunological response}

The hemato-immunological response is a complex biochemical cascade resulting from the surgical trauma. Cytokines are produced at the injury site by fibroblasts, macrophages, and endothelial cells, inducing a pro-inflammatory state [58]. The main cytokines responsible for inducing systematic changes in the acute-phase response after surgery are interleukin-1, interleukin-6, and tumor-necrosis-factor-a [59]. Furthermore, they enhance the release of cortisol, C-reactive proteins, and complement and coagulation proteins [64]. 


\section{Postoperative stressors}

After surgery, the patient is admitted to the recovery room with tubes and drains, where the patient awakens from the anesthesia. This is in great contrast with the hours before surgery, in which the patient was able to walk and had no incision or intravenous fluid. From this moment, recovery from surgery has started, which does not only include recovery in physical domains, but also recovery in psychological and social domains [65-67]. Recovery can be divided into three phases: early, intermediate, and late, in which each phase has different outcomes in different domains (see Figure 1.4) [68]. For instance, the early phase - from the recovery room to the surgical nurse ward - is characterized by biological and physiological changes, whereas the intermediate phase consists of the regaining functional independency and discharge to home [68]. The late phase consists of return to normal activities and being able to perform activities of daily life independently [68]. In each phase, however, patients can be exposed to stressors (pain, complications, physically inactive infrastructure, difficulties in intake), which may all influence postoperative recovery of physical functioning depending on the precipitation of each individual patient.

In the early phase of recovery, there is a major role for the anesthesiologist to further reduce the stress-response to surgery by monitoring circulation, consciousness, oxygen saturation, pain control, and fluid balance. The extent of the surgical stress response depends on the precipitation of the patient, the type and invasiveness of surgery, and leads to organ-specific stress responses. Patients should be able to adapt to the stress responses to enable postsurgical recovery. For instance, in the respiratory system, pain of the incision, mechanical ventilation, analgesia, and immobility leads to an increased risk for pulmonary complications due to changes in ventilation patterns, alterations in gas exchange, and deterioration of the diaphragm [69]. Hereto, awareness and acknowledgement of physiological changes in the postoperative period are important for adequate acting on the response avoiding complications, and to impair functional decline. 


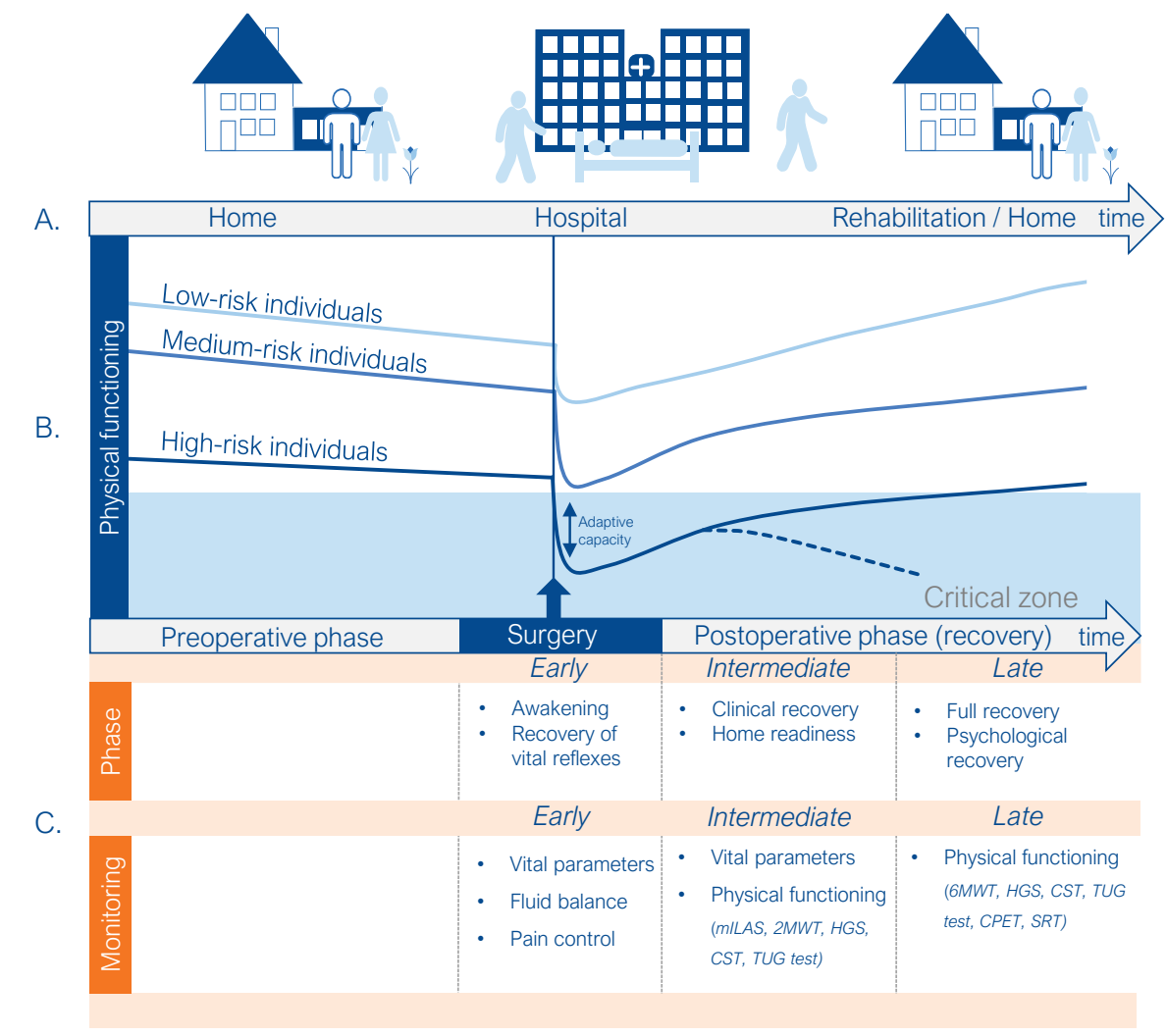

Figure 1.4. Monitoring a patient's recovery of physical functioning throughout the postoperative phase. A. The patient journey, starting from the patient's living context (home), to hospital admission, and returning back to the living context (home).

B. A patient's preoperative physical functioning is related with postoperative recovery of physical functioning. Patients with a low preoperative level of physical functioning ( - high-risk patient) are likely to enter a critical zone ( physical functioning and adaptive capacity below minimum level). Consequently, these high-risk patients have an increased risk in developing postoperative complications and might experience an inadequate recovery of physical functioning compared to patients with a higher physical fitness level ( — low-risk patient).

C.Postoperative recovery of physical functioning can be divided in to three phases: early, intermediate, and late. In each phase, several instruments can be used to monitor a patient's recovery of physical functioning.

This figure is adapted and modified from Hulzebos \& Van Meeteren [26, 27]. 
In the intermediate phase - when the patient is discharged to the surgical nurse ward - the goals are cessation of symptoms (nausea, vomiting, pain) and regaining independent physical functioning (the ability to perform activities of daily life independently) [68]. However, a significant stressor for the patient is the hospital environment. The infrastructural, cultural, and professional context is physically, mentally, and socially sedentary and arranged to treat and/or manage the medical illness; however, it provokes physically inactive behavior, which is a serious side effect [35]. Physical inactivity and bed rest lead to muscle atrophy and diminished aerobic capacity, which both lead to a rapid and profound functional decline. Although the dangers of bed rest concerning the physiological system were already described in 1947 [70], patients still spend an average of $83 \%$ of hospital stay in bed [71] with all its consequences. Twenty-eight days of bed rest in combination with hypercortisolemia in six young healthy subjects resulted in a loss of $6 \%$ $(1.4 \pm 0.1 \mathrm{~kg})$ loss of lean muscle mass [72]. These changes were observed in well-nourished subjects, and the magnitude is consistent with clinical paradigms [72]. Especially in older patients, bed rest related to hospital stay results in a diminished ability to perform basic activities of daily life, like transfers and walking [73-75]. The loss of independent physical functioning is a common side effect of hospitalization and is also called hospitalization-associated disability [35]. Approximately $30 \%$ of patients older than 70 years develop a loss of ability to adequately perform activities of daily life during hospitalization [35]. Consequently, not every patient is able to reach baseline levels of physical functioning after hospitalization and surgery. Lawrence et al. [33] followed 372 patients during long-term recovery of independent physical functioning after major open abdominal surgery and reported that less than $50 \%$ of patients had recovered back to baseline levels after six months. Therefore, monitoring a patient's recovery of physical functioning by measuring skeletal muscle mass, functional mobility, and aerobic capacity during and after hospitalization is important to anticipate early on the detrimental effect of hospitalization and the stress of surgery.

\section{TRANSITION TOWARDS P4 PERIOPERATIVE PHYSICAL THERAPY CARE}

Patients are exposed to multiple stressors throughout the perioperative journey. Some of these stressors are non-modifiable. However, others might be modifiable and provide a window of opportunity to develop research and innovation projects for preoperative optimization, thereby aligning on the mission for optimal perioperative care [28]. P4 perioperative care aims to improve the anticipation and coping with an upcoming stressor in the process of care. The concepts and programs of prehabilitation, ERAS, and fast-track surgery are all supportive to P4 perioperative care. 


\section{Predictive}

Perioperative risk is the product of the likelihood for adverse outcomes and its consequence after surgery. The likelihood is the probability of occurrence of any perioperative event that has an effect on a patient's physical functioning. The aim of preoperative risk assessment is to quantify the likelihood for a postoperative event, and is a prerequisite for clinical reasoning and shared decision-making concerning the choice and use of prophylactic, surgical, and anesthetic interventions [26, 27]. Risk assessment is a complex but necessary effort to establish whether a patient has sufficient adaptive capacity to cope with stressors throughout the perioperative journey [76]. Screening on potential risk factors in HPB surgery should include the assessment of comorbidities, frailty, sarcopenia, malnutrition, and physical fitness and physical functioning [77].

A patient's preoperative physical fitness and physical functioning level is associated with postoperative outcomes in patients undergoing major abdominal surgery [78-80]. As outlined above, patients should be able to meet the increase in oxygen demand during and after surgery. Objectively measuring a patient's preoperative aerobic capacity by cardiopulmonary exercise testing (CPET) reflects the patient's ability to meet this increased oxygen demand. CPET is the gold standard test to determine a patient's aerobic capacity by measuring the body's peak oxygen uptake during a maximal effort ( Vo $_{2 \text { peak }}$ ), whereas the oxygen uptake at the ventilatory anaerobic threshold (VAT) serves as a submaximal indicator of a patient's aerobic capacity [81-83]. In patients undergoing hepatic or pancreatic resection, performing preoperative CPET to assess aerobic capacity has been reported to be valuable in predicting the risk for postoperative morbidity, mortality, and length of stay [84-89]. A Vo $_{2 \text { peak }}<15 \mathrm{~mL} / \mathrm{kg} / \mathrm{min}$ and an oxygen uptake at the VAT $<9-11 \mathrm{~mL} / \mathrm{kg} / \mathrm{min}$ in patients undergoing HPB surgery have been associated with an increased risk for postoperative complications $[82,90]$. However, also the use of more practical performance-based tests to evaluate a patient's preoperative level of physical fitness (e.g., aerobic capacity, muscle strength) and physical functioning (e.g., functional mobility) should be considered, as those tests are easy to perform and therefore more easy to frequently monitor physical status throughout the perioperative journey. Nevertheless, these latter tests have limited predictive value [91]. In patients undergoing elective abdominal surgery, performance-based tests such as the timed up-and-go test, stair-climb test, and six-minute walk test are identified as feasible, as well as associated with postoperative morbidity [79, 91-93]. In addition, preoperative assessment of nutritional assessment is also advocated, as a poor nutritional status is related with adverse postoperative outcomes [94]. Several practical screening tools exist to identify 
malnourished patients in an early stage, such as the malnutrition universal screening tool and short nutritional assessment questionnaire [94]. Furthermore, sarcopenia can be detected with preoperative computed tomography scans [95]. Although an overall multidisciplinary riskprediction tool does not yet exist, it is advocated, based on the above-mentioned issues. Patients and health care professionals should jointly perform a preoperative evaluation in multiple domains and reflect on its prognostic implications in the decision for surgery and for other (co)interventions during the entire perioperative period.

\section{Preventive}

The preoperative period provides a window of opportunity for elective surgical patients, especially for those classified as high-risk patients, in order to decrease their risk for adverse postoperative outcomes by optimizing their reserve capacity before surgery [26, 27, 96]. However, preoperative optimization is challenging, as the period for prophylactic interventions might be limited as patients are frequently scheduled within four weeks after diagnosis, especially in patients with cancer in the HPB tract. Although time is limited, it is an ideal opportunity and a teachable moment to improve a patient's reserve capacity, focusing on physical exercise training and physical activity, nutritional support, and psychological support [97]. In patients undergoing oncological HPB surgery, the introduction of prophylactic interventions such as preoperative physical exercise training and nutritional support has been shown to improve preoperative physical fitness, prevent preoperative deterioration in nutritional status, reduce postoperative complications, and decrease length of hospital stay [96, 98, 99]. Additionally, preoperative optimization of a patient's reserve capacity might decrease the postoperative dip in physical functioning during hospitalization. Due to physical exercise training, aerobic capacity and muscle mass will increase to improve coping with surgical stress, thereby enabling the patient to perform early mobilization after surgery [100]. A prerequisite to further prevent a hospital-induced decline in physical functioning is to create a hospital culture and infrastructure in which patients are stimulated to be physically active and have the ability to participate in activities of daily life [26, 27].

\section{Personalized}

Preoperative prophylactic interventions should be tailored to the patient's individual risk factors, as identified at the preoperative risk screening. The type of preoperative physical exercise training depends on its rationale. As the rationale is to reduce the hospitalization-induced decline in physical functioning based on a low preoperative lower extremity muscle strength, training should focus on improving lower extremity muscle strength. As lower extremity muscle strength is 
important for practicing transfers, as well as for functional mobility - activities of daily life that are of relevance for patients recovering from major surgery - training should specifically focus on functional task exercises targeting these muscles $[100,101]$.

\section{Participatory}

To be successful in the predictive, preventive, and personalized approach, the individual patient and his/her social support system (e.g., family member, informal caregivers), as primary stakeholders should be involved explicitly. The primary stakeholders should be well-informed about their health care status, treatment-related risks, and advised lifestyle improvements in order to be well-prepared for shared decision-making concerning their treatment with health care professionals [102,103]. This means that health care professionals should recognize and act upon an individual's "health literacy". Health literacy can be defined as "the achievement of a level of knowledge, personal skills, and confidence to take action to improve personal and community health by changing personal lifestyles and living conditions" [104]. Understanding a patient's health literacy is important throughout the perioperative period, so that patients are able to make decisions, which help them to improve or maintain their level of physical functioning. Especially in the preoperative period when patients have a low adaptive capacity to cope with the stress of surgery and hospitalization, a patient's social support system and involved health care professionals should encourage patients to make daily decisions that will enhance their level of physical functioning. Technology and physical therapy can support patients and their social support network in those decisions.

Preoperative physical therapy is not a goal on itself, but a way to support patients in achieving a rapid substantial improvement of physical functioning before hospital admission and surgery. Additionally, it aims to contribute to the patient's ability to actively participate in recovery of physical functioning during and after hospitalization. The physical and social context of physical exercise training is important to increase a patient's adherence, as well as for transferring therapeutic exercises to activities of daily life [105]. This can be achieved by performing functional task exercises. The use of functional task exercises has been reported to be more effective in improving the performance in activities of daily life compared to resistance training, and it is even feasible in highly comprised elderly $[106,107]$. Exercises are more complex and more closely linked to the individual's dynamic environment [106]. To increase physical exercise training adherence, physical therapy should be participatory. This might be achieved by involving the patient's social support network (e.g., partner, relatives), by choosing exercises related to 
activities of daily life that are of relevance, and by jointly objectively monitoring a patient's progression and response to training in the home-based context [100].

\section{From knowledge to practice}

On average it takes 17 years for scientific discoveries to reach clinical practice [108]. Therefore, implementation of research findings into clinical practice has become more important in the scientific inquiry [109]. Health care professionals and researchers should join forces, and focus on the context of patients [110]. To sustainably improve perioperative physical therapy management according to state-of-the art available evidence as described above, research should be more embedded in daily clinical practice [111, 112].

\section{THE EMBEDDED SCIENCE MODEL TO EVALUATE THE TRANSITION IN PERIOPERATIVE CARE}

Many aspects of science are currently under discussion in order to be able to meet and tackle the complex societal challenges. Internationally, there is a debate on how to improve research quality and create more societal impact with scientific research. The Lancet has addressed these issues in five papers and gives recommendations on how to reduce research waste and increase the value of research [113-117]. Furthermore, a working group in the Netherlands, called "Science in Transition", has written a position paper about these matters [118] and the European Commission published a document about Science in Transition 2.0 [119]. To bridge the gap between research findings and clinical practice in health care and to create more value and societal impact [120], research should be more embedded in clinical practice, which facilitates the development, monitoring, and evaluation of interventions in order to improve health care outcomes [112]. The effectiveness of an intervention depends on the context of the patient and setting where care is provided [110]. Consequently, this leads to a more complex intervention, in which process evaluation plays a major role using a mixed methods approach including qualitative and quantitative methodologies [121].

The purpose of this mission-oriented thesis was to provide insight in a transition experiment at the Maastricht University Medical Center, especially in perioperative management of patients that needed major elective abdominal surgery, because of hepatic and pancreatic cancer. The aim of the studies reported in this thesis was to observe, monitor, and evaluate physical fitness, physical functioning, and postoperative outcomes of patients with hepatic and pancreatic cancer during treatment and across the entire transition in perioperative care management, using an embedded science model (see Figure 1.5). 
A.
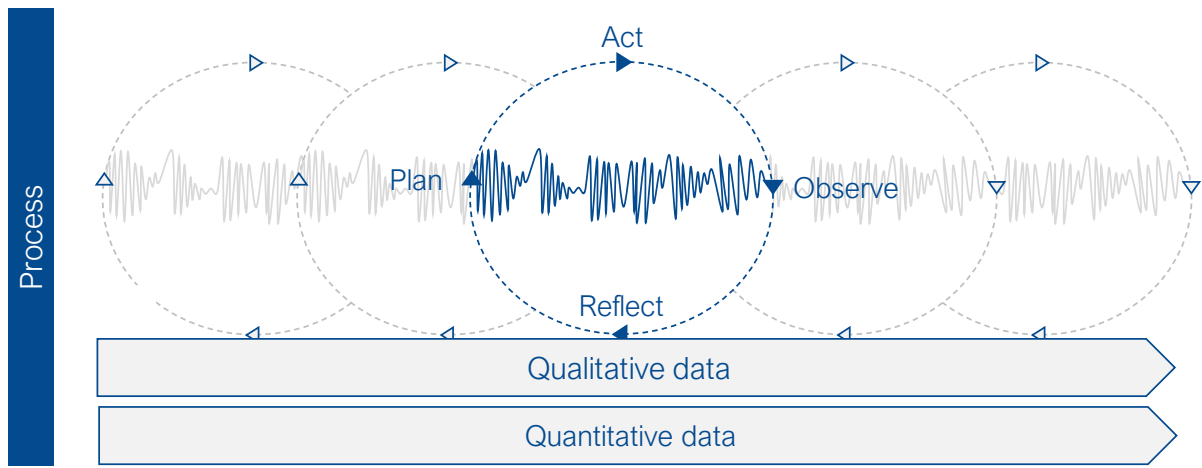

B.
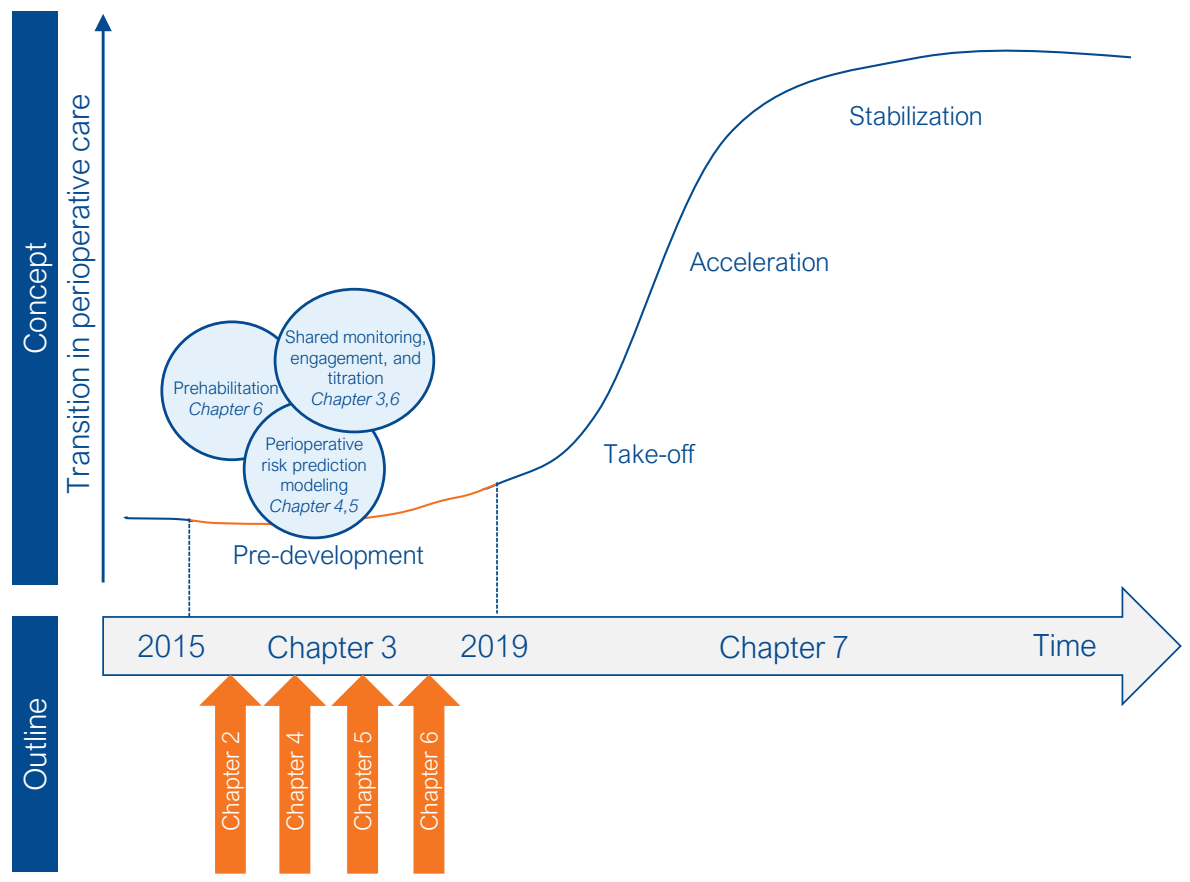

Figure 1.5. The process and concept of the embedded science model combined with the outline of this thesis. Helped help

A. Process: an embedded scientist coordinated the process and analyzed the transition in the perioperative care system by following the plan-act-observe-reflect cycle. Within this process the scientist gathered qualitative and quantitative data. Based on these data, the embedded scientist acted as a change agent in initiating and facilitating the transition experiment.

B. Concept: the concept was the transition in perioperative care, which is characterized as a non-linear process divided in different phases (pre-development, take-off, acceleration, and stabilization phase) with slow and rapid changes. In the pre-development phase, research projects ( $)$ were initiated as part of the mission in perioperative care.

C. Outline: the outline presents the chapters in this thesis, which all fall within the pre-development phase of the transition and describe the process of the pre-development phase (Chapter 3).

This figure and the current thesis provide snapshots about the progress and results anno 2019 in the 'take off' timeframe from 2015 onwards. 
The change-trajectory in time is the transition in perioperative care, from the current care pathway at the start of this mission-oriented research (August 2015) towards a new function-tailored care pathway. The embedded scientist analyzed the system in question (e.g., the ward, the current clinical practice, structure, and culture) and acted as a change agent in initiating and facilitating the transition experiment (e.g., creating opportunities for multidisciplinary preoperative risk stratification, pre- and postoperative physical exercise training, initiating a new perspective on care). Simultaneously, the embedded scientist observed, critically reflected, and analyzed the different actions on the system.

Along this transition in perioperative care for patients undergoing elective HPB surgery at the Maastricht University Medical Center, the following research questions were addressed in this thesis:

- What is the variation between the nowadays advised state-of-the-art perioperative physical therapy management and the routine perioperative physical therapy management in patients undergoing colorectal, liver, or pancreatic surgery in Dutch hospitals (Chapter 2)?

- How to study the transition towards a "P4 health" structure in perioperative physical therapy care management while simultaneously improving pre- and postoperative physical functioning (Chapter 3)

- What is the association between the preoperative level of physical functioning and postoperative outcomes in patients following hepatic or pancreatic surgery (Chapter 4 and Chapter 5)?

- How do preoperative risk assessment and prehabilitation support clinical decision-making in a high-risk patient opting for pancreatic resection (Chapter 6)? 


\section{REFERENCES}

1. Prehabilitation, rehabilitation, and revocation in the Army. Br Med J.1946;1:192-7.

2. Silver JK, Baima J. Cancer prehabilitation: an opportunity to decrease treatment-related morbidity, increase cancer treatment options, and improve physical and psychological health outcomes. Am J Phys Med Rehabil. 2013;92:715-27.

3. Spain J. Prehabilitation. Clin Sports Med. 1985;4:575-85.

4. Topp R, Ditmyer M, King K, Doherty K, Hornyak $J$. The effect of bed rest and potential of prehabilitation on patients in the intensive care unit. AACN Clin Issues. 2002;13:263-76.

5. Kehlet H. Multimodal approach to control postoperative pathophysiology and rehabilitation. Br J Anaesth. 1997;78:606-17.

6. Ljungqvist $\mathrm{O}$, Scott M, Fearon KC. Enhanced recovery after surgery: a review. JAMA Surg. 2017;152:292-8.

7. Fearon $\mathrm{KC}$, Ljungqvist $\mathrm{O}$, Von Meyenfeldt $\mathrm{M}$, Revhaug A, Dejong $\mathrm{CH}$, Lassen $\mathrm{K}$, et al. Enhanced recovery after surgery: a consensus review of clinical care for patients undergoing colonic resection. Clin Nutr. 2005;24:466-77.

8. Hood L, Flores M. A personal view on systems medicine and the emergence of proactive P4 medicine: predictive, preventive, personalized and participatory. N Biotechnol. 2012;29:613-24.

9. Centraal Bureau voor de Statistiek. "Trends in Nederland". 2016:99.

10. Christensen K, Doblhammer G, Rau R, Vaupel JW. Ageing populations: the challenges ahead. Lancet. 2009;374:1196-208.

11. Kehlet $\mathrm{H}$, Wilmore DW. Evidence-based surgical care and the evolution of fast-track surgery. Ann Surg. 2008;248:189-98.

12. Mazzucato M. Mission-oriented research \& innovation in the European Union. Brussels: European Commission. 2018.

13. Mazzucato M. Mission-oriented innovation policies: challenges and opportunities. Industr and Corp Change, 2018;27;803-15.

14. Health-Holland. Knowledge and Innovation Agenda 2020-2023, Top Sector Life Sciences and Health. Schuttelaar \& Partners, Den Haag, 2019.

15. Hoffmann TC, Del Mar CB. Shared decision making: what do clinicians need to know and why should they bother? Med J Aust. 2014;201:5134.
16. Bongers BC, Punt IM, van Meeteren NL. On "Prehabilitation: The emperor's new clothes or a new arena for physical therapists?" Lundberg M, Archer KR, Larsson C, Rydwik E. Phys Ther. 2018;12:127-130. Phys Ther. 2019;99:953-54.

17. Swan M. Health 2050: The realization of personalized medicine through crowdsourcing, the quantified self, and the participatory biocitizen. J Pers Med. 2012;2:93-118.

18. Rotmans J, Kemp R, Van Asselt, M. More evolution than revolution: transition management in public policy. Foresight. 2001;3:15-31.

19. Fleming A, Mason C, Paxton G. Discourses of technology, ageing and participation. Palgrave Commun. 2018;4:54

20. Geels FW. Technological transitions and system innovations: a co-evolutionary and sociotechnical analysis. Cheltenham: Edward Elgar. 2005;318 p.

21. Mazzucato M. A mission-oriented approach to building the entrepreneurial state. 2015.

22. Huber M, Knottnerus JA, Green L, van der Hors $H$, Jadad AR, Kromhout D, et al. How should we define health? BMJ. 2011;343:d4163.

23. Beerda H, Huizenga M. Gemeenschapskracht 2019. Accessed January 2019, https://gemeenschapskracht.nl/wat-wij-doen/.

24. Health-Holland. Year in Review 2018 : Transformation. Schuttelaar \& Partners, Den Hague, 2019.

25. Geels FW. The multi-level perspective on sustainability transitions: Responses to seven criticisms. Environ Innov Soc Trans. 2011;1:2440.

26. Hulzebos EH, van Meeteren NL. Making the elderly fit for surgery. Br J Surg. 2016;103:e1215.

27. Hulzebos EH, van Meeteren NL. Making the elderly fit for surgery. Br J Surg. 2016;103:463.

28. International Agency for Research on Cancer. Population fact sheets cancer 2018. Accessed April 2019,

https://gco.iarc.fr/today/data/factsheets/populatio ns/528-the-netherlands-fact-sheets.pdf.

29. Dutch Institute for Clinical Auditing (DICA) Leiden. DICA Jaarrapportage 2014; DHBA 2014. Accessed April 2019, https://dica.nl/jaarrapportage-2014/dhba.html.

30. McGlynn KA, Tsao L, Hsing AW, Devesa SS, Fraumeni JF. International trends and patterns of primary liver cancer. Int J Cancer. 2001;94:2906. 
31. Dutch Institute for Clinical Auditing (DICA) Leiden. DICA Jaarrapportage 2015; DHBA 2015. Accessed April 2019, https://dica.nl/jaarrapportage-2015/dhba. 2015: Leiden.

32. Dutch Institute for Clinical Auditing (DICA) Leiden. DICA Jaarrapportage 2017. Accessed April 2019, http://dica.nl/jaarrapportage-2017.

33. Lawrence VA, Hazuda HP, Cornell JE, Pederson T, Bradshaw PT, Mulrow CD, Page CP. Functional independence after major abdominal surgery in the elderly. J Am Coll Surg. 2004;199:762-72.

34. Aliberti MJR, Cenzer IS, Smith AK, Lee SJ, Yaffe $\mathrm{K}$, Covinsky KE. Assessing risk for adverse outcomes in older adults: the need to include both physical frailty and cognition. J Am Geriatr Soc. 2019;67:477-83.

35. Covinsky KE, Pierluissi E, Johnston CB. Hospitalization-associated disability: "She was probably able to ambulate, but l'm not sure". JAMA. 2011;306:1782-93.

36. McEwen BS. Stress, adaptation, and disease. Allostasis and allostatic load. Ann N Y Acad Sci. 1998; 840:33-44.

37. McEwen BS. Interacting mediators of allostasis and allostatic load: towards an understanding of resilience in aging. Metabolism. 2003;52:10-6.

38. Ethun CG, Bilen MA, Jani AB, Maithel SK, Ogan $K$, Master VA. Frailty and cancer: Implications for oncology surgery, medical oncology, and radiation oncology. CA Cancer J Clin. 2017;67:362-377.

39. Laviano A, Meguid MM, Inui A, Muscaritoli M, Rossi-Fanelli F. Therapy insight: cancer anorexiacachexia syndrome-when all you can eat is yourself. Nat Clin Pract Oncol. 2005;2:158-65.

40. Fearon K, Strasser F, Anker SD, Bosaeus I, Bruera E, Fainsinger RL, et al. Definition and classification of cancer cachexia: an international consensus. Lancet Oncol. 2011;12:489-95.

41. Laviano A, Meguid MM. Nutritional issues in cancer management. Nutrition. 1996;12:358-71.

42. Dunne RF, Roussel B, Culakova E, Pandya C, Fleming FJ, Hensley B, et al. Characterizing cancer cachexia in the geriatric oncology population. J Geriatr Oncol. 2019;10:415-19.

43. Bortz WM 2nd. A conceptual framework of frailty: a review. J Gerontol A Biol Sci Med Sci. 2002;57:M283-8.

44. Buettner S, Wagner D, Kim Y, Margonis GA, Makary MA, Wilson A, et al. Inclusion of sarcopenia outperforms the modified frailty index in predicting 1-year mortality among 1,326 patients undergoing gastrointestinal surgery for a malignant indication. J Am Coll Surg. 2016;222:397-407.e2.
45. Wagner D, Büttner S, Kim Y, Gani F, Xu L, Margonis GA, et al. Clinical and morphometric parameters of frailty for prediction of mortality following hepatopancreaticobiliary surgery in the elderly. Br J Surg. 2016;103:e83-92.

46. Gemenetzis G, Groot VP, Blair AB, Laheru DA, Zheng L, Narang AK, et al. Survival in ocally advanced pancreatic Cancer After Neoadjuvant Therapy and Surgical Resection. Ann Surg. 2019;270:340-347.

47. Cooper AB, Slack R, Fogelman D, Holmes HM Petzel M, Parker N, et al. Characterization of Anthropometric Changes that Occur During Neoadjuvant Therapy for Potentially Resectable Pancreatic Cancer. Ann Surg Oncol. 2015;22:2416-23.

48. Eriksson S, Nilsson JH, Strandberg Holka P, Eberhard J, Keussen I, et al. The impact of neoadjuvant chemotherapy on skeletal muscle depletion and preoperative sarcopenia in patients with resectable colorectal liver metastases. HPB (Oxford). 2017;19:331-37.

49. Jacquelin-Ravel N, Pichard C. Clinical nutrition, body composition and oncology: a critical literature review of the synergies. Crit Rev Oncol Hematol. 2012;84:37-46.

50. Peng PD, van Vledder MG, Tsai S, de Jong MC, Makary M, et al. Sarcopenia negatively impacts short-term outcomes in patients undergoing hepatic resection for colorectal liver metastasis. HPB (Oxford). 2011;13:439-46.

51. Peng $P$, Hyder $O$, Firoozmand $A$, Kneuertz $P$. Schulick RD, Huang D, et al. Impact of sarcopenia on outcomes following resection of pancreatic adenocarcinoma. J Gastrointest Surg. 2012;16:1478-86.

52. Sandini M, Patino M, Ferrone CR, Alvarez-Pérez CA, Honselmann KC, Paiella S, et al. Association between changes in body composition and neoadjuvant treatment for pancreatic cancer. JAMA Surg. 2018;153:809-15.

53. Hinz A, Krauss O, Hauss JP, Höckel M, Kortmann $\mathrm{RD}$, Stolzenburg JU, et al. Anxiety and depression in cancer patients compared with the general population. Eur J Cancer Care (Engl). 2010;19:522-9.

54. Levett DZH, Grimmett C. Psychological factors, prehabilitation and surgical outcomes: evidence and future directions. Anaesthesia. 2019;74;3642.

55. Bultz BD. Patient Care and Outcomes: Why Cancer Care Should Screen for Distress, the 6(th) Vital Sign. Asia Pac J Oncol Nurs. 2016;3(1):21-4.

56. Wilmore DW. From Cuthbertson to fast-track surgery: 70 years of progress in reducing stress in surgical patients. Ann Surg. 2002;236:643-8. 
57. Burton, D., G. Nicholson, and G. Hall. Endocrine and metabolic response to surgery. Continuing Education in Anaesthesia, Critical Care \& Pain, 2004;5:144-7.

58. Ivanovs I, Mihelsons M, Boka V. Stress response to surgery and possible ways of its correction. Proceedings of the Latvian Academy of Sciences, Section B: Natural, Exact, and Applied Sciences. 2012; 66:225-33.

59. Desborough JP. The stress response to trauma and surgery. Br J Anaesth. 2000;85:109-17.

60. Older $P$, Hall A. Clinical review: how to identify high-risk surgical patients. Crit Care. 2004;8:36972.

61. Older P, Smith R. Experience with the preoperative invasive measurement of haemodynamic, respiratory and renal function in 100 elderly patients scheduled for major abdominal surgery. Anaesth Intensive Care. 1988;16:389-95.

62. Finnerty CC, Mabvuure NT, Ali A, Kozar RA, Herndon DN. The surgically induced stress response. JPEN J Parenter Enteral Nutr. 2013;37:21S-9S.

63. Li L, Messina JL. Acute insulin resistance following injury. Trends Endocrinol Metab. 2009;20:429-35.

64. Ni Choileain N, Redmond HP. Cell response to surgery. Arch Surg. 2006;141:1132-40.

65. Lee L, Tran T, Mayo NE, Carli F, Feldman LS. What does it really mean to "recover" from an operation? Surgery. $2014 ; 155(2): 211-6$.

66. Allvin R, Berg K, Idvall E, Nilsson U. Postoperative recovery: a concept analysis. J Adv Nurs. 2007; $57: 552-8$

67. Moore FD. Getting well: the biology of surgical convalescence. Ann N Y Acad Sci. 1958;73:387400.

68. Carli F, Mayo N. Measuring the outcome of surgical procedures: what are the challenges? $\mathrm{Br}$ J Anaesth. 2001;87:531-3.

69. Sasaki N, Meyer MJ, Eikermann M. Postoperative respiratory muscle dysfunction: pathophysiology and preventive strategies. Anesthesiology. 2013;118:961-78.

70. Ascher RA. The dangers of going to bed. Br Med J. 1947;2:967-8.

71. Brown CJ, Redden DT, Flood KL, Allman RM. The underrecognized epidemic of low mobility during hospitalization of older adults. J Am Geriatr Soc. 2009;57:1660-5.

72. Paddon-Jones D, Sheffield-Moore M, Cree MG, Hewlings SJ, Aarsland A, Wolfe, et al. Atrophy and impaired muscle protein synthesis during prolonged inactivity and stress. J Clin Endocrinol Metab. 2006;91:4836-41.
73. Ikezoe T, Mori N, Nakamura M, Ichihashi N. Atrophy of the lower limbs in elderly women: is it related to walking ability? Eur J Appl Physiol. 2011;111:989-95.

74. Ikezoe T, Mori N, Nakamura M, Ichihashi N. Effects of age and inactivity due to prolonged bed rest on atrophy of trunk muscles. Eur J Appl Physiol. 2012;112:43-8.

75. Kortebein P, Symons TB, Ferrando A, PaddonJones D, Ronsen O, Protas E, et al. Functional impact of 10 days of bed rest in healthy older adults. J Gerontol A Biol Sci Med Sci. 2008;63:1076-81.

76. Ajitsaria P, Eissa SZ, Kerridge RK. Risk Assessment. Curr Anesthesiol Rep. 2018;8:1-8.

77. Jongen AC, van Woerden $\mathrm{V}$, van Vugt JL, de Hoogt PA, de Weerdesteijn EMDW, Tegels JJ, et al. Improving outcome in gastrointestinal and hepatopancreaticobiliary surgical oncology by preoperative risk assessment and optimization of perioperative care. Oncology Critical Care. IntechOpen 2016.

78. Karlsson E, Egenvall M, Farahnak P, Bergenmar $M$, Nygren-Bonnier $M$, et al. Better preoperative physical performance reduces the odds of complication severity and discharge to care facility after abdominal cancer resection in people over the age of 70 - A prospective cohort study. Eur J Surg Oncol. 2018;44:1760-67.

79. Hayashi K, Yokoyama Y, Nakajima H, Nagino M, Inoue T, Nagaya M, et al. Preoperative 6-minute walk distance accurately predicts postoperative complications after operations for hepatopancreato-biliary cancer. Surgery. 2017;161:525-32.

80. Moran J, Wilson F, Guinan E, McCormick P, Hussey J, et al. Role of cardiopulmonary exercise testing as a risk-assessment method in patients undergoing intra-abdominal surgery: a systematic review. Br J Anaesth. 2016;116:177-91.

81. Levett DZH, Jack S, Swart M, Carlisle J, Wilson J, Snowden C, et al.Perioperative Exercise Testing and Training Society (POETTS). Perioperative cardiopulmonary exercise testing (CPET): consensus clinical guidelines on indications, organization, conduct, and physiological interpretation. $\mathrm{Br} \mathrm{J}$ Anaesth. 2018;120:484-500.

82. Older PO, Levett DZH. Cardiopulmonary Exercise Testing and Surgery. Ann Am Thorac Soc. 2017;14:S74-S83.

83. Bongers BC, Berkel AE, Klaase JM, van Meeteren NL. An evaluation of the validity of the pre-operative oxygen uptake efficiency slope as an indicator of cardiorespiratory fitness in elderly patients scheduled for major colorectal surgery. Anaesthesia. 2017;72:1206-16. 
84. Junejo MA, Mason JM, Sheen AJ, Moore J, Foster P, Atkinson D, et al. Cardiopulmonary exercise testing for preoperative risk assessment before hepatic resection. $\mathrm{Br} \mathrm{J}$ Surg. 2012;99:1097-104.

85. Kasivisvanathan R, Abbassi-Ghadi N, McLeod $A D$, Oliver A, Rao Baikady R, Jhanji S, et al. Cardiopulmonary exercise testing for predicting postoperative morbidity in patients undergoing hepatic resection surgery. HPB(Oxford). 2015 Jul;17:637-43.

86. Snowden CP, Prentis J, Jacques B, Anderson H, Manas D, Jones D, et al. Cardiorespiratory fitness predicts mortality and hospital length of stay after major elective surgery in older people. Ann Surg. 2013;257:999-1004.

87. Ulyett S, Wiggans MG, Bowles MJ, Aroori S, Briggs $C D$, Erasmus $P$, et al. Clinical assessment before hepatectomy identifies high-risk patients. J Surg Res. 2015;198:87-92.

88. Junejo MA, Mason JM, Sheen AJ, Bryan A, Moore J, Foster P, et al. Cardiopulmonary exercise testing for preoperative risk assessment before pancreaticoduodenectomy for cancer. Ann Surg Oncol. 2014;21:1929-36

89. Chandrabalan VV, McMillan DC, Carter R, Kinsella J, McKay CJ, Carter CR, et al. Preoperative cardiopulmonary exercise testing predicts adverse post-operative events and nonprogression to adjuvant therapy after major pancreatic surgery. HPB (Oxford). 2013;15:899907.

90. Kumar R, Garcea G. Cardiopulmonary exercise testing in hepato-biliary \& pancreas cancer surgery - A systematic review: are we any further than walking up a flight of stairs? Int J Surg. 2018;52:201-7.

91. Moran J, Wilson F, Guinan E, McCormick P, Hussey J, Moriarty J. The preoperative use of field tests of exercise tolerance to predict postoperative outcome in intra-abdominal surgery: a systematic review. J Clin Anesth. 2016;35:446-55.

92. Huisman MG, van Leeuwen BL, Ugolini G, Montroni I, Spiliotis J, Stabilini C, et al. "Timed Up \& Go": a screening tool for predicting 30-day morbidity in onco-geriatric surgical patients? A multicenter cohort study. PLoS One. 2014;9:e86863.

93. Reddy S, Contreras CM, Singletary B, Bradford TM, Waldrop MG, Mims AH, et al. Timed stair climbing is the single strongest predictor of perioperative complications in patients undergoing abdominal surgery. J Am Coll Surg. 2016;222:559-66.

94. Cederholm T, Barazzoni R, Austin P, Ballmer P, Biolo G, Bischoff SC, et al. ESPEN guidelines on definitions and terminology of clinical nutrition. Clin Nutr. 2017;36:49-64.
95. Griffin O, Conlon KC. Sarcopenia - a new frontier in the management care of patients with borderline resectable pancreatic cancer. JAMA Surg. 2018;153:816.

96. Barberan-Garcia A, Ubré M, Roca J, Lacy AM, Burgos F, Risco R, et al. Personalised prehabilitation in high-risk patients undergoing elective major abdominal surgery: a randomized blinded controlled trial. Ann Surg. 2018;267:506.

97. Scheede-Bergdahl C, Minnella EM, Carli F. Multimodal prehabilitation: addressing the why, when, what, how, who and where next? Anaesthesia. 2019;74:20-6.

98. Nakajima H, Yokoyama $\mathrm{Y}$, Inoue T, Nagaya M, Mizuno Y, Kadono I, et al. Clinical benefit of preoperative exercise and nutritional therapy for patients undergoing hepato-pancreato-biliary surgeries for malignancy. Ann Surg Oncol. 2019;26:264-72.

99. Dunne DF, Jack S, Jones RP, Jones L, Lythgoe DT, Malik HZ, et al. Randomized clinical trial of prehabilitation before planned liver resection. $\mathrm{Br} \mathrm{J}$ Surg. 2016;103:504-12.

100. Dronkers J, Witteman B, van Meeteren N. Surgery and functional mobility: doing the right thing at the right time. Tech Coloproctol. 2016;20:339-41.

101. de Vreede PL, Samson MM, van Meeteren NL, van der Bom JG, Duursma SA, Verhaar HJ. Functional tasks exercise versus resistance exercise to improve daily function in older women: a feasibility study. Arch Phys Med Rehabil. 2004;85:1952-61.

102. Hood L. Systems biology and P4 medicine: past, present, and future. Rambam Maimonides Med J. 2013;4:e0012.

103. Sturgess J, Clapp JT, Fleisher LA. Shared decision-making in peri-operative medicine: a narrative review. Anaesthesia. 2019;74:13-9.

104. Nutbeam D. Health promotion glossary. Health promotion international. 1998;13:349-64.

105. Schmidt RA, Lee TD. Motor learning and performance: from principles to application. Human Kinetics, 2018.

106. de Vreede PL, Samson MM, van Meeteren NL, Duursma SA, Verhaar HJ. Functional-task exercise versus resistance strength exercise to improve daily function in older women: a randomized, controlled trial. J Am Geriatr Soc. 2005;53:2-10.

107. Siemonsma PC, Blom JW, Hofstetter H, van Hespen ATH, Gussekloo J, Drewes YM, et al. The effectiveness of functional task exercise and physical therapy as prevention of functional decline in community dwelling older people with complex health problems. BMC Geriatr. 2018;18:164. 
108. Morris ZS, Wooding S, Grant J. The answer is 17 years, what is the question: understanding time lags in translational research. J R Soc Med. 201;104(12):510-20.

109. Rapport F, Clay-Williams R, Churruca K, Shih P, Hogden A, Braithwaite J. The struggle of translating science into action: Foundational concepts of implementation science. J Eval Clin Pract. 2018;24:117-26.

110. Raad van Volksgezondheid en Samenleving. No evidence without context. About the illusion of evidence-based practice in healthcare. 2017. Accessed April 2019, https://www.raadrvs.nl/documenten/publications/ 2017/6/19/no-evidence-without-context.-aboutthe-illusion-of-evidence\%E2\%80\%90basedpractice-in-healthcare.

111. Grol R, Grimshaw J. From best evidence to best practice: effective implementation of change in patients' care. Lancet. 2003;362:1225-30.

112. Craig P, Cooper C, Gunnell D, Haw S, Lawson K, Macintyre S, et al. Using natural experiments to evaluate population health interventions: new Medical Research Council guidance. J Epidemiol Community Health. 2012;66:1182-6.

113. Chalmers I, Bracken MB, Djulbegovic B, Garattini S, Grant J, Gülmezoglu AM, et al. How to increase value and reduce waste when research priorities are set. Lancet. 2014;383:156-65.

114. Ioannidis JP, Greenland S, Hlatky MA, Khoury MJ, Macleod MR, Moher D, et al. Increasing value and reducing waste in research design, conduct, and analysis. Lancet. 2014;383:166-75.
115. Al-Shahi Salman R, Beller E, Kagan J, Hemminki E, Phillips RS, Savulescu J, et al. Increasing value and reducing waste in biomedical research regulation and management. Lancet. 2014;383:176-85.

116. Chan AW, Song F, Vickers A, Jefferson T, Dickersin K, Gøtzsche PC, et al. Increasing value and reducing waste: addressing inaccessible research. Lancet. 2014;383:257-66.

117. Glasziou P, Altman DG, Bossuyt P, Boutron I, Clarke M, Julious S, et al. Reducing waste from incomplete or unusable reports of biomedical research. Lancet. 2014;383:267-76.

118. Dijstelbloem H, Huisman F, Miedema F, Mijnhardt $W$. Waarom de wetenschap niet werkt zoals het moet en wat daar aan te doen is. in Position paper. 2013, Science in Transition. p. 31.

119. European Union Commission. Validation of the results of the public consultation on Science 2.0: Science in Transition. Retrieved July 10, 2015.

120. Glasgow RE, Lichtenstein E, Marcus AC. Why don't we see more translation of health promotion research to practice? Rethinking the efficacy-toeffectiveness transition. Am J Public Health. 2003;93:1261-7.

121. Moore GF, Audrey S, Barker M, Bond L, Bonell C, Hardeman W, et al. Process evaluation of complex interventions: Medical Research Council guidance. BMJ. 2015;350:h1258.

\section{NOTES}


Er is een grote discrepantie tussen de dagelijkse klinische praktijk en evidence-based aanbevelingen betreffende pre-en postoperatieve ziekenhuisfysiotherapie voor patiënten die grote abdominale chirurgie ondergaan. 


\section{Chapter 2}

Variation in reported preoperative and postoperative physical therapy management for patients opting for major elective abdominal surgery

Christel A. van Beijsterveld ${ }^{*}$ | Aniek F. Heldens* | Bart C. Bongers |

Nico L. van Meeteren

* Shared first authorship 


\section{ABSTRACT}

\section{Background}

Evidence about the role of physical therapy in perioperative care pathways to improve postoperative outcomes is growing. However, it is unclear whether research findings have been translated in daily practice.

\section{Method}

Hospital physical therapists were asked to complete an online survey about pre- and postoperative physical therapy at their hospital. To explore variability of perioperative physical therapy management between hospitals, frequency variables were clustered to determine the level of uniformity. Latent class analysis (LCA) was performed to identify clusters of hospitals with certain homogenous characteristics on a 19-item dichotomous scale.

\section{Results}

Of 82 eligible Dutch hospitals, 65 filled out the survey (79.3\%). Preoperative physical therapy was performed in 34 hospitals (53.9\%). Postoperative physical therapy was performed in all respondents, focusing mainly on regaining independent physical functioning. LCA identified a three-class model. Hospitals in class I and II were more likely to provide preoperative physical therapy interventions compared to class III.

\section{Conclusion}

There is a wide degree of variability between hospitals regarding pre- and postoperative clinical physical therapy practice for patients opting for major abdominal surgery. Three different classes of daily practice were identified. Further translation of key research findings into clinical physical therapy practice is advised, especially for hospitals in which the physical therapist is not involved preoperatively. Moreover, improving uniformity by developing up-to-date clinical guidelines is recommended.ground 


\section{INTRODUCTION}

According to the World Health Organization, the number of people aged 65 and older is expected to increase to 1.5 billion in 2050, representing $16.0 \%$ of the world's population [1]. With the aging population, the number of elderly requiring surgical procedures is increasing. Elderly patients may experience difficulties when recovering from surgery due to diminished physiological reserves, frailty, and comorbidities [2-4]. In the last decades, beneficial improvements have therefore been made in perioperative care management. Developments in analgesic approaches and surgical techniques, as well as "enhanced recovery after surgery" (ERAS) and "fast track" programs resulted in a reduced length of hospital stay and a reduction in overall complications [5].

However, the stress response to surgery and hospitalization comprises metabolic and physiological changes and results in an increase in allostatic load [6, 7]. A vulnerable patient with comorbid conditions and reduced physiological reserve opting for elective major surgery may have less capacity to adapt to the increased allostatic load that may lead to an imbalance in autonomic, endocrine, metabolic, and immune function, resulting in additional clinical challenges leading to a delayed recovery of physical functioning [8], or even a permanent loss of physical functioning.

Hereto, a change in the continuum of care is advocated with complementary prevention and care interventions to assist the vulnerable patient in managing their perioperative course [9-11]. This implies a changing role for the physical therapist in acute care settings concerning patient care and professionalism, as already mentioned by Lopopolo et al. in 1999 [12]. Improvements in perioperative care should best include innovative physical therapy interventions to prevent a complicated peri- and postoperative course, as well as to enhance a rapid return to adequate performance of activities of daily life that is essential to preserve independent physical functioning and perceived quality of life. Innovative pre- and postoperative physical therapy interventions should lead towards realization of predictive, preventive, personalized, and participatory (P4) perioperative health [13]. The latter means including preoperative risk stratification using performance-based tests, preoperative advice and recommendations about the importance of physical activity and physical fitness, preoperative exercise training for high-risk patients, and early mobilization and functional physical exercise training postoperatively, as recommended in several guidelines $[9,10,14-17]$. High-quality research demonstrates that preoperative physical therapy interventions for high-risk patients undergoing cardiac, abdominal, and major joint replacement surgery are feasible and effective [18-22]. 
It remains unclear to what degree these research findings already have been translated and implemented in the real-life context of health care systems, especially in the routines of physical therapy services. Consequently, it is necessary to establish an understanding of the current clinical practice in order to compare the differences between clinical research outcomes and clinical practice, as well as to anticipate on what should be undertaken to change clinical practice and education for physical therapy students according to the latest evidence. Nationwide surveys have been performed to explore the clinical physical therapy management in different fields to examine practice variability with respect to known evidence and guidelines [23-27]. Benefits of such an approach were shown previously by Peter et al. [27] with improvements of guideline recommendations for post-acute physical therapy practice after major joint replacement. To provide optimal physical therapy care for patients opting for major abdominal surgery this approach might be beneficial as well. Therefore, the aims of the present study were two-fold: 1) to describe the overall reported content and between-hospital variability of perioperative physical therapy management in patients who opt for elective major abdominal surgery for colorectal, hepatic, or pancreatic cancer in the Netherlands, and 2) compare the nowadays-advised stateof-the-art physical therapy with the self-reported daily clinical physical therapy management. With the state-of-the-art physical therapy management, we refer to the current highest level of scientific evidence available in perioperative physical therapy management.

\section{METHODS}

\section{Design}

In this cross-sectional survey study, all heads of the departments of physical therapy who were registered with the Dutch Association for Physical therapy in Hospitals (NVZF) and the Association of Intramural Physical therapy Managers (VLF) were contacted in November 2016 by e-mail. They were informed about the purpose of the study and the content of the survey. Furthermore, they were asked to forward a participant information letter with the survey to the physical therapist at their department with the most expertise in working with patients undergoing elective major abdominal surgery for colorectal, hepatic, or pancreatic cancer. An e-mail reminder was sent each month for three months to the heads of the departments of physical therapy of which no response was yet obtained. Additionally, two short news items were published in the monthly newsletters of both the NVZF and VLF that described the study and explained how to participate. One news item was published at the beginning of the study and one after three months. Hospitals that not yet filled out the survey after three months were contacted by phone. 


\section{Ethics}

The participant information letter clearly stated that only coded survey data will be used for publication. Because of the voluntary nature of the survey without patient involvement, the study does not meet the criteria for the Dutch medical research involving human subjects act (Wmo). Therefore, assessment by a medical ethical review committee was not necessary. None of the respondents objected and a full response on the survey was interpreted as informed consent.

\section{Participants}

Physical therapists employed in an acute-hospital care setting in the Netherlands were inquired by a survey about the preoperative and postoperative physical therapy management at their hospital in patients undergoing elective colorectal, hepatic, or pancreatic resection. Hospitals not performing these types of surgery were excluded.

\section{Survey}

The survey consisted of 41 mixed questions (six open and 35 multiple choice) in the following four domains: 1) demographic data (five questions), 2) preoperative diagnostics and treatment data (twelve questions), 3) postoperative diagnostics and treatment data (seventeen questions), and 4) discharge and readmission data (seven questions). The survey was mainly based on a survey study with a similar research aim [26]. The current survey was larded and shaped with concepts of the framework of Hulzebos \& van Meeteren $[9,10]$ and a literature review to be able to inquire whether hospitals provide the nowadays-advised state-of-the-art pre- and postoperative physical therapy management in patients scheduled for elective major abdominal surgery. Additionally, the survey included six questions concerning the context of the postoperative physical therapy treatment (two items) and the availability and usefulness of a readmission protocol (four items). The final version of the survey was peer-reviewed by three (hospital) physical therapists with experience and knowledge about the perioperative care pathway at the Maastricht UMC+. The survey was piloted in a semi-structured process using written and verbal feedback of the assessors. The survey was evaluated on the number and type of questions, relevance of each question, wording, and whether the survey questions captured the total perioperative care pathway. The survey is available in appendix 2.1 (translated into English, not an official cross-cultural adaptation). The survey was developed and administered using Qualtrics electronic survey software (www.qualtrics.com) provided by Maastricht University, Maastricht, the Netherlands. This manuscript is reported according to the STROBE guideline for cross-sectional studies. 


\section{Data analysis}

Two researchers ( $\mathrm{CB}$ and $\mathrm{AH}$ ) categorized the answers of the open questions. When differences between assessors were found, a third researcher (BB) was involved to obtain consensus. The Statistical Package for the Social Sciences for Windows (version 23.0; IBM, SPSS inc., Chicago, IL, USA) was used for the descriptive data analysis, which was used to describe the study population and responses to the survey. To explore the variability of perioperative physical therapy management between hospitals, frequency variables were clustered to determine the level of uniformity, presented as the percentage of respondents choosing the same answer. The categories of uniformity were classified as follows: 1) no uniformity (<60.0\%), 2) low uniformity (61.0\%-70.0\%), 3) moderate uniformity (71.0\%-80.0\%), 4) strong uniformity (81.0\%-90.0\%), and 5$)$ very strong uniformity $(91.0 \%-100.0 \%)$.

To compare the nowadays-advised state-of-the-art physical therapy with the self-reported daily clinical physical therapy practice, we extracted several items from each subdomain of the survey that are closely related to the described conceptual hypothesis in the literature for perioperative physical therapy management. These items were independently extracted from the survey by three individual assessors ( $\mathrm{CB}, \mathrm{AH}$, and $\mathrm{BB}$ ) and resulted in a 19-item dichotomous scale containing seven items on preoperative diagnostics and treatment, eight items addressing postoperative diagnostics and treatments, and four items concerning discharge and readmission (items listed in Table 2.4). Based on the reported clinical physical therapy management, each hospital was subsequently scored on the 19-item dichotomous scale.

Exploratory latent class analysis (LCA) was used to identify and classify clusters of hospitals with certain homogenous characteristics on the 19-items dichotomous scale. Selection of the optimal number of classes was based on several goodness-of-fit parameters. The following statistical fitindices were used in our study: the Akaike information criterion (AIC) and the Bayesian information criteria (BIC). Lower AIC and BIC values indicate a better fit. Furthermore, selection of the classes were based on their substantive meaningfulness (the classes should be distinct and meaningful for the clinical expert). LCA was performed with the open source statistical package R (version 2.14.2, R Foundation for Statistical Computing, Vienna, Austria) using the package poLCA [28] version 1.4. In addition, an index (frequency distribution) score was calculated based on the given response by the hospitals on all items at the 19-item dichotomous scale. These scores represent the distance between the nowadays-advised state-of-the-art physical therapy and the self-reported daily clinical physical therapy management. Respondents 
with scores above $75.0 \%$ were classified as "progressive", respondents with scores between $50.0 \%$ and $75.0 \%$ were classified as "moderately progressive", and respondents with scores lower than $50.0 \%$ were classified as "conservative".

\section{RESULTS}

Flow of participants through the study

Of the 103 Dutch hospitals, 21 hospitals (20.4\%) were excluded on beforehand, as they did not perform colorectal, hepatic, or pancreatic resection. The remaining 82 hospitals (82/103, 79.6\%) were contacted for participation in the survey. After an inclusion period of three months, 65 of these 82 eligible hospitals responded to our survey, resulting in a response rate of $79.3 \%$. Two responding hospitals $(2 / 65,3.0 \%)$ were excluded from data analysis, since they did not correspond with the inclusion criteria performing elective colorectal, hepatic, or pancreatic resection in their hospital. Hence, a total of 63 surveys (63/82, 76.8\%) were included for data analysis. Demographic and descriptive data concerning the reported pre- and postoperative clinical physical therapy management from the responding hospitals are provided in Table 2.1 and 2.2. The flowchart of the study is showed in Figure 2.1.

Table 2.1. Demographic data of the responding hospitals $(n=63)$.

\begin{tabular}{|lll|}
\hline Type of hospital & $\mathrm{n}$ & $(\%)$ \\
\hline Academic hospital & 8 & $(12.7)$ \\
\hline General hospital & 52 & $(82.5)$ \\
\hline Other & 3 & $(4.8)$ \\
\hline Education level of hospital physical therapist & Mean $(\mathrm{SD}) \%$ \\
\hline Bachelor of applied Science degree & 76.3 & $(7.4)$ \\
\hline Clinical specialization in physical therapy & 16.2 & $(3.3)$ \\
\hline Master of Science degree & 6.6 & $(2.1)$ \\
\hline Doctor of Philosophy degree & 1.0 & $(0.0)$ \\
\hline Standardized care pathway for major abdominal surgery & $\mathrm{n}$ & $(\%)$ \\
\hline Yes & 43 & $(63.3)$ \\
\hline Physical therapist involved in the development & 31 & $(72.1)$ \\
\hline No & 20 & $(31.7)$ \\
\hline Type of standardized care pathway (n=43) ${ }^{\text {a }}$ & $\mathrm{n}$ & $(\%)$ \\
\hline Enhanced recovery after surgery & 27 & $(62.8)$ \\
\hline Fast-track & 10 & $(23.3)$ \\
\hline Other & 8 & $(18.6)$ \\
\hline Protocol-guided physical therapy & $\mathrm{n}$ & $(\%)$ \\
\hline Yes & 49 & $(77.8)$ \\
\hline No & 14 & $(22.2)$ \\
\hline a: Multiple answers possible. & & \\
\hline
\end{tabular}


Table 2.2. Reported pre- and postoperative clinical physical therapy management by physical therapists in patients who underwent elective colorectal, hepatic, or pancreatic resection.

\section{Preoperative physical therapy management}

Preoperative physical therapy intervention (e.g., patient education, assessment of physical fitness and prehabilitation) ${ }^{a}$

\begin{tabular}{lll} 
Yes a & 34 & $(54.0)$ \\
No & 29 & $(46.0)$ \\
Preoperative physical therapy intervention $^{b}$ & $n$ & $(\%)$ \\
$\quad$ Usual care & 15 & $(44.1)$ \\
\hline Only after referral (specialist physician, case manager, nurse practitioner) & 19 & $(55.9)$ \\
Content preoperative physical therapy intervention $(n=34)^{c}$ & $n$ & $(\%)$ \\
\hline Patient education & 30 & $(88.2)$ \\
Assessment of physical fitness level & 20 & $(58.8)$ \\
Physical training (prehabilitation) & 11 & $(32.4)$ \\
\hline Postoperative physical therapy management & $n$ & $(\%)$
\end{tabular}

Postoperative physical therapy as part of usual care
Yes

Yes 43

No 20

Referrer for postoperative physical therapy ${ }^{\circ}$

\begin{tabular}{lll} 
Ward physician & 46 & $(73.0)$ \\
\hline Surgeon & 42 & $(66.7)$ \\
\hline Nurse & 35 & $(55.6)$ \\
\hline Physical therapist & 26 & $(41.3)$ \\
Nurse practitioner & 21 & $(33.3)$ \\
\hline Other & 4 & $(6.3)$
\end{tabular}

Postoperative physical therapy treatment frequency

Once a day

$47 \quad(74.6)$

Once or twice a day

Twice a day

5

Other (depends on individual patient)

2

Postoperative physical therapy treatment session duration (minutes)

$10 \quad 9$

$15-23$

$20 \quad 30 \quad(47.6)$

$30 \quad 1$

Postoperative physical therapy treatment session during weekends

$\begin{array}{lll}\text { Yes } & 60 & \text { (95.2) } \\ \text { No } & 3 & (4.8)\end{array}$

a. The number of hospitals in which a preoperative intervention to the physical was part of the routine perioperative care pathway $(n=34,34 / 63=54.0 \%)$.

b: The distribution of "yes" and "no" about the preoperative referral to the physical therapy department in the 34 hospitals in which a preoperative physical therapy intervention was part of the care pathway.

c: Multiple answers possible. 
Table 2.2. Continued.

\section{Discharge and readmission}

Decision for hospital discharge

Shared decision-making 26

Surgeon

$22(34.9)$

Ward physician

$15 \quad(23.8)$

Specific hospital discharge criteria available

$\begin{array}{lll}\text { Yes } & 42 & (66.7) \\ \text { No } & 21 & (33.3)\end{array}$

Specific readmission protocol available

$\begin{array}{lll}\text { Yes } & 0 & (0.0) \\ \text { No } & 24 & (38.1) \\ \text { Unknown } & 39 & (61.9)\end{array}$

Opinion concerning the usefulness of a readmission protocol

\begin{tabular}{lll} 
Useful & 3 & $(4.8)$ \\
\hline Neutral & 29 & $(46.0)$ \\
Not useful & 31 & $(49.2)$
\end{tabular}

Content and variability in the reported physical therapy management

Out of 63 respondents, 34 (53.9\%) reported that patients were seen preoperatively by a physical therapist, of which most patients were seen once $(31 / 34,91.1 \%)$. The content of the preoperative intervention consisted of patient education (30/34, 88.2\%), assessment of physical fitness (20/34, 58.8\%), and/or prehabilitation (11/34, 32.3\%). There was a strong uniformity between hospitals (30/34, 88.2\%) reporting about the elements of education for patients (see Figure 2.2, graph la). A total of 20 respondents (20/34, 58.8\%) reported they measured the patient's preoperative physical fitness level prior to surgery. Strong uniformity concerning the importance of the assessment of daily physical activity level (18/20, 90.0\%) and functional mobility (17/20, 85.0\%) was observed. There was no uniformity concerning other domains of the preoperative physical fitness assessment (see Figure 2.2, graph lb). No uniformity was seen for the type of preoperative physical performance tests and the type of preoperative questionnaires (see Figure 2.2, graph Ic). Regarding the type of exercise prehabilitation, moderate-to-low uniformity was observed for inspiratory muscle strength training (8/11, 72.7\%), peripheral muscle strength training $(7 / 11,63.6 \%)$, and cardiorespiratory exercise training $(7 / 11,63.6 \%)$. 


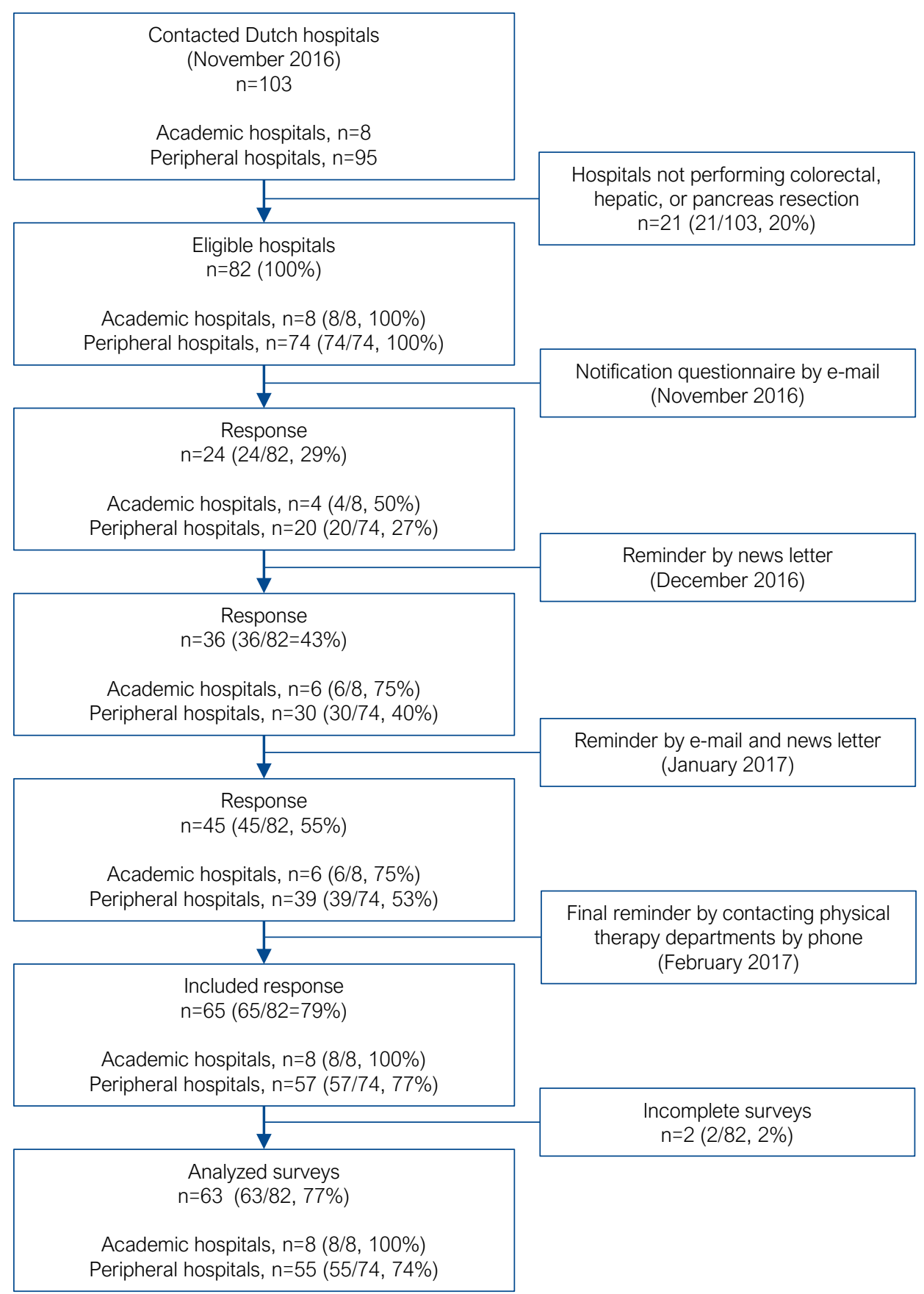

Figure 2.1. Flowchart of the study. 
For functional exercise training $(4 / 11,36.3 \%)$ and breathing exercises $(2 / 11,18.2 \%)$, no uniformity was observed (see 2.2, graph Id). The majority of the respondents (10/11, 90.9\%) reported that not all patients were eligible for exercise prehabilitation, in which the decision for preoperative physical exercise training was based on the risk profile of the patient $(5 / 10,50.0 \%)$, the request of the surgeon $(2 / 10,20.0 \%)$, or other reasons, like the age of the patients, number of comorbidities, or need for inspiratory muscle training (3/10, 30.0\%).

la. Elements of education for patients ( $n=30,30 / 34,88 \%)$

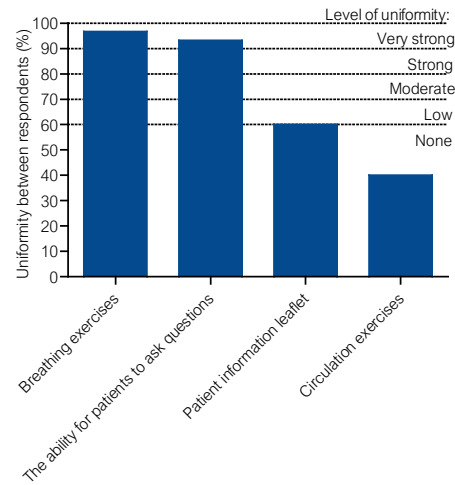

Ic. Type of performance tests to assess physical fitness used by physical therapists ( $n=16,16 / 34,47 \%)$
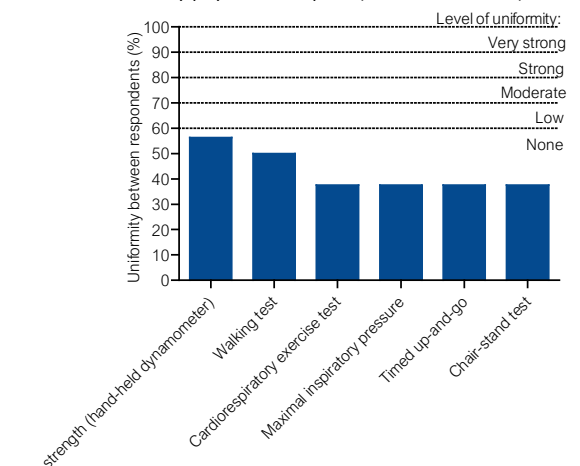

Ib. Domains of physical fitness assessed by physical therapists $(n=20,20 / 34,59 \%)$

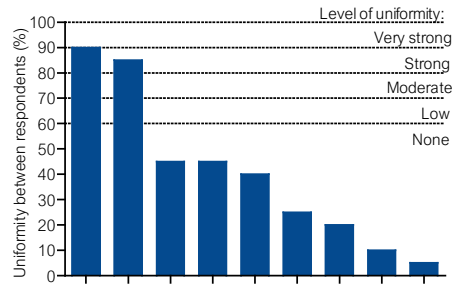

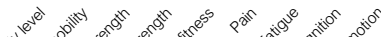

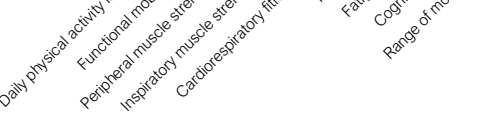

Id. Type of exercise prehabiliation ( $n=11,11 / 34,32 \%)$

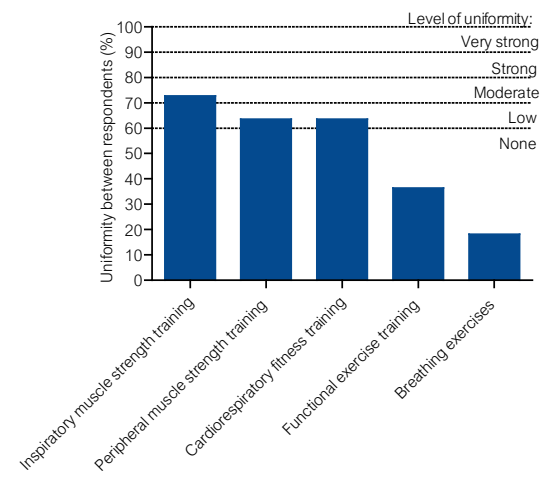

Figure 2.2. Reported preoperative physical therapy interventions $(n=34)$ performed by physical therapists in patients following elective colorectal, hepatic, or pancreatic resection, including elements of education (la, $n=30)$, domains of physical fitness assessment used by physical therapists $(\mathrm{lb}, \mathrm{n}=20)$, type of performance tests to assess physical fitness used by physical therapists (lc, $n=16$ ), and components of exercise prehabilitation (Id, $n=11$ ). 
Postoperative physical therapy practice of which (very) strong uniformity was observed between the respondents $(n=63)$ were breathing exercises $(62 / 63,98.4 \%)$, practicing transfers $(62 / 63$, 98.4\%), patient education (61/63, 96.8\%), stair climbing (56/63, 88.8\%), and walking exercises (55/63, 87.3\%) (see Figure 2.3, graph la). Low or no uniformity was observed for postoperative physical performance tests to monitor and evaluate a patient's recovery of physical functioning (see Figure 2.3, graph Ib).

\section{la. Content of postoperative physical therapy treatment $(n=63,63 / 63,100 \%)$}
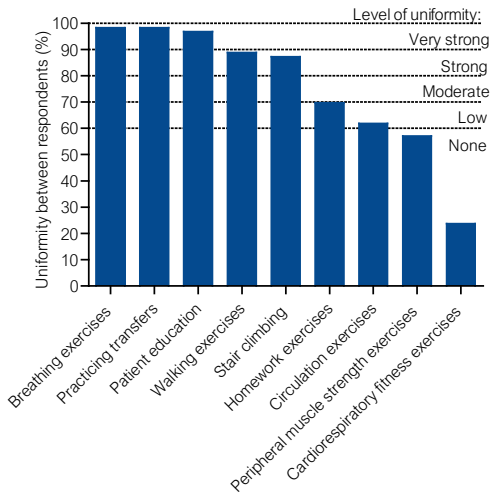

Ib. Postoperative performance tests for monitoring patients' outcome $(n=25,25 / 63,40 \%)$
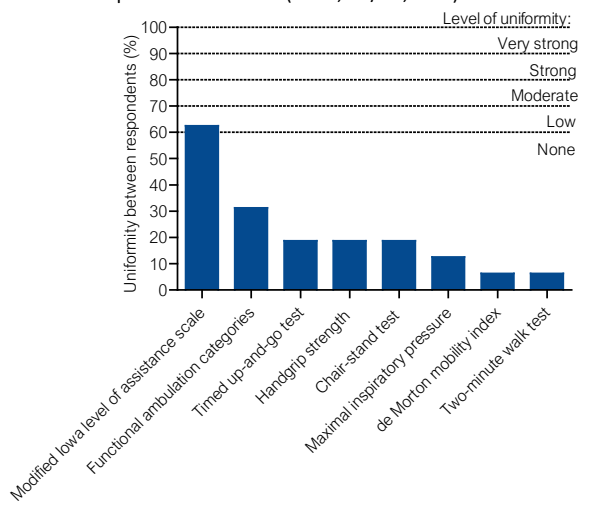

Figure 2.3. Reported postoperative physical therapy interventions $(n=63)$ performed by physical therapists in patients following elective colorectal, hepatic, or pancreatic resection including, content of postoperative physical therapy treatment $(\mathrm{la}, \mathrm{n}=63)$ and postoperative performance tests for monitoring patient's outcome ( $\mathrm{lb}, \mathrm{n}=25)$. 
Comparison between reported physical therapy management and advised state-of-the-art physical therapy

LCA identified a three-class model based on the AIC (988.52), BIC (1102.10) and substantive relevance. The fit-indices from the LCA are reported in Table 2.3. LCA assigned each hospital to one of the three classes (posterior probability) and calculated the conditional probabilities (value between 0 and 1) of each item for each class (see Table 2.4). For the items addressing preoperative physical therapy, respondents in class I and II were more likely to respond that they provide preoperative physical therapy for patients than respondents in class III. There was a 94.0\% chance that a hospital in latent class I provided a preoperative assessment of physical fitness and a 50.0\% chance that this respondent provided prehabilitation, whereas these probabilities for respondents in class II were $28.0 \%$ (providing a preoperative assessment) and $17.0 \%$ (providing prehabilitation), respectively. For postoperative physical therapy management, no major differences were seen in the conditional probabilities between the three classes. Calculating the index scores for each hospital, meaning the distance between the reported physical therapy practice and advised state-of-the-art physical therapy, the same patterns as the LCA classes are observed in the pre- and postoperative physical therapy. Figures 2.4, 2.5, 2.6, and 2.7 show the index scores for each hospital. These figures also demonstrate average response profiles for each group on the 19-item dichotomous scale.

\begin{tabular}{|c|c|c|c|c|c|}
\hline Nclass & Log-likelihood & $\mathrm{BIC}$ & $\mathrm{AlC}$ & Df & Npar \\
\hline 1 & -565.7281 & 1201.889 & 1165.4562 & 46 & 17 \\
\hline 2 & -474.4141 & 1093.838 & 1018.8282 & 28 & 35 \\
\hline 3 & -441.2581 & 1102.102 & 988.5162 & 10 & 53 \\
\hline 4 & -430.8282 & 1155.819 & 1003.6563 & -8 & 71 \\
\hline
\end{tabular}

Abbreviations: $\mathrm{AIC}=$ Akaike's information criterion; $\mathrm{BIC}=$ Schwarz's Bayesian information criterion; $\mathrm{Df}=$ degrees of freedom; Nclass=number of classes; Npar=number of parameters. 
Table 2.4. The descriptive statistics on each item on the 19-item dichotomous scale and the class response probability: the probabilities of reporting "yes" on each item of each class.

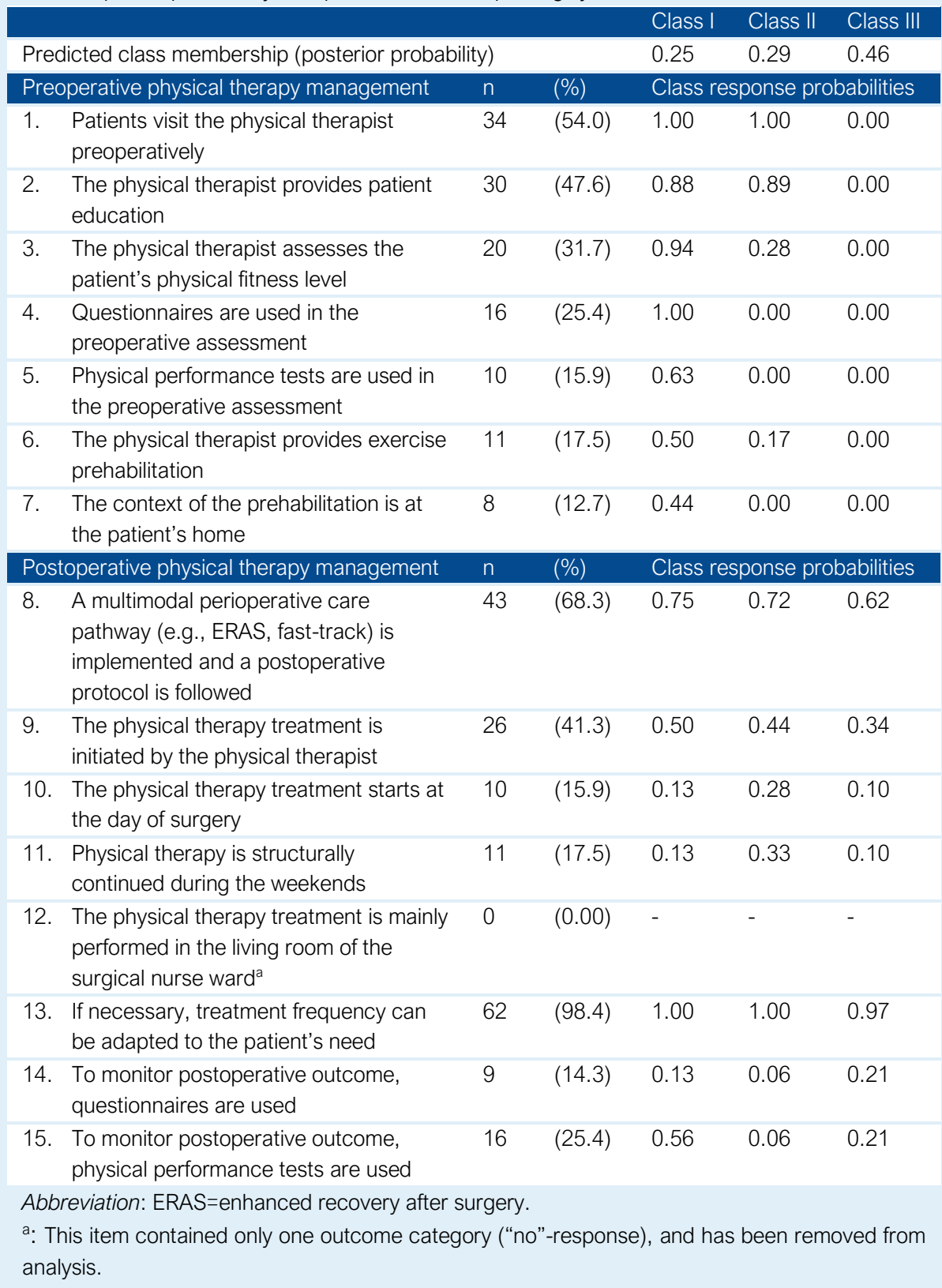


Table 2.4. Continued.

\begin{tabular}{|c|c|c|c|c|c|}
\hline Discharge and readmission & $\mathrm{n}$ & $(\%)$ & \multicolumn{3}{|c|}{ Class response probabilities } \\
\hline $\begin{array}{l}\text { Discharge is performed by shared decision- } \\
\text { making }\end{array}$ & 26 & (41.3) & 0.56 & 0.56 & 0.24 \\
\hline Discharge criteria are available & 42 & $(66.7)$ & 0.88 & 0.72 & 0.52 \\
\hline A readmission protocol is available ${ }^{a}$ & 0 & $(0.0)$ & - & - & - \\
\hline $\begin{array}{l}\text { In case of a readmission, the physical therapist } \\
\text { is timely consulted }\end{array}$ & 48 & $(76.2)$ & 0.75 & 0.67 & 0.82 \\
\hline
\end{tabular}
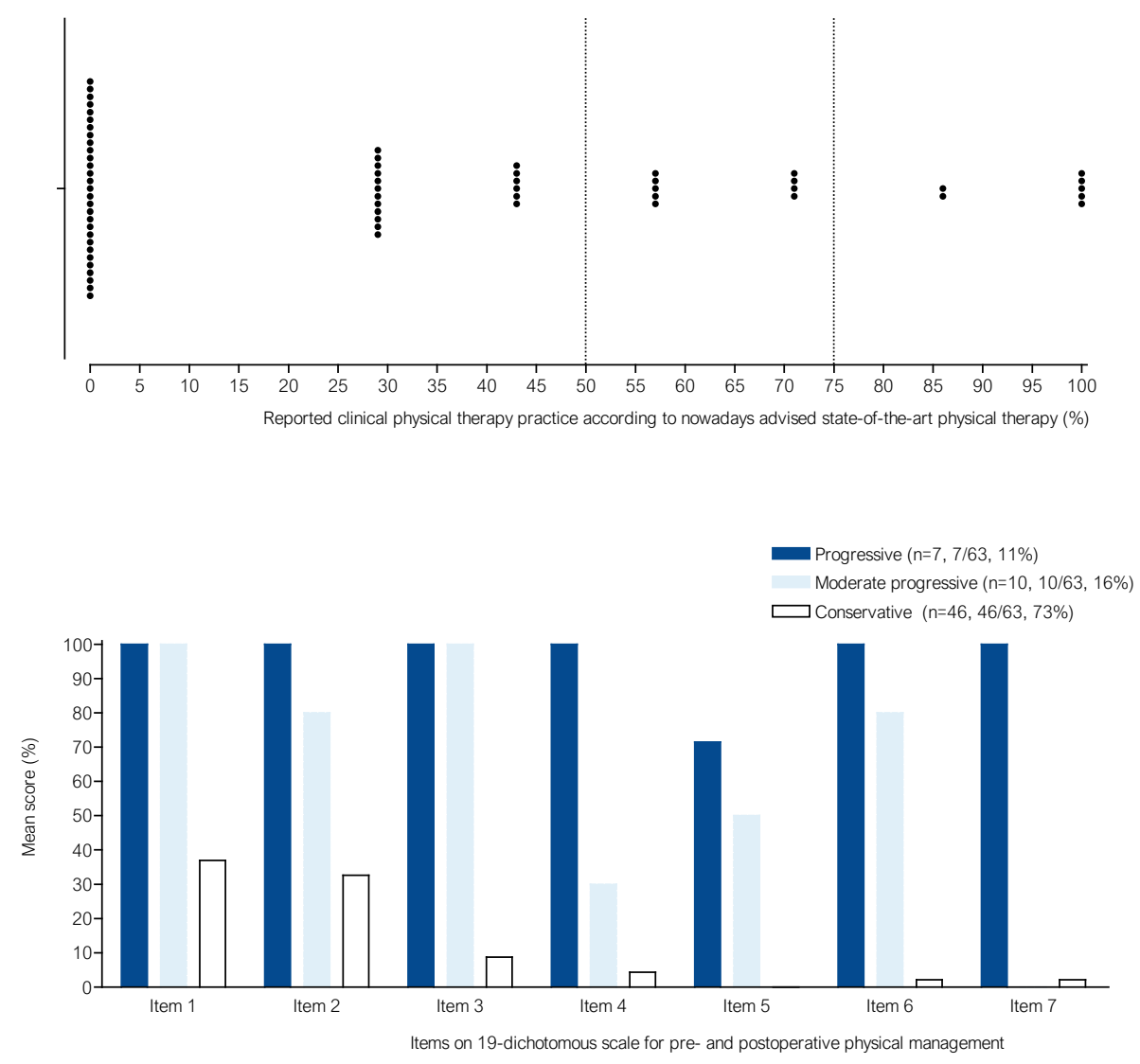

Figure 2.4. The index scores (frequency distribution) calculated on the 7-items for preoperative physical therapy management for each hospital $(n=63)$ (upper panel) and average response profiles for each subgroup on preoperative physical therapy (lower panel). 

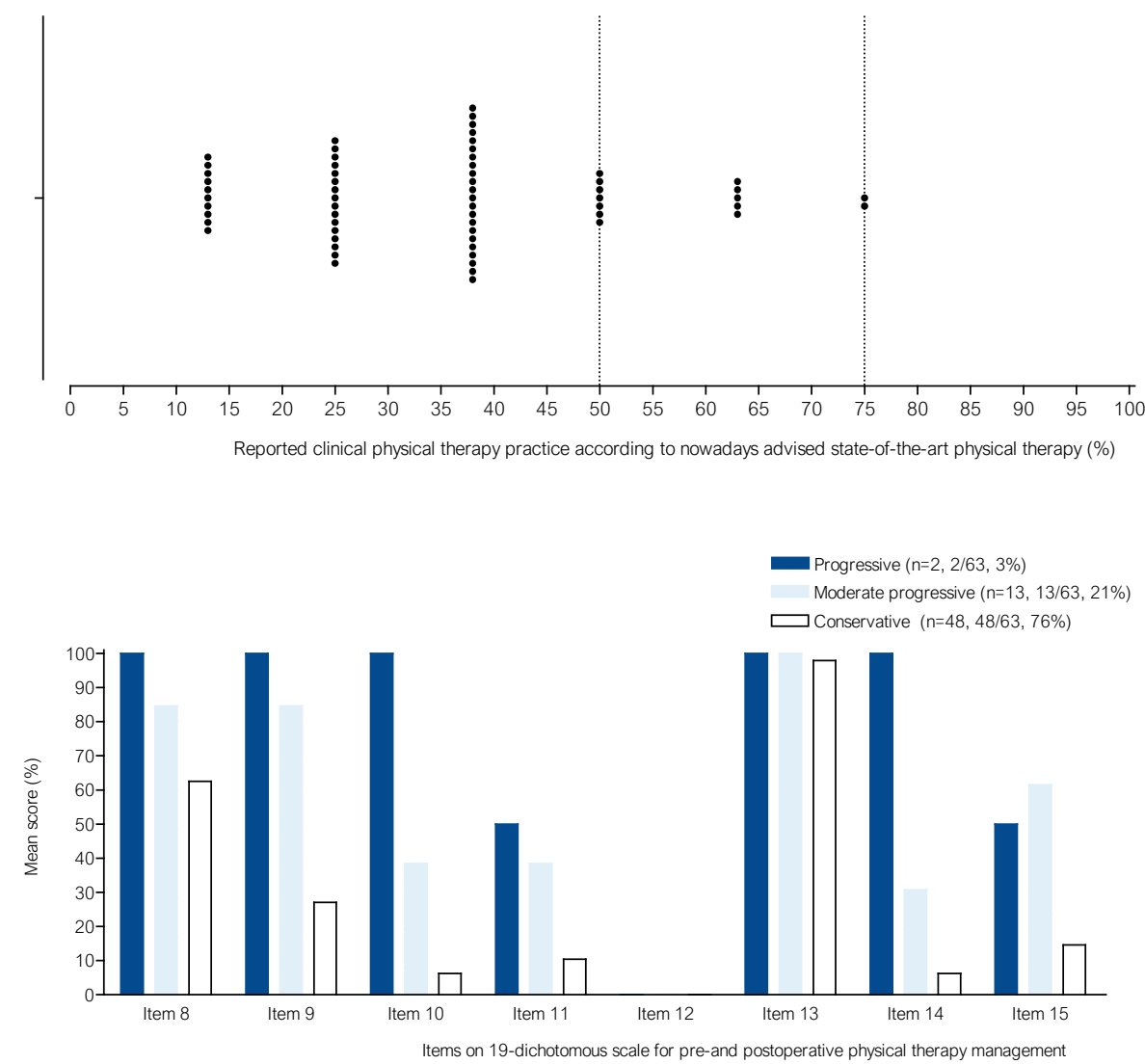

Figure 2.5. The index scores (frequency distribution) calculated on the 8-items for postoperative physical therapy management for each hospital $(n=63)$ (upper panel) and average response profiles for each subgroup on postoperative physical therapy (lower panel). 

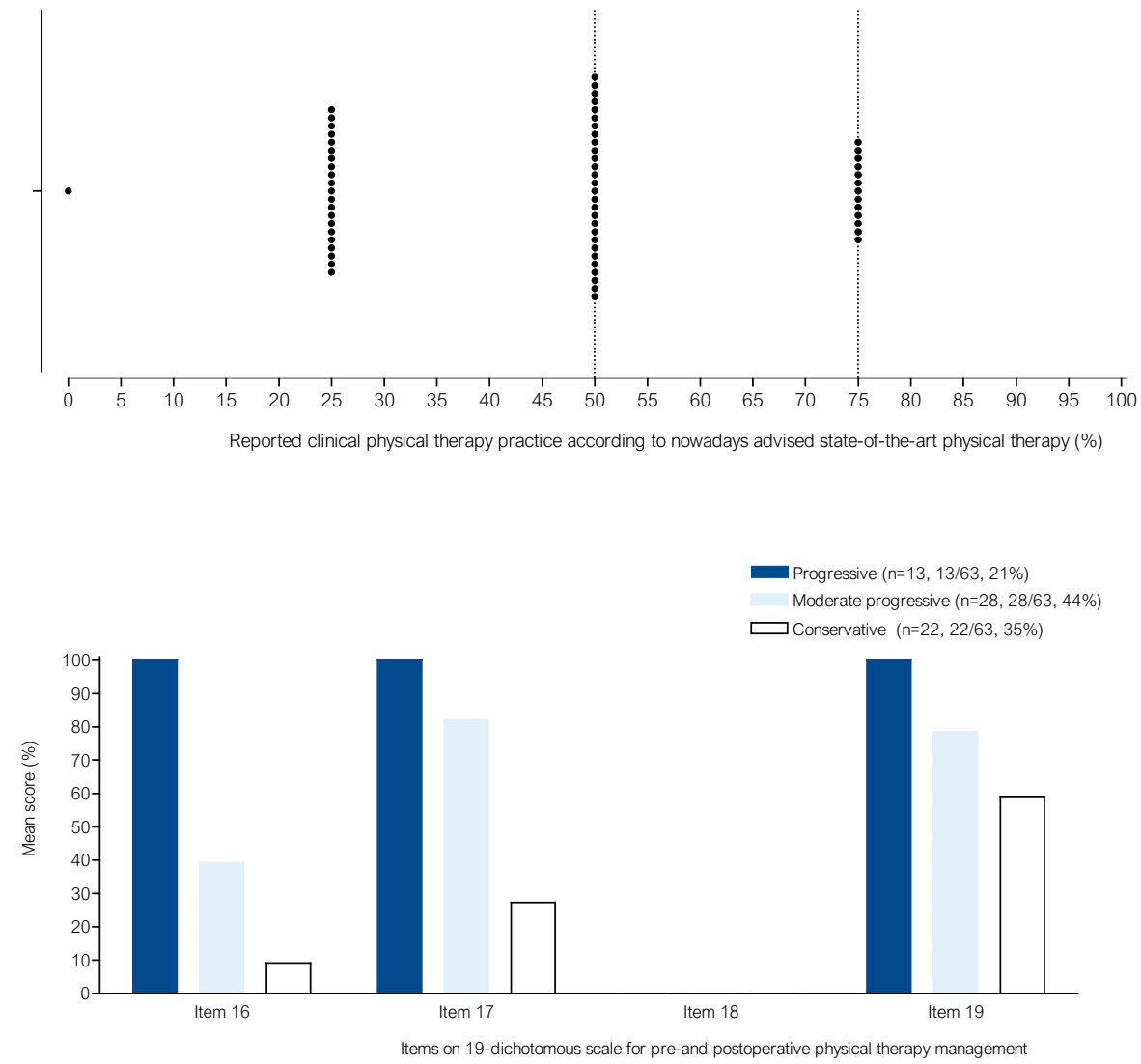

Figure 2.6. The index scores (frequency distribution) calculated on the 4-items for physical therapy management at discharge and readmission for each hospital ( $n=63)$ (upper panel) and average response profiles for each subgroup on physical therapy at discharge and readmission (lower panel). 

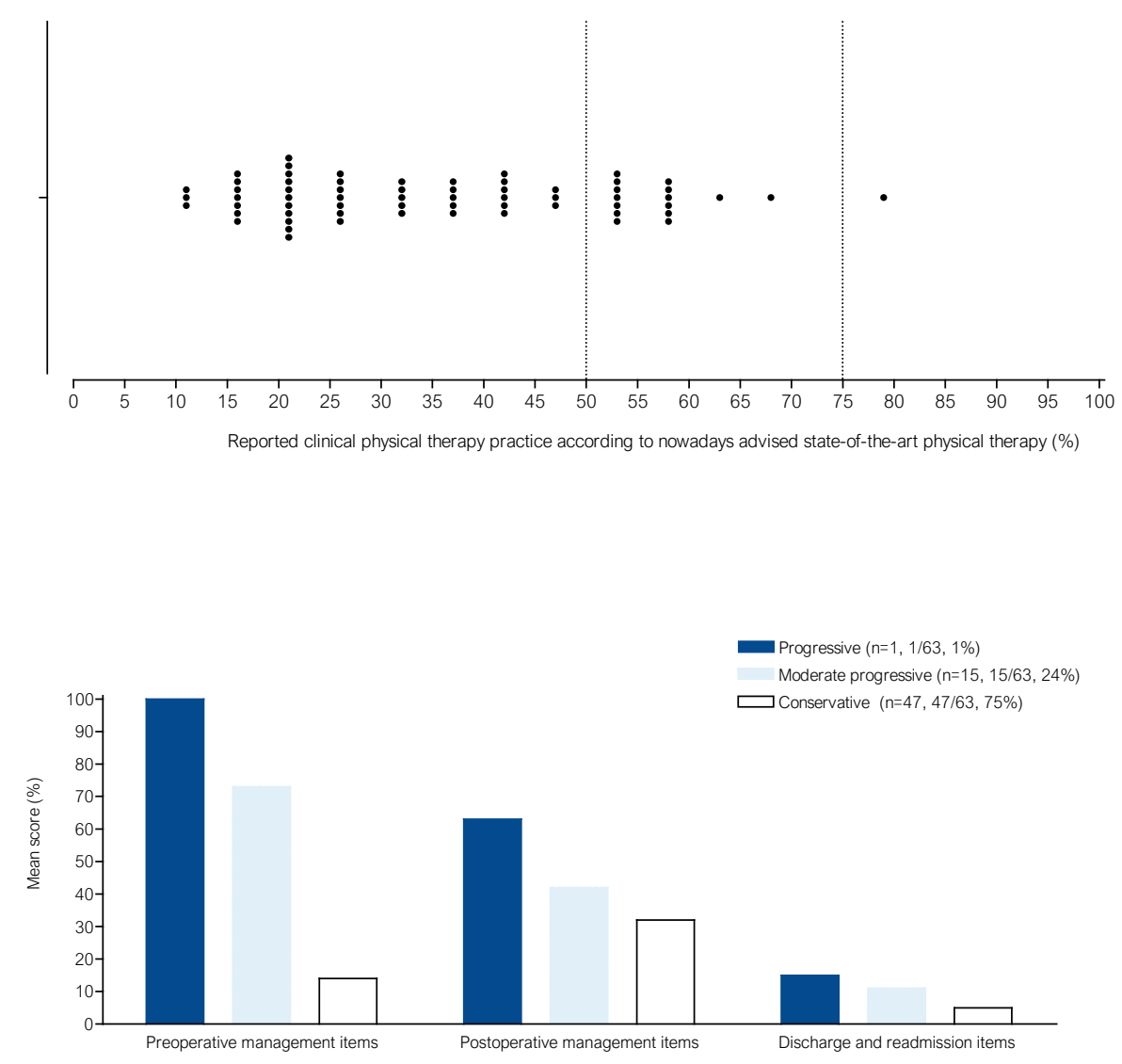

Figure 2.7. The index scores (frequency distribution) calculated on the 19-items for perioperative physical therapy management for each hospital $(n=63)$ (upper panel) and average response profiles for each subgroup on pre-and postoperative physical therapy management (lower panel).

\section{DISCUSSION}

This study provides an overview of the current clinical physical therapy management (response rate $65 / 82,79.3 \%$ ) in patients who opt for abdominal surgery for colorectal, hepatic, or pancreatic cancer in the Netherlands. The reported content and between hospital variability was described, and moderate-to-strong uniformity among physical therapists was reported regarding preoperative education and postoperative physical therapy treatment goals. For pre- and postoperative performance measures, no uniformity was found. In addition, three different classes of clinical physical therapy practice were identified by comparing the nowadays-advised state-ofthe-art physical therapy and the self-reported physical therapy management. 
The first aim of this study was to describe the current content and variability in pre- and postoperative physical therapy management in major abdominal surgery. A response rate $>75 \%$ is considered excellent, which makes the participant responses to our survey fairly representative for the current Dutch clinical practice. Besides, comparable studies reported similar response rates of $80.7 \%$ (46/57) [29] and $82.4 \%$ (28/34) [26]. We consulted physical therapists working on a daily base with patients opting for elective major abdominal surgery and who therefore have extensive knowledge concerning the current practice in their respective hospital. Consequently, this study provides rather accurate information about the current pre- and postoperative physical therapy management in their respective hospitals.

Variability between the respondents was assessed by evaluating the level of uniformity in physical therapy management. In 53.9\% (34/63) of the respondents, patients were seen preoperatively by a physical therapist, in which uniformity existed in the provided elements of education for patients, but not concerning the different domains of physical fitness assessed. Additional descriptive analysis did not demonstrate clear differences between academic and general hospitals. Screening of patients prior to major abdominal surgery by physical therapists is not yet routine physical therapy practice in the Netherlands $[9,10]$. Similar results were reported in surveys in other surgical populations [23,25,30-32]. Previously reported potential barriers for preoperative physical therapy were lack of time, insufficient evidence, or the fact that relevant information was already provided by other health care professionals [32]. Concerning postoperative physical therapy, our study indicated strong uniformity between respondents concerning airway clearing exercises, patient education (stimulating self-management), early mobilization, and practicing functional tasks (e.g., transfers, walking exercises, and stair climbing). Airway clearing exercises were performed in the majority of the hospitals (62/63, 98.4\%); however, the literature remains inconclusive about the type of breathing exercises and the additional effects of breathing exercises compared to mobilization alone in major (upper) abdominal surgery $[33,34]$. Of the respondents, $38.1 \%$ reported that there is no readmission protocol available at their hospital. However, $61.9 \%$ of the respondents is unaware about the availability of such a protocol in their hospital. Nevertheless, $76.2 \%(48 / 63)$ of the respondents reported to be consulted in time when a readmission occurred. It is important to react adequately when a readmission occurs, as a readmission is associated with additional short-term morbidity and mortality in major abdominal surgery [35].

The second aim of the study was to compare the nowadays-advised state-of-the-art physical therapy with the self-reported daily clinical physical therapy practice. Using LCA on the reported 
data about pre- and postoperative physical therapy, three classes of practice were identified, in which hospitals in class I and class II were more likely to provide preoperative risk assessment; however, prehabilitation was most likely to be offered in class I. Therefore, class I is most closely related to the nowadays-advised state-of-the-art physical therapy and are classified as "progressive". Respondents who reported to merely provide preoperative risk assessment are more likely to belong to class II ("moderately progressive"). Respondents that do not provide preoperative physical therapy (risk assessment and prehabilitation) have the biggest chance to belong to class III ("conservative"). These respondents can learn from experiences of class I and II when aiming to implement state-of-the-art evidence- and practice-based preoperative interventions.

The importance of a preoperative assessment of physical fitness in the prediction of postoperative outcomes is well documented nowadays [36]. Cardiopulmonary exercise testing is the gold standard for assessing cardiorespiratory fitness and has been found to have a consistent relation with postoperative outcomes (e.g., morbidity, length of stay, mortality) in major abdominal surgery $[37,38]$. Clear cut-off values for cardiorespiratory fitness to preoperatively identify patients with a higher risk for postoperative morbidity have been published [39]. However, performing cardiopulmonary exercise testing is not always feasible as usual care in the Netherlands. Hereto, more practical performance-based tests to estimate cardiorespiratory fitness can be used (e.g., incremental shuttle walk test, timed up-and-go test) [40,41]; however, they still need further validation [39]. The latter might explain the lack of uniformity between the respondents using these type of tests in their preoperative physical fitness assessment. Moreover, besides measuring cardiorespiratory fitness, it is important to preoperatively assess muscle strength and functional mobility, as these components of physical fitness are a prerequisite for (early) mobilization and being physically active postoperatively [42].

It is encouraging that $17.5 \%$ of the respondents $(11 / 63)$ provide exercise prehabilitation prior to major abdominal surgery. Recent studies in major abdominal surgery demonstrated that prehabilitation (in high-risk patients) improves the preoperative physical fitness level and can reduce the risk for postoperative complications, as well as reduce length of stay [43-45]. Despite these reported beneficial effects, most studies evaluating to identify the effectiveness of prehabilitation hamper preoperative risk assessment that might lead to selection bias (inclusion of mainly low-risk patients) [46]. Moreover, studies are too heterogeneous concerning the context (home-, community-, or hospital-based) and content (frequency, intensity, time, and type) of the preoperative exercise training program, making further research necessary [46]. 
Concerning postoperative physical therapy management, no major differences were seen in the conditional probabilities between the three classes. No probability could be calculated from item 12 and 18, as there was no variability within the item response. Postoperatively, early mobilization and practicing functional task are part of the ERAS care pathway, as implemented in $63.0 \%$ of the respondents. Implementation of ERAS programs resulted in major improvements in clinical outcomes and costs in colorectal, hepatic, and pancreatic surgery [47-49]. Although the importance of early mobilization and the physiological and functional consequences of bed-rest are known [50], it has been reported that patients spent about $83 \%$ of their time in their bed, often without a medical reason [51]. Hereto, adherence to the ERAS care pathway with a multidisciplinary patient-centered approach in combination with a physically activating culture and infrastructure is advocated to improve and accelerate recovery of physical functioning postoperatively $[9,10]$. The low probabilities for the use of performance-based tests to monitor the patient's recovery of physical functioning and the availability of clear (clinimetric) discharge criteria in hospitals is remarkable. Literature describes that frequent monitoring of the recovery of physical functioning, ideally using performance-based tests, during the treatment course is very important for guiding the care process and discharge planning [52].

Implementation of state-of-the-art pre- and postoperative physical therapy management is a complex intervention. A pragmatic strategy in which implementation consists of an interaction between the implementation process, context, and outcomes might be valuable [53]. First, the physical therapist should be aware of the available evidence and the related recommendations for clinical practice. Then, in an iterative process, small changes can be made in daily clinical practice with real-time monitoring of relevant outcomes based on qualitative and quantitative data. This real-time feedback driven adaptations are necessary to make changes in daily clinical practice sustainable $[53,54]$. An example for such an approach is published by van der Sluis et al. [22].

\section{Limitations of the study}

A limitation of this study is uncertainness concerning the generalizability of the used literature to describe the state-of-the-art physical therapy management. Patient characteristics of the participating hospitals were unknown, making it not possible to evaluate how well the patient population described in the literature fits the patient population of the participating hospitals. The survey was not evaluated on reliability and validity and there was no structured assessment on 
the interpretation of the questions. Finally, the use of self-reported answers might lead to bias towards real-life practice.

There is a wide degree of variability between hospitals regarding pre-and postoperative clinical physical therapy practice for patients opting for major abdominal surgery. Three classes of clinical physical therapy practices were identified, differing in adherence to the evidence provided in the literature. It is encouraging to see current developments (e.g., preoperative risk assessment and prehabilitation) in daily clinical practice related to evidence- and practice-based conceptual models as described in the literature to further improve perioperative (physical therapy) care for these patients. Further translation of key research findings into clinical physical therapy practice is advised, especially for hospitals in which the physical therapist is not involved preoperatively. Moreover, improving uniformity by developing up-to-date clinical guidelines is recommended.

\section{ACKNOWLEDGEMENTS}

We would like to thank the Dutch Association for Physical therapy in Hospitals (NVZF), Rudi Steenbruggen and Rik van Hooff, the Dutch Association of Physical therapy Managers (VLF), Bert Risseeuw, for administrative support, and Elise Dusseldorp for statistical advice and all respondents. 
1. World Health Organization. Global health and aging report. 2011. Accessed 31 October 2016, http://www.who.int/ageing/publications/glo bal_health/en/.

2. Makary MA, Segev DL, Pronovost PJ, Syin $\mathrm{D}$, Bandeen-Roche K, Patel P, et al. Frailty as a predictor of surgical outcomes in older patients. J Am Coll Surg. 2010;210:901-8.

3. Yang R, Wolfson M, Lewis MC. Unique aspects of the elderly surgical population: an anesthesiologist's perspective. Geriatr Orthop Surg Rehab. 2011;2:56-64.

4. Lin HS, Watts JN, Peel NM, Hubbard RE. Frailty and post-operative outcomes in older surgical patients: a systematic review. BMC Geriatr. 2016;16:157.

5. Nicholson A, Lowe MC, Parker J, Lewis SR, Alderson P, Smith AF. Systematic review and meta-analysis of enhanced recovery programmes in surgical patients. Br J Surg. 2014;101:172-88.

6. Finnerty CC, Mabvuure NT, Ali A, Kozar RA, Herndon DN. The surgically induced stress response. JPEN J Parenter Enteral Nutr. 2013;37:21S-29S.

7. McEwen BS, Wingfield JC. What is in a name? Integrating homeostasis, allostasis and stress. Horm Behav. 2010;57:105-11.

8. Kim S, Brooks AK, Groban L. Preoperative assessment of the older surgical patient: honing in on geriatric syndromes. Clin Interv Aging. 2014;10:13-27.

9. Hulzebos EH, van Meeteren NL. Making the elderly fit for surgery. Br J Surg. 2016;103:e12-5.

10. Hulzebos EH, van Meeteren NL. Making the elderly fit for surgery. $\mathrm{Br} \mathrm{J}$ Surg. 2016;103:463.

11. Wynter-Blyth V, Moorthy K. Prehabilitation: preparing patients for surgery. BMJ. 2017;358:j3702.

12. Lopopolo RB. Hospital restructuring and the changing nature of the physical therapist's role. Phys Ther. 1999;79:17185.
13. Hood L. Systems biology and P4 medicine: past, present, and future. Rambam Maimonides Med J. 2013;4:e0012.

14. Bongers BC, Dronkers JJ, Hulzebos HJ, Hoogeboom TJ, Buhre WF, van Meeteren NL. Optimizing perioperative physical therapy care in major elective surgery to improve surgical outcome in high-risk patients: the Better in, Better out ${ }^{\mathrm{TM}}$ concept. NTvA. 2016;29:134-9.

15. Tew GA, Ayyash R, Durrand J, Danjoux GR. Clinical guideline and recommendations on pre-operative exercise training in patients awaiting major non-cardiac surgery. Anaesthesia. 2018;73(6):750-68.

16. Lassen K, Soop M, Nygren J, Cox PB, Hendry PO, Spies C, et al. Consensus review of optimal perioperative care in colorectal surgery: Enhanced Recovery After Surgery (ERAS) Group recommendations. Arch Surg. 2009;144:961-9.

17. Lassen K, Coolsen MM, Slim K, Carli F, de Aguilar-Nascimento JE, Schäfer $\mathrm{M}$, et al. Guidelines for perioperative care for pancreaticoduodenectomy: Enhanced Recovery After Surgery (ERAS®) Society recommendations. Clin Nutr. 2012;31:81730.

18. Hulzebos EH, Smit Y, Helders PP, van Meeteren NL. Preoperative physical therapy for elective cardiac surgery patients. Cochrane Database Syst Rev. 2012;11:CD010118.

19. Hulzebos EH, Helders PJ, Favie NJ, De Bie RA, Brutel de la Riviere A, van Meeteren NL. Preoperative intensive inspiratory muscle training to prevent postoperative pulmonary complications in high-risk patients undergoing CABG surgery: a randomized clinical trial. JAMA. 2006;296:1851-7.

20. Dronkers JJ, Lamberts $\mathrm{H}$, Reutelingsperger IM, Naber RH, Dronkers-Landman CM, Veldman A, et al. Preoperative therapeutic programme for elderly patients scheduled for elective abdominal oncological surgery: a randomized controlled pilot study. Clin Rehabil. 2010;24:614-22. 
21. Minnella EM, Bousquet-Dion G, Awasthi R, Scheede-Bergdahl C, Carli F. Multimodal prehabilitation improves functional capacity before and after colorectal surgery for cancer: a five-year research experience. Acta Oncol. 2017;56:295-300.

22. van der Sluis G, Goldbohm RA, Bimmel R, Garre FG, Elings J, Hoogeboom TJ, et al. What augmented physical activity and empowerment can bring to patients receiving total knee replacement: content, implementation, and comparative effectiveness of a new function-tailored care pathway in a routine care setting. BioMed Res Int. 2015:745864.

23. Reeve J, Denehy L, Stiller K. The physiotherapy management of patients undergoing thoracic surgery: a survey of current practice in Australia and New Zealand. Physiother Res Int. 2007;12:5971.

24. Naylor J, Harmer A, Fransen M, Crosbie J, Innes L. Status of physiotherapy rehabilitation after total knee replacement in Australia. Physiother Res Int. 2006;11:35-47.

25. Westerdahl E, Moller M. Physiotherapysupervised mobilization and exercise following cardiac surgery: a national questionnaire survey in Sweden. J Cardiothorac Surg. 2010;5:67.

26. Janssen ERC, Scheijen EEM, van Meeteren NLU, de Bie RA, Lenssen AF, Willems PC, et al. Determining clinical practice of expert physiotherapy for patients undergoing lumbar spinal fusion: a cross-sectional survey study. Eur Spine J. 2016;25:1533-41.

27. Peter WF, Nelissen RG, Vlieland TP. Guideline recommendations for post-acute postoperative physiotherapy in total hip and knee arthroplasty: are they used in daily clinical practice? Musculoskeletal care. 2014;12:125-31.

28. Linzer DA, Lewis JB. poLCA: An R package for polytomous variable latent class analysis. J Stat Softw. 2011;42:1-29.

29. Bougeard AM, Brent A, Swart M, Snowden C. A survey of UK peri-operative medicine: pre-operative care. Anaesthesia. 2017;72:1010-15.
30. Patman S, Bartley A, Ferraz A, Bunting C. Physiotherapy in upper abdominal surgery - what is current practice in Australia? Arch Physiother. 2017;7:11.

31. Cavalheri V, Jenkins S, Hill K. Physiotherapy practice patterns for patients undergoing surgery for lung cancer: a survey of hospitals in Australia and New Zealand. Intern Med J. 2013;43:394-401.

32. Reeve J, Denehy L, Stiller K. The physiotherapy management of patients undergoing thoracic surgery: a survey of current practice in Australia and New Zealand. Physiother Res Int. 2007;12:5971.

33. Reeve J, Boden I. The physiotherapy management of patients undergoing abdominal surgery. New Zealand J Physiother. 2016;44:33-49.

34. Silva YR, Li SK, Rickard MJ. Does the addition of deep breathing exercises to physiotherapy-directed early mobilisation alter patient outcomes following high-risk open upper abdominal surgery? Cluster randomised controlled trial. Physiotherapy. 2013;99:187-93

35. Schneider EB, Hyder O, Wolfgang CL, Hirose K, Choti MA, Makary MA, et al. Patient readmission and mortality after surgery for hepato-pancreato-biliary malignancies. J Am Coll Surg. 2012;215:607-15.

36. Levett DZH, Edwards M, Grocott M, Mythen M. Preparing the patient for surgery to improve outcomes. Best Pract Res Clin Anaesthesiol. 2016;30:145-57.

37. West MA, Asher R, Browning M, Minto G, Swart M, Richardson K, et al. Perioperative Exercise Testing and Training Society (POETTS). Validation of preoperative cardiopulmonary exercise testing-derived variables to predict in-hospital morbidity after major colorectal surgery. Br J Surg. 2016;103:744-52.

38. Levett DZH, Jack S, Swart M, Carlisle J, Wilson J, Snowden C, et al. Perioperative Exercise Testing and Training Society (POETTS). Perioperative cardiopulmonary exercise testing (CPET): consensus clinical guidelines on indications, organization, conduct, and physiological interpretation. Br J Anaesth. 2018;120:484-500. 
39. Moran J, Guinan E, McCormick P, Larkin J, Mockler D, Hussey J, et al. The ability of prehabilitation to influence postoperative outcome after intra-abdominal operation: a systematic review and meta-analysis. Surgery. 2016;160:1189-201.

40. Nutt CL, Russell JC. Use of the preoperative shuttle walk test to predict morbidity and mortality after elective major colorectal surgery. Anaesthesia. 2012;67:839-49.

41. Heldens AFJM, Bongers BC, Lenssen AF, Stassen LPS, Buhre WF, van Meeteren NLU. The association between performance parameters of physical fitness and postoperative outcomes in patients undergoing colorectal surgery: an evaluation of care data. Eur J Surg Oncol. 2017;43:2084-92.

42. Punt IM, Bongers BC, van Beijsterveld $\mathrm{CA}$, Hulzebos HJ, Dronkers JJ, van Meeteren NL. Surgery: moving people, improving outcomes. In: Geriatrics: Hyderabad, India: Avid Science; 2016, p.2-29.

43. Barberan-Garcia A, Ubré M, Roca J, Lacy AM, Burgos F, Risco R, et al. Personalised prehabilitation in high-risk patients undergoing elective major abdominal surgery: a randomized blinded controlled trial. Ann Surg. 2018;267:50-6.

44. Bolshinsky V, Li MH, Ismail H, Burbury K, Riedel B, Heriot A. Multimodal prehabilitation programs as a bundle of care in gastrointestinal cancer surgery: a systematic review. Dis Colon Rectum. 2018;61:124-38.

45. West MA, Wischmeyer PE, Grocott MPW. Prehabilitation and nutritional support to improve perioperative outcomes. Curr Anesthesiol Rep. 2017;7:340-49.

46. Berkel AEM, Bongers BC, van Kamp MS, Kotte $\mathrm{H}$, Weltevreden $\mathrm{P}$, de Jongh FHC, et al. The effects of prehabilitation versus usual care to reduce postoperative complications in high-risk patients with colorectal cancer or dysplasia scheduled for elective colorectal resection: study protocol of a randomized controlled trial. BMC Gastroenterol. 2018;18:29.
47. Ljungqvist $\mathrm{O}$, Scott $\mathrm{M}$, Fearon $\mathrm{KC}$. Enhanced recovery after surgery: a review. JAMA Surg. 2017;152:292-8.

48. Melloul E, Hübner M, Scott M, Snowden C, Prentis J, Dejong CH, et al. Guidelines for perioperative care for liver surgery: enhanced recovery after surgery (ERAS) society recommendations. World J Surg. 2016;40:2425-40.

49. Zhuang $C L$, Ye $X Z$, Zhang $X D$, Chen $B C$, Yu Z. Enhanced recovery after surgery programs versus traditional care for colorectal surgery: a meta-analysis of randomized controlled trials. Dis Colon Rectum. 2013;56:667-78.

50. Sourdet $\mathrm{S}$, Lafont $\mathrm{C}$, Rolland $\mathrm{Y}$, Nourhashemi F, Andrieu S, Vellas B. Preventable iatrogenic disability in elderly patients during hospitalization. J Am Med Dir Assoc. 2015;16:674-81.

51. Brown CJ, Redden DT, Flood KL, Allman RM. The underrecognized epidemic of low mobility during hospitalization of older adults. J Am Geriatr Soc. 2009;57:166065.

52. Glasziou P, Irwig L, Mant D. Monitoring in chronic disease: a rational approach. BMJ. 2005;330:644-8.

53. Luig T, Asselin J, Sharma AM, CampbellScherer DL. Understanding implementation of complex interventions in primary care teams. J Am Board Fam Med. 2018;31:431-44.

54. Moore GF, Audrey S, Barker M, Bond L, Bonell C, Hardeman W, et al. Process evaluation of complex interventions: Medical Research Council guidance. BMJ. 2015;350:h1258. 


\section{APPENDIX}

\section{Appendix 2.1. Questionnaire}

E-survey: evaluating current clinical physical therapy practice for patients undergoing elective colorectal, hepatic, or pancreatic resection.

Additional clarification of the used terminology can be found on the last page.

This e-survey is meant to construct an overview of the current clinical practice of physical therapy in adult patients undergoing elective colorectal, hepatic, or pancreatic resection in the Netherlands. Results will be analyzed anonymously and will be used for publication. We kindly request you to complete this e-survey.

\section{Background information}

1. In what type of hospital do you work?
- Academic hospital
- General hospital
- Specialized health care center
o Other,

2. What is the name of the hospital where you work?

3. Is a protocol available at your institution for treating patients undergoing major elective abdominal surgery?

$\circ$ Yes

$\circ$ No

4. Are you willing to make your hospital's protocol for treating patients undergoing major elective abdominal surgery available to us? The document will be analyzed anonymously. If yes, please provide us with your e-mail address, so we can contact you.

- Yes, my e-mail address is

$\circ$ No

○ Not applicable 
5. How many physical therapists work at the physical therapy department of your hospital?

__ physical therapists with a Bachelor of Applied Science degree physical therapists with a Bachelor of Applied Science and a clinical specialization (e.g., oncology, pediatrics)

_ _ physical therapists with a Master of Science degree

_ physical therapy students

Preoperative physical therapy management

6. Are patients undergoing major elective abdominal surgery preoperatively seen by a physical therapist at your hospital?

o Yes

$\circ$ No

7. Are all patients preoperatively seen by a physical therapist?

o Yes

$\circ$ No, only when referred by a specialist physician, nurse practitioner or case manager

8. What is the content of the preoperative physical therapy intervention for patients undergoing elective major abdominal surgery (multiple answers possible)?

o Patient education

- Preoperative assessment of physical functioning

- Exercise prehabilitation

9. Patient education consists of (multiple answers possible)?

o Information leaflet related to physical therapy during the hospitalization phase

- The option to ask questions

- Education about breathing exercises

- Education about circulation exercises

○ Other, namely 
10. Which components of physical fitness are assessed preoperatively (multiple answers possible)?

- Daily physical activity level

- Peripheral muscle strength

- Inspiratory muscle strength

- Range of motion (passive range of motion, active range of motion)

o Functional mobility (transfers, walking, climbing stairs)

- Cardiorespiratory fitness

- Pain (perceived level of pain during physical activity)

○ Cognition

o Fatigue (perceived level of fatigue during physical activity)

o Other, namely

11. Are all patients eligible for exercise prehabilitation?

o Yes

- No

12. How do you decide whether a patient is eligible for exercise prehabilitation?

13. What type of physical exercise training is provided during exercise prehabilitation (multiple answers possible)?

○ Inspiratory muscle strength training

- Peripheral muscle strength training

- Cardiorespiratory exercise training

- Functional exercise training

o Other, namely

14. Where does exercise prehabilitation take place (multiple answers possible)?

○ At the patient's home

- At a local physical therapy private practice

○ At the hospital 
15. Do you make use of any specific performance tests during the preoperative physical therapy visit at your hospital (e.g., ten-meter walk test, steep ramp test, timed up-and-go test)? If yes, please describe them down below.

○ Yes, namely

$\circ$ No

16. Do you make use of any specific questionnaires during the preoperative physical therapy visit at your hospital? If yes, please describe them down below.

$\circ$ Yes, namely

$\circ$ No

17. How often do you see a patient during the preoperative period?

o Once

$\circ$ Twice

- Three times

- Four times or more

\section{Postoperative physical therapy management}

18. Are there any care pathways implemented at your hospital for the postoperative rehabilitation procedure of a patient who underwent major elective major abdominal surgery (e.g., "fast-track", "enhanced recovery after surgery", "Better in, Better out")?

○ Yes, namely

$\circ$ No

19. Was the physical therapist involved in the development of this care pathway?

o Yes

$\circ$ No

20. Do all patients who underwent major elective abdominal surgery receive postoperative physical therapy (usual care)?

- Yes

o No 
21. What is the reason that not all patients receive postoperative physical therapy (usual care)?

○ It depends on the type of surgery

o It depends on the referral of the ward physician or surgeon

o Other, namely

22. Who is responsible for the involvement of the physical therapy during the postoperative period at your hospital (multiple answers possible)?

$\circ$ The surgeon

- The ward physician

○ The nurse

○ The physical therapist

- The nurse practitioner or case manager

o Other, namely

23. How many days postoperatively is a patient who underwent major elective abdominal surgery first seen by the physical therapist?

- Postoperative day 0 (at the day of surgery)

- Postoperative day 1 (the first day after surgery)

- Postoperative day 2

- Postoperative day 3

○ It depends on a referral for physical therapy

o Other, namely

24. On average, what is the duration of a postoperative physical therapy session? minutes

25. What is the location at which most patients participate in postoperative physical exercise training?

$\circ$ In the patient room at the ward

○ At the department of physical therapy

$\circ$ In a living room

o Other, namely 
26. Can you give a breakdown of percentages concerning the location at which most patients participate in postoperative physical exercise training by shifting the bars (the sum of the three percentages should be 100)?

$$
\begin{array}{lllllllllll}
0 & 10 & 20 & 30 & 40 & 50 & 60 & 70 & 80 & 90 & 100
\end{array}
$$

Room at the nurse department

Department physical therapy

The living room

27. How frequently are patients who underwent major elective abdominal surgery seen postoperatively by physical therapy?

- Once a day

- Twice a day

- Three times a day

o Other, namely

28. Can the physical therapy treatment frequency be adjusted when desired?

o Yes

$\circ$ No

29. Are patients who underwent major elective abdominal surgery structurally seen during weekends?

○ Yes, on both weekend days

○ Yes, only on one weekend day

$\circ$ Yes, only when there is enough time

- Yes, only in case of postoperative pulmonary complications

$\circ$ No

o Other, namely 
30. What is the content of postoperative physical therapy interventions for patients who underwent major elective abdominal surgery (multiple answers possible)?

- Breathing exercises

- Circulation exercises

- Practicing transfers

- Walking (with or without walking aid)

- Stair climbing

- Peripheral muscle strength exercises

- Cardiorespiratory exercise training

o Patient education related to mobilization during hospital stay

o Patient education homework exercises

31. Are there any other interventions the physical therapist performs postoperatively in daily clinical practice that are not yet mentioned in the previous question?

- Yes, namely

o No

32. Do you (structurally) use validated questionnaires during the clinical postoperative physical therapy treatment of patients who underwent major elective abdominal surgery?

o Yes

o No

33. Do you (structurally) use validated performance tests during the clinical postoperative physical therapy treatment of patients who underwent major elective abdominal surgery?

$\circ$ Yes

$\circ$ No

34. Which performance tests do you use postoperatively (multiple answers possible)?

○ Six-minute walking test (6MWT)

- Timed up-and-go test (TUG)

- lowa level of assistance scale (ILOA)

- Modified lowa level of assistance scale (MILAS)

- De Morton mobility index (DEMMI)

- Handgrip strength

- Chair-stand Test 
○ Inspiratory muscle strength

o Other, namely

\section{Discharge and readmission}

35. Who decides whether a patient is ready for hospital discharge?

○ The ward physician

○ The surgeon

- The physical therapist

- Shared decision-making

36. Are specific discharge criteria used at your hospital for patients who underwent major elective abdominal surgery?

$\circ$ Yes, namely

- No

37. What is the most frequent hospital discharge destination of patients who underwent major elective abdominal surgery at your hospital?

○ Home

- Rehabilitation center

- Nursing home

38. Are you called consulted in time in case of a readmission of a patient who underwent major elective abdominal surgery?

$\circ$ Yes

o No

39. Down below you will see two statements. Please, indicate whether these statements apply to your hospital concerning a readmission protocol for patients who underwent major elective abdominal surgery.

At my hospital, a readmission protocol is available

Yes No Unknown

At my hospital, the readmission protocol is followed

○ $\quad \circ$

○ $\quad \circ$ 
40. On a scale from 0 to 10 , how useful do you consider a readmission protocol for patients who underwent major elective abdominal surgery?

\begin{tabular}{|c|c|c|c|c|c|c|c|c|c|}
\hline 0 & 1 & 2 & 3 & 4 & 5 & 6 & 7 & 8 & 9 \\
\hline 0 & 0 & 0 & 0 & 0 & 0 & 0 & 0 & 0 & o \\
\hline
\end{tabular}

41. Are you willing to make your hospital's readmission protocol for patients who underwent major elective abdominal surgery available to us? The document will be analyzed anonymously. If yes, please provide us with your e-mail address, so we can contact you.

$\circ$ Yes, my e-mail address is

o No

- Not applicable

This is the end of the e-survey, thank you for your participation. If there are any questions related to this e-survey, please provide them below or send us an e-mail.

\section{Further clarification of terminology}

"The option to ask questions" (question 9)

Components of patient education. Was patient education provided in one direction only (providing information to the patient by the physical therapist), or was it also possible for patients to interact with the therapist, to ask questions, and express concerns?

\section{"Walking" (question 10 and question 30)}

Teaching the patient how to ambulate with or without a walking aid as part of the physical therapy intervention postoperatively.

\section{"Physical fitness, physical activity, cardiorespiratory fitness" (question 10)}

Physical activity is any body movement produced by the skeletal muscles that result in an increase in energy expenditure [1]. Here we mean the behavior of individuals that encompasses participating in activities of daily life such as household activities, walking, leisure activities, sport participation, et cetera. Physical fitness is the ability to perform aspects of sports, occupations or activities of daily life. Cardiorespiratory fitness is a health-related component of physical fitness, and is the maximal capacity body's circulatory and respiratory systems to supply oxygen to skeletal muscles during physical activity/exercise. Cardiorespiratory fitness can directly be 
measured by a cardiopulmonary exercise test as maximal oxygen consumption ( $\left.\mathrm{VO}_{2 \max }\right)$ or can be estimated as the exercise capacity (maximal work rate) from an exercise test.

\section{"Usual care" (question 20)}

Although the definition of usual care has not been standardized, it can include the routine care received by patients for prevention or treatment of diseases [2]. Applied to this survey question it refers to: is it standardized in the route daily care practice that patients receive physical therapy after surgery or is it something that only is giving at a special indication. In other words: does just every patient receive postoperative physical therapy, or is this decided for each individual patient separately?

\section{References appendix 2.1.}

1. Caspersen CJ, Powell KE, Christenson GM. Physical activity, exercise, and physical fitness: definitions and distinctions for health-related research. Public Health Rep. 1985;100:126-31.
2. Harlapur M, Shimbo D. Usual Care, in Encyclopedia of Behavioral Medicine. Springer New York. 2013. p.2021-2022.

\section{NOTES}


Een embedded science benadering is waardevol om duurzaam veranderingen te realiseren voor predictieve, preventieve en gepersonaliseerde perioperatieve fysiotherapeutische zorg. 


\section{Chapter 3}

Improvement of preoperative and postoperative performance of physical functioning in patients undergoing elective hepato-pancreato-biliary surgery: initiation of a regime transition

Christel A. van Beijsterveld | Bart C. Bongers |Marcel den Dulk | Lieke J. Corpelijn | Cornelius H. Dejong | Nico L. van Meeteren 


\section{Background}

The aim of this study was to provide insight into the initiation and development phase of the transition of perioperative physical therapy management towards a predictive, preventive, personalized, and participatory (P4) health care approach in a university medical center in the Netherlands. Here, P4 perioperative physical therapy management aims to preserve or even improve pre- and postoperative physical functioning of high-risk patients that decided to have major elective abdominal surgery because of liver or pancreatic malignancies.

\section{Methods}

An observative single setting transition design with a concurrent transformative mixed-method strategy was used in the perioperative hepato-pancreato-biliary care pathway for almost 4 years (2015-2018). Each previously mentioned 'P' of the P4-concept was seen as a 'value' stream. Actions considered as strategically relevant within a particular value stream were monitored by an embedded scientist, who also collected all types of quantitative and qualitative data.

\section{Results}

In the predictive stream, an outpatient preoperative assessment unit was developed and herewith a perceived level of physical functioning, functional mobility, age and type of resection were identified as patient's preoperative predictor variables for short-term (30 days) postoperative outcomes. A regional prehabilitation network of physical therapists $(n=28)$ was developed and hospital strategies (infrastructural, culture, practices) were developed to improve a patient's preand postoperative physical functioning. An e-health application and the concept of 'titration' were introduced in practices in the participatory and personalized stream. In total, 174 patients who underwent pancreatic or hepatic resection were monitored pre- and postoperatively for their level of physical functioning. Median time to recovery of physical functioning decreased (2 days in pancreatic resection and 1 day in hepatic resection) and median length of hospital stay decreased as well ( 4 days in pancreatic resection and 1 day in hepatic resection).

\section{Conclusion}

The initiation of a transition towards P4 perioperative physical therapy care mainly resulted in changes in the predictive and preventive care stream followed by the personalized stream. Coinciding with the transition experiment, a decrease in time to recovery of physical functioning and length of hospital stay was observed in patients that decided to undergo pancreatic or hepatic resection. Further exploration and evolvement of this perioperative transition, ecological validation, and creation of a sustainable new perioperative physical therapy care pathway is advocated. 


\section{INTRODUCTION}

Surgical resection remains the cornerstone of curative treatment for cancer in the liver, pancreas, and biliary tract. In recent decades, advances in hepato-pancreato-biliary (HPB) surgery and anesthesia have resulted in improved perioperative management and surgical outcomes, even for elderly patients [1, 2]. For patients, their families, and clinicians, decision-making for surgery and perioperative care is challenging. Especially in frail patients, the presence of comorbidities, age-related psychophysiological changes, and a diminished physiological reserve to withstand the stress of the pathology, diagnosis, neoadjuvant therapies, hospitalization, surgery, and periand postoperative complications are all considerable risk-factors and stressors. Risk factors and stressors affect a patient's physical functioning, an indicator that should be taken into account in the decision to opt for surgery [3-8]. A substantial proportion (30\%) of patients $>70$ years experiences a decrease in physical functioning due to their hospital stay and surgery, which negatively affects their recovery of physical functioning and performance of activities of daily life $[9,10]$. Furthermore, the risk for morbidity and mortality in HPB surgery is high, especially for pancreatic surgery (about 60.0\% and 2.9-3.5\% in 2017 [11], parameters known to be related to physical fitness [12].

Over the recent decennia, overall ageing of the population and technological improvements in surgical and anesthetic techniques have resulted in an older population undergoing surgery. In this context, a transition in the continuum of perioperative care has been initiated by surgeons and anesthesiologists (e.g., enhanced recovery after surgery [ERAS] and fast-track programs) in order to reduce mortality and morbidity rates. Likewise, experts have advocated to optimize preand postoperative physical therapy management to avoid the negative consequences of hospitalization and surgery on a patient's daily (physical) functioning. For instance, Hulzebos \& Van Meeteren described a proactive perioperative physical therapy management strategy that drives patient-centered and collaborative care, aiming for optimal physical fitness and functioning before, during, and after hospitalization for major surgery $[7,8]$. The proposed strategy follows the concept "P4 health" [13], a concept in which the delivered perioperative care should be predictive, preventive, personalized, and participatory [14].

This transition in perioperative care towards the P4-concept implies adopting recent evidencebased insights. Based on these, fundamental changes in the practices (professional procedures and protocols), culture (professional's paradigms, values, knowledge), and structures (e.g., the ward, outpatient clinic, hospital infrastructure, inter-institutional networks, medical technologies) 
should be made in order to work via the P4-concept towards a function-tailored perioperative care pathway. Such a pathway at the same time preferably uses the current evidence-base for the relation between the preoperative level of physical functioning and postoperative outcomes [15] and focusses consequently on a patient's physical functioning $[7,8]$ besides their health as such.

In the scope of 'science in transition' [16] and based upon the rather conservative perioperative practice of physical therapy in the Netherlands $[17,18]$, the research approach of transition by an embedded scientist seems profitable amongst others [19-22]. For instance, Van der Sluis et al. [20] have shown the beneficial accomplishments of using an observative transition design by an embedded scientist in the facilitation, construction, monitoring, and evaluation towards a new function-tailored clinical care pathway in an orthopedic hospital ward [20]. Inspired by the P4 concept, the notion of the association between physical fitness and surgical outcomes, and encouraged by success of the methodological approach of Van der Sluis et al. [20], a transition experiment was performed to improve perioperative physical therapy management in patients that decided to have major elective abdominal surgery because of hepatic or pancreatic malignancies.

\section{METHODS}

Study design

In this prospective, observational, single-setting transition design, a concurrent transformative mixed-method approach was used by an embedded scientist. To describe, monitor, and evaluate the pre-development phase of the HPB perioperative care transition in the clinical care pathway, an in-depth multistage mixed-method framework was followed. By meticulously monitoring relevant functional, medical, surgical, contextual, and patient-related factors, the (medical and functional) course of individual patients over time was evaluated. Ethical approval for this study was obtained by the medical ethical committee of the Maastricht University Medical Center+ (154-234).

\section{Setting and participants}

This transition experiment was performed in coalition with the departments of HPB surgery and physical therapy at the Maastricht University Medical Center+. The health care team that was responsible for the treatment of patients scheduled for elective hepatic or pancreatic surgery included surgeons, nurse practitioners, nurses, anesthesiologists, dieticians, and physical 
therapists. All were involved in the transition process towards P4 perioperative physical therapy management. Other health care professionals (e.g., members of the inpatient pain team, psychologists) were available as consultants/advisors when necessary. Patients, health care providers, and researchers were closely working together during the execution of the transition experiments. An embedded scientist (CB) was working as a physical therapist (0.5 fte, Department of Physical Therapy, Maastricht University Medical Center+) and researcher ( $0.5 \mathrm{fte}$, Department of Epidemiology, Maastricht University) at the HPB surgical ward, and was responsible for the initiation and execution of the interventions and the monitoring of the results of the transition process. The aforementioned team was involved in reflection upon the interventions, results, and their mutual interpretations.

\section{Theory: transformative organizational lens}

A transformative organizational lens was developed as the predominant perspective (see Figure 3.1). This lens assembles the theory concerning transition management and methodology [23] with professional concepts of proactive perioperative physical therapy management strategies $[7,8]$. It provides a framework on how to integrate proactive perioperative physical therapy management strategies in daily clinical practice, while simultaneously evaluating whether these concepts are gradually paying off in this specific context (ecological validation).

\section{Practice: transition structure}

A transition is a purposeful change-trajectory over time, characterized by systemic changes in the dominant culture, structure, and professional and customer (here: the patient) practices in a societal sub-system [23]. A system is an institution (here, the Maastricht University Medical Center+) designed to meet general societal and specific clinical needs, for instance serving society and delivering health care (see Figure 3.1, $\square$ blue box) [24]. To meet societal needs, several solutions are present in the system that involves tools (technology and infrastructure) and processes (health care practices) (see Figure 3.1, $\mathrm{O}$ blue circles). Furthermore, people in the system (actors) may up-held certain values that are embodied to the system, called streams [24] (see Figure 3.1, $\nabla$ grey arrow). Streams are values based on current knowledge (e.g., made available by science, clinical experience, technology), whereas actors in the system have the ability to affiliate with a particular stream influencing their networks and practices. De Haan \& Rotmans [24] proposed that a transformation in a system - here the hospital setting - is the result of strategic actions by various people (actors) who hold new values about practices in the system and who align in an innovated particular stream (see Figure 3.1, $\boldsymbol{\nabla}$ blue arrow). Streams can 
be shared by different actors who may join forces to formulate and collaborate on an idea, which may lead to a solution (see Figure 3.1, $\square$ orange box) that emerges in a system or leads to a new system. Those new solutions will lead to a change in culture, structure, and practices. Within such a transformation, actors can have different roles depending on their values and position in a system; frontrunners, connectors, topplers, and supporters [24].

\section{Practice: organization of the transition process within the 'selected' structure}

To structure the transition in a routine perioperative care setting, each of the four 'P's (predictive, preventive, personalized, and participatory) described for perioperative care [14] were seen as a 'value' stream outside the current system (here, the hospital) (see Figure 3.1, $\boldsymbol{\nabla}$ ). During the process of transition, the embedded scientist analyzed the routine perioperative care setting (the current clinical practice, structure, and culture, focused at physical therapy management). Moreover, the embedded scientist aimed to establish connections between the four perioperative care streams and the relevant actors in the system in order to create alliances to change the system. Lastly, the embedded scientist acted as a change agent by initiating and facilitating experiments in line with the four streams, while simultaneously observing and evaluating the effects of these experiments on the system (for example by meticulously monitoring the level of physical functioning of patients during the perioperative period). For each stream, the role of different actors, alliances, and the proposed solutions leading to structural changes in the current system were described in terms of changes in clinical practice (e.g., health care practices), culture (e.g., the prevailing perspective, paradigms), physical structure (e.g., the ward, different departments), and social structure (e.g., networks, organizations). 

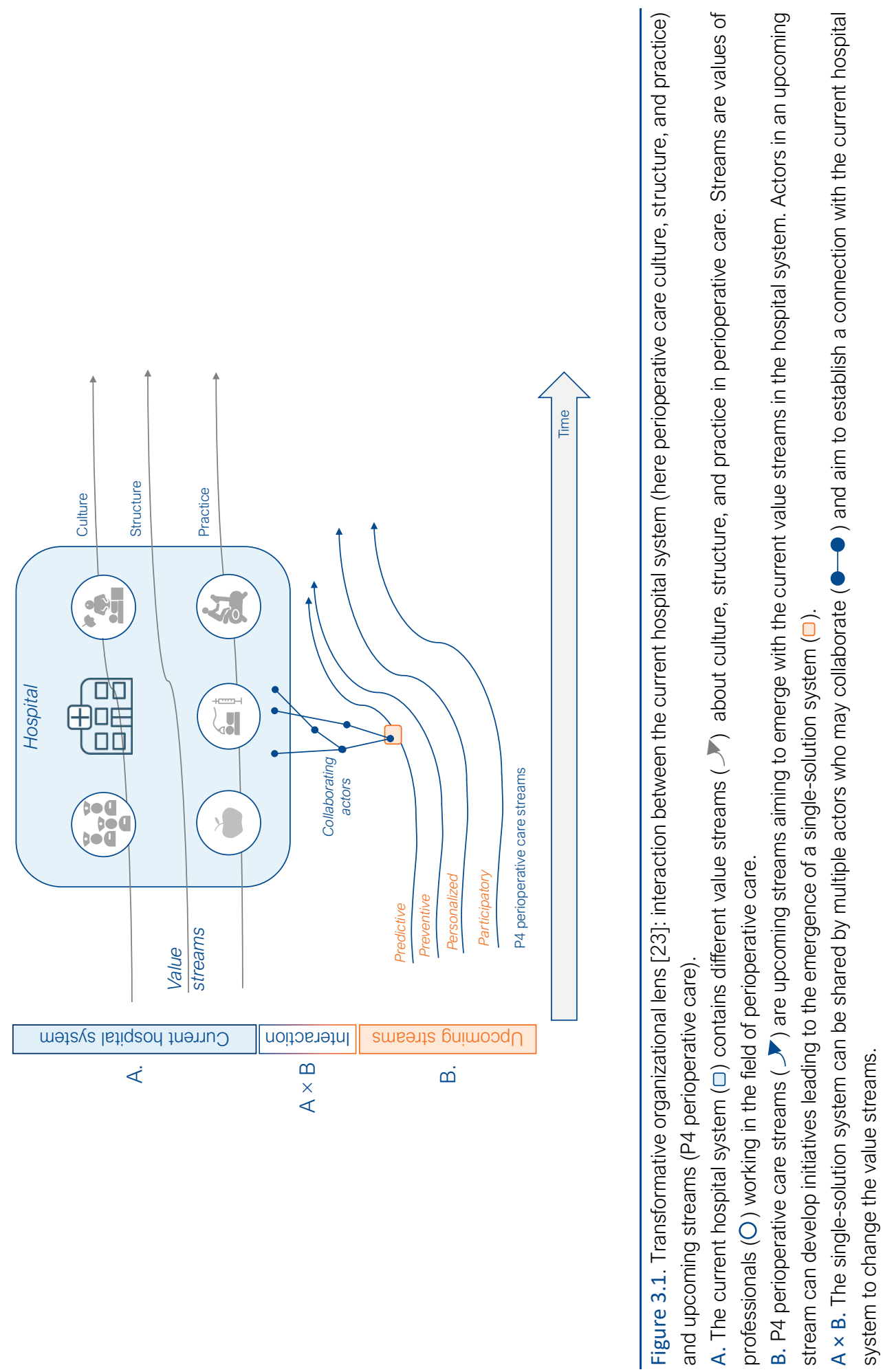


\section{Chapter 3}

\section{Monitoring}

\section{System}

The transition process was monitored by meticulously collecting all types of quantitative and qualitative data during the course of the transition trajectory (see Figure 3.2). The purpose of monitoring was to evaluate changes over time in hospital culture, structure, and practices. Combining these changes with relevant outcomes in the main streams of P4 perioperative care provided insight in the dynamics of the transition. Data were collected between October 2015 and December 2018. A combination of quantitative (e.g., demographics, measurements of physical fitness and physical functioning, surgical outcomes, postoperative recovery of physical functioning, postoperative complications) and qualitative measures (e.g., contributions of the actors, their thoughts, and contextual characteristics of the system in which the experiment was delivered) were collected.

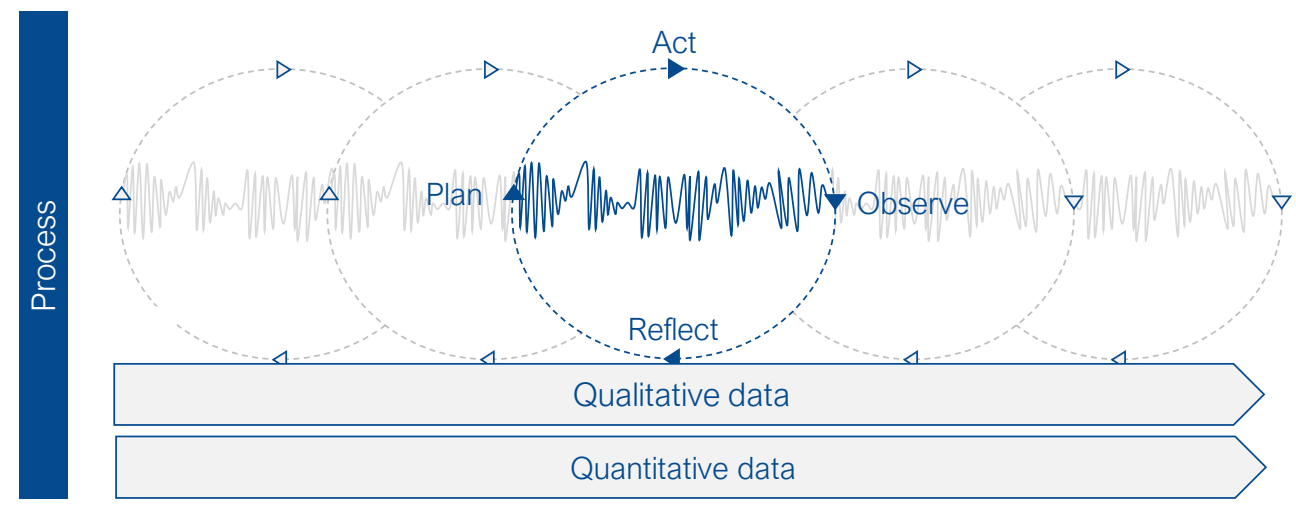

Figure 3.2. An embedded scientist coordinated the process and analyzed the transition in the perioperative care system by following the plan-act-observe-reflect cycle. Within this process the scientist gathered qualitative and quantitative data. Based on these data, the embedded scientist acted as a change agent in initiating and facilitating the transition experiment. 


\section{Streams}

The experiments performed in each stream were monitored by gathering qualitative and quantitative data. Each ' $P$ '-stream contains the following content; 1) predictive (preoperative screening to predict a patient's perioperative risks); 2) preventive (preoperative interventions as physical exercise training, nutritional support, and psychological support in at risk patients to enhance their coping with the hospitalization and surgical stress, thereby facilitating recovery of physical functioning postoperatively); 3) personalized (tailored pre- and postoperative interventions to improve patient adherence and treatment outcomes); and 4) participatory (encouraging patients to actively participate in their treatment by self-care and self-management) $[7,8]$.

\section{Patients}

To measure a patient's level of physical fitness and functioning, the following performance-based tests were used: the steep ramp test (SRT) for aerobic capacity, handgrip strength (HGS) for muscle strength, and the timed up-and-go (TUG) test, two-minute walk test (2MWT), and five times sit-to-stand (FTSTS) test for functional mobility. In addition, the perception of functional capacity to perform activities of daily life was evaluated by using the veterans-specific activity questionnaire (VSAQ) and Duke activity status index (DASI). Postoperative recovery of physical functioning was monitored on a daily base using the modified lowa level of assistance scale (mILAS).

\section{Analyses}

Analyses of the transition in the perioperative care system were performed using a single-case study approach, in which the case here is the complete perioperative trajectory and setting as a whole. Information from the qualitative data was used with the aim to develop a chronological description of the changes in the perioperative care system. The described theoretical framework of the transition structure was applied to describe the dynamics of the transition - patterns of the different streams, the roles of the actors, proposed solutions - in the perioperative care system over time. Additionally, the iterative nature of data collection and analysis allowed to reflect critically with the interdisciplinary team on the results, after which implementation strategies were changed when necessary. The quantitative data was connected with the qualitative description of changes in the perioperative care system. To test between-group differences, independentsamples t-tests or Mann-Whitney U-tests were performed, as appropriate. Univariable and multivariable logistic regression analysis was performed to assess the association between 
preoperative predictor variables and postoperative complications, delayed recovery of physical functioning, and prolonged hospital length of stay. Dependent continuous variables (time to recovery of physical functioning and length of hospital stay) were dichotomized according to their respective median value. To select potential predictor variables for the multivariable regression model, a P-value $<0.100$ was used. For the other statistical analyses, a P-value $<0.05$ was considered statistically significant.

\section{RESULTS}

The system

Since 2001, one of the co-authors and co-founder of ERAS (CD) successfully implemented the ERAS concept [25] to optimize pre-, peri- and postoperative care at the surgical ward, along with other perioperative innovations such as Early Rescue of the anesthesiology. In the prevailing care pathway, patients were preoperatively seen by the surgeon, anesthesiologist, and nurse specialist. The hospital culture (e.g., beliefs and attitudes) and infrastructure induced sedentary behavior, since the patient's room consisted mainly of a bed surmounted with a television. Physical therapists were usually merely involved in postoperative care on request of the surgeon; however, close monitoring of a patient's level of physical functioning was not yet performed.

The perioperative care for patients undergoing HPB surgery changed throughout the three years and four months, and pre- and postoperative monitoring of a patient's level of physical functioning is part of routine care nowadays. Home-based exercise prehabilitation is available when patients are classified as high-risk for adverse postoperative outcomes. Postoperatively, physical therapy is initiated at postoperative day 1 , of which the volume and duration depend on the patient's level of physical functioning and comorbidities. Median time to recovery of physical functioning and median length of hospital stay declined between 2016 and 2018 (see Table 3.1). 
Table 3.1. Outcomes of patients who underwent hepatic and pancreatic resection between 2016 and 2018.

\begin{tabular}{|c|c|c|c|c|c|c|}
\hline \multicolumn{7}{|c|}{ Hepatic resection } \\
\hline & \multicolumn{2}{|r|}{2016} & \multicolumn{2}{|r|}{2017} & \multicolumn{2}{|r|}{2018} \\
\hline & $n$ & median (IQR) & $n$ & median (IQR) & $n$ & median (IQR) \\
\hline $\begin{array}{l}\text { Recovery of physical } \\
\text { functioning (days) }\end{array}$ & 57 & $3.0 \quad(4.0)$ & 42 & $3.0 \quad(4.0)$ & 33 & $3.0 \quad(3.0)$ \\
\hline $\begin{array}{l}\text { Length of hospital } \\
\text { stay (days) }\end{array}$ & 57 & $7.0 \quad(6.0)$ & 42 & $7.0 \quad(4.0)$ & 33 & $6.0 \quad(4.3)$ \\
\hline \multicolumn{7}{|c|}{ Pancreatic resection } \\
\hline & \multicolumn{2}{|r|}{2016} & \multicolumn{2}{|c|}{2017} & \multicolumn{2}{|r|}{2018} \\
\hline & $n$ & median (IQR) & $n$ & median (IQR) & $n$ & Median (IQR) \\
\hline $\begin{array}{l}\text { Recovery of physical } \\
\text { functioning (days) }\end{array}$ & 20 & $8.0 \quad(10.0)$ & 29 & $6.0 \quad(5.0)$ & 20 & $6.0 \quad(6.0)$ \\
\hline $\begin{array}{l}\text { Length of hospital } \\
\text { stay (days) }\end{array}$ & 20 & $15.0(17.0)$ & 29 & $11.0(18.0)$ & 20 & $11.0(13.0)$ \\
\hline \multicolumn{7}{|c|}{$\begin{array}{l}\text { Data are presented as median values and interquartile range. } \\
\text { Abbreviations: IQR=interquartile range. } \\
\text { a. measured by the modified lowa level of assistance scale. }\end{array}$} \\
\hline
\end{tabular}

\section{Streams}

\section{Predictive}

The embedded scientist initiated the stream of predictive perioperative physical therapy care, aiming to identify risk factors for a delayed postoperative recovery of physical functioning. In September 2015, the embedded scientist acquired knowledge concerning available risk prediction models in the literature for patients undergoing HPB surgery. Although cardiopulmonary exercise testing is the gold standard for the assessment of aerobic capacity [12, $26,27]$ and aerobic capacity is independently related with postoperative outcomes in major abdominal surgery, the equipment was not yet available in the physical structure at that time at the department of physical therapy or the department of HPB surgery of the Maastricht University Medical Center+. Hence, the aim was to develop a hospital-specific preoperative risk prediction model focused on pre- and postoperative physical functioning by using the available facilities and equipment at that time.

In October 2015, the embedded scientist contacted the department of HPB surgery concerning the development of an outpatient preoperative assessment unit (see Figure 3.3, '15 Q3). The nurse specialist (LC) acted as the connector between patients visiting the HPB surgical department and the physical therapist. Together they formed a small social structure (network) 
operating segregated from the usual care pathway, with little cooperation amongst other professionals. They organized the logistics, information leaflets, and materials to develop the preoperative assessment unit (see Figure 3.3, '16 Q1). In March 2016, a physical structure (e.g., a high-end cycle ergometer to perform exercise testing) was available and practices changed. The outpatient preoperative assessment was now performed at the department of physical therapy. After the decision for surgery, patients undergoing HPB surgery were directly guided to the physical therapy department for the preoperative screening of their physical fitness and physical functioning. After six months, the attained social structure was expanding their views on how to further improve perioperative practices. Connections were made with the department of dietetics and the department of internal medicine (geriatric medicine) to discuss logistical issues for organizing referrals and outpatient clinics. This movement resulted in changes in the social structure and practices in June 2017; the outpatient preoperative assessment was extended with a preoperative screening of nutritional status and a preoperative geriatric assessment by the nurse specialist. When indicated from the screening results, patients could be referred for further assessment or interventions when indicated (see Figure 3.3, '16 Q3). Despite these efforts, not all patients (30\%) visited the outpatient preoperative assessment unit before surgery, due to the administrative burden and lack of awareness by surgeons. This resulted in the fact that not all patients for hepatic resection were routinely screened. Therefore, the embedded scientist initiated the development of a new information communication technology infrastructure in which the digital order for surgery was automatically generating an order for the outpatient preoperative assessment clinic in February 2018. At the same time, a multidisciplinary perioperative care working group was initiated for patients undergoing major abdominal surgery, leading to a circle of support with a new social structure (network of hospital physical therapists, nurses, surgeons, dieticians, an exercise physiologist, anesthesiologist, and internist with a focus on geriatric medicine; see Figure 3.3, '18 Q1-2). The circle of support jointly explored new opportunities by three one-hour group sessions to connect the current predictive stream with an upcoming stream in the hospital about how to organize the logistics of the outpatient clinics. Even though frontrunners, connectors, and supporters were included in the perioperative care arena, there was no toppler (in the sense of a sustainable implementation strategy by the management team) to anchor the outpatient preoperative assessment unit in the emerging hospital system as part of the usual care activities. 


\section{Preventive}

'I am literally lying in bed waiting for the moment that my stomach starts working again, I have no idea how to spend my time in the hospital'

- 62-year-old man with gastroparesis following pancreatic surgery

In January 2016, the embedded scientist was approached by a nurse at the HPB surgical ward to think about the development of a decision aid for patients and nurses. The underlining idea was to create a shift in culture concerning the value of early mobilization in relation to a patient's recovery of physical functioning. This resulted in two posters that were operationalized in May 2016 (see Figure 3.4, '16 Q2), one for patients and one for nurses to increase the awareness of the importance of early mobilization. After three months, the subjective observation of the hospital physical therapists and nurses was that there was still no change in culture. Hence, a new social structure was built with two hospital physical therapists and two unit leaders of the surgical ward to define bottom-up strategic actions to increase awareness regarding the importance of a physically activating culture at the ward. 

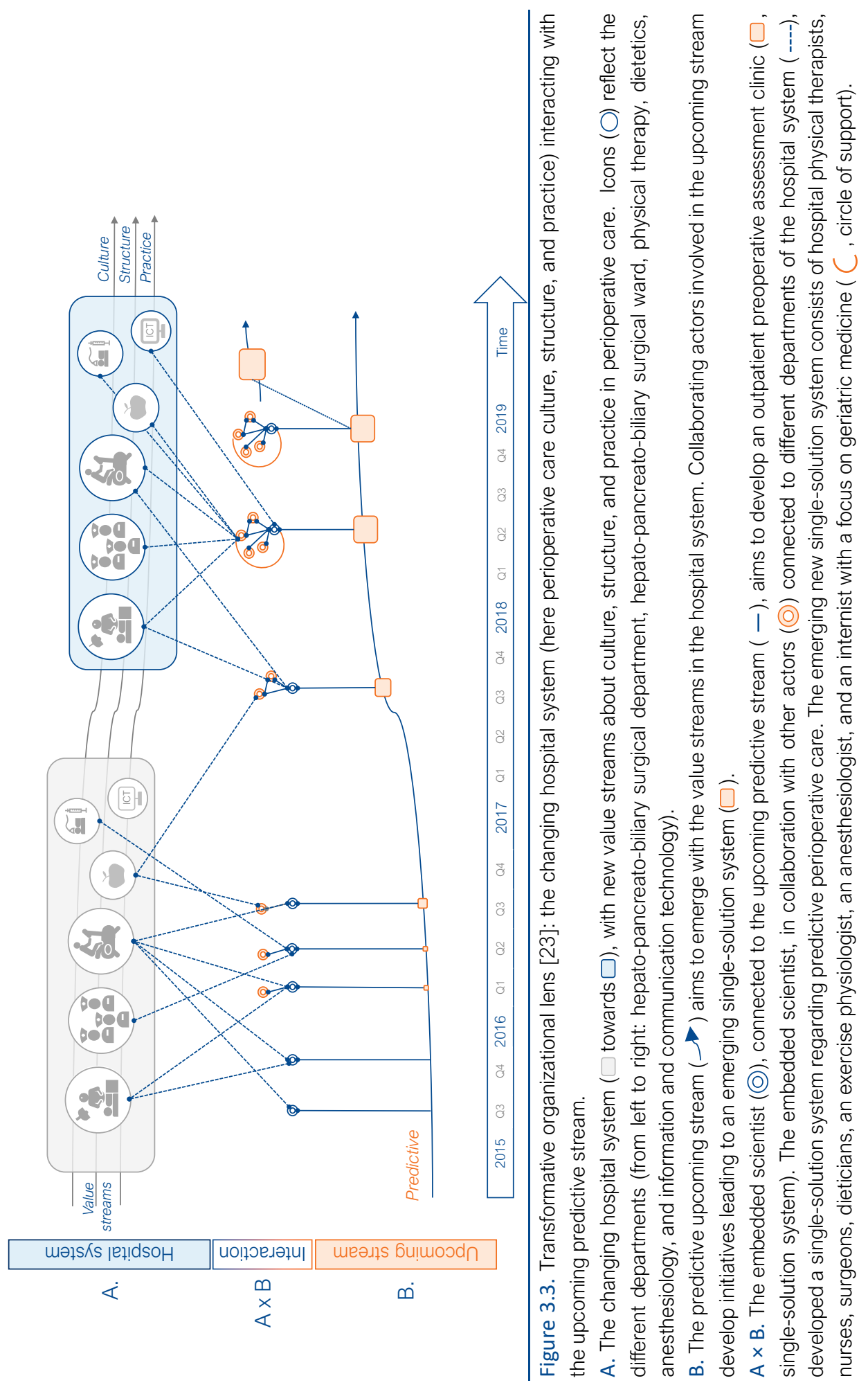
This resulted in diverse actions in the second and third quarter of 2017 (see Figure 3.4, '17 Q23). Patient observations and accelerometers were used to monitor the physical activity level of patients who underwent pancreatic or hepatic surgery $(n=18)$ by physical therapists $(n=2)$. Postoperatively, the median duration of physical activity was 12 minutes on day 1 (ranging from 1.0 to 69.0 minutes) and 33 minutes at day six (ranging from 7.0 to 55.0 minutes). Patients were most active during self-care activities and during physical therapy sessions. Based on these measurements, further actions were developed consisting of 1) optimization of patient information leaflets, 2) education sessions for physical therapists and nurses about the importance of early mobilization, 3) a visual representation of the effects of hospitalization and surgery on a patient's physical functioning (see Figure 3.5), and 4) a new living room for patients in which they could socialize, for instance by having dinner and sitting and chatting together and with people who came to visited them. In practices this resulted in annual education sessions for nurses working at the gastrointestinal surgical wards (including the HPB surgical ward), optimized preoperative information for patients as provided by the nurse specialist, and a referral for physical therapy at the day of admission or the day of surgery to start physical therapy treatment as soon as possible postoperatively.

Perioperative dynamics changed because of the approval of a business plan by the hospital management in the third quarter of 2017 . The embedded scientist became responsible for the development of a decision-support guideline. Supported by a frontrunner (NM) with expertise on functional task training in the home-based setting [28]. The embedded scientist acted as a connector and aligned the value stream of the outpatient physical therapy practices and the value stream of the frontrunner, resulting in a mutual stream. In practices the outcome was a preoperative decision-aid to preserve and/or improve a patient's preoperative physical fitness and physical functioning. The preoperative decision-aid was presented during a three-day course for physical therapists ( $n=28$ ) working within the catchment area of the hospital (30 km), who aligned themselves with the preventive stream for providing home-based prehabilitation. As of December 2018, the multidisciplinary perioperative care workgroup was connected with the prehabilitation network of physical therapists (see Figure 3.4, '18 Q4). The embedded scientist was responsible for the initiation of a home-based prehabilitation program in high-risk patients based on the preoperative screening. 

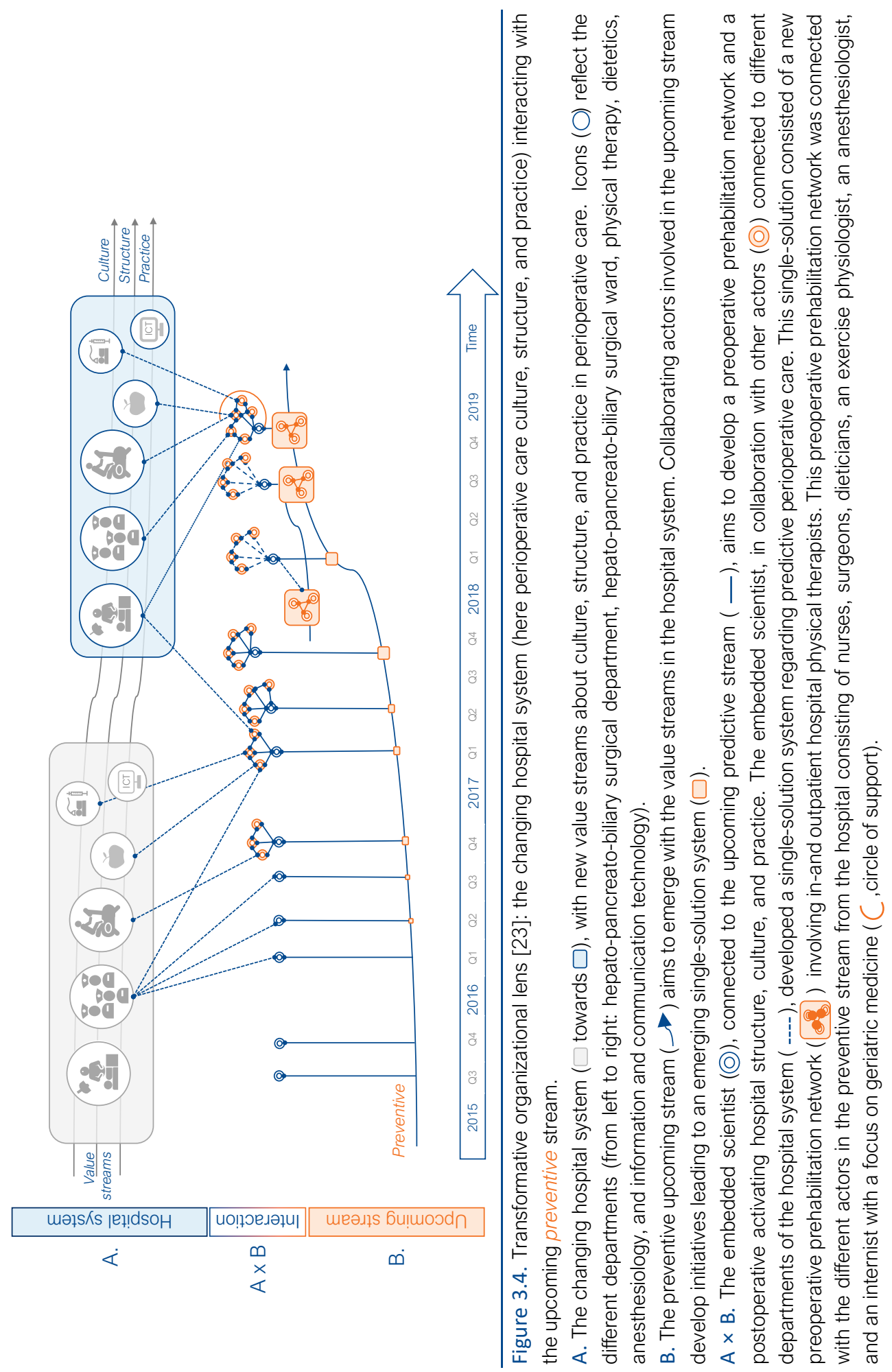

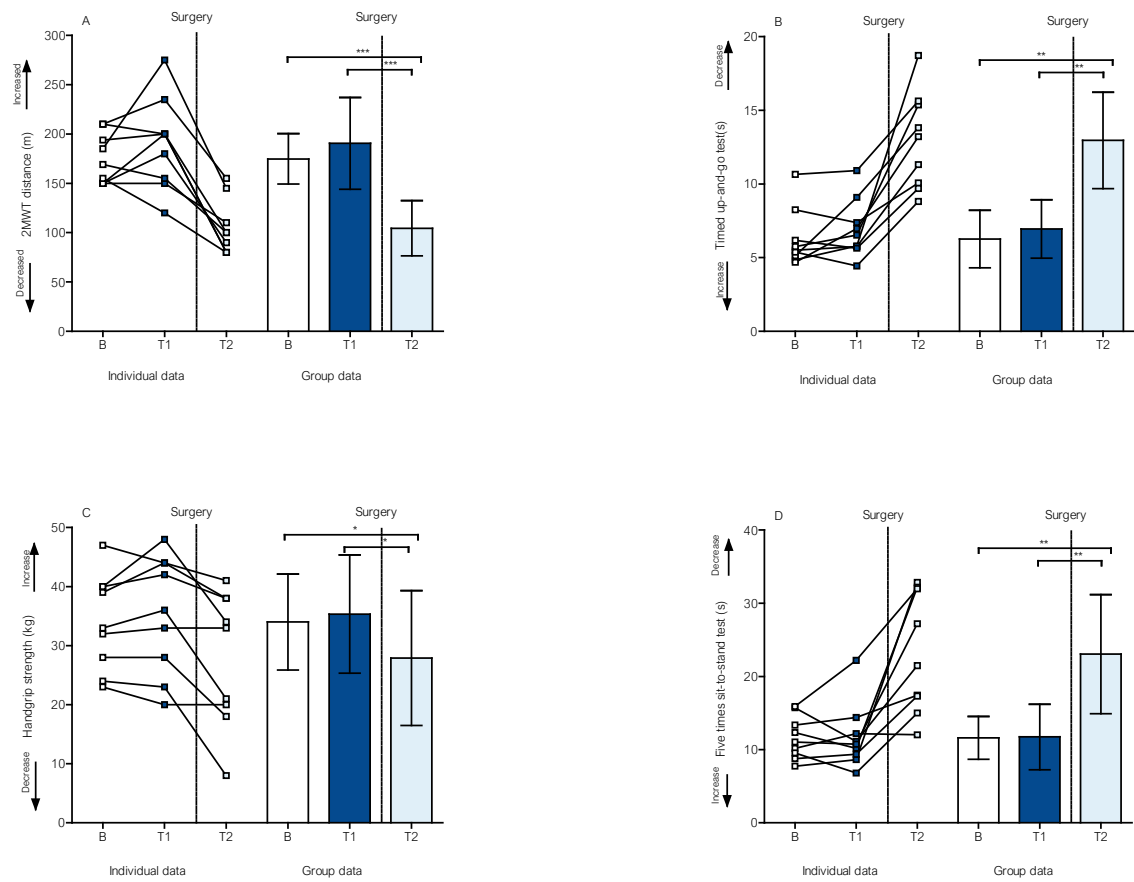

Figure 3.5. Patient's level of physical functioning measured with four different performance-based tests before and after hospitalization and hepatic resection, both on the individual (lines) and group (bars) level $(n=8)$. Reading guide single graphs for instance A. Performance on two-minute walk test (2MWT): great variability in two-minute walking distance between individuals regarding their baseline measurement four weeks before surgery (B) and one day before surgery (T1). All patients experience a decrease walking distance at discharge (T2). At group level, small increase in walking distance in the time before surgery. Significant decrease in walking distance after hospitalization and surgery.

Abbreviations: $\mathrm{B}=$ baseline measurement approximately four weeks before surgery, $\mathrm{T} 1=$ one day before surgery), T2=discharge *: $\mathrm{P}<0.05 ;{ }^{* *}: \mathrm{P}<0.01{ }^{* * *}: \mathrm{P}<0.001$. Data-collected in 2017, Q2-3.

\section{Personalized}

Between November 2015 and August 2016, the embedded scientist acted as a connector to implement the mILAS as a tool to objectively monitor a patient's recovery of physical functioning on a daily base (see Figure 3.6, '15 Q4 - '16 Q3). By using different strategies (e.g., oral presentations, digital information boards, flash-cards, discussion with colleagues, education of students), the use of the mILAS to track a patient's recovery of physical functioning became anchored in the institutional context of the HPB surgical ward. 
Meanwhile, between March 2016 and December 2016 a group of frontrunners (physical therapists and the embedded scientist) analyzed the organization of the clinical practices of the department of physical therapy and proposed a new model with a broad perspective on the strategic, tactic, and operational level to the management of the physical therapy department. Their model had wide implications for everyday physical therapy practice and individual patients, as it reorganized the logistics, initiated patient-tailored (volume of) physical therapy and aimed to improve the collaboration and communication with the other health care professionals at the clinical wards. Although a pilot was performed at the department of orthopedics between March 2017 and September 2017, the stream dried up because of a switch in the management team of the physical therapy department and a new strategy for the department physical therapy (see Figure 3.6, '17 Q3-4).

Furthermore, in July 2017, the concept of 'titration', adopted from Glasziou et al, 2005 [29], was introduced into practices, in both the pre- and postoperative phase. Titration supports intervention management of training by monitoring of patient's level of physical functioning and shared decision-making by patient and therapist about for instance the intensity/dose, content and context of choice of the physical therapy. Preoperatively, titration was part of objectively monitoring training progression during home-based prehabilitation. Postoperatively, daily scores on the mILAS were plotted over time to monitor recovery of physical functioning. In combination with the incidence of a clinical event (e.g., complication, reintervention) the mILAS score showed the physical therapist how to optimally adapt therapy (in frequency, intensity, type of exercise, and duration) (see Figure 3.7). However, this has not been widely accepted in the current physical therapy practices. 

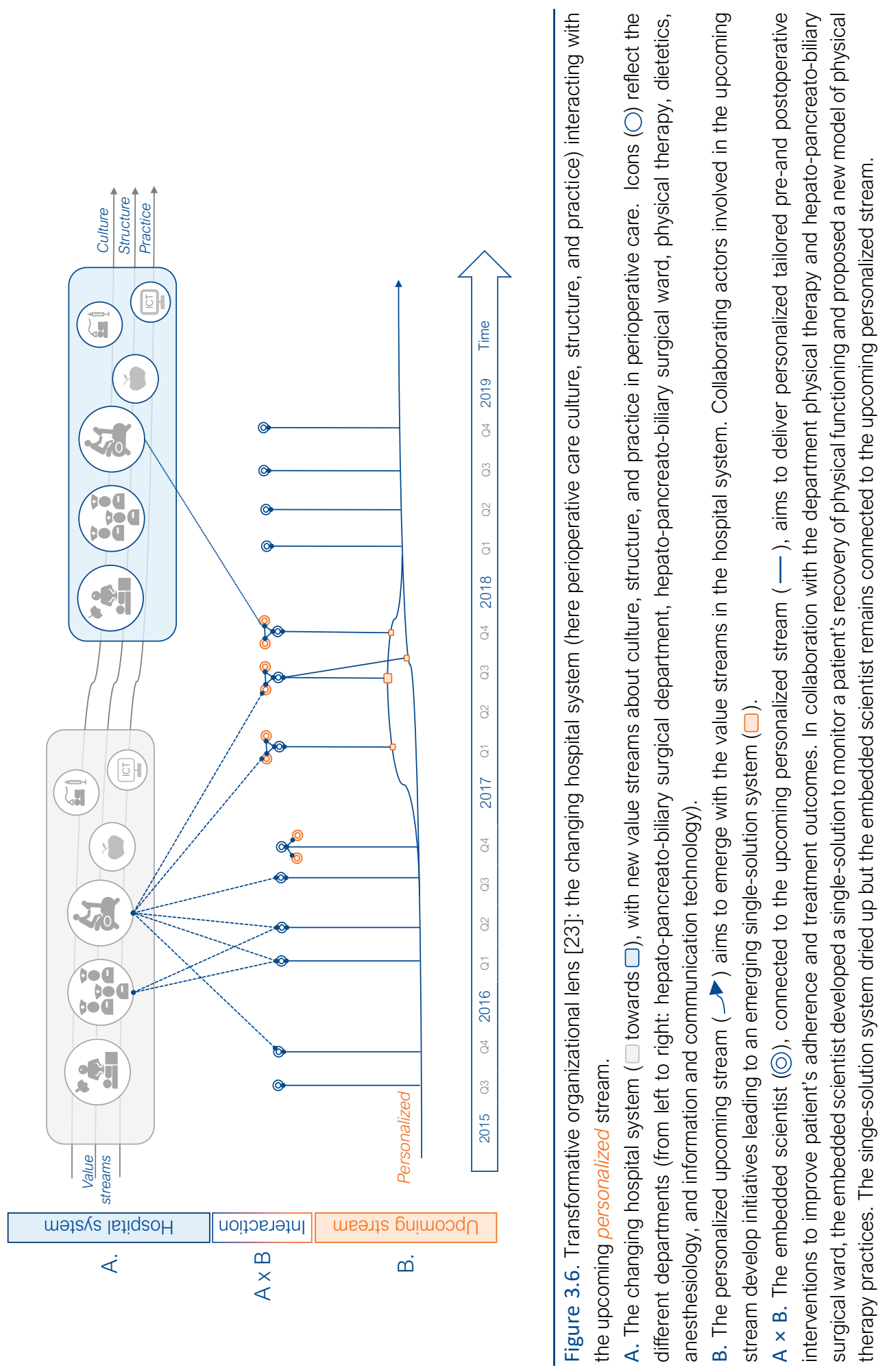

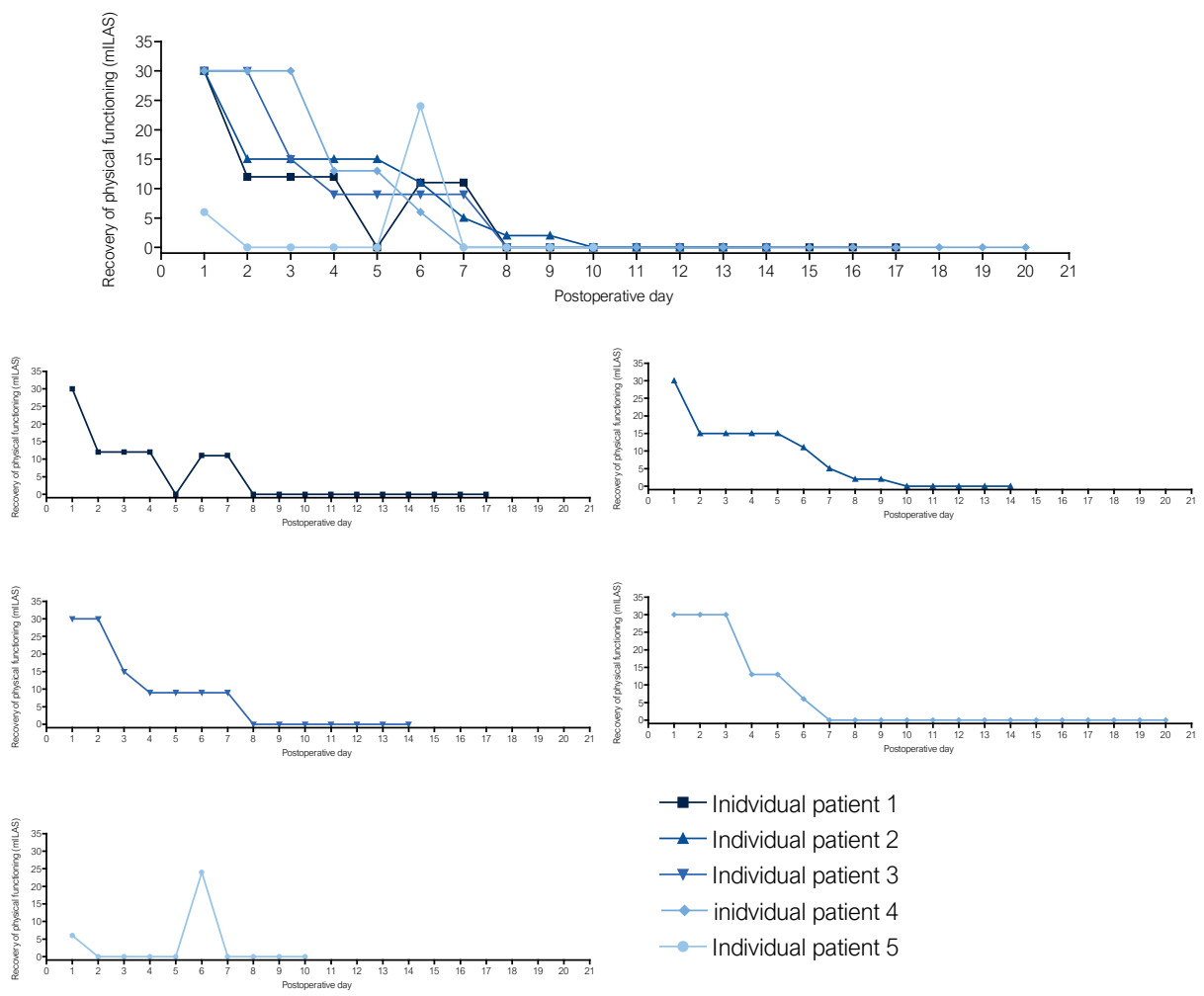

Figure 3.7. Between-patient variability $(n=5)$ in recovery of physical functioning after hepatic or pancreatic resection. Recovery of physical functioning was monitored using the mILAS score, ranging from 30 (fully dependent) to 0 (fully independent) in performing five functional tasks.

Abbreviation: mILAS=modified lowa level of assistance scale.

\section{Participatory}

In 2015, a frontrunner (NM) posted in the support team (embedded scientist and physical therapists) the question "How can we as health care professionals get ourselves being involved in a patient's daily physical functioning, instead of involving a patient in our daily practice or research?" This question and its corresponding provoked thoughts were all about patient participation.

In March 2016, physical therapists were educated on how they could support patients to develop knowledge, confidence, and skills to make well-informed decisions and to initiate pre- and postoperative actions that might improve their postoperative recovery of physical functioning. Furthermore, the embedded scientist designed an e-health application consisting of an 
information portal, insight in the patient's physical functioning, insight in the patients' activity level, and tips for specific exercises. In the years that followed, a partnership was formed with a private partner, funding was obtained for the development of the e-health application, and pilot testing was performed. In November 2018, preparations were made for the implementation of the e-health solution in clinical practice. Meanwhile, alternative initiatives were explored, including 1) a new information letter to inform patients about their preoperative screening results, 2) workbook for patients on how to remain physically active during the hospitalization phase, and 3) graphical visualizations of a patient's recovery of physical functioning (see Figure 3.7). These initiatives were a success in terms of experimenting and discussing possible solutions with different patients. As a result, the three initiatives changed drastically over time, thereby incorporating the feedback of the involved patients. Despite this, these initiatives failed in terms of policy implementations and therefore did not actually improve a patient's active participation in preoperative optimization for surgery and postoperative recovery of physical functioning.

"Nice visualization, I think I am improving in my recovery of physical functioning, as the line is increasing, but based on what? I will show this to my children, they will certainly understand it" 81-year-old woman following pancreatic surgery.

\section{Patients}

Between March 2016 and December 2018, 231 patients were preoperatively screened by the physical therapist. Not all perioperative data of 33 (14.3\%) patients with hepatic resection were yet available for analysis, 24 (9.5\%) patients underwent explorative laparotomy, and 174 (75.3\%) patients underwent surgery, including 101 (58.0\%) patients undergoing hepatic resection and 73 (42.0\%) patients undergoing pancreatic resection (see Table 3.2). Patients undergoing pancreatic resection did not significantly differ on their preoperative characteristics (age, body mass index, American Society of Anesthesiologists [ASA] grade, comorbidities, and level of physical functioning) compared to patients undergoing hepatic resection. Postoperatively, a statistically significant higher number of complications (67.1 versus $27.7 \%$; $P<0.001$ ), increased median length of hospital stay (13.0 versus 7.0 days; $\mathrm{P}<0.001)$, and increased median time to recovery of physical functioning (6.0 versus 3.0 days; $\mathrm{P}<0.001)$ were observed in patients undergoing pancreatic resection compared to patients undergoing hepatic resection. 
Multivariable logistic regression was performed for each outcome measurement (postoperative complications, delayed recovery of physical functioning [ $\geq 5$ days], prolonged length of hospital stay [ $\geq 10$ days]) based on univariable analysis (see Appendix 1, Table 3A.1). Each model included the predictor variable of perceived level of physical functioning measured with the DASI or VSAQ, and the type of resection (pancreatic resection) (see Table 3.3).

Impact of postoperative complication

Univariable analysis showed that the presence of a comorbidity, ASA score III, two performancebased parameters (relative SRT performance and 2MWT test performance), perceived level of physical functioning (VSAQ and DASI), and the type of surgery were associated with a delayed recovery of physical functioning ( $\geq 5$ days) when experiencing a complication $(P<0.05)$. Multivariable analysis yielded a model predicting delayed recovery of physical functioning when experiencing a complication with DASI (OR of 0.689; 95\% confidence interval [Cl] 0.546-0.871; $\mathrm{P}=0.002$ ) and pancreatic resection (OR of 7.817; 95\% Cl 3.510-17.408; $\mathrm{P}<0.01$ ). 


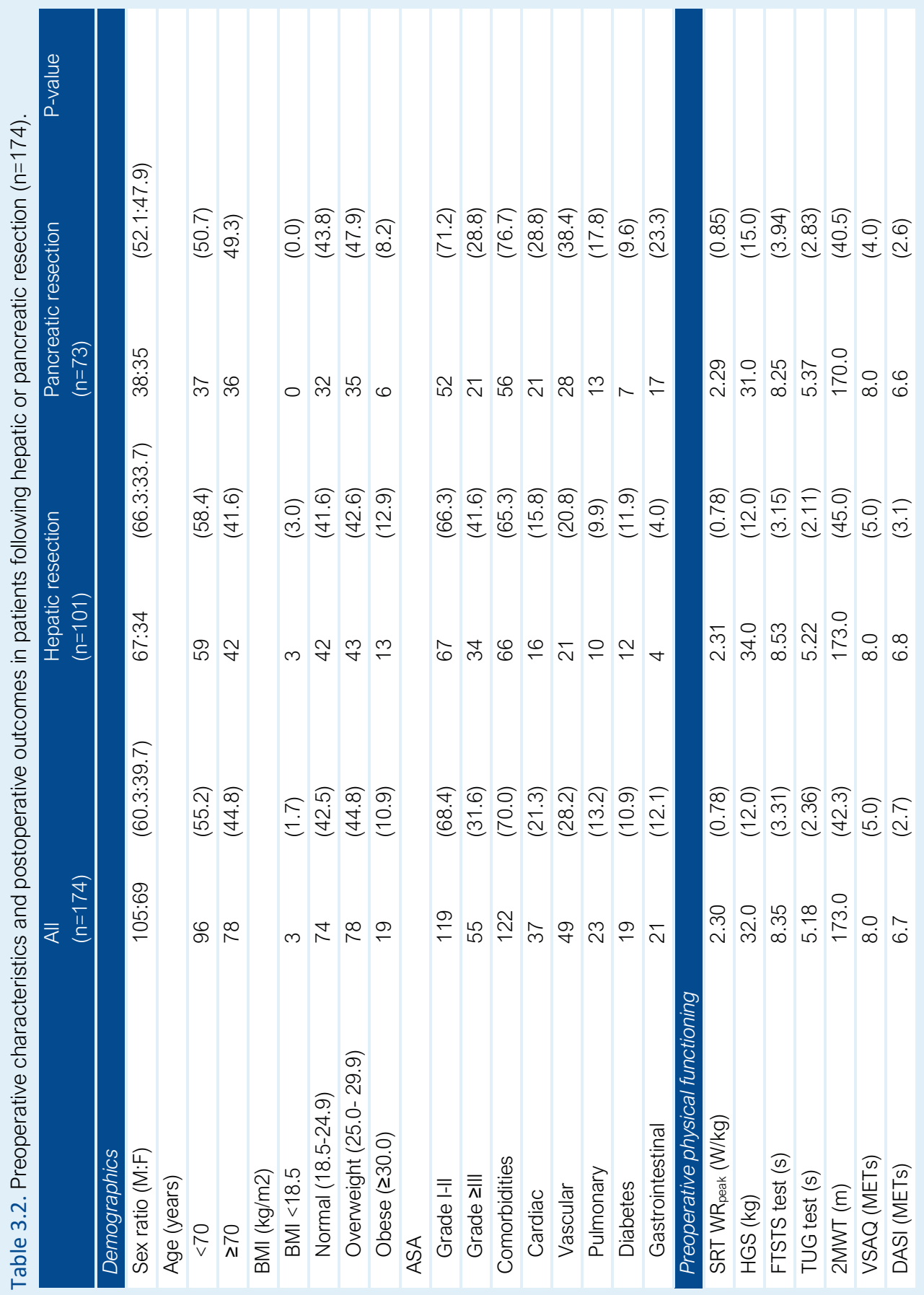




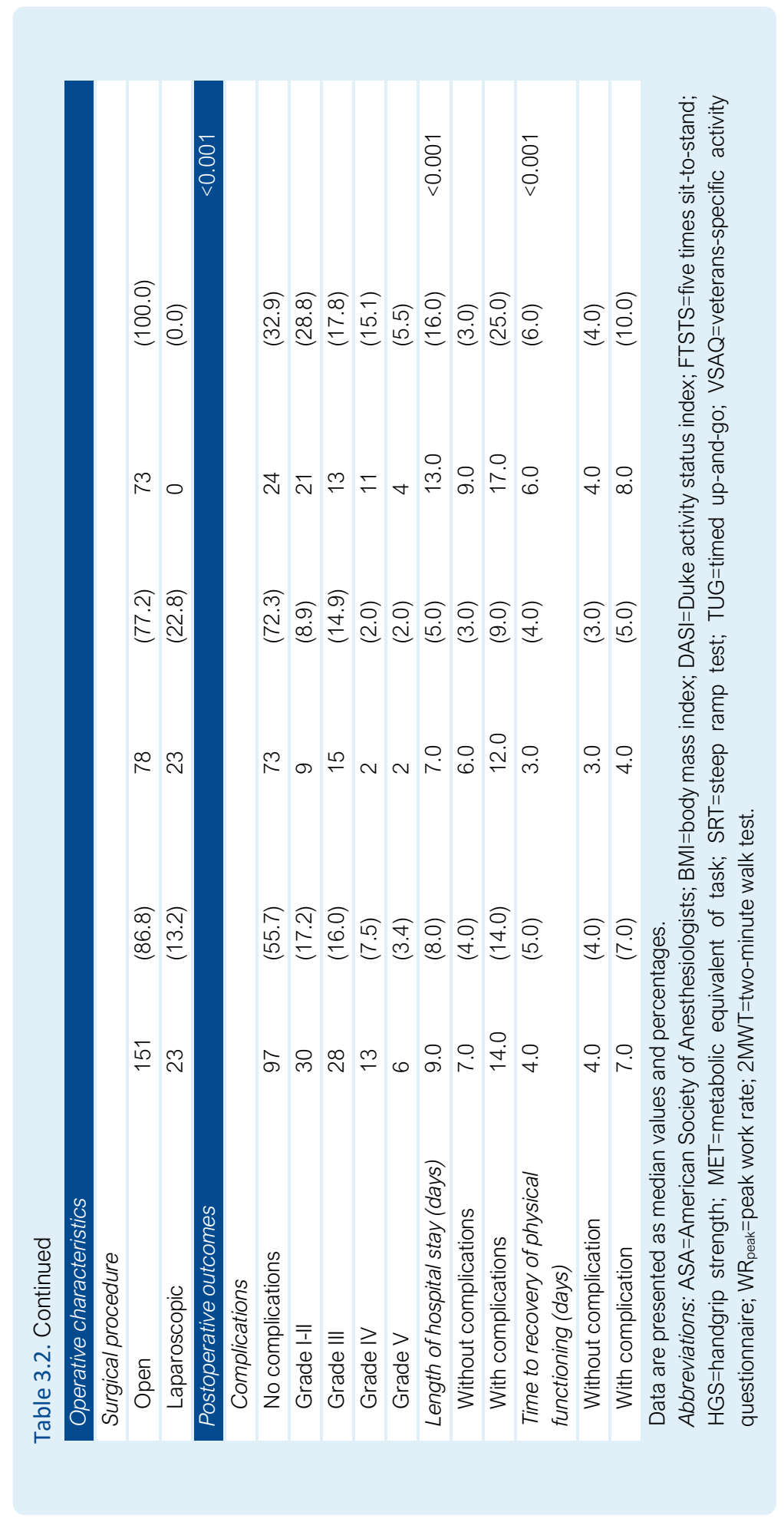




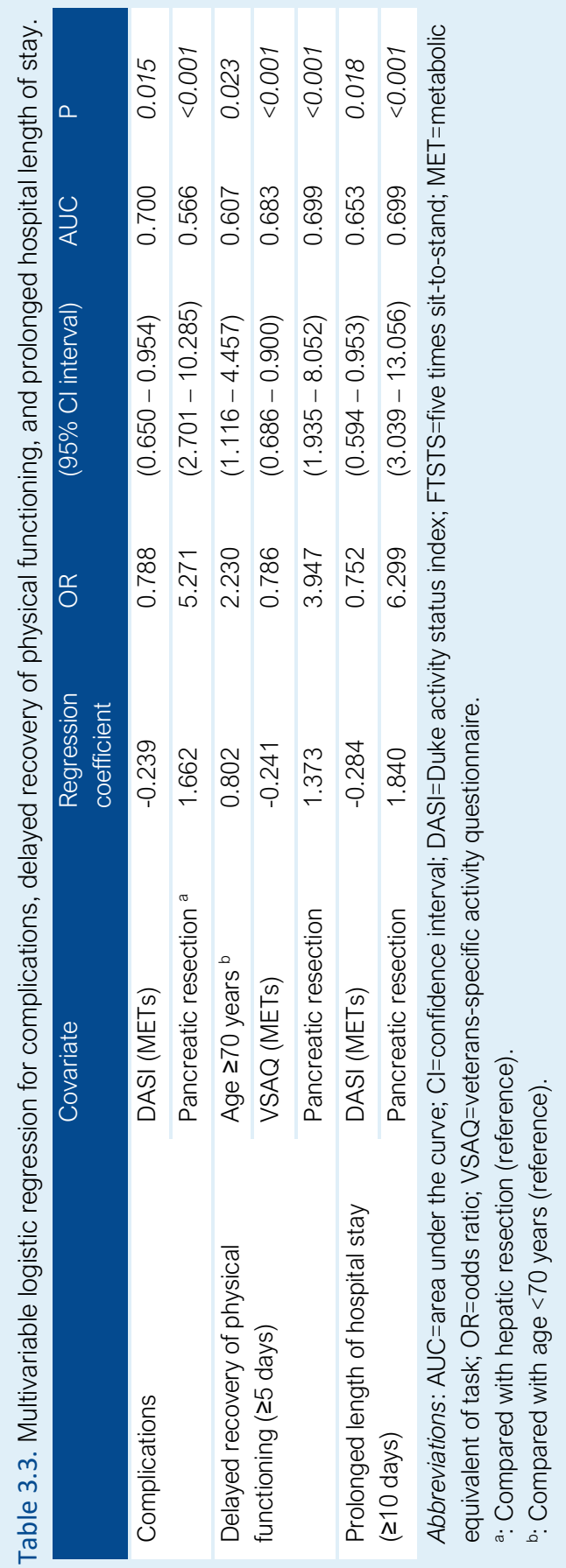




\section{DISCUSSION}

The transition experiment in routine HPB surgical and physical therapy care showed that a health care transition is dynamic and complex, demands multiple strategies, and requires interactions and alignment amongst different actors in and outside the system. Different strategies were used in a serial and in a parallel manner by the embedded scientist. These included education, time and content investment in relationships and communities, and a critical reflection on own practices by learning about optimal monitoring of a patient's pre- and postoperative physical functioning. The strategies resulted in an interaction between daily perioperative physical therapy management and scientific research, debate and reading. Knowing that perioperative physical therapy is evolving [14], this study may provide insight from a single niche on how to achieve an overall system change. Over a period of three and a half year, a collaborative interdisciplinary transition approach towards $\mathrm{P} 4$ perioperative physical therapy care resulted mainly in changes in the predictive and preventive stream. Changes in de predictive stream mainly included the development and stable use of an outpatient preoperative assessment clinic and the identification of perceived level of physical functioning, two performance based-tests, and type of resection as preoperative predictor variables for short-term (30 days) postoperative physical functioning related outcomes. In the preventive stream, a prehabilitation network of physical therapists $(n=28)$ working within the catchment area of the hospital $(30 \mathrm{~km})$ was established, six in-hospital strategies to improve physical activity amongst patients were developed, and preoperative predictor variables related to the impact of a postoperative complication were identified. The personalized stream followed as a result of the developments in the preventive and predictive streams. The associative parameters established in the predictive stream and close monitoring (titration) of a patient's physical functioning, has led to individually tailored pre- and postoperative physical therapy interventions (preventive and personalized streams). The changes in the perioperative physical therapy management with the daily monitoring of each individual patient's recovery of physical functioning by the physical therapists coincided with a decreased time to recovery of physical functioning and length of hospital stay.

The managerial degrees of freedom for the embedded scientist frontrunner (meaning time and support to employ activities) and the composition of the transition team to enable changes in clinical practice both may have had a pivotal role here, as is known for transition experiments [30]. The embedded scientist was provided with academic support and time (0.5 fte) for innovation activities. Within the process, the strength of existing habits should not be ignored when implementing new practices [31]. Frequent reminders, goal-setting, and defining the hereto 
related responsibilities with a close follow-up are important for two reasons. Firstly, to make people aware of their habits, and secondly to optimize the habits and mindset in a team towards a positive attitude when proposing changes in a system. The connection with the nurse specialist as the spider in the patient's web was essential in the knowledge co-production concerning the content, tools, and organization of the preoperative assessment. Furthermore, the outpatient preoperative assessment unit was an attractor for other health care professionals bringing in their knowledge and values. Currently, further optimization of the preoperative assessment unit is being explored to optimally map each patient's perioperative risks by interdisciplinary collaboration of health care professionals during a single hospital visit that is planned as soon as possible after the provisional decision to undergo hepatic or pancreatic resection. This cocreation across different actors in the system has been recognized as valuable in conveying societal impact via dynamic adaptive academic partnerships [30, 32].

Besides building adaptive partnerships in order to guide the transition process, close monitoring of process and outcome parameters was performed. Both the quantitative and qualitative data helped to gain insight, and the resulting debate thereafter was important to understand the dynamics while transforming perioperative physical therapy care. The dynamics of the object in the system here can be seen as a dynamic landscape with hills and valleys [33], in which the perioperative regimen acts as a ball moving in that dynamic landscape of hills and valleys (see Figure 3.8). To position and measure fluctuations of the ball and the system, quantitative data was measured. On top of these, gathered qualitative data assisted in the formulation of plausible attributions for the fluctuations in the perioperative care system. Changes initiated by the embedded scientist that act upon the perioperative care system and that are described with the qualitative data might be seen as attractors that aim to evolve the system towards P4 perioperative physical therapy. Instruments introduced for the assessment of the individual patient's preoperative level of physical functioning and physical fitness and the mILAS for monitoring postoperative recovery of physical functioning are validated instruments according to scientific literature [34, 35]. They lead to valuable and relevant patient-related data that were directly accessible for health care professionals. As there was no in- and exclusion criteria in the selection of patients, effects on postoperative time to recovery of physical functioning and length of hospital stay seem to be applicable to the general surgical population. The collected qualitative data about structure, culture, and practices were valuable in understanding the context and culture of the hospital, especially within the physical therapy department and HPB surgical ward. Combining qualitative and quantitative data enabled the embedded scientist and the team to see whether initiated interventions and its effects could be attributed to observed changes of the 
system over time. Hence, this provided room for debate and joined decisions about the follow-up actions. Hereto, it is not about when P4 perioperative physical therapy is achieved as a static and unchanging concept, but how the relative stability of the perioperative care system develops over time.

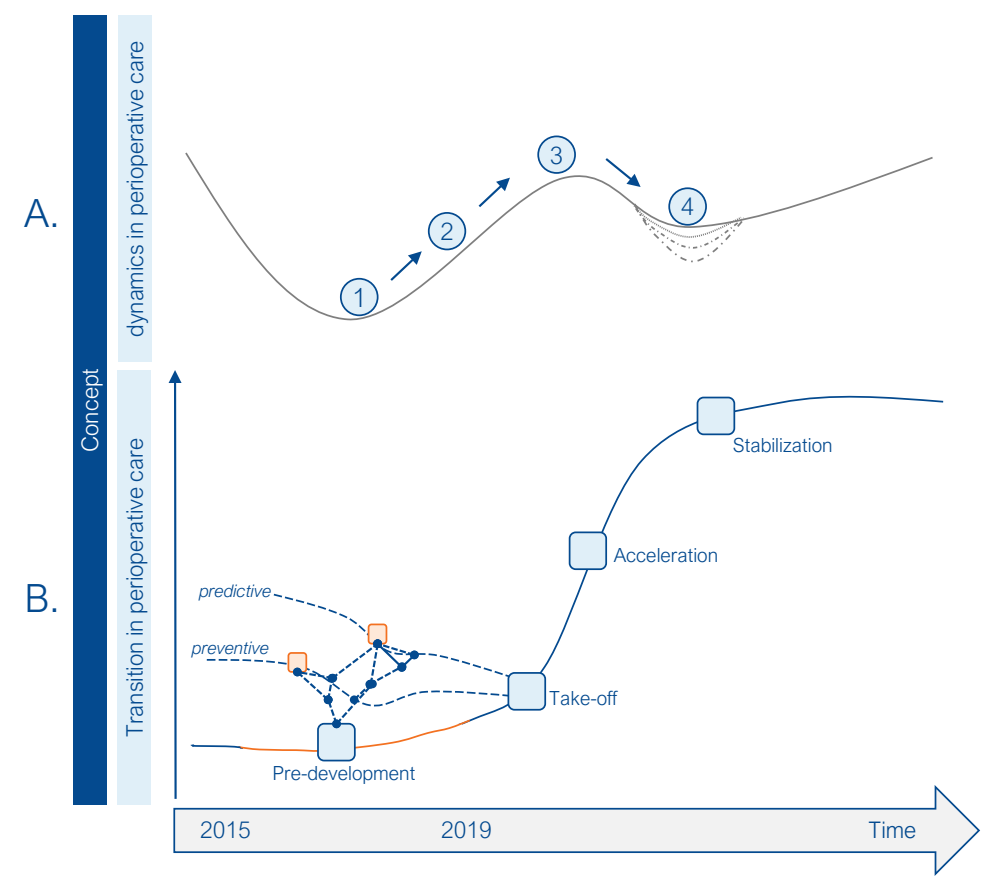

Figure 3.8. The concept consists of the dynamics of the perioperative care system and the transition in perioperative care.

A. The relatively stable perioperative care system is represented as a ball in a valley (1). Depending on the steepness of the valley, small perturbations will push the ball uphill (2). Given enough time and perturbations that are strong enough, the ball will probably be pushed on top of the hill (3). Next, the ball might fall back towards the old system when the perturbations are not strong enough (1) or might encapsulate itself in a new valley, a new and optimized (stable) state of the system (4).

B. The transition in perioperative care, which is characterized as a non-linear process divided in different phases (pre-development, take-off, acceleration, and stabilization phase) with slow and rapid changes. The perioperative care system $(\square)$ is changing due to different actors collaborating in different perioperative care streams (e.g., predictive, preventive ----) leading to the emergence of a singlesolution system $(\square)$ in the perioperative care system.

$\mathrm{A} \times \mathrm{B}$. The ball at the bottom of the steep hill requires strong attractor(s) - changes in culture, structure, and practices by different professionals - to shift it towards the top of the hill or to move it to a new stable system. Professionals in the upcoming streams (e.g., predictive, preventive) will develop specific small-system solutions contributing to the overall transition of the perioperative care system. 
Various studies have investigated the association between preoperative physical functioning and short-term postoperative outcomes in surgical populations [36-38]. Results in the current study showed that preoperative predictor variables were associated with postoperative time to recovery of physical functioning, length of hospital stay, and postoperative complications [36, 37]. The current study results are in line with studies regarding the implementation of ERAS in a multidisciplinary setting in HPB surgery on a patient's recovery of physical functioning and length of hospital stay. A systematic review and meta-analysis of four studies of the implementation of ERAS in 634 patients undergoing hepatic resection noted an accelerated recovery of physical functioning of 2.3 days and shorter length of hospital stay of 2.7 days [39]. Although these studies reported an accelerated recovery of physical functioning, this was not objectively measured but captured in the readiness for discharge criteria. In the current study, recovery of physical functioning was objectively measured by the physical therapist using the mILAS score [35]. In 108 patients undergoing total knee replacement, the introduction of a new function-tailored care pathway resulted in an accelerated recovery of physical functioning ( 0.5 day) measured with the mILAS and decreased length of hospital stay of 1.0 day [20]. Although transition experiments are context-specific in terms of culture, infrastructure, professionalism, participants, collective, and individual intentions, the transition experiment resulted in a statistically significant acceleration of recovery of physical functioning and a reduction of length of hospital stay.

There is an increasing emergence in the demand for making research relevant to patients and the wider public, thereby reducing research waste $[40,41]$. The embedded scientist approach allows for co-production (e.g., new forms of knowledge, values, and social relations) between patients and their support system, health care professionals, and researchers with the aim to narrow the gap between research and clinical practice [31, 42]. One might debate about its methodological quality when compared to the traditional randomized controlled trial (RCT) for instance, as well as about the data-intensive work. Nevertheless, this type of research involves a participatory process in which all relevant actors are collaborating to perform a research-guided transition in a real-life context, while simultaneously improving clinical practice and patient-related outcomes. A challenge with this research framework is to attribute micro-level changes in perioperative physical therapy practices towards the observed macro-level changes (time to recovery of physical functioning and length of hospital stay). The synergy of all micro-level efforts might lead to a change in the system through the cooperation of the different components. Therefore, a collaborative interdisciplinary approach is advocated to initiate and guide the transition and making research relevant to patients, the care system, and a wider public. Performing an RCT and its implementation in this respect would take more time, as the more 
artificial setting of an RCT consequently will hamper the implementation process. For example, in perioperative physical therapy management, the adoption of evidence-based physical therapy practice is low $[17,18]$, and the insights presented in this study might be helpful to accelerate the implementation of perioperative physical therapy practices elsewhere.

This study focused on the initiation and development phase of a transition experiment towards P4 perioperative physical therapy care, including the development of transition experiments, establishing connections, and creating visions and value streams within the system. Major developments were achieved in the predictive and preventive care stream followed by the personalized stream. Coinciding with the transition, a decrease in time to recovery of physical functioning and length of hospital stay was observed in patients undergoing pancreatic or hepatic resection. Further exploration, ecological validating, and creating a sustainable new perioperative physical therapy care pathway is advocated; however, the type of research design for this exploration remains challenging. 
1. Spanjersberg WR, van Sambeeck JD, Bremers A, Rosman C, van Laarhoven CJ. Systematic review and meta-analysis for laparoscopic versus open colon surgery with or without an ERAS programme. Surg Endosc. 2015;29:3443-53.

2. Counihan TC, Favuzza J. Fast track colorectal surgery. Clin Colon Rectal Surg. 2009;22:60-72.

3. Turrentine FE, Wang H, Simpson VB, Jones RS. Surgical risk factors, morbidity, and mortality in elderly patients. J Am Coll Surg. 2006;203:865-77.

4. Covinsky KE, Pierluissi E, Johnston CB. Hospitalization-associated disability: "She was probably able to ambulate, but I'm not sure". JAMA. 2011;306:1782-93.

5. Finnerty CC, Mabvuure NT, Ali A, Kozar RA, Herndon DN. The surgically induced stress response. JPEN J Parenter Enteral Nutr. 2013;37:21S-9S.

6. Massarweh NN, Legner VJ, Symons RG, McCormick WC, Flum DR. Impact of advancing age on abdominal surgical outcomes. Arch Surg. 2009;144:1108-14.

7. Hulzebos EH, van Meeteren NL. Making the elderly fit for surgery. Br J Surg. 2016;103:463.

8. Hulzebos EH, van Meeteren NL. Making the elderly fit for surgery. Br J Surg. 2016;103:e12-5.

9. Covinsky KE, Palmer RM, Fortinsky RH, Counsell SR, Stewart AL, Kresevic D, et al. Loss of independence in activities of daily living in older adults hospitalized with medical illnesses: increased vulnerability with age. J Am Geriatr Soc. 2003;51:4518.

10. Lawrence VA, Hazuda HP, Cornell JE, Pederson T, Bradshaw PT, Mulrow CD, Page CP. Functional independence after major abdominal surgery in the elderly. J Am Coll Surg. 2004;199:762-72.

11. Dutch Institute for Clinical Auditing (DICA) Leiden. DICA jaarrapportage 2017; DPCA.2017. Accessed November 2018, htttp://dica.nl/jaarrapportage-2017.
12. Junejo MA, Mason JM, Sheen AJ, Bryan A, Moore J, Foster P, et al. Cardiopulmonary exercise testing for preoperative risk assessment before pancreaticoduodenectomy for cancer. Ann Surg Oncol. 2014;21:1929-36.

13. Hood L, Flores M. A personal view on systems medicine and the emergence of proactive P4 medicine: predictive, preventive, personalized and participatory. N Biotechnol. 2012;29:613-24.

14. Bongers BC, Punt IM, van Meeteren NL. On "Prehabilitation: The emperor's new clothes or a new arena for physical therapists?" Lundberg M, Archer KR, Larsson C, Rydwik E. Phys Ther. 2018;12:127-130. Phys Ther. 2019;99:953-4.

15. Hoogeboom TJ, Dronkers JJ, Hulzebos $\mathrm{EH}$, van Meeteren NL. Merits of exercise therapy before and after major surgery. Curr Opin Anaesthesiol. 2014;27:161-6.

16. Dijstelbloem $H$, Huisman $F$, Miedema F, Mijnhardt W. Waarom de wetenschap niet werkt zoals het moet en wat daar aan te doen is. Position paper. 2013, Science in Transition. p. 31.

17. van Beijsterveld CA, Heldens AF, Bongers $B C$, van Meeteren NL. Variation in Preoperative and postoperative physical therapist management of patients opting for elective abdominal surgery. Phys Ther. 2019;99:1291-303.

18. Janssen ERC, Scheijen EEM, van Meeteren NLU, de Bie RA, Lenssen AF, Willems PC, Hoogeboom TJ. Determining clinical practice of expert physiotherapy for patients undergoing lumbar spinal fusion: a cross-sectional survey study. Eur Spine J. 2016;25:1533-41.

19. Macleod MR, Michie S, Roberts I, Dirnagl U, Chalmers I, loannidis JP, et al. Biomedical research: increasing value, reducing waste. Lancet. 2014;383:101-4. 
20. van der Sluis G, Goldbohm RA, Bimmel R, Garre FG, Elings J, Hoogeboom TJ, et al. What augmented physical activity and empowerment can bring to patients receiving total knee replacement: content, implementation, and comparative effectiveness of a new function-tailored care pathway in a routine care setting. Biomed Res Int. 2015;2015:745864

21. Grol R, Grimshaw J. From best evidence to best practice: effective implementation of change in patients' care. Lancet. 2003;362:1225-30.

22. Craig P, Cooper C, Gunnell D, Haw S, Lawson K, Macintyre S, et al. Using natural experiments to evaluate population health interventions: new Medical Research Council guidance. J Epidemiol Community Health. 2012;66:1182-6.

23. Rotmans J, Kemp R, Van Asselt M. More evolution than revolution: transition management in public policy. Foresight 2001;3:15-31.

24. de Haan FJ, Rotmans J. A proposed theoretical framework for actors in transformative change. Technological Forecasting and Social Change. 2018;128:275-286.

25. Ljungqvist $\mathrm{O}$, Scott $\mathrm{M}$, Fearon $\mathrm{KC}$. Enhanced recovery after surgery: a review. JAMA Surg. 2017;152:292-8.

26. Dunne DF, Jones RP, Lythgoe DT, Pilkington FJ, Palmer DH, Malik HZ, et al. Cardiopulmonary exercise testing before liver surgery. J Surg Oncol. 2014;110:43944.

27. Junejo MA, Mason JM, Sheen AJ, Moore J, Foster P, Atkinson D, et al. Cardiopulmonary exercise testing for preoperative risk assessment before hepatic resection. Br J Surg. 2012;99:1097-104.

28. Fleuren MA, Vrijkotte $S$, Jans MP, Pin R, van Hespen A, van Meeteren NL, et al. The implementation of the functional task exercise programme for elderly people living at home. BMC Musculoskelet Disord. 2012;13:128.

29. Glasziou P, Irwig L, Mant D. Monitoring in chronic disease: a rational approach. BMJ. 2005;330:644-8.
30. Loorbach $\mathrm{D}$, Rotmans J. The practice of transition management: Examples and lessons from four distinct cases. Futures. 2010;42:237-46.

31. Vindrola-Padros $\mathrm{C}$, Eyre L, Baxter $\mathrm{H}$, Cramer H, George B, Wye L, et al. Addressing the challenges of knowledge co-production in quality improvement: learning from the implementation of the researcher-in-residence model. BMJ Qual Saf. 2019;28:67-73.

32. Greenhalgh T, Jackson C, Shaw S, Janamian T. Achieving research impact through co-creation in community-based health services: literature review and case Study. Milbank Q. 2016;94:392-429.

33. Thelen E, Smith B. "Dynamic systems theories". Handbook of child psychology 1 (2007).

34. Van Beijsterveld CA, Bongers BC, Den Dulk M, Van Kuijk SMJ, Dejong CH, Van Meeteren NL. The association between preoperative physical functioning and short-term postoperative outcomes: a cohort study of patients undergoing elective hepatic resection. HPB (oxford). 2019;21:1362-70.

35. Elings J, Zoethout S, Ten Klooster PM, van der Sluis G, van Gaalen SM, van Meeteren $N L U$, et al. Advocacy for use of the modified lowa Level of Assistance Scale for clinical use in patients after hip replacement: an observational study. Physiotherapy. 2019;105:108-13.

36. Dronkers JJ, Chorus AM, van Meeteren NL, Hopman-Rock M. The association of pre-operative physical fitness and physical activity with outcome after scheduled major abdominal surgery. Anaesthesia. 2013;68:67-73.

37. Heldens AFJM, Bongers BC, Lenssen AF, Stassen LPS, Buhre WF, van Meeteren NLU. The association between performance parameters of physical fitness and postoperative outcomes in patients undergoing colorectal surgery: An evaluation of care data. Eur J Surg Oncol. 2017;43:2084-92. 
38. Elings J, Hoogeboom TJ, van der Sluis G, van Meeteren NL. What preoperative patient-related factors predict inpatient recovery of physical functioning and length of stay after total hip arthroplasty? A systematic review. Clin Rehabil. 2015;29:477-92.

39. Song W, Wang K, Zhang RJ, Dai QX, Zou $\mathrm{SB}$. The enhanced recovery after surgery (ERAS) program in liver surgery: a metaanalysis of randomized controlled trials. Springerplus. 2016;5:207.
40. Crowe S, Giles C. Making patient relevant clinical research a reality. BMJ.2016;355:i6627.

41. Moher D, Glasziou P, Chalmers I, Nasser M, Bossuyt PMM, Korevaar DA, et al. Increasing value and reducing waste in biomedical research: who's listening? Lancet. 2016;387:1573-86.

42. Filipe A, Renedo A, Marston C. The coproduction of what? Knowledge, values, and social relations in health care. PLoS Biol. 2017;15:e2001403.

\section{NOTES}




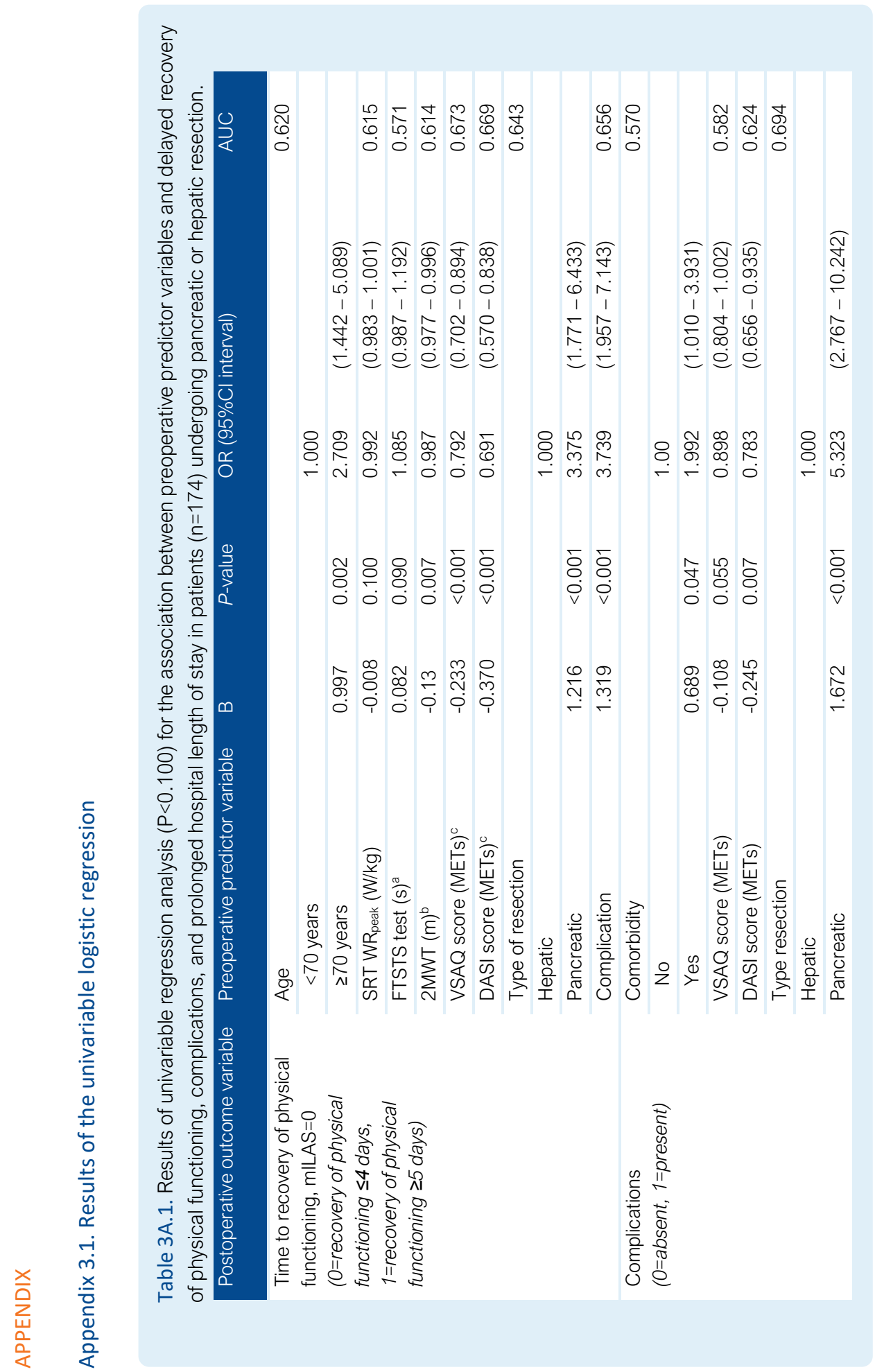




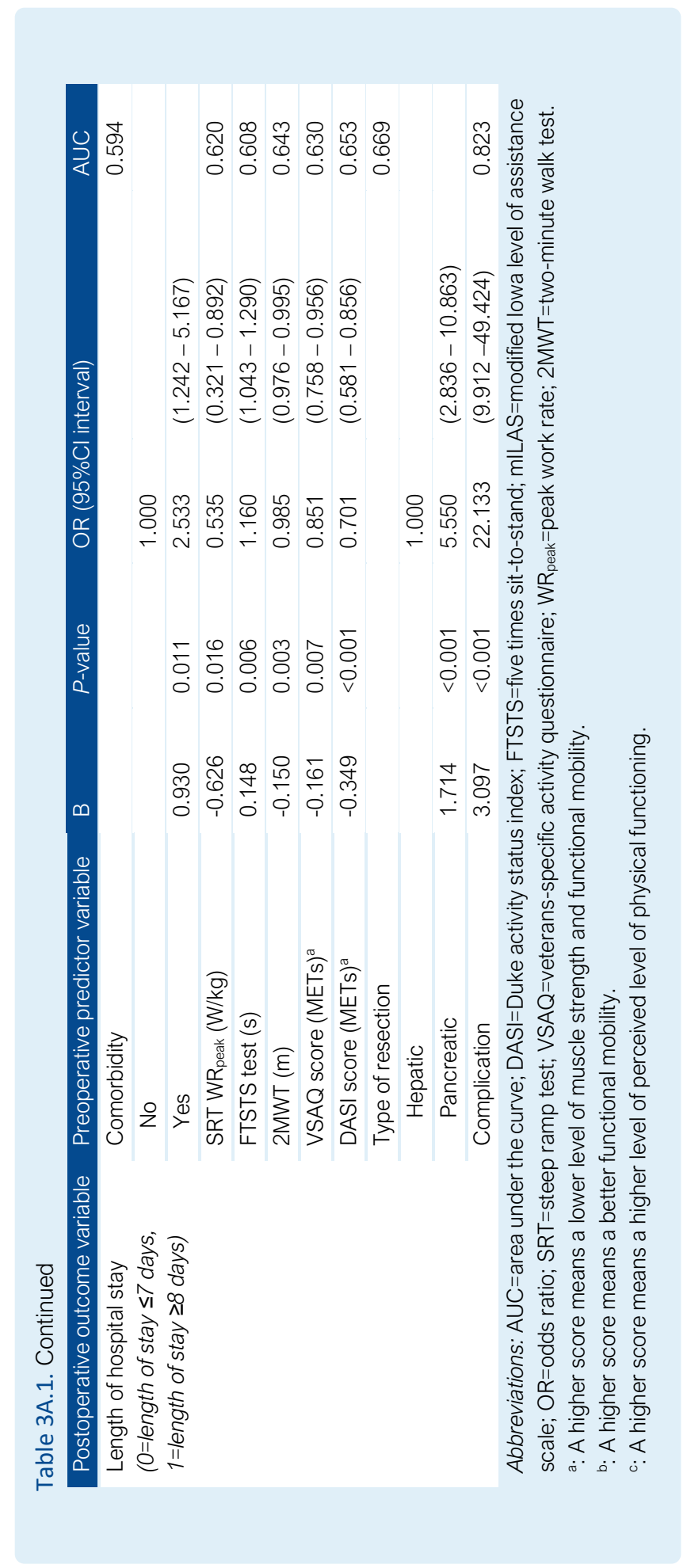



Performance tests voor het evalueren van het fysiek functioneren zijn essentieel in de bes/uitvorming tussen patiënt, mantel en zorgverleners aangaande preoperatieve zorginterventies bij grote abdominale chirurgie.




\section{Chapter 4}

The association between preoperative physical

functioning and short-term postoperative outcomes: a cohort study of patients undergoing elective hepatic resection

Christel A. van Beijsterveld | Bart C. Bongers | Marcel den Dulk | Sander M. van Kuijk | Cornelius H. Dejong | Nico L. van Meeteren 


\section{ABSTRACT}

\section{Background}

This study evaluated the association between practical performance-based indices of preoperative physical functioning and short-term postoperative outcomes in patients undergoing hepatic resection.

\section{Method}

Preoperative characteristics and results of practical performance-based tests of physical functioning were analyzed concerning the effect on postoperative outcomes (recovery of physical functioning, non-surgical complications, and length of hospital stay) using univariable and multivariable logistic regression.

\section{Results}

Perioperative data of 96 patients showed that besides the conventional risk-factors (American Society of Anesthesiologists grade III and BMI), lower absolute steep ramp test performance (in watts; OR 0.992), and lower perceived level of functional capacity to perform activities of daily life (ADL) on Duke activity status index (in metabolic equivalent of task (MET); OR 0.806) and lower score on the veterans-specific activity questionnaire (in MET, OR 0.875) were associated with delayed recovery of physical functioning. Furthermore, more comorbidities, worse functional mobility, and lower levels of perceived functional capacity to perform ADL were associated with non-surgical complications and length of hospital stay.

\section{Conclusion}

Adequate preoperative performance and perceived level of functional capacity to perform ADL appear to be of importance to identify individual patients that are at risk of a complicated postoperative course. 


\section{INTRODUCTION}

Hepatic resection is the primary modality of treatment in patients diagnosed with benign and malign hepatic tumors [1]. Despite advances in diagnostic, surgical, and anesthetic techniques, as well as improvements in perioperative care management due to enhanced recovery after surgery (ERAS) [2] and fast-track programs [3], hepatic resection remains an operation with a high risk of morbidity and mortality. In the Netherlands, morbidity rate ranges from $8.9 \%$ to $22 \%$, whereas mortality ranges from $1.0 \%$ to $5.0 \%$, which is dependent on the type of the surgery and the extent of the hepatic resection [4]. Besides advances in perioperative care management, it remains to be seen what type of patient-related factors can be improved in order to lower the risk of inappropriate postoperative outcomes. For instance, the patient's physical functioning level prior to elective major abdominal surgery is known to be associated with postoperative morbidity and mortality in patients undergoing colorectal resection $[5,6]$.

Preoperative aerobic capacity measured by cardiopulmonary exercise testing (CPET) has been reported to be a valuable non-invasive performance test to predict part of the risk for postoperative morbidity, mortality, and length of stay in patients undergoing hepatic resection [712]. However, its use requires sophisticated equipment and trained staff. Hence, to use practical performance tests to evaluate a patient's preoperative physical functioning level prior to surgery should be considered. Practical performance-based tests such as the timed up-and-go (TUG) test, stair-climb test, incremental shuttle walk test, and six-minute walk test (6MWT) have been identified as feasible and relatively accurate in predicting postoperative complications in patients undergoing all sorts of surgery [13-15], including elective abdominal surgery [16-18].

Consequently, the added value of the use of practical performance-based tests in the preoperative risk assessment should become clear as a prerequisite for clinical reasoning and shared decision-making concerning the choice and use of prophylactic, surgical and anesthetic interventions. By doing so, their risk for perioperative morbidity and mortality might be decrease [19-12]. In this study we describe pre- and postoperative functioning in patients undergoing elective hepatic resection by evaluating the role of preoperative practical performance-based tests and its association with short-term (30-day) postoperative outcomes (time to recovery of physical functioning, non-surgical complications, and length of hospital stay). 


\section{METHODS}

This was a single-center prospective cohort study in patients undergoing hepatic resection for benign or malignant tumors at the Maastricht University Medical Center (Maastricht UMC+), Maastricht, the Netherlands. Patients with benign or malignant pathology with an American Society of Anesthesiologists (ASA) score of I-IV undergoing hepatic resection for benign and malignant tumors were monitored pre- and postoperatively on their physical functioning as part of usual care. All consecutive patients with no objection for using their usual care data for research purposes were included. When essential data about pre-and postoperative outcomes was missing, the data from that patient was excluded from analysis. The data collection was performed between March 2016 and December 2017. Baseline patient characteristics included age, sex, body mass index (BMI, in $\mathrm{kg} / \mathrm{m}^{2}$ ), ASA score, Charlson comorbidity index (CCl), surgical approach (laparoscopic or open), and type of liver resection (e.g., (extended) hemihepatectomy, segmental resection, wedge resection). The medical ethical committee of the Maastricht UMC+ decided (15-4-234) that this study met the ethical policies of the Maastricht UMC+ and the regulations of the Dutch government.

\section{Usual care pathway}

As part of the preoperative multidisciplinary risk screening, patients scheduled for hepatic resection for benign and malignant tumors were referred by the hepato-pancreato-biliary surgeon or nurse practitioner to a physical therapist at the outpatient physical therapy department, approximately three weeks prior to surgery. The physical therapist screened the patient for their level of physical functioning. Additionally, patients received an information leaflet about the content and expectations of the postoperative physical therapy treatment during hospitalization and were educated regarding the importance of physical activity pre- and postoperatively. All patients were routinely admitted one day prior to surgery to the HPB ward. Intraoperatively, patients received a thoracic epidural for analgesia. Approximately 72 hours post-surgery, the thoracic epidural was removed. Postoperatively, patients were transferred to the HPB ward as soon as possible". After surgery, all patients received postoperative physical therapy once a day, starting the first day after surgery (postoperative day 1). Physical therapy consisted of airway clearing exercises, practicing transfers, walking, and other functional abilities based on the individual patient needs and progress. The recovery of physical functioning was monitored by the physical therapist using the modified lowa levels of assistance scale (mILAS) [22]. 
Preoperative performance and perception of physical functioning

All patients were screened by an experienced physical therapist with help of a protocolled test battery on aerobic capacity, muscle strength, functional mobility, and perceived level of functional capacity to perform activities of daily life (ADL). Prior to the preoperative assessment, the physical activity readiness questionnaire [23] was filled out by the patient under supervision of the physical therapist as a simple screening tool to evaluate whether there were contra-indications for exercise testing.

\section{Aerobic capacity}

The steep ramp test (SRT), a short-time maximal exercise test performed on a cycle ergometer [24], was used to estimate the patient's aerobic capacity. A previous study in adult cancer survivors demonstrated a strong correlation between the primary outcome measure of the SRT, the attained peak work rate ( $\left(\mathrm{R}_{\text {peak }}\right)$, and the peak oxygen uptake $\left(\mathrm{V}_{2 \text { peak }}\right)$ achieved during CPET ( $r$ of 0.82) [25]. This suggests that the SRT is a valid test to estimate aerobic capacity at the group level. De Backer et al. also showed that the SRT is a reliable test (intra-class correlation coefficient [ICC] for the achieved WR peak of 0.996) [25]. For each patient, seat height was adjusted to a comfortable leg length. During the test, heart rate was monitored by using an elastic belt with a heart rate sensor (Polar T31i transmitter, Polar, Kempele, Finland). To make the SRT suitable for our surgical population, the original protocol (work rate increments of $25 \mathrm{~W}$ each $10 \mathrm{~s}$ [26]) was modified. After a two-minute warm-up of unloaded cycling, the test started by applying resistance on a calibrated cycle ergometer (Lode Corival Rehab, Lode BV, Groningen, the Netherlands) with increments of $10 \mathrm{~W}$ every $10 \mathrm{~s}$ in a ramp like manner $(1 \mathrm{~W} / \mathrm{s})$. The patient was instructed to maintain a pedaling frequency around 80 rotations $/ \mathrm{min}$. The protocol continued until there was a sustained drop in the patient's pedaling frequency below 60 rotations/min despite strong verbal encouragement. The main outcome measure of the SRT was the attained WR peak, the point at which the patient's pedaling frequency definitely dropped $<60$ rotations/min. Efforts were considered to be maximal when the patient showed subjective signs of intense effort (e.g., unsteady biking, sweating, facial flushing, and clear unwillingness to continue despite encouragement).

\section{Muscle strength}

Muscle strength was evaluated using the five times sit-to-stand (FTSTS) test and by measuring the patient's handgrip strength (HGS). The FTSTS test is commonly used to measure lower extremity muscle strength and functional mobility [27-28]. The patient was instructed to start the 
test from a sitting position on a chair (43 to $47 \mathrm{~cm}$ in height) with the arms folded across the chest and the feet placed comfortably on the floor (knees flexed $90^{\circ}$ ). From this position, the physical therapist encouraged the patient to stand up from the chair to a fully erected position and to sit down again, five times, as quickly as possible. The time, ending with timing after the fifth stand, required for the five repetitions of rising from a chair and sitting was timed with a stopwatch (two decimals). The test was performed three times, with 30s rest in between, and the best performance in seconds was used. The FTSTS test is easy to perform in clinical practice and has been reported to be a reliable method to assess test-re-test reliability (ICC of 0.89) in communitydwelling elderly [29].

HGS of the dominant hand was measured to estimate the patient's general muscle strength by using a hand-held dynamometer (JAMAR $®$ Hydraulic Hand Dynamometer, JAMAR, Patterson Medical Holdings, Inc., Illinois, USA). The patient was seated in a chair (43 to $47 \mathrm{~cm}$ in height) with the shoulders adducted, the elbow flexed at $90^{\circ}$, and the forearm in neutral position without any support from the chair. The handle of the dynamometer was placed in such a position that the middle phalanges had to rest on the handle. The patient was asked to squeeze the dynamometer to maximum ability with encouragement of the physical therapist. The maximal grip score $(\mathrm{kg})$ of the three attempts (with $30 \mathrm{~s}$ of rest in between) was recorded. Handgrip strength measured with the Jamar dynamometer has been shown to have an adequate intra- and interrater reliability (ICC values from 0.85 to 0.98 ) in healthy adults [30].

\section{Functional mobility}

The two-minute walk test (2MWT) and the TUG test were used to measure functional mobility [31]. The 2MWT measures the functional walking capacity and was performed in a corridor of the physical therapy department over a length of $15 \mathrm{~m}$. Patients were instructed to walk as far as they could in two minutes using their customary walking aid, if applicable. During the test, the physical therapist encouraged the patient after one-minute elapse by saying "you are doing well, you have one-minute left". The distance walked (in $\mathrm{m}$ ) was recorded. The test has an adequate test-retest reliability (ICC of 0.82) in community-dwelling healthy adults (18-85 years) [32].

The TUG test measures basic functional mobility and reflects the ability to rise from an arm chair (43 to $47 \mathrm{~cm}$ in height), walk a short distance $(3 \mathrm{~m})$, return to the chair, and sit down again, all as quick as possible [33]. The patient has three attempts, with $30 \mathrm{~s}$ of rest between attempts. The physical therapist recorded the time of each attempt and the best attempt was used for analysis. 
The inter-rater (ICC of 0.99) and intra-rater (ICC of 0.99) reliability of the TUG test has been reported to be excellent in geriatric patients [33].

\section{Perceived level of functional capacity to perform $A D L$}

To gain insight in the patient's perceived level of functional capacity to perform ADL, each patient filled out two questionnaires with help of the physical therapist: the veterans-specific activity questionnaire (VSAQ) and the Duke activity status index (DASI). The VSAQ consists of a list of activities presented in a progressive order and linked to a particular metabolic equivalent of task (MET) score, with a maximum of 13 METs. Patients were asked to determine which kind of activities of daily life they were able to perform routinely without experiencing symptoms or with only minimal symptoms. A line is drawn below those activities and the corresponding MET score is noted. The VSAQ has a good intra- (ICC of 0.882) and inter-rater reliability (ICC of 0.904), measured in healthy older adults, to assess the patient's performance of activities of daily life [34].

The DASI is a self-administered twelve-item questionnaire measuring the ability to perform a common set of activities of daily life and recreational activities to assess functional capacity [35]. Each item of the questionnaire has a corresponding weight. The total score of the DASI consist of the weights for each 'yes' response. The DASI score correlated well with aerobic capacity $\left(V_{O_{2 p e a k}}\right.$ Spearman correlation coefficient of 0.81$)$ and a rough estimation of a patients $V_{0}$ 2peak can be made by the formula: $V_{\text {2peak }}=($ DASI Score $\times 4.6)+9.2$ [35]. Dividing the estimated $V_{\text {2peak }}$ by 3.5 ( 1 MET equals an oxygen uptake of $3.5 \mathrm{~mL} / \mathrm{kg} / \mathrm{min}$ ), an estimation of the patient's peak MET score (intensity of physical activities) can be made.

\section{Postoperative outcomes}

The primary postoperative outcome measure was time to recovery of physical functioning (measured by mILAS) [22]. Time to recovery of physical functioning was defined as the time between the day of surgery and day at which the patient reached a mILAS score of 0 (in days). Secondary postoperative outcome measures were 30-day morbidity (non-surgical complications), and length of hospital stay (in days). Complications were categorized recorded by Clavien-Dindo classification and were divided in surgical (defined as wound infection, bile leak, hemorrhage, liver failure, abdominal abscess, and pleura effusion) and non-surgical complications (defined as pneumonia, cardiac, and thrombo-embolic complications). 


\section{Statistical analysis}

The Statistical Package for the Social Sciences for Windows (version 23.0; IBM, SPSS Inc., Chicago, IL, USA) was used for statistical analysis. For all continuous variables, normality was tested with the Kolmogorov-Smirnov test. The association between the independent variables and dependent variables was assessed using univariable logistic regression. We chose a less stringent alpha for pre-selection of variables of 0.20 instead of 0.05 to prevent too early deletion of variables from the model, as suggested by prediction modeling guidelines [36]. As a result, $95 \%$ confidence intervals of the estimated odds ratios (ORs) may contain 1.0. However, this is a proven strategy to explore univariate associations between potential predictor variables and outcome variables. Dependent variables were dichotomized according to median time to recovery of physical functioning ( $0=$ recovery of physical functioning $\leq 3$ days, $1=$ recovery of physical functioning $\geq 4$ days), development of non-surgical complications ( $0=$ =absent, $1=$ =present), and median prolonged length of hospital stay ( $0=$ hospital length of stay $\leq 7$ days, $1=$ hospital length of stay $\geq 8$ days). Multivariable logistic regression with backward stepwise elimination was used to determine the strongest independent predictors for the dependent variables with ten events per independent variable. The selection of the predictors was based on the literature, expert opinion, and results from univariable logistic regression $(P$-value of $<0.200)$. A combination of conventional predictors and physical functioning predictors was chosen. Correlations between each two variables were checked and if they were $<0.70$ we chose to include only the variable we considered is easiest to measure. The Akaike Information Criterion was used as stopping rule for the backward stepwise elimination, which is equivalent to using an alpha of 0.157 for variables of 1 degree-of-freedom, as per prediction modeling guidelines [37]. Receiver operating characteristic (ROC) curve analysis was performed to evaluate the diagnostic ability of the tests to discriminate between the two patient states. 


\section{RESULTS}

In total 125 patients were identified as potential candidate for the study. Twenty-nine patients (23.2\%) were excluded for analysis because they received other treatments for their hepatic malignancies instead of hepatic resection or did not receive a preoperative screening. Hence, we eventually analyzed data from 96 patients (76.8\%) undergoing hepatic resection for benign or malign tumors that received pre- and postoperative physical therapy. In eight patients (8.3\%), data from the preoperative screening were not complete, as data from the SRT were lacking (not available because of logistic problems $(n=1,12.5 \%)$, contra-indications (cardiac $(n=2)$ and neurological $(n=1)$ based on the PAR-Q $(n=3,37.5 \%))$, personal reasons of the patient $(n=3$, $37.5 \%)$, and software problems during the test $(n=1,12.5 \%))$. The flow-chart is presented in Figure 4.1. No adverse events occurred during the preoperative screening and no adverse events were reported by patients later on. Postoperative recovery of physical functioning (mILAS scores) data of ten patients (10.4\%) were missing because of logistic problems. In Figure 4.2, the preoperative characteristics and performance parameters of physical functioning and postoperative outcomes are presented. Two patients (2.1\%) died after surgery. One patient died nine days after surgery because of systemic inflammatory response syndrome leading to multiorgan failure. The other patient was discharged after nine days in a good physical status but was readmitted after three days because of an abscess with abdominal wound dehiscence. He died during his second surgery because of cardiac arrest. 


\section{Flowchart}

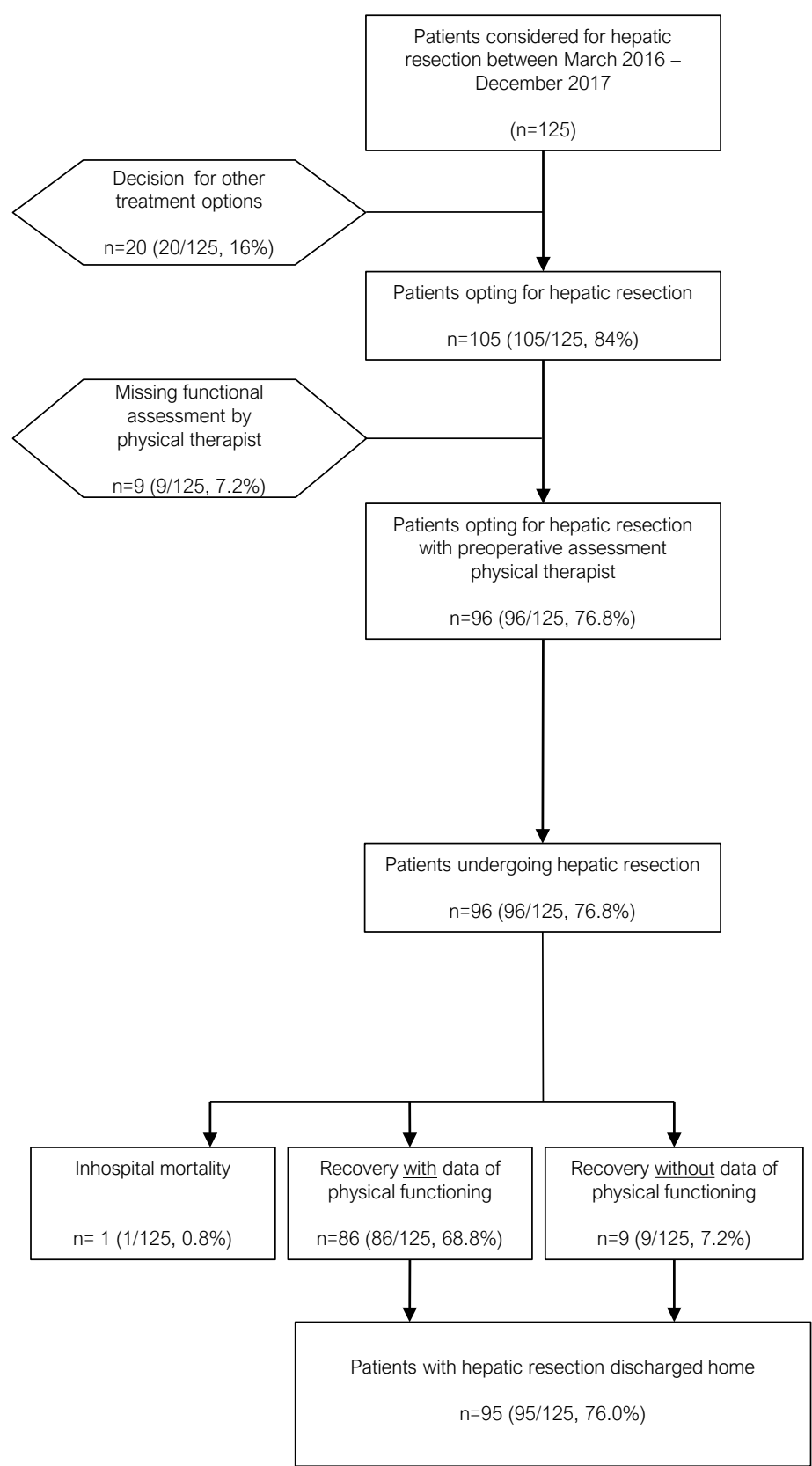

Figure 4.1. Flow chart of study population selection. 
Time to recovery of physical functioning $(n=86)$

The median \pm IQR time to recovery of physical functioning (mILAS score of 0 ) was $3.0 \pm 3.0$ days. When data were clustered according to surgical approach (open approach ( $n=68,79.1 \%$ ) versus laparoscopic approach $(n=18,20.9 \%))$, a statistically significant difference in time to recovery of physical functioning ( $4.0 \pm 3.0$ versus $2.0 \pm 1.0$ days; $P=0.003$ ) was observed (Figure 4.2, Box 2). Early recovery of physical functioning (recovery of physical functioning $\leq 3$ days) was present in 49 patients (57.0\%) and delayed recovery of physical functioning (recovery of physical functioning $\geq 4$ days) was present in 37 patients (42.0\%). A BMl between $25-30 \mathrm{~kg} / \mathrm{m}^{2}$, ASA score III, aerobic capacity (absolute SRT performance), and preoperative perceived level of functional capacity to perform ADL (VSAQ and DASI) all showed an univariate association $(P<0.200)$ with time to recovery of physical functioning. Results of the univariable regression analysis are shown in Table 4.1. For aerobic capacity measured with the SRT, the odds ratio (OR) was 0.992 per watt (OR 0.818 per 45 watt). The area under the curve (AUC) was 0.616 (95\% $\mathrm{Cl} 0.491$ to 0.740 , $P=0.076)$. With a one-MET difference at the VSAQ, the OR of a delayed recovery of physical functioning was 0.875 . For a patient with an ASA score III, the OR of a delayed recovery of physical functioning was 2.349 compared with patients with an ASA score I-II. Multivariable logistic regression with stepwise elimination starting with four variables, two conventional (ASA and $\mathrm{BMI}$ ) and two physical functioning parameters (absolute SRT performance and HGS) yielded a model including two variables $(P<0.157)$, ASA score III vs I-II (OR of 2.405 ; $95 \% \mathrm{Cl}$ of 0.901 to 6.420; $P=0.080$ ) and absolute SRT performance (OR of $0.993 ; 95 \% \mathrm{Cl} 0.984$ to $1.002 ; P=0.144$ ). Patients with an ASA score III and a lower absolute WR $\mathrm{Weak}_{\text {pe }}$ (in W) at the SRT were more likely to have a prolonged recovery of physical functioning. 

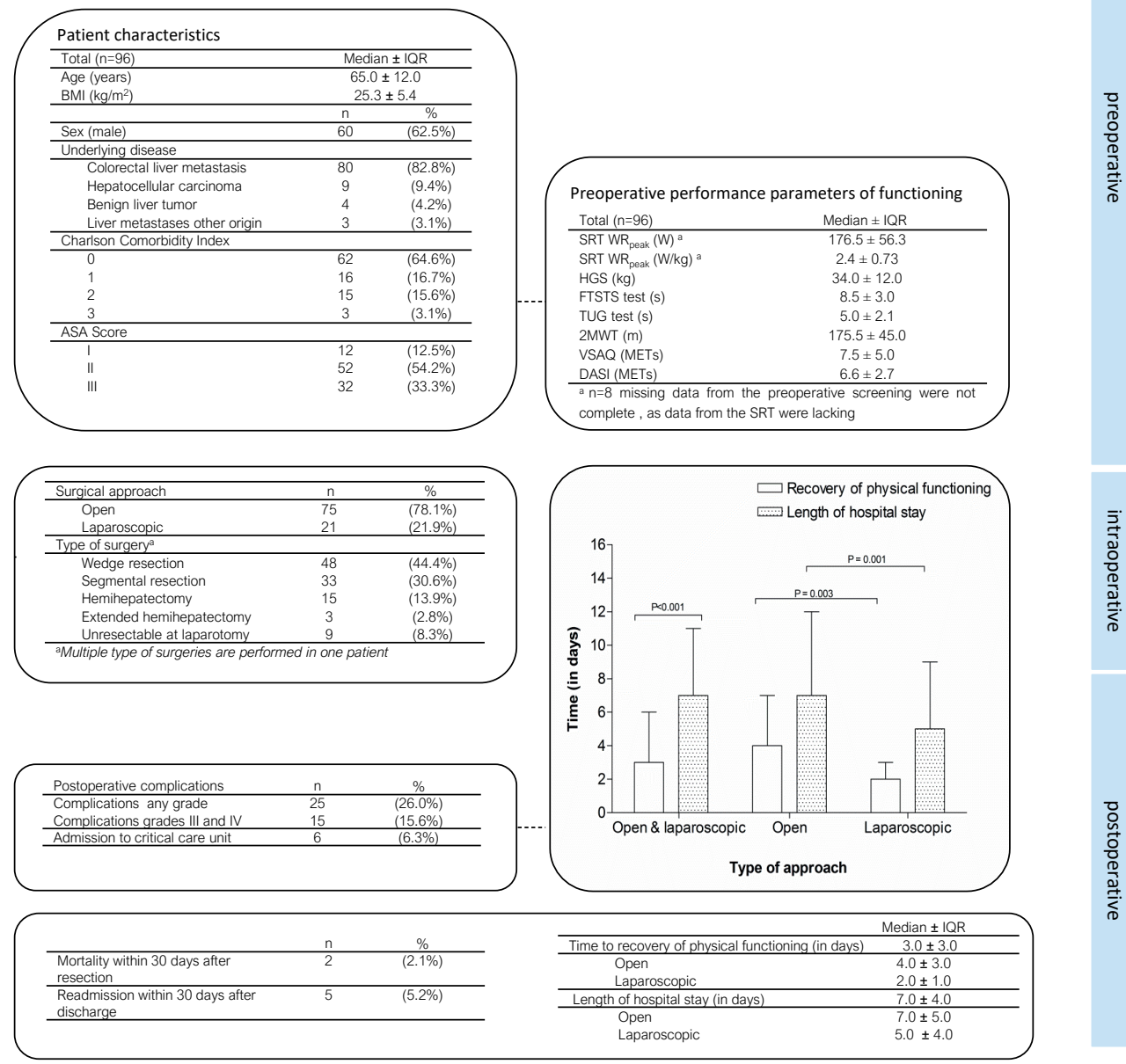

Figure 4.2. Preoperative characteristics and performance parameters of physical functioning and postoperative outcomes for patients undergoing for hepatic resection ( $n=96)$. Abbreviations: $\mathrm{ASA}=$ American Society of Anesthesiologists score; BMI=body mass index; DASI=Duke activity status index; FTSTS=five times sit-to-stand; HGS=handgrip strength; IQR=interquartile range; MET=metabolic equivalent of task; SRT=steep ramp test; TUG=timed up-and-go; VSAQ=veterans-specific activity questionnaire; $W_{\text {peak }}=$ peak work rate; $2 \mathrm{MWT}=$ two-minute walk test. 


\section{Non-surgical complications}

Forty-three postoperative complications were registered in a total of 25 patients (26.0\%). Of these 25 patients, ten (40.0\%) experienced surgical complications, thirteen (52.0\%) had nonsurgical complications and two (8.0\%) experienced both surgical and non-surgical complications. Univariable logistic regression revealed that $\mathrm{BMI} \geq 30 \mathrm{~kg} / \mathrm{m}^{2}$, ASA score III, $\mathrm{CCl}$, aerobic capacity (both absolute and relative SRT performance), muscle strength (FTSTS test), functional mobility (TUG test and 2MWT) and the preoperative perceived level of functional capacity to perform ADL (VSAQ and DASI) were associated with non-surgical complications $(P<0.200)$. The ORs are listed

in Table 4.1. A higher estimated aerobic capacity and a higher level of functional capacity to perform ADL were associated with smaller ORs. Patients with higher scores on the FTSTS test and TUG test were more likely to experience non-surgical complications. If walking distance on the 2MWT increased by $1 \mathrm{~m}$, the OR of experiencing non-surgical complication decreased by 0.028 and with an increase of $25 \mathrm{~m}$ the OR of experiencing non-surgical complications decreased by 0.497 . The area under the curve for the 2 MWT was 0.735 ( $95 \% \mathrm{Cl} 0.607$ to $0.862 ; P=0.004$ ). Further analysis in a multivariable logistic regression model for non-surgical complications was not performed since the insufficient number of events (fifteen events).

\section{Length of hospital stay}

A prolonged length of hospital stay ( $\geq 8$ days) was present in 36 patients (37.5\%). Univariable logistic regression analysis identified that $\mathrm{CCl}$, functional mobility (FTSTS test and 2MWT), and preoperative perceived level of functional capacity to perform ADL (VSAQ and DASI) were associated with a longer length of hospital stay ( $\geq 8$ days) $(P<0.200$, see Table 4.1$)$. The OR for the FTSTS test was 1.139, with an increase of $5 \mathrm{~s}$ on the FTSTS test the OR of a prolonged hospital length of stay is 1.916. With a one-MET difference at the VSAQ, the OR of a prolonged length of hospital stay was 0.874 . Further analysis in a multivariable logistic regression model provided no additional value in predicting a prolonged hospital length of stay. 


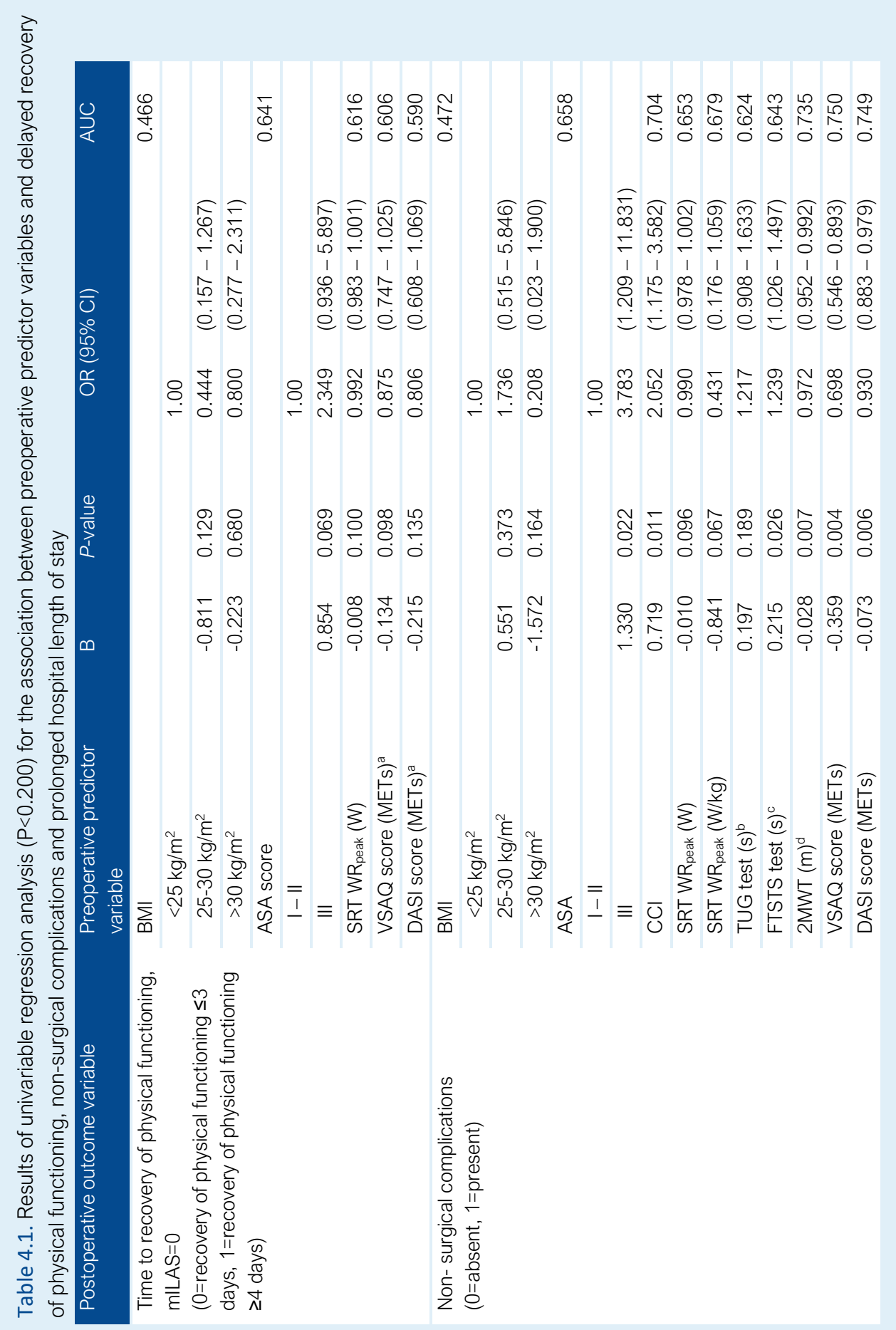




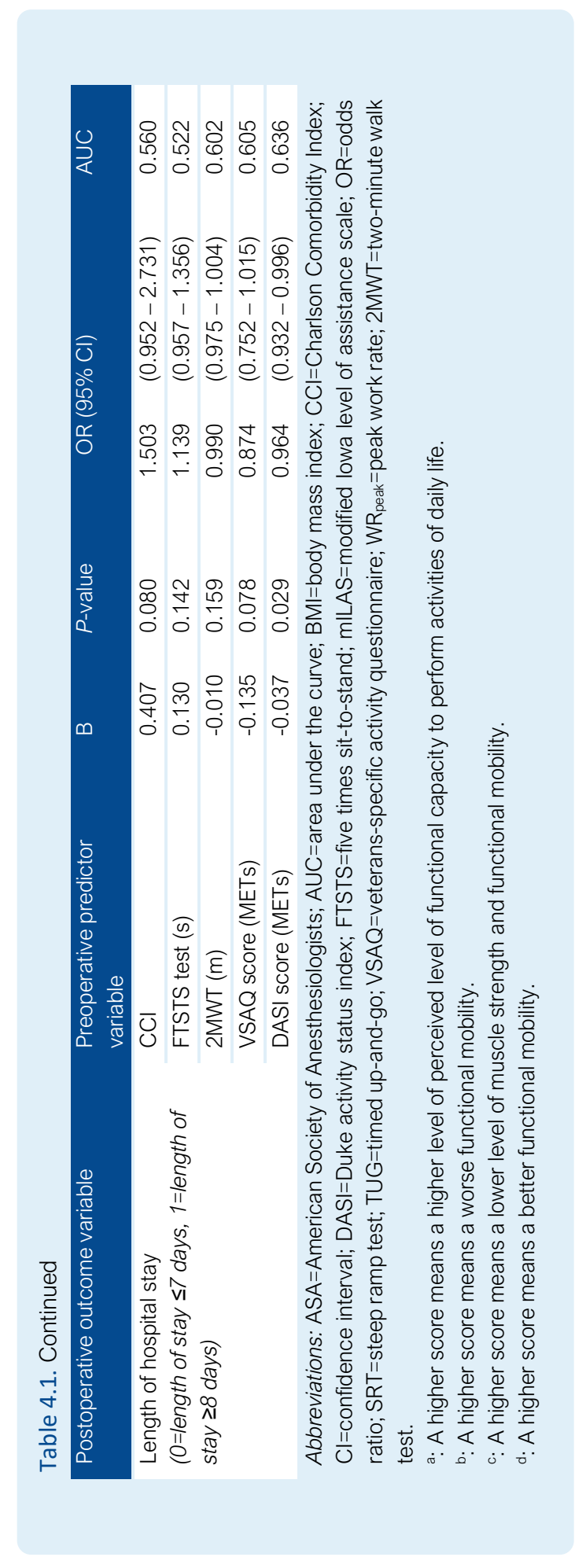




\section{DISCUSSION}

Our study, focusing on preoperative physical functioning and its association with short-term (30day) postoperative outcomes in patients undergoing hepatic resection, revealed potential areas for mitigation of modifiable risk factors. Our data suggest that the performance of a patient on practical performance-based tests of physical functioning, in combination with conventional patient-related characteristics, is associated with postoperative outcomes. A lower estimated aerobic capacity, a lower level of perceived functional capacity to perform ADL, BMI, and ASA score III were independently associated with a delayed time to recovery of physical functioning. Additionally, a lower estimated aerobic capacity, a lower functional mobility, a lower perceived level of functional capacity to perform $\mathrm{ADL}$, a higher $\mathrm{CCl}$, BMI and $\mathrm{ASA}$ score III were independently associated with non-surgical complications. Finally, a lower functional mobility, a lower preoperative perceived level of functional capacity to perform $A D L$, and a higher $C C l$ were associated with a prolonged length of hospital stay. Regarding surgery-related risk factors, patients who underwent laparoscopic surgery in our study showed a shorter time to recovery of physical functioning ( $2.0 \pm 1.0$ days) and length of hospital stay (5.0 \pm 4.0 days) compared to those who had open surgery. With reference to postoperative care management-related risk factors, we found a notable difference between time to recovery of physical functioning and length of hospital stay in both open and laparoscopic surgery (median difference of $3.0 \pm 4.0$ days).

The relation between preoperative performance of patients on the practical performance-based tests of physical functioning and postoperative time to recovery of physical functioning and incidence of postoperative complications is consistent with previously published research in identical patient populations $[5,6,13-15]$. Our data show that a lower aerobic capacity as estimated with the SRT is associated with delayed recovery of physical functioning ( $\geq 4$ days) and non-surgical complications. Measuring a patient's aerobic capacity by CPET (gold standard) is a valuable non-invasive performance test to estimate the risk for postoperative morbidity, mortality, and length of stay in patients undergoing hepatic resection [9-12]. Since preoperative CPET is not yet usual care in our hospital, we use a modified SRT to estimate preoperative aerobic capacity. A previous study in cancer survivors indicated that the prediction of aerobic capacity based on SRT performance is adequate at the group level [25]. Hence, the SRT might be a practical short-time maximal exercise test to identify high- and low risk patients preoperatively. However, further research should investigate whether our modified SRT protocol is a valid test to estimate preoperative aerobic capacity in individual patients undergoing major elective abdominal surgery. Recently, Stuiver et al. [38] concluded that predictions equations do not provide 
sufficiently accurate estimations of aerobic capacity in individual cancer survivors. Moreover, our findings are congruent with other reports confirming the important role of practical performancebased tests in predicting postoperative complications, besides conventional risk factors (ASA score and $\mathrm{CCl}$ ). However, different practical performance-based tests were used [16-18]. For instance, Hayashi et al. [17] showed that a lower 6MWT distance was associated with an increased risk for the event of a major postoperative complication (Clavien-Dindo grade $\geq 3$, with an OR of 1.537) in patients undergoing elective abdominal surgery for hepatic-biliary-disease. Hereto, practical performance-based tests seem useful for preoperative decision-support of patients and their (informal) caregivers, as these are related with postoperative outcomes.

Besides patient-related risk factors, perioperative surgical risk factors should also be taken into account. The ability of the individual patient to respond adequately to the increased metabolic demand due to surgical stress will vary greatly, depending on surgery-related risk factors next to patient-related risk factors. The current study results demonstrate that laparoscopic surgery is accompanied by a shorter time to recovery of physical functioning and a shorter length of hospital stay. However, it should be noted that laparoscopic procedures in our study cohort mainly consisted of less invasive laparoscopic resections (86\% wedge or segment resections). When comparing laparoscopic surgery and open surgery on their short-term outcomes in a recent meta-analysis (ten papers, 2259 patients) shows that laparoscopic surgery is more favorable in terms of morbidity (OR of 0.57 ), length of hospital stay (weighed mean difference (WMD) of -2.13 days), blood loss (WMD of $-124.68 \mathrm{~mL}$ ), and blood transfusion rate (OR of 0.46) [39]. Therefore, surgeons should consider to perform minimally invasive surgery in individuals who are classified as high-risk for adverse postoperative outcomes. Of equal importance as patient- and surgicalrelated risk factors are postoperative care management related risk factors. We found that there is a great discrepancy between the time to recovery of physical functioning and length of hospital stay (in days). The predominant culture of physical inactivity during the clinical recovery phase challenges the psychophysiological system of the patient and contributes to the progressive loss of functional capacity to perform activities of daily life, called hospital-associated disability [4042].

Identifying risk factors in time in the perioperative management for patients undergoing hepatic resection is important to develop targeted perioperative strategies to anticipate and reduce adverse postoperative outcomes. For patient-related risk factors, our results, in addition to those of others, underline the importance of the preoperative level of physical functioning in patients undergoing major abdominal surgery. However, more personalized risk models have to be 
developed. These should incorporate higher volumes of patients, combining medical and functional data, and advanced statistics that can be used in daily clinical practice as clinical decision-support system $[43,44]$. Although such an integral clinical decision support tool is not yet available, we should not refrain high-risk patients from prehabilitation interventions. For patients undergoing hepatic resection, prehabilitation is feasible and results in an improvement of preoperative aerobic capacity [45]. Additionally, knowledge about patients with a low risk of adverse postoperative outcomes should be identified. These low risk patients have to be provided with adequate technology, decision support systems, and minimal support of health care professionals to monitor their physical functioning pre- and postoperatively. To improve surgical and postoperative care management risk factors, attitudes of health care professionals with regard to the inactivating culture and infrastructure should be identified and postoperative care management should focus on facilitating a swift return to adequate performance of ADL [19-20]. Multimodal multidisciplinary ERAS programs describe how perioperative care management can be optimized and underline the importance of early mobilization in the patient's recovery to physical functioning [46].

In conclusion, preoperative physical functioning is associated with short-term (30-day) time to recovery of physical functioning, non-surgical complications, and length of hospital stay in patients undergoing hepatic resection. Consequently, preoperative evaluation of physical functioning seems valuable to establish whether patients have adequate functional reserve capacity to cope with stress during the patient-journey and should play an integral part in the conversation in clinical practice for the decision-making and preparation for surgery. Further exploration is needed on how already established patient related risk-factors can be integrated in a decision-support system which can be used in the preoperative evaluation in daily clinical practice. In the meantime, optimization of peri- and postoperative management in its context and processes is advocated to reduce surgical and postoperative related risk-factors to minimalize the risks of adverse postoperative outcomes. 
1. Parks RW, Garden OJ. Liver resection for cancer. World J Gastroenterol 2001;7:76671.

2. Melloul E, Hubner M, Scott M, Snowden C, Prentis J, Dejong CH, et al. Guidelines for perioperative are for liver surgery: enhanced recovery after surgery (ERAS) Society Recommendations. World J Surg 2016;40:2425-40.

3. Li M, Zhang W, Jiang L, Yang J, Yan L. Fast track for open hepatectomy: a systemic review and meta-analysis. Int J Surg 2016;36:81-9.

4. Dutch Institute for Clinical Auditing (DICA) Leiden. DICA Jaarrapportage 2015: DSCA. accessed January 2017, http://www.clinicalaudit.nl/jaarrapportage/2 015/dsca.html.

5. Dronkers JJ, Chorus AMJ, van Meeteren NLU, Hopman-Rock M. The association of pre-operative physical fitness and physical activity with outcome after scheduled major abdominal surgery. Anaesthesia 2013;68:67-73.

6. Heldens A, Bongers BC, Lenssen AF, Stassen LPS, Buhre WF, van Meeteren NLU. The association between performance parameters of physical fitness and postoperative outcomes in patients undergoing colorectal surgery: an evaluation of care data. Eur J Surg 2017;43:2084-92.

7. Older PO, Levett DZH. Cardiopulmonary exercise testing and surgery. Ann Am Thorac Soc 2017;14:S74-S83.

8. Moran J, Wilson F, Guinan E, McCormick P, Hussey J, Moriarty J. Role of cardiopulmonary exercise testing as a riskassessment method in patients undergoing intra-abdominal surgery: a systematic review. Br J Anaesth 2016; 116:177-91.

9. Snowden CP, Prentis J, Jacques B, Anderson $\mathrm{H}$, Manas $\mathrm{D}$, Jones $\mathrm{D}$, et al. Cardiorespiratory fitness predicts mortality and hospital length of stay after major elective surgery in older people. Ann Surg 2013;257:999-1004.
10. Junejo MA, Mason JM, Sheen AJ, Moore J, Foster P, Atkinson D, et al.

Cardiopulmonary exercise testing for preoperative risk assessment before hepatic resection. Br J Surg 2012;99:1097-104.

11. Kasivisvanathan $\mathrm{R}$, Abbassi-Ghadi $\mathrm{N}$, McLeod AD, Oliver A, Rao Baikady R, Jhanji S, et al. Cardiopulmonary exercise testing for predicting postoperative morbidity in patients undergoing hepatic resection surgery. HPB (oxford) 2015;17:637-43.

12. Ulyett S, Wiggans MG, Bowles MJ, Aroori $S$, Briggs $C D$, Erasmus $P$, et al. Clinical assessment before hepatectomy identifies high-risk patients. J Surg Res 2015;198:87-92.

13. Elings J, van der Sluis G, Goldbohm S, Galindo-Garre F, Gast A, Hoogeboom T, et al. Development of a risk stratification model for delayed inpatient recovery of physical activities in patients undergoing total hip replacement. J Orthop Sports Phys Ther 2016;46:135-43.

14. Oosting E, Hoogeboom T, Appelman S, Swets A, Dronkers J, Meeteren N. Preoperative prediction of inpatient recovery of function after total hip arthroplasty using performance-based tests: a prospective cohort study. Disabil Rehabil 2015; 38:1243-9.

15. van der Sluis G, Goldbohm R, Elings J, Nijhuis-van der Sanden M, Akkermans R, Bimmel R, et al. Pre-operative functional mobility as an independent determinant of inpatient functional recovery after total knee arthroplasty during three periods that coincided with changes in clinical pathways. Bone Joint J 2017;99-B: 21117.

16. Huisman MG, van Leeuwen BL, Ugolini G, Montroni I, Spiliotis J, Stabilini C, et al. "Timed Up \& Go": a screening tool for predicting 30-day morbidity in oncogeriatric surgical patients? A multicenter cohort study. PLoS One 2014;9:e86863. 
17. Hayashi K, Yokoyama $\mathrm{Y}$, Nakajima $\mathrm{H}$, Nagino M, Inoue T, Nagaya $M$, et al. Preoperative 6-minute walk distance accurately predicts postoperative complications after operations for hepatopancreato-biliary cancer. Surgery 2017;161:525-32.

18. Reddy S, Contreras CM, Singletary B, Bradford TM, Waldrop MG, Mims AH, et al. Timed Stair Climbing Is the Single Strongest Predictor of Perioperative Complications in Patients Undergoing Abdominal Surgery. J AM Coll Surg. 2016;222:559-66.

19. Hulzebos EH, van Meeteren NL. Making the elderly fit for surgery. Br J Surg 2016;103:463.

20. Hulzebos EH, van Meeteren NL. Making the elderly fit for surgery. Br J Surg 2016; 103:e12-5.

21. Carli F, Ferreira V. Prehabilitation: a new area of integration between geriatricians, anesthesiologists, and exercise therapists. Aging Clin Exp Res 2018;30:241-4.

22. Shields RK, Enloe LJ, Evans RE, Smith KB, Steckel SD. Reliability, validity, and responsiveness of functional tests in patients with total joint replacement. Phys ther 1995;75:169-76.

23. Thomas S, Reading J, Shephard RJ. Revision of the Physical Activity Readiness Questionnaire (PAR-Q). Can J Sport Sci 1992;17:338-45.

24. Bongers BC, Takken T. The paediatric version of the steep ramp test. J Physiother 2014;60:113.

25. De Backer IC, Schep G, Hoogeveen A, Vreugdenhil G, Kester AD, van Breda E. Exercise testing and training in a cancer rehabilitation program: the advantage of the steep ramp test. Arch Phys Med Rehabil 2007;88:610-16.

26. Meyer K, Samek L, Schwaibold M, Westbrook S, Hajric R, Lehmann M, et al. Physical responses to different modes of interval exercise in patients with chronic heart failure--application to exercise training. Eur Heart J 1996;17:1040-7.
27. Bohannon RW. Reference values for the five-repetition sit-to-stand test: a descriptive meta-analysis of data from elders. Percept Mot Skills 2006;103:21522.

28. Lord SR, Murray SM, Chapman K, Munro B, Tiedemann A. Sit-to-Stand Performance Depends on Sensation, Speed, Balance, and Psychological Status in Addition to Strength in Older People. J Gerontol A Biol Sci Med Sci 2002;57:M539-43.

29. Tiedemann A, Shimada H, Sherrington C, Murray S, Lord S. The comparative ability of eight functional mobility tests for predicting falls in community-dwelling older people. Age Ageing 2008;37:430-5.

30. Peolsson A, Hedlund R, Oberg B. Intraand inter-tester reliability and reference values for hand strength. J Rehabil Med 2001;33:36-41.

31. Brooks D, Davis AM, Naglie G. Validity of 3 physical performance measures in inpatient geriatric rehabilitation. Arch Phys Med Rehabil 2006;87:105-10.

32. Bohannon RW, Wang YC, Gershon RC Two-minute walk test performance by adults 18 to 85 years: normative values, reliability, and responsiveness. Arch Phys Med Rehabil 2015;96:472-7.

33. Podsiadlo D, Richardson S. The Timed "Up \& Go": A Test of Basic Functional Mobility for Frail Elderly Persons. J Am Geriatr Soc 1991;39:142-8.

34. de Carvalho Bastone A, de Souza Moreira B, Teixeira CP, Dias JM, Dias RC. Is the Veterans Specific Activity Questionnaire Valid to Assess Older Adults Aerobic Fitness? J Geriatr Phys Ther 2016; 39:11724.

35. Hlatky MA, Boineau RE, Higginbotham MB, Lee KL, Mark DB, Califf RM, et al. A brief self-administered questionnaire to determine functional capacity (the Duke Activity Status Index). Am J Cardiol 1989;64:651-4.

36. Steyerberg EW. Statistical models for prediction. In: Steyerberg EW, editor. Clinical Prediction Models: A Practical Approach to Development, Validation, and Updating. 1st ed. New York: SpringerVerlag; 2009, p.53-83. 
37. Steyerberg EW, Eijkemans MJ, Harrell FE Jr, Habbema JD. Prognostic modelling with logistic regression analysis: a comparison of selection and estimation methods in small data sets. Stat Med. 2000;19:105979.

38. Stuiver MM, Kampshoff CS, Persoon S, Groen W, van Mechelen W, Chinapaw MJM, et al. Validation and refinement of prediction models to estimate exercise capacity in cancer survivors using the steep ramp test. Arch Phys Med Rehabil 2017;98:2167-73.

39. Zhang X-L, Liu R-F, Zhang D, Zhang Y-S, Wang $T$. Laparoscopic versus open liver resection for colorectal liver metastases: a systematic review and meta-analysis of studies with propensity score-based analysis. Int J Surg 2017;44:191-203.

40. Dronkers J, Witteman B, van Meeteren N. Surgery and functional mobility: doing the right thing at the right time. Tech Coloproctol 2016;20:339-41.

41. Covinsky KE, Pierluissi E, Johnston C. Hospitalization-associated disability: "she was probably able to ambulate, but i'm not sure". Jama 2011; 306:1782-93.
42. Sourdet $\mathrm{S}$, Lafont $\mathrm{C}$, Rolland $\mathrm{Y}$, Nourhashemi F, Andrieu S, Vellas B.

Preventable iatrogenic disability in elderly patients during hospitalization. J Am Med Di Assoc 2015;16:674-81.

43. Lambin $P$, Zindler J, Vanneste BGL, De Voorde LV, Eekers D, Compter I, et al. Decision support systems for personalized and participative radiation oncology. Adv Drug Deliv Rev 2017;109:131-53.

44. Jones BD, Jones R, Dunne DF, Astles T, Fenwick SW, Poston GJ et al. Patient selection a perioperative optimisation in surgery for colorectal liver metastases. Eur Surg 2018;50:87-92.

45. Dunne DF, Jack S, Jones RP, Jones L, Lythgoe DT, Malik HZ, et al. Randomized clinical trial of prehabilitation before planned liver resection. Br J Surg 2016;103:504-12.

46. Ljungqvist $\mathrm{O}$, Scott $\mathrm{M}$, Fearon $\mathrm{KC}$. Enhanced recovery after surgery: A review. JAMA Surg 2017;152:292-8. 
Een postoperatieve complicatie is een 'event' dat 'impact' heeft op het fysiek functioneren van de patiënt, waardoor het essentieel is om ook deze 'impact' per patiënt per complicatie te gaan registreren, incluis de interventies die zich op het 'event' en zijn 'impact' richten. 


\section{Chapter 5}

Exploring the relation between preoperative physical functioning and the impact of major complications in patients following pancreatic resection

Christel A. van Beijsterveld | Bart C. Bongers | Marcel den Dulk | Sander M. van Kuijk | Cornelius H. Dejong | Nico L. van Meeteren 


\section{ABSTRACT}

\section{Background}

This study aimed to evaluate the association between preoperative level of physical functioning and time to recovery of physical functioning, postoperative complications, and the impact of postoperative major complications in patients undergoing elective pancreatic resection. Additionally, prediction models to identify high-risk patients for developing a major complication were externally validated.

\section{Methods}

Perioperative data of patients who underwent pancreatic resection were analysed. Primary outcomes were time to recovery of physical functioning and postoperative major complications. Impact of a major complication was explored by evaluating its effect on time to recovery of physical functioning. Risk-prediction models were retrieved following a systematic review.

\section{Results}

Multivariable analysis ( $n=63)$ showed that ASA grade III (OR 3.498) and preoperative platelet count (OR 1.005) were associated with major complications, whereas aerobic capacity (OR 0.347) was associated with time to recovery of physical functioning. Age, preoperative aerobic capacity, functional mobility, and perceived level of functional capacity were associated with the impact of a major complication. The AUC of two risk prediction models were 0.556 and 0.701 .

\section{Conclusion}

Preoperative parameters of physical function were associated with postoperative outcomes and may be useful in outcome prediction, although future approaches should not only register the incidence of major complications but also take the impact of a complication on a patient's physical functioning into account. 


\section{INTRODUCTION}

Patients diagnosed with cancer in the pancreas often experience disease-related symptoms (e.g., loss of body mass, jaundice, loss of appetite, nausea), which also induce changes in skeletal muscle mass and in the function of a variety of organs leading to functional impairments [1]. Surgical resection is the only potentially curative modality. However, pancreatic resection is associated with a high risk of perioperative morbidity (36-60\%) and mortality (2.9-3.5\%) [2-4]. Patients and their (in)formal caregivers should together make a well-informed decision about the risk-benefit ratio for undergoing major abdominal surgery with or without preoperative preventive interventions, and the options for additional or non-surgical cancer-directed treatment as a (palliative) alternative $[5,6]$. Several prediction models exist to preoperatively estimate the risk of postoperative morbidity and mortality [7-9], which can be used to support decision-making with the patient and their (in)formal caregivers when considering pancreatic resection. However, there is a wide variability in the selection of preoperative factors and the definition of postoperative outcomes in risk models. Some have included operative factors (e.g., type of surgery, operation time, blood loss) $[10,11]$, making them more difficult to utilize during preoperative counselling of patients. Other risk models focus merely on one specific type of complication, mostly pancreatic fistula $[12,13]$. Moreover, as external - pragmatic clinical - validation of available evidence-based risk models is scarce and risk models are thought to not outweigh the surgeon's assessment, they currently play a very limited role in clinical practice $[14,15]$.

Patient's tolerance for surgery depends on their ability to adapt to the stress of hospitalization (e.g. decreased physical activity [16]) and surgery (e.g. hormonal and metabolic disturbances [17]). Especially those patients developing a complication after pancreatic surgery should have enough reserve capacity to cope with the increased psychophysiological load [18, 19]. Some studies have shown that preoperative aerobic capacity is associated with postoperative morbidity, mortality, and length of stay in patients undergoing pancreatic resection [20-22]. Besides aerobic capacity, also muscle strength, functional mobility, and the patient's physical activity level in daily life are considered as indicators for the adaptive capacity of the patient, as they are associated with postoperative time to recovery of physical functioning, morbidity, and mortality following major abdominal surgery [23-25]. If a patient has significant perioperative risk factors (e.g., low aerobic fitness, malnutrition, anemia), appropriate preoperative preventive interventions should be initiated by means of personalized multimodal prehabilitation. Optimizing a patient's psychophysiological reserves prior to surgery is increasingly considered beneficial for recovery functioning, as it reduces the risk for morbidity, and mortality following major abdominal 
surgery [26, 27]. Furthermore, it is hypothesized that psychophysiological reserve capacity is associated with the impact of any complication, in which the impact of a postoperative event is expected to be lower in patients with higher levels of physical fitness. The first aim of this study was to evaluate the association between the preoperative level of physical functioning and postoperative outcomes in patients with pancreatic cancer opting for pancreatic resection. In addition, we aimed to assess the external validity of prediction models that have been developed to preoperatively identify patients with a higher risk for developing a major complication after pancreatic resection. The number of patients who develop complications after pancreatic resection is high and fitter patients might also develop a complication. It should be noted that fitter patients might demonstrate enhanced coping with a complication, reducing its impact and resulting in better postoperative outcomes. Hence, the final aim of the current study was to explore whether the patient's preoperative level of physical functioning was associated with the impact of major postoperative complications.

\section{METHODS}

\section{Study design}

This single-center prospective cohort study evaluated postoperative outcomes (time to recovery of physical functioning, major complications and their impact) in patients with pancreatic or periampullary carcinoma undergoing elective pancreatic resection.

\section{Participants}

Patients with pancreatic tumors (adenocarcinoma of the pancreatic head, duodenum, ampulla of Vater, and lower bile duct, or neuro-endocrine tumors), primary benign tumors (e.g., intraductal papillary mucinous neoplasm of the pancreas), and metastases in the pancreas who were scheduled for curative pancreatic resection based on input from a multidisciplinary team of medical specialist (surgeons, oncologists, radiologists, pathologist, radiotherapist, gastroenterologists) at the Maastricht University Medical Center (Maastricht UMC+) between March 2016 and July 2018 were included. Exclusion criteria were pancreatic resection for acute or chronic pancreatitis (except when there was a suspicion for malignancy), a primary tumor outside the pancreas with only secondary involvement of the pancreas, pancreas preserving bile duct resection, and exploratory surgery without pancreas resection." Additionally, patients who had not undergone screening of physical functioning as part of preoperative assessment because of logistical issues, or those who were screened on their level of physical functioning, but did not opt for surgery, were excluded. All consecutive patients with no objection for using their usual 
care data for research purposes were included. The medical ethical committee of the Maastricht UMC+ decided (reference number 15-4-234) that this study met the ethical policies of the Maastricht UMC+.

\section{Patient journey}

Patients scheduled for curative pancreatic resection were consecutively referred to the outpatient physical therapy department by the hepato-pancreato-biliary (HPB) surgeon or clinical nurse specialist for screening of physical functioning as part of multidisciplinary preoperative assessment. A hospital physical therapist with expertise in the hepato-pancreato-biliary (HPB) surgical field conducted the preoperative screening. The preoperative screening included tests to estimate the patient's aerobic capacity (modified steep ramp test [SRT], a short-time maximal exercise test on a cycle ergometer), muscle strength (handgrip strength [HGS]), functional mobility (five times sit-to-stand [FTSTS] test, timed up-and-go [TUG] test, and 2-minute walking test [2MWT]), and perceived level of functional capacity to perform activities of daily life (veteransspecific activity questionnaire [VSAQ] and Duke activity status index [DASI], both expressed in metabolic equivalents of task [METs]). An illustration of the patient journey and an extensive description of the used measurement protocols for the preoperative screening of physical functioning is provided in Appendix 5.1.

All patients were educated (verbally and by using information leaflets) regarding the importance of an adequate preoperative level of physical functioning, and were advised to stay physically active throughout the pre- and postoperative phase. All patients were routinely admitted one day prior to surgery to the HPB surgical ward. Postoperatively, patients were admitted to the HPB surgical ward as soon as possible, unless admission to the intensive care unit was warranted due to intra-operative events or postoperative complications. All patients received postoperative physical therapy once a day (starting at postoperative day 1) focusing on airway clearing exercises and regaining functional independence by early mobilisation, adapted to the individual patient's needs and progress [28-30]. Recovery of physical functioning was monitored using the modified lowa level of assistance scale (mILAS), scoring on the ability to perform five functional tasks [31, 32]. Patients were discharged when analgesia, wound healing, organ functions, laboratory parameters, dietary intake, and recovery of physical functioning (mILAS) were considered adequate by the clinical team. 
Pre- and postoperative outcomes, including monitoring of the patient's level of physical functioning

Preoperative clinical data collected from all patients consisted of patient demographics and anthropometrics (e.g., age, sex, body height, body mass), comorbidities, American Society of Anesthesiologists (ASA) grade, functional status (scored on the eastern cooperative oncology group [ECOG] scale [33]), smoking status, chronic steroid use, preoperative sepsis, preoperative interventions (e.g., neoadjuvant treatment, biliary drainage, nutritional support), preoperative blood parameters (e.g., hemoglobin concentration, white blood cell count, platelet count, creatinine level, albumin level), and preoperative level of physical functioning (e.g., aerobic capacity, handgrip strength, functional mobility, and perceived level of functional capacity to perform activities of daily life). These preoperative variables were selected based on a literature search [34-36] and usual care procedures as applied by the department of HPB surgery and department of physical therapy.

Postoperative data included pathology-specific information, surgical procedures, time to recovery of physical functioning, postoperative complications, and length of hospital stay. Time to recovery of physical functioning was defined as the time between the day of surgery and the day at which patients reached a mILAS score of 0 (in days). Time to recovery of physical functioning was also dichotomized according to the median time to recovery of physical functioning. Postoperative complications were classified following the criteria of the international study group for pancreatic surgery (ISGPS) for pancreatic fistula [37], delayed gastric emptying [38], hemorrhage [39], and chyle leakage [40]. Biliary leakage was classified according to the definition of the international study group of liver surgery [41]. The incidence of postoperative complications as events were graded using the Clavien-Dindo classification [42]. Complications graded with I or II were defined as minor and complications between III and $\mathrm{V}$ were defined as major. Impact of these events, especially the major complications, was explored by calculating composite endpoints.

\section{Selection of risk models to predict risk for major complications}

The transparent reporting of a multivariable prediction model for individual prognosis or diagnosis (TRIPOD) guideline was used for the external validation of available risk models [43]. A systematic literature search was performed in PubMed to identify cohort studies developing models to predict the risk for major complications following pancreatic resection published within the last ten years (Appendix 5.2). Studies were included when they 1) comprised a study population of patients (>18 years) undergoing elective pancreatic resection, 2 ) evaluated preoperative patient-related 
risk factors in relation to postoperative morbidity, 3) provided quantitative indicators concerning the association between preoperative patient-related variables and postoperative morbidity, and 4) proposed a clinically interpretable risk-model. Studies were excluded when they merely predicted one particular complication (e.g., pancreatic fistula) or used intraoperative predictors. The study selection process is available in Appendix 5.2, Table 5A.2.

Association between preoperative physical functioning and the impact of postoperative major complications

The impact of a major complication was explored by evaluating its effect on time to recovery of physical functioning. Hereto, two composite endpoints were defined to enable the comparison of two subgroups of patients with a different impact of a major complication. One group of patients with a major complication and a time to recovery of physical functioning above its median value in this study cohort (higher impact) and a group of patients with a major complication and a time to recovery of physical functioning below its median value (lower impact). To explore whether a patient's preoperative level of physical functioning was associated with the impact of a major postoperative complication, preoperative characteristics were compared between the two subgroups.

\section{Statistical analysis}

The Statistical Package for the Social Sciences for Windows (version 23.0; IBM, SPSS Inc., Chicago, IL, USA) was used for statistical analysis. For continuous variables, normality was tested with the Kolmogorov-Smirnov test. Missing preoperative laboratory values or results on preoperative physical functioning tests were imputed using multiple imputation with ten iterations for each imputation. To analyse the association between preoperative level of physical functioning and postoperative outcomes, the Mann-Whitney $U$ test was used for continuous variables and Fisher's exact test was used for categorical variables. All variables with P-values $<0.05$ in univariate analysis were included in a multivariable logistic regression model with backward stepwise elimination (with ten events per variable). A combination of conventional and physical functioning predictors were chosen based on level of statistical significance and expert opinion. To evaluate the performance of the available risk models, discrimination and calibration of the risk scores were assessed for each model [44]. Receiver operating characteristics (ROC) curve analysis was performed, and the discriminative ability of the prediction models was quantified as the area under the curve (AUC). The model's calibration evaluates the agreement between the observed probability and predicted probability of postoperative complications, and is illustrated 


\section{Chapter 5}

using a calibration plot. A regression coefficient of 1 and an intercept of zero represents perfect calibration of the model. To explore the impact of a major complication, the two subgroups of patients with a different impact of a major complication were compared using the Mann-Whitney $U$ test for the continuous variables and the Fisher's exact test for categorical variables. A $P$-value $<0.05$ was considered statistically significant.

\section{RESULTS}

Pre- and postoperative outcomes with monitoring of the patient's level of physical functioning A total of 79 patients diagnosed with pancreatic or periampullary carcinoma and opting for resection with preoperative assessment of physical functioning between February 2016 and July 2018 were considered for the study. Sixteen patients (20.3\%) were excluded retrospectively, as intraoperative findings resulted in no surgical resection being performed. Hence, 63 patients $(79.7 \%)$ were available for analysis following pancreatic resection. Mean \pm SD time between preoperative screening of physical functioning and surgery was $23.6 \pm 21.0$ days. Patient demographics, preoperative variables, and postoperative outcomes of the study cohort are listed in Table 5.1. Figure 5.1 gives an illustrative overview of the pre-and postoperative level of physical functioning of three individual patients following pancreatic resection for pancreatic cancer. $A$ total of 44 patients (69.8\%) developed a complication of which major complications (ClavienDindo classification $\geq$ III) occurred in 27 patients (42.9\%). The median time to recovery of physical functioning was 6.5 days (range 1-49) and median length of hospital stay was 14 days (range 678 days). 


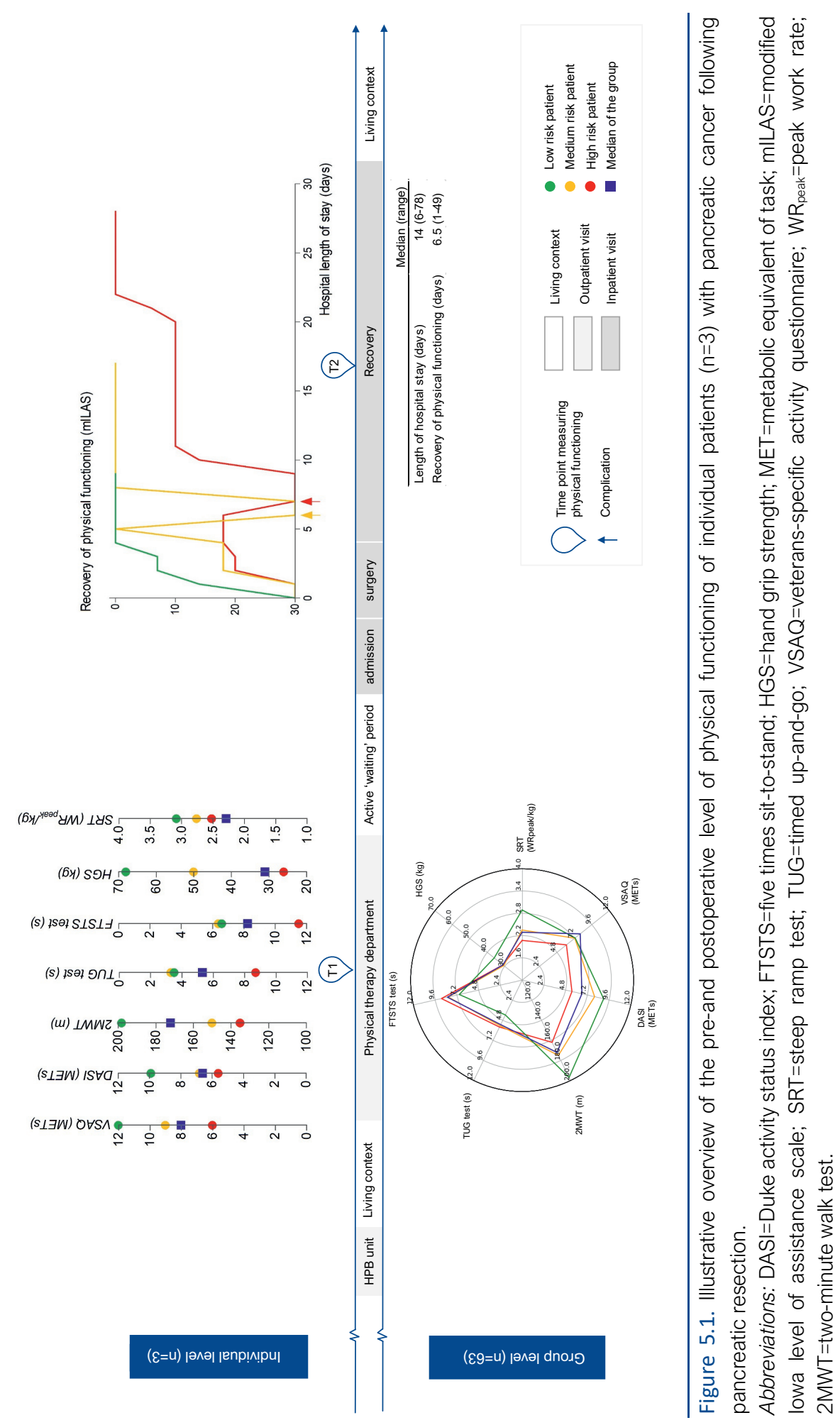




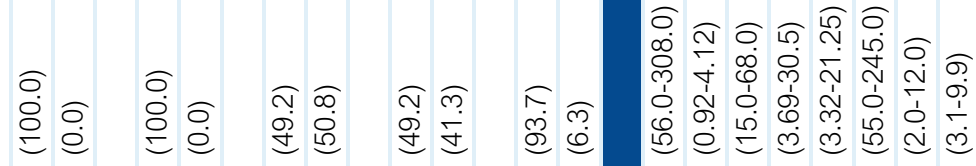

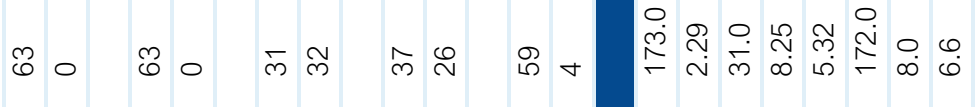

远

능

굴

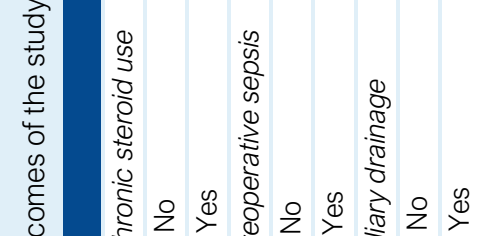

:

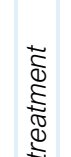

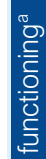

世㐫

응

党 $\frac{1}{0}$

in

效 d

.

일

क

ले

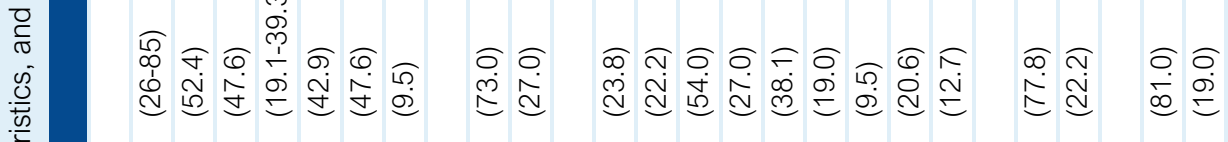

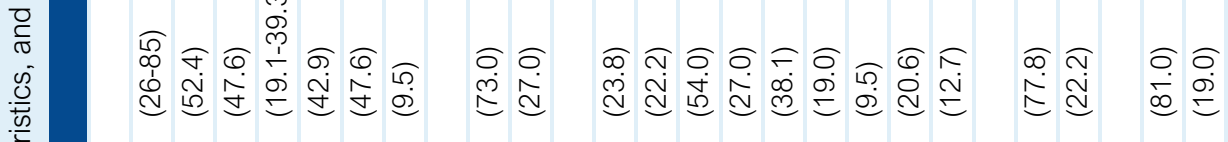

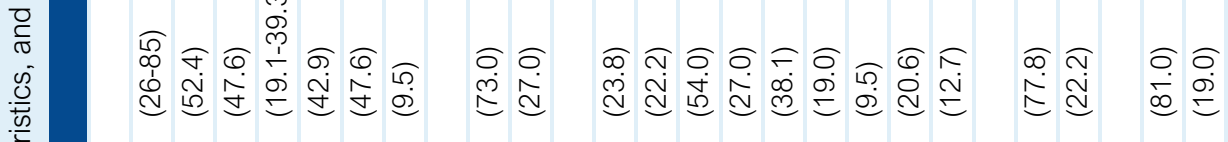

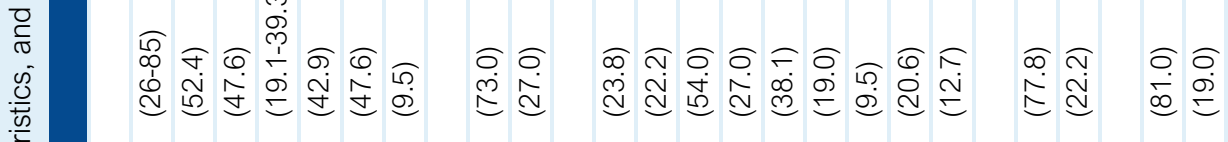

व) के

(n)

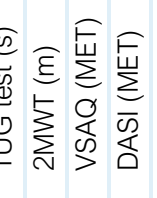

Q

号?

至

爻

ग) व

용

$\sum_{0} \frac{i}{\frac{\pi}{\sigma}}$

政

ริ

ले

ป

ఖ

잉

क्

은

ठำ

๑

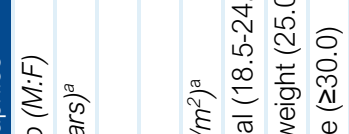

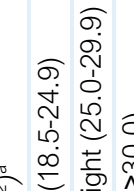

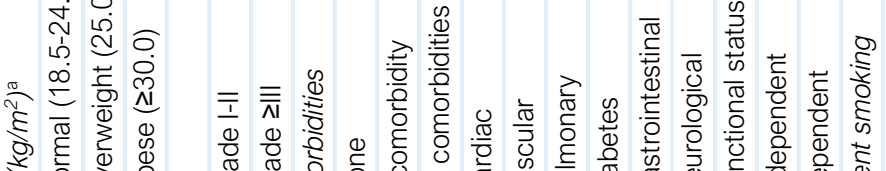

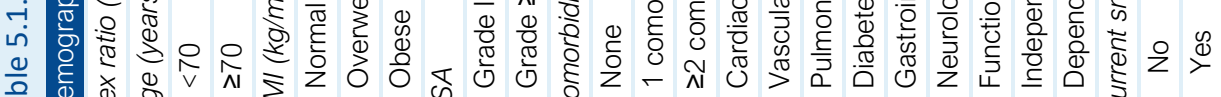

这 ते $\frac{\pi}{0}$ ठ 달 产 离 자 ब के के वैं 는 3 ๗ँ के 응 후 . व 11 음 한당 


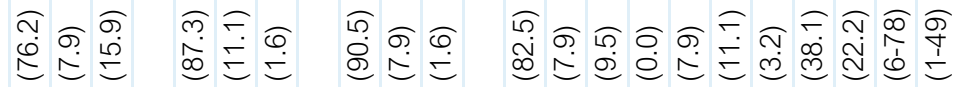

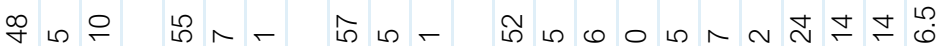

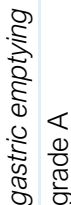

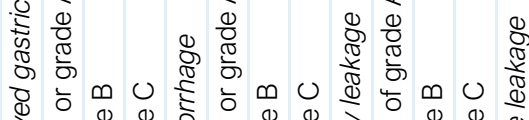

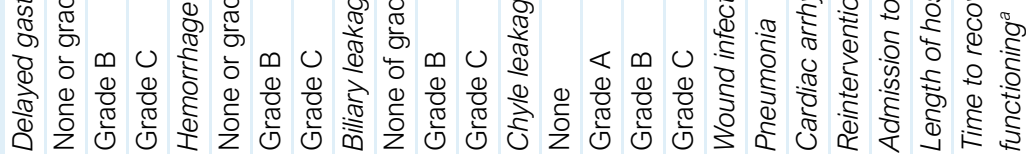

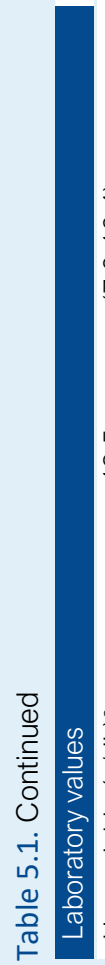

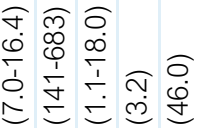

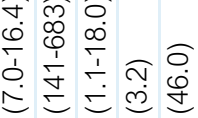

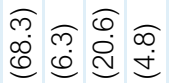

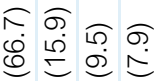

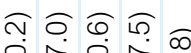

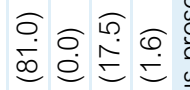

$$
\stackrel{m}{\forall}+\stackrel{m}{\longrightarrow} m
$$

$\stackrel{Y}{\Upsilon} 0\llcorner$

$\stackrel{m}{\leftarrow} \mp m$

is $0=-$

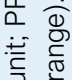

选 $\frac{\sqrt{0}}{\frac{0}{0}}$

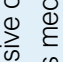

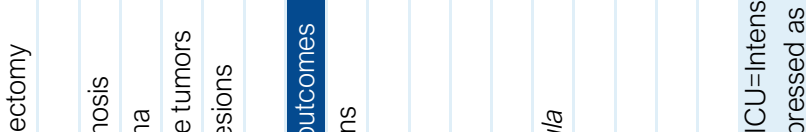

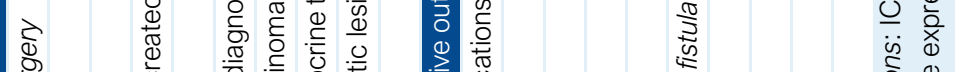

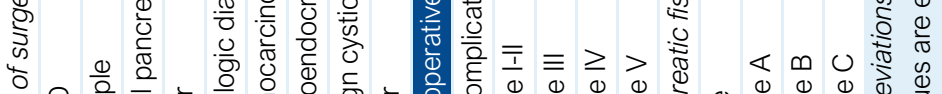

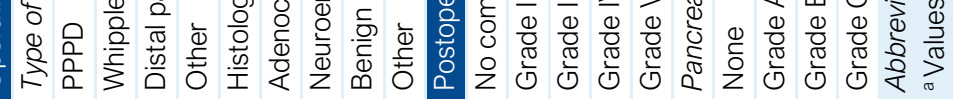


Univariable analysis (see Table 5.2) demonstrated that age, ASA grade (III versus I-II), and preoperative platelet count $\left(10^{3}\right.$ cells $\left./ \mathrm{mm}^{3}\right)$ were associated with the occurrence of major complications $(P<0.05)$. Multivariable logistic regression with stepwise elimination yielded a model including two variables predicting these events. ASA grade III versus I-II (odds ratio [OR] of 1.498; 95\% confidence interval [Cl] of 1.033 to $11.852 ; P=0.044)$ and preoperative platelet count (OR of 1.005 per $10^{3}$ cells $/ \mathrm{mm}^{3} ; 95 \% \mathrm{Cl}$ of 1.000 to $1.010 ; P=0.038$ ) (see Table 5.3). The AUC for this model was $0.740(95 \% \mathrm{Cl} 0.617$ to $0.863 ; P=0.001)$. Furthermore, univariable analysis for recovery of physical functioning showed that age, aerobic capacity (relative SRT performance, $\mathrm{WR}_{\text {peak }}$ in $\mathrm{W} / \mathrm{kg}$ ), functional mobility (2MWT distance, in $\mathrm{m}$ ), preoperative perceived level of functional capacity to perform ADL (VSAQ and DASI, both in METs), and preoperative white blood cell count $\left(10^{3}\right.$ cells $\left./ \mathrm{mm}^{3}\right)$ were associated with mILAS-data for time to recovery of physical functioning (see Table 5.2). Multivariable logistic regression with stepwise elimination starting with three variables (age in years, SRT performance in $\mathrm{W} / \mathrm{kg}$, and 2MWT distance in $\mathrm{m}$ ) yielded a model predicting time to recovery of physical functioning above its median value in this study cohort from relative SRT performance (OR of $0.347 ; 95 \% \mathrm{Cl} 0.141-0.854 ; P=0.021$ ). The AUC for relative SRT performance was 0.680 ( $95 \% \mathrm{Cl} 0.545$ to $0.815 ; P=0.014$ ). 


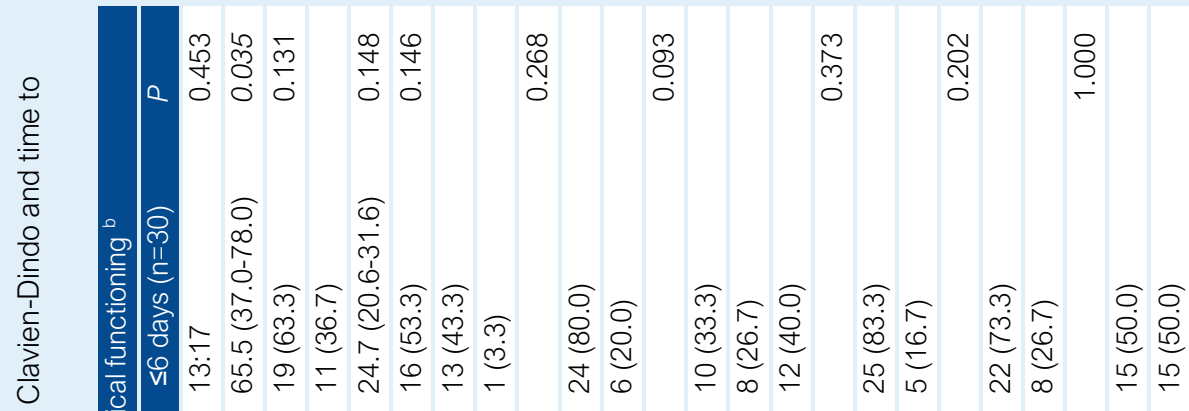

○

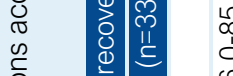

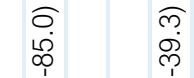

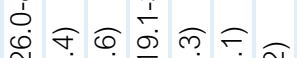

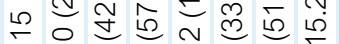

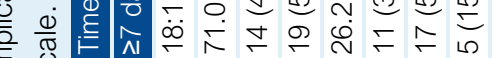

กิ

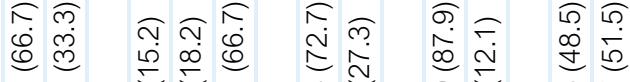

कำ

ล2

ก

ฟ

iे

$\underline{\sim}$

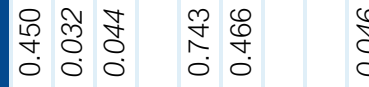

$\begin{array}{lll}0 & \infty \\ 0 & 0 & 0 \\ 0 & 0 & 0 \\ 0 & 0 & 0\end{array}$

$\stackrel{\stackrel{0}{\sim}}{\frac{1}{0}}$

$\bar{n}$
$\substack{0 \\ 0}$

$\bar{\delta}$
$\infty$
0

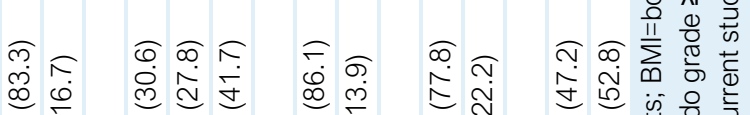

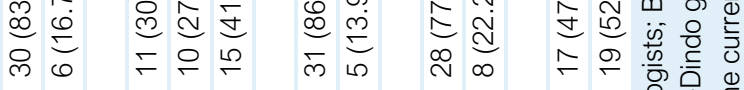

菅

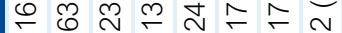

m

$\sim \infty$

응 눙

站 $\frac{\pi}{0}$

อิ क

एक

@ั $\simeq 0 \hat{0}$ e 
$\begin{array}{lll}\substack{1 \\ 0 \\ 0} & 0 \\ 0 & 0 \\ 0\end{array}$

হ.

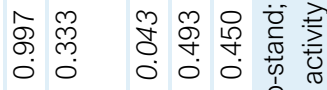

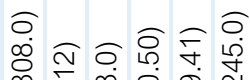

m

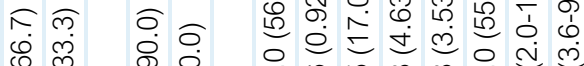
๑

(ब) ल

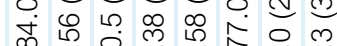

은 츰

ọ:

mे

का 0 बैं

占这 के

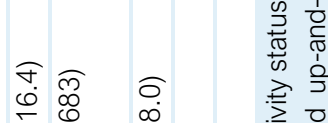

$\vdash \stackrel{ }{\sim} \stackrel{m}{-}$

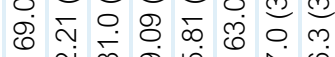

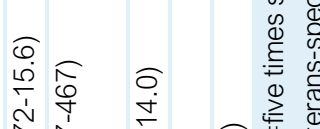

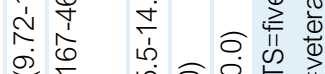

o 0

심 $\stackrel{\infty}{\sim}$

×

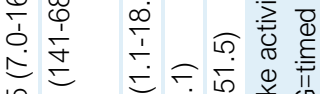

L

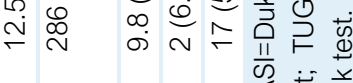

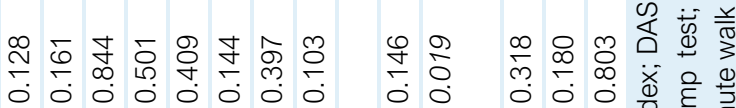

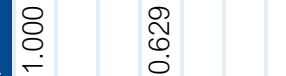

$\circ 0^{\circ}$

离 है

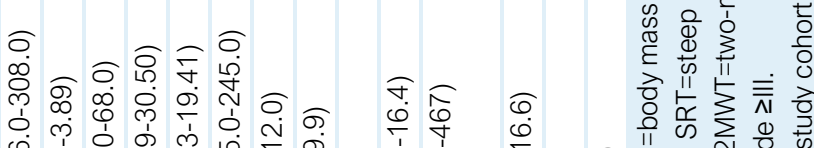

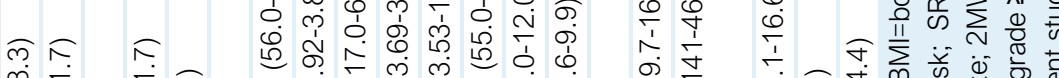

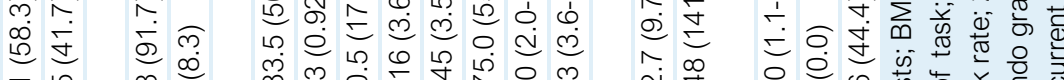

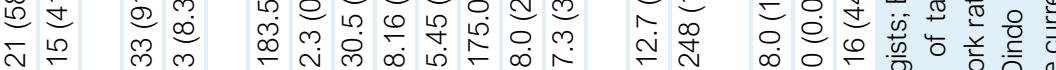

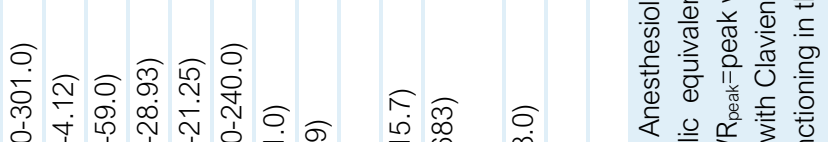

लำ 성 d d

मํㅇㅁㅇ बै $\delta$ 迎

เి 0

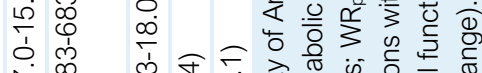

대

- 0 年

$\pm \stackrel{\infty}{=}$

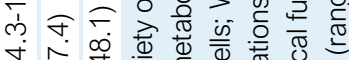

m.

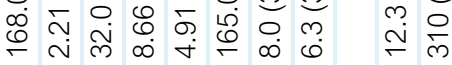

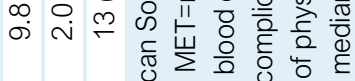

$0 \quad$ है

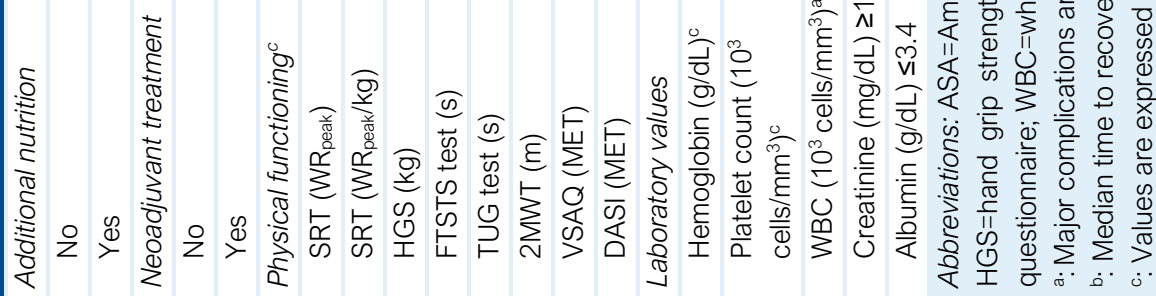




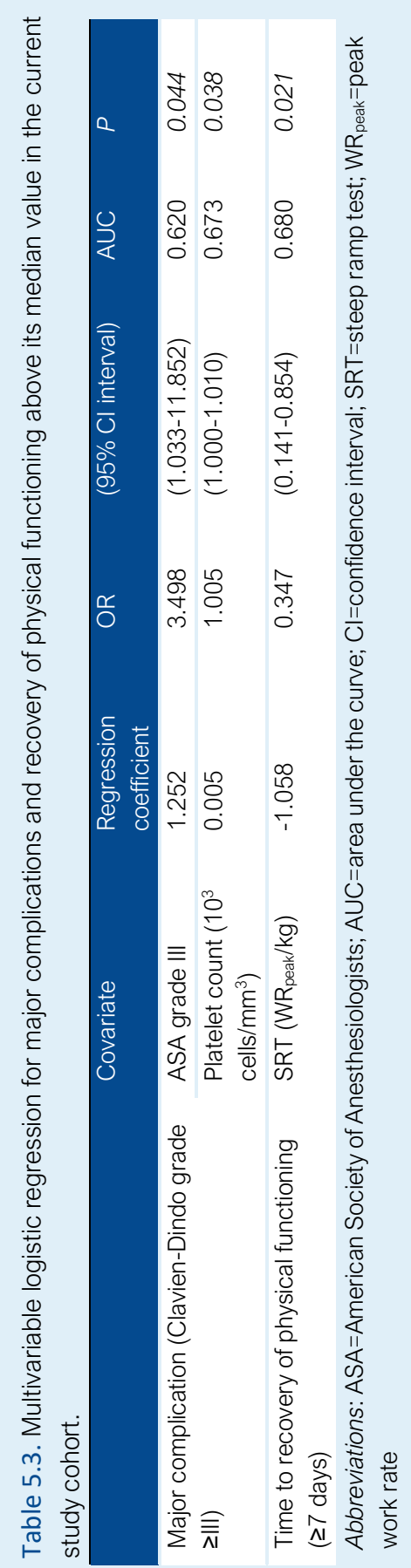




\section{Selection and performance of risk prediction models}

Three preoperative risk prediction models for pancreatic surgery were identified in the literature (Appendix 5.2, Figure 5A.2) [8, 9, 34]. The number of predictors ranged from five to ten (Appendix 5.2, Table 5A.3 and Figure 5A.4). One model could not be validated because of insufficient information, even after contacting the authors [8]. The authors closed the online risk prediction tool, as it was based on "outdated information". Hence, two models [9, 32] were externally validated within the present study cohort. The models had a discriminative ability (AUC) of 0.701 (0.565-0.838) [9] and 0.556 (0.413-0.698) [32], respectively. The calibration plots showed a poor agreement between the observed and predicted probabilities, and both models on average - underestimated the risk of major complications in the present study cohort (see Figure 5.2).
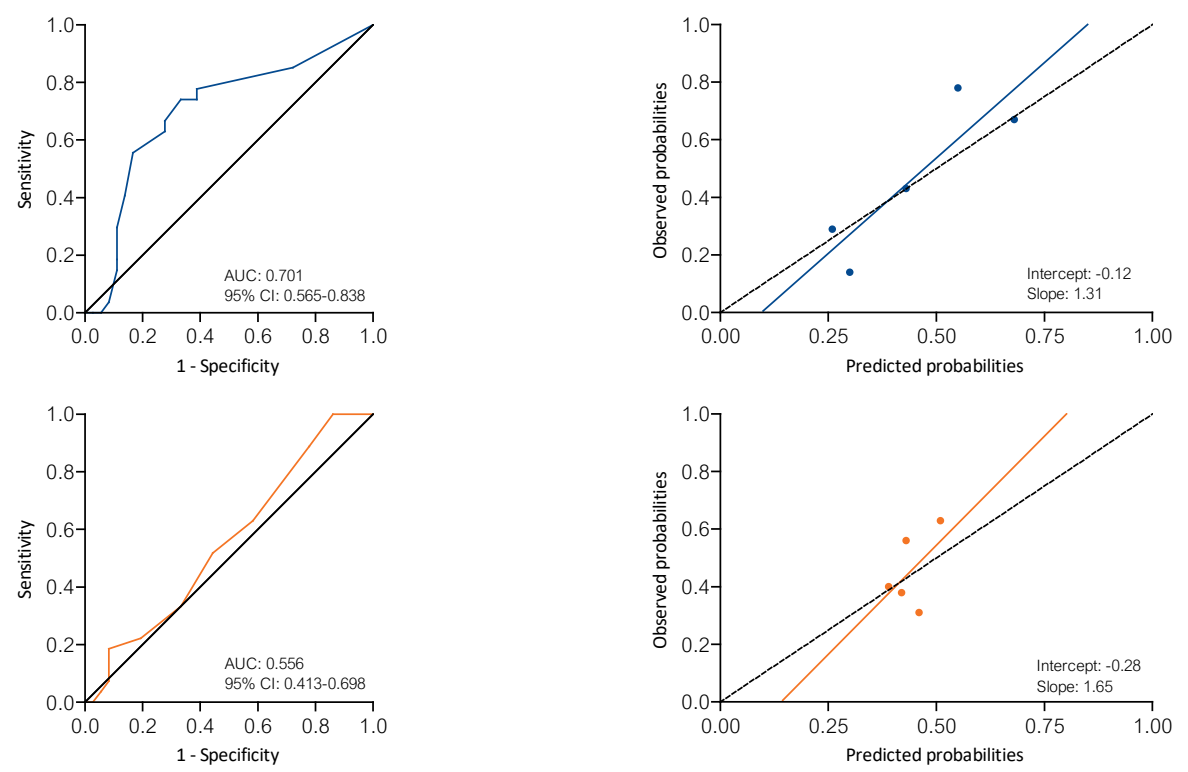

Figure 5.2. Performance of risk prediction models for major morbidity demonstrated by ROC curve and calibration curves for risk prediction model of Wiltberger et al. [9] (upper panel) and Kelly et al. [34] (lower panel).

Abbreviations: $\mathrm{AUC}=$ area under the curve; $\mathrm{Cl}=$ confidence interval; $\mathrm{ROC}=$ receiver operating characteristic. 


\section{Composite outcomes}

In total, 27 patients (42.9\%) developed a major complication, of which 22 patients (81.5\%) experienced a time to recovery of physical functioning above its median value $(n=19)$ or deceased $(n=3)$ (composite endpoint 1), whereas five of these patients $(18.5 \%)$ experienced a time to recovery of physical functioning below its median value (composite endpoint 2). When preoperative demographics and level of physical functioning data were clustered according to composite endpoints (see Table 5.4), a clinically relevant and statistically significant difference between both groups was observed in age (years), preoperative aerobic capacity (relative SRT performance, meaning the absolute SRT performance in Watt normalized for the patient's body mass $[\mathrm{W} / \mathrm{kg}]$ ), functional mobility (2MWT, in $\mathrm{m}$ ), and perceived level of functional capacity to perform activities of daily life (DASI, in METs) $(P<0.05)$. 
Table 5.4. Demographics and level of physical functioning of patients undergoing pancreatic resection with a higher (composite endpoint 1) and lower (composite endpoint 2) impact of a major complications

\begin{tabular}{|c|c|c|c|}
\hline & Composite endpoint $1^{a}$ & Composite endpoint $2^{b}$ & \multirow[b]{2}{*}{$P$} \\
\hline & Present $(n=22)$ & Present $(n=5)$ & \\
\hline \multicolumn{4}{|l|}{ Demographics } \\
\hline Sex ratio $(M: F)$ & $11: 11$ & $4: 1$ & 0.342 \\
\hline Age $\left(\right.$ years) ${ }^{c}$ & $73.0(26.0-85.0)$ & $61.0(56.0-72.0)$ & 0.026 \\
\hline$<70$ & $6(27.3)$ & $4(80.0)$ & 0.015 \\
\hline$\geq 70$ & $16(72.2)$ & $1(20.0)$ & \\
\hline BMI $\left(\mathrm{kg} / \mathrm{m}^{2}\right)^{\mathrm{c}}$ & $26.1(19.1-39.3)$ & $24.7(20.8-26.7)$ & 0.525 \\
\hline Normal (18.5-24.9) & $7(31.8)$ & $3(60.0)$ & \\
\hline Overweight (25.0-29.9) & $11(50.0)$ & $2(40.0)$ & \\
\hline Obese $(\geq 30.0)$ & $4(18.2)$ & $0(0.0)$ & \\
\hline ASA & & & 0.370 \\
\hline Grade I-II & $14(63.6)$ & $2(40.0)$ & \\
\hline Grade $\geq I I I$ & $8(36.4)$ & $3(60.0)$ & \\
\hline Comorbidities & & & 1.000 \\
\hline None & $3(13.6)$ & $1(20.0)$ & \\
\hline 1 comorbidity & $3(13.6)$ & $1(20.0)$ & \\
\hline$\geq 2$ comorbidities & $16(72.7)$ & $3(60.0)$ & \\
\hline Functional status & & & 0.636 \\
\hline Independent & $14(63.6)$ & $4(80.0)$ & \\
\hline Dependent & $8(36.4)$ & $1(20.0)$ & \\
\hline \multicolumn{4}{|l|}{ Physical functioning ${ }^{c}$} \\
\hline SRT (WR peak) & $160.0(67.0-301.00)$ & $201.0(162.0-239.0)$ & 0.062 \\
\hline SRT (WR & $2.07(0.92-3.13)$ & $2.89(1.87-4.12)$ & 0.047 \\
\hline HGS (kg) & $31.0(15.0-59.0)$ & $36.0(29.0-56.0)$ & 0.277 \\
\hline FTSTS test (s) & $8.91(4.78-28.93)$ & $6.94(5.44-14.07)$ & 0.314 \\
\hline TUG test (s) & $5.61(3.32-21.25)$ & $4.16(3.59-4.44)$ & 0.063 \\
\hline 2MWT (m) & $162.0(55.0-193.0)$ & $197.0(175.0-240.0)$ & 0.001 \\
\hline VSAQ (MET) & $5.5(3.0-11.0)$ & $9.0(8.0-11.0)$ & 0.091 \\
\hline DASI (MET) & $6.1(3.1-9.0)$ & $7.3(5.9-9.9)$ & 0.049 \\
\hline Complication grade & & & 0.255 \\
\hline Grade IIla & $9(40.9)$ & $3(60.0)$ & \\
\hline Grade IIIb & $0(0.0)$ & $1(20.0)$ & \\
\hline Grade IV & $10(45.5)$ & $1(20.0)$ & \\
\hline Grade V & $3(13.6)$ & $0(0.0)$ & \\
\hline
\end{tabular}

Abbreviations: ASA=American Society of Anesthesiologists; $\mathrm{BMI}=$ body mass index; $\mathrm{DASI}=$ Duke activity status index; FTSTS=five times sit-to-stand; HGS=handgrip strength; MET=metabolic equivalent of task; SRT=steep ramp test; TUG=timed up-and-go; VSAQ=veterans-specific activity questionnaire; $W_{\text {peak }}=$ peak work rate; $2 \mathrm{MWT}=$ two-minute walk test.

a: Major complication and a time to recovery of physical functioning above its median value ( $\geq 7$ days, higher impact).

b: Major complication and a time to recovery of physical functioning below its median value ( $\leq 6$ days, lower impact).

c: Values are expressed as median (range). 


\section{DISCUSSION}

In this study, we found that conventional factors (ASA grade III and platelet count) were associated with developing major complications, whereas aerobic capacity (SRT performance) was associated with time to recovery of physical functioning. External validation of two available preoperative prediction models for the risk of major complications demonstrated moderate-togood ability to discriminate between individuals with and without a major complication. However, these prediction models underestimated the risk for major complications in the current study population. Explorative analysis demonstrated that age and preoperative physical functioning might be related with the impact of a major complication for a patient.

The current study confirms that preoperative tests of physical functioning (SRT and 2MWT) were associated with time to recovery of physical functioning (see Table 5.2). This is congruent with other studies evaluating the relation between preoperative performance-based tests of physical functioning and postoperative time to recovery of physical functioning in similar patient populations [23, 24]. Conventional preoperative factors (ASA grade III and preoperative platelet count) were associated with the development of postoperative major complications (see Table 5.2). The presence of preoperative thrombocytosis (increased platelet count $>500 \times 10^{9} / \mathrm{L}$ ) has been shown to be associated with morbidity after distal pancreatectomy [34] and pancreaticoduodenectomy [8] in previous studies. Surprisingly, (estimated) aerobic capacity was not related with the development of a postoperative complication in the present study, which is not in line with findings from other studies [20-22]. The group without a major complication had a tendency to a higher absolute and relative preoperative WR $\mathrm{R}_{\text {peak }}$ at the SRT compared to the group who experienced a major complication (Table 5.2). One possible explanation could be the type of test that was used to estimate aerobic capacity: a SRT instead of a cardiopulmonary exercise test (the gold standard). Although, we did not find the same association between preoperative performance-tests of physical functioning and major complications as established in other studies $[25,45]$. A post-hoc analysis was performed to evaluate differences in postoperative outcomes between patients who underwent pancreaticoduodenectomy $(n=47)$ and patients who underwent distal pancreatectomy $(n=13)$. By doing so differences were found in time to recovery of physical functioning ( 7.5 versus 4.0 days, respectively; $P=0.055)$, complication incidence $(78.7 \%$ versus $46.2 \%$, respectively; $P=0.035)$, major complications $(48.9 \%$ versus $23.1 \%$, respectively; $P=0.122$ ), and length of hospital stay ( 14.5 days versus 9.0 days, respectively; $P=0.049$ ). In future observational studies these findings should be taken into account, preferably already in the setup of the studies in order to have sufficient power to introduce different types of surgery in the 
analysis. Furthermore, it is also of interest to study the association between the preoperative (natural) course (change scores) of physical fitness and physical activity level of the patient (improved, stable, declined) and postoperative outcomes, or to assess the effect of a preoperative exercise intervention combined with nutritional support.

A limited number of useful clinical models is currently available in the literature to predict major complications in patients undergoing pancreatic resection (Appendix 5.2, Table 5A.3). Performance of prediction models is often lower in the external validation cohort compared to the cohort in which the prediction model was developed [46]. This might be attributed to 1) the context in which the risk-prediction model is developed and validated (e.g. hospital-specific care pathways and protocols), 2) heterogeneity in defining (major) complications (Clavien-Dindo $\geq$ III) in the different prediction models [9,34],3) the type of surgery for which the risk-prediction model was developed, and 4) methodology and sample size considerations (e.g. the impact of sample size and the number of events on the performance of prognostic models [47]). The model of Kelly et al.[34] was externally validated on all types of pancreatic surgery in the current study cohort, whereas it was developed merely for patients undergoing a distal pancreatectomy. The latter could be a possible explanation of its low predictive performance in the present cohort. The usefulness of the model of Wiltberger et al. [9] in daily clinical practice was further evaluated in a post-hoc analysis. The positive predictive value was $64.3 \%$ and the negative predicted value was $74.3 \%$. The positive predictive value is relatively low; thus, only a small proportion of those considered at risk actually develop complications. Available models in the literature merely contain conventional predictors (e.g. age, comorbidities) for postoperative major complications. Predictors related to preoperative physical functioning (e.g. aerobic capacity) should be added to further improve the accuracy of preoperative risk stratification. Furthermore, the assessment of muscle mass with the use of computed tomography in combination with performance-based tests may provide additive risk prediction accuracy and the ability to develop targeted preoperative interventions [48].

Concerning the impact of a major complication, its relation with time to recovery of physical functioning was explored. There was an independent statistically significant difference between age and level of physical functioning between patients who experienced a major complication with a delayed recovery of physical functioning compared to patients who experienced a major complication without a delayed recovery of physical functioning (see Table 5.4). Patients who were younger, patients with a higher preoperative level of physical functioning (SRT and 2WMT performances), and patients with a higher perceived level of functional capacity to perform 
activities of daily life (DASI) were more likely to better cope with a major postoperative complication. Present study results are in line with a post-hoc analysis from Hulzebos et al. [49]. The study reported that patients who received preoperative inspiratory muscle training seemed to cope better with postoperative pneumonia compared to patients in the usual care-group (without this type of training), as their median hospitalization was shorter (11.5 versus 13.0 days, respectively) [49]. Hence, fitter patients might be more resistant and might cope better with the negative effects of postoperative complications. However, results in the current study showed a large range in preoperative physical functioning in patients experiencing major complications that had a time to recovery of physical functioning above its median value in the current study cohort. Nevertheless, it is hypothesized that a postoperative complication has less impact in fitter patients. Further research is required to investigate this hypothesis, as well as to look for alternatives outcome measures for evaluating the impact of a postoperative complication.

In conclusion, preoperative prediction of postoperative outcomes is feasible but needs another approach, as existing risk prediction models to predict major complications in patients undergoing major pancreatic surgery may have a limited role in clinical practice given their predictive performance. Only conventional predictors are used in currently available models and the current study shows that there might be room for improvement by also including variables of preoperative physical functioning. Furthermore, it is debatable whether optimal outcome measures are used by evaluating the incidence of complications without taking its impact into account.

\section{ACKNOWLEDGEMENTS}

We would like to thank Lieke Corpelijn and Nicole Haesen (Department of Surgery, Maastricht $\mathrm{UMC}+$ ) for guiding and referring patients to the Department of Physical Therapy. Furthermore, Nynke Westra for entering data into the database. 


\section{REFERENCES}

1. Fearon KC, Voss AC, Hustead DS; Cancer Cachexia Study Group. Definition of cancer cachexia: effect of weight loss, reduced food intake, and systemic inflammation on functional status and prognosis. Am J Clin Nutr. 2006;83:1345-50.

2. Gouma DJ, van Geenen RC, van Gulik TM, de Haan RJ, de Wit LT, Busch OR, et al. Rates of complications and death after pancreaticoduodenectomy: risk factors and the impact of hospital volume. Ann Surg. 2000 232:786-95

3. Büchler MW, Wagner M, Schmied BM, Uhl W, Friess $\mathrm{H}, \mathrm{Z}^{\prime}$ graggen $\mathrm{K}$. Changes in morbidity after pancreatic resection: toward the end of completion pancreatectomy. Arch Surg. 2003;138:1310-4

4. Dutch Institute for Clinical Auditing (DICA) Leiden. DICA jaarrapportage 2017; DPCA. 2017. Ascessed november 2018, http://dica.nl/jaarrapportage-2017.

5. Cooper AB, Tzeng CW, Katz MH. Treatment of borderline resectable pancreatic cancer. Curr Treat Options Oncol. 2013;14:293-310.

6. Silver JK, Baima J. Cancer prehabilitation: an opportunity to decrease treatmentrelated morbidity, increase cancer treatment options, and improve physical and psychological health outcomes. Am J Phys Med Rehabil. 2013;92:715-27.

7. Venkat R, Puhan MA, Schulick RD, Cameron JL, Eckhauser FE, Choti MA, et al. Predicting the risk of perioperative mortality in patients undergoing pancreaticoduodenectomy: a novel scoring system. Arch Surg. 2011;146:1277-84.

8. Greenblatt DY, Kelly KJ, Rajamanickam V, Wan Y, Hanson T, Rettammel R, et al. Preoperative factors predict perioperative morbidity and mortality after pancreaticoduodenectomy. Ann Surg Oncol. 2011;18:2126-35.

9. Wiltberger G, Muhl B, Benzing C, Atanasov G, Hau HM, Horn M, et al. Preoperative risk stratification for major complications following pancreaticoduodenectomy: Identification of high-risk patients. Int J Surg. 2016;31:33-9.
10. Pratt $\mathrm{W}$, Joseph $\mathrm{S}$, Callery MP, Vollmer CM Jr. POSSUM accurately predicts morbidity for pancreatic resection. Surgery. 2008;143:8-19.

11. Bourgouin S, Ewald J, Mancini J, Moutardier V, Delpero JR, Le Treut YP. Predictive factors of severe complications for ampullary, bile duct and duodenal cancers following pancreaticoduodenectomy: Multivariate analysis of a 10-year multicentre retrospective series. Surgeon. 2017;15:251-58

12. Jang M, Park HW, Huh J, Lee JH, Jeong YK, Nah YW, Park J, Kim. Predictive value of sarcopenia and visceral obesity for postoperative pancreatic fistula after pancreaticoduodenectomy analyzed on clinically acquired CT and MRI. Eur Radiol. 2018;7.

13. Hu BY, Wan T, Zhang WZ, Dong JH. Risk factors for postoperative pancreatic fistula: Analysis of 539 successive cases of pancreaticoduodenectomy. World J Gastroenterol. 2016;14:7797-805.

14. Samim M, Mungroop TH, AbuHilal M, Isfordink CJ, Molenaar QI, van der Poel MJ, et al. Surgeons' assessment versus risk models for predicting complications of hepato-pancreato-biliary surgery (HPBRISC): a multicenter prospective cohort study. HPB (Oxford). 2018;20:809-14.

15. Van Beijsterveld CA, Heldens AF, Bongers $B C$, Van Meeteren. A survey of a current clinical practice in Dutch hospitals: reported pre-and postoperative physical therapy management for patients opting for elective major abdominal surgery. Journal of physical therapy. 2019;99:1291-303.

16. Hoogeboom TJ, Dronkers JJ, Hulzebos $\mathrm{EH}$, Van Meeteren. Merits of exercise therapy before and after major surgery. Curr Opin Anaesthesiol. 2014;27:161-6.

17. Desborough JP. The stress response to trauma and surgery. Br J Anaesth. 2000;85:109-17. 
18. Finnerty CC, Mabvuure NT, Ali A, Kozar RA, Herndon DN. The surgically induced stress response. J Parenter Enteral Nutr. 2013;37:21S-9S.

19. Dronkers J, Witteman B, van Meeteren N. Surgery and functional mobility: doing the right thing at the right time. Tech Coloproctol. 2016;20:339-41.

20. Ausania F, Snowden CP, Prentis JM, Holmes LR, Jaques BC, White SA, French $\mathrm{JJ}$, et al. Effects of low cardiopulmonary reserve on pancreatic leak following pancreaticoduodenectomy. Br J Surg. 2012;99:1290-4.

21. Junejo MA, Mason JM, Sheen AJ, Bryan A, Moore J, Foster P et al. Cardiopulmonary exercise testing for preoperative risk assessment before pancreaticoduodenectomy for cancer. Ann Surg Oncol. 2014;21:1929-36.

22. Chandrabalan VV, McMillan DC, Carter R, Kinsella J, McKay CJ, Carter CR et al. Preoperative cardiopulmonary exercise testing predicts adverse post-operative events and non-progression to adjuvant therapy after major pancreatic surgery. HPB (Oxford). 2013;15:899-907.

23. Heldens AFJM, Bongers BC, Lenssen AF, Stassen LPS, Buhre WF, van Meeteren NLU. The association between performance parameters of physical fitness and postoperative outcomes in patients undergoing colorectal surgery: An evaluation of care data. Eur J Surg Oncol. 2017;43:2084-92.

24. Dronkers JJ, Chorus AM, van Meeteren NL, Hopman-Rock M. The association of pre-operative physical fitness and physical activity with outcome after scheduled major abdominal surgery. Anaesthesia. 2013;68:67-73.

25. Hayashi K, Yokoyama Y, Nakajima H, Nagino M, Inoue T, Nagaya M, Hattori K, et al. Preoperative 6-minute walk distance accurately predicts postoperative complications after operations for hepatopancreato-biliary cancer. Surgery. 2017;161:525-32.
26. Moran J, Guinan E, McCormick P, Larkin J, Mockler D, Hussey $\mathrm{J}$ et al. The ability of prehabilitation to influence postoperative outcome after intra-abdominal operation: A systematic review and meta-analysis.

Surgery. 2016;160:1189-201.

27. Santa Mina D, Clarke H, Ritvo P, Leung YW, Matthew AG, Katz J, et al. Effect of total-body prehabilitation on postoperative outcomes: a systematic review and metaanalysis. Physiotherapy. 2014;100:196207.

28. van der Leeden M, Huijsmans R, Geleijn E, de Lange-de Klerk ES, Dekker J, Bonjer $\mathrm{HJ}$, et al. Early enforced mobilisation following surgery for gastrointestinal cancer: feasibility and outcomes. Physiotherapy. 2016;102:103-10.

29. Yip VS, Dunne DF, Samuels S, Tan CY, Lacasia C, Tang J, et al. Adherence to early mobilisation: Key for successful enhanced recovery after liver resection. Eur J Surg Oncol. 2016;42:1561-7.

30. Reeve JC, Boden I. The physiotherapy management of patients undergoing abdominal surgery. New Zealand Journal of Physiotherapy, 2016;44.

31. Shields RK, Enloe LJ, Evans RE, Smith KB, Steckel SD. Reliability, validity, and responsiveness of functional tests in patients with total joint replacement. Phys Ther. 1995;75:169-76

32. Elings J, Zoethout S, Ten Klooster PM, van der Sluis G, van Gaalen SM, van Meeteren NLU, et al. Advocacy for use of the modified lowa Level of Assistance Scale for clinical use in patients after hip replacement: an observational study. Physiotherapy. 2018;27:S00319406(18)30153-6.

33. Oken MM, Creech RH, Tormey DC, Horton J, Davis TE, McFadden ET, et al. Toxicity and response criteria of the Eastern Cooperative Oncology Group. Am J Clin Oncol. 1982;5:649-55.

34. Kelly KJ, Greenblatt DY, Wan Y, Rettammel RJ, Winslow E, Cho CS, et al. Risk stratification for distal pancreatectomy utilizing ACS-NSQIP: preoperative factors predict morbidity and mortality. J Gastrointest Surg. 2011;15:250-9. 
35. Kneuertz PJ, Pitt HA, Bilimoria KY, Smiley JP, Cohen ME, Ko CY, et al. Risk of morbidity and mortality following hepatopancreato-biliary surgery. J Gastrointest Surg. 2012;16:1727-35.

36. Aoki S, Miyata H, Konno H, Gotoh M, Motoi $\mathrm{F}$, Kumamaru $\mathrm{H}$, et al. Risk factors of serious postoperative complications after pancreaticoduodenectomy and risk calculators for predicting postoperative complications: a nationwide study of 17,564 patients in Japan. J Hepatobiliary Pancreat Sci. 2017;24:243-51.

37. Bassi C, Marchegiani G, Dervenis C, Sarr M, Abu Hilal M, Adham M, et al. The 2016 update of the International Study Group (ISGPS) definition and grading of postoperative pancreatic fistula: 11 Years After. Surgery. 2017;161:584-91.

38. Wente MN, Bassi C, Dervenis C, Fingerhut A, Gouma DJ, Izbicki JR, et al. Delayed gastric emptying (DGE) after pancreatic surgery: a suggested definition by the International Study Group of Pancreatic Surgery (ISGPS). Surgery. 2007;142:7618.

39. Wente MN, Veit JA, Bassi C, Dervenis C, Fingerhut A, Gouma DJ, et al.

Postpancreatectomy hemorrhage (PPH): an International Study Group of Pancreatic Surgery (ISGPS) definition. Surgery. 2007;142:20-5.

40. Besselink MG, van Rijssen LB, Bassi C, Dervenis C, Montorsi M, Adham M, et al. Definition and classification of chyle leak after pancreatic operation: A consensus statement by the International Study Group on Pancreatic Surgery. Surgery. 2017;161:365-72.

41. Koch M, Garden OJ, Padbury R, Rahbari NN, Adam R, Capussotti L, et al. Bile leakage after hepatobiliary and pancreatic surgery: a definition and grading of severity by the International Study Group of Liver Surgery. Surgery. 2011;149:680-8.
42. Dindo D, Demartines N, Clavien PA. Classification of surgical complications: a new proposal with evaluation in a cohort of 6336 patients and results of a survey. Ann Surg. 2004;240:205-13.

43. Collins GS, Reitsma JB, Altman DG, Moons KG. Transparent reporting of a multivariable prediction model for individual prognosis or diagnosis (TRIPOD):the TRIPOD statement. BMJ. 2015;7.

44. Altman DG, Vergouwe $Y$, Royston P, Moons KG. Prognosis and prognostic research: validating a prognostic model. BMJ. 2009;28.

45. Karlsson E, Egenvall M, Farahnak $\mathrm{P}$, Bergenmar M, Nygren-Bonnier M, Franzén $E$, et al. Better preoperative physical performance reduces the odds of complication severity and discharge to care facility after abdominal cancer resection in people over the age of 70 - $\mathrm{A}$ prospective cohort study. Eur J Surg Oncol. 2018;44:1760-7.

46. Moons KG, Kengne AP, Grobbee DE, Royston P, Vergouwe Y, Altman DG, et al. Risk prediction models: II. External validation, model updating, and impact assessment. Heart. 2012;98:691-8.

47. Collins GS, Ogundimu EO, Altman DG. Sample size considerations for the external validation of a multivariable prognostic model: a resampling study. Stat Med. 2016;35:214-26.

48. van Dijk DP, Bakens MJ, Coolsen MM, Rensen SS, van Dam RM, Bours MJ, et al. Low skeletal muscle radiation attenuation and visceral adiposity are associated with overall survival and surgical site infections in patients with pancreatic cancer. J Cachexia Sarcopenia Muscle. 2017;8(2):317-26.

49. Hulzebos EH, Helders PJ, Favié NJ, De Bie RA, Brutel de la Riviere A, Van Meeteren NL. Preoperative intensive inspiratory muscle training to prevent postoperative pulmonary complications in high-risk patients undergoing CABG surgery: a randomized clinical trial. JAMA. 2006;296:1851-7. 
APPENDIX

Appendix 5.1.

Patient journey

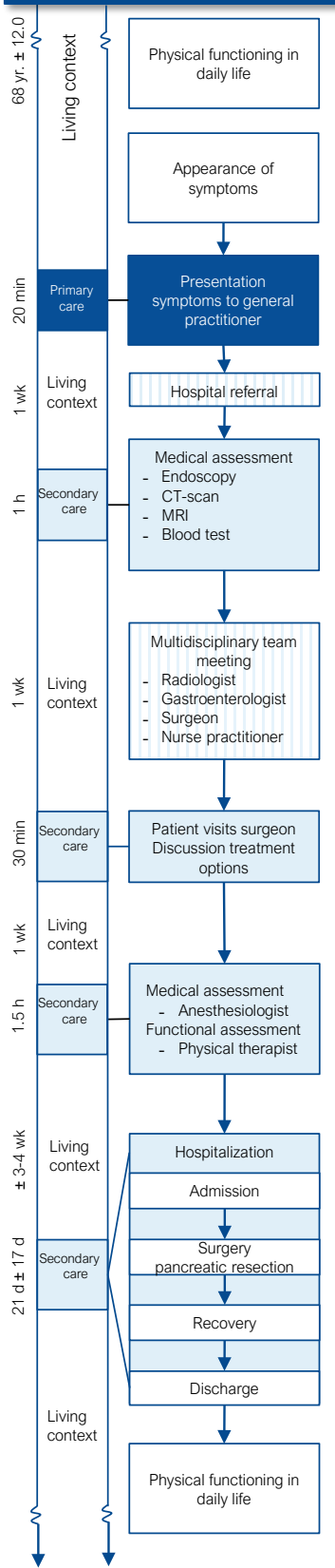

Figure 5A.1. Overview of the patient journey 


\section{Preoperative screening of physical functioning}

The preoperative screening by an experienced hospital physical therapist evaluated the patient's preoperative performance and perception of physical functioning by measuring their aerobic capacity, muscle strength, functional mobility, and perceived level of functional capacity to perform activities of daily living (ADL). The physical activity readiness questionnaire [1] was filled out to assess whether there were contra-indications for exercise testing.

\section{Aerobic capacity}

The steep ramp test (SRT), a short-time maximal exercise test on a cycle ergometer up to maximal exertion, was used to estimate the patient's aerobic capacity. To make the SRT suitable for our surgical population, the original protocol (work rate increments of $25 \mathrm{~W}$ each $10 \mathrm{~s}$ ) was modified [2]. The test was performed with the use of a calibrated cycle ergometer (Lode Corival Rehab, Lode BV, Groningen, the Netherlands). Patients wore a pulse oximeter and a Polar (T31i transmitter, Polar, Kempele, Finland) heart rate belt. Seat height was set at comfortable height for each individual. After two minutes of unloaded cycling, the work rate was increased by constant increments of 10 Watt each $10 \mathrm{~s}$ in a ramp like manner. Throughout the test, patients were instructed to keep the pedaling at a frequency around 80 revolutions $/ \mathrm{min}$. The protocol continued until the patient's pedaling frequency fell definitely below 60 revolutions/min despite strong verbal encouragement. Peak work rate $\left(W_{R_{\text {peak }}}\right.$ in Watts), peripherally measured oxygen saturation (\%), heart rate (beats/min), perceived level of exertion (0-10 BORG scale), and stop

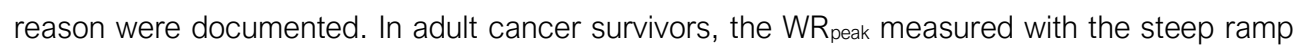
test was found to be highly correlated with peak oxygen uptake $\left(\mathrm{Vo}_{2 \text { peak }}\right)$ achieved during a cardiopulmonary exercise test ( $r$ of 0.82), and the SRT was found to be a reliable test (intra-class correlation coefficient [ICC] for the achieved WR $\mathrm{R}_{\text {peak }}$ of 0.996) [3].

\section{Muscle strength}

General muscle strength was measured by handgrip strength (HGS), using a hand-held dynamometer (JAMAR® Hydraulic Hand Dynamometer, JAMAR, Patterson Medical Holdings, Inc., Illinois, USA). Patients were measured while seated at a chair (height $43-47 \mathrm{~cm}$ ) with no arm support, the shoulder adducted, elbow flexed at 90 degrees, and the forearm in neutral position. The instruction was to maximally use pinch strength with the patient's dominant hand and verbal encouragement of the physical therapist. The test was repeated three times, of which the highest score (in $\mathrm{kg}$ ) was reported. The HGS measurement has shown a good test-retest reliability (ICC of 0.94 in healthy adults) [4]. 


\section{Functional mobility}

Functional mobility was evaluated using the five times sit-to-stand (FTSTS) test (in s), timed upand-go (TUG) test (in s), and the two-minute walk test (2MWT, in $\mathrm{m}$ ). The FTSTS test evaluates a combination of functional mobility, balance, and lower leg muscle strength $[5,6]$ and is considered reliable in community-dwelling older people (ICC of 0.89 ; $95 \%$ confidence interval [Cl] of 0.79 to 0.95 ) [6]. The test measures the time taken to stand five times from a sitting position with crossed arms (chair height $45-47 \mathrm{~cm}$ ) as fast as possible, measured with a stopwatch. Timing started on 'start' and ended when the fifth stand was completed. The TUG test recorded the time in seconds needed to rise from an arm chair ( 43 to $47 \mathrm{~cm}$ in height), walk three meters, return to the chair, and sit down again, all as quickly as possible [7]. If necessary patients were allowed to use their regulatory walking aid. In patients undergoing colorectal cancer surgery, the TUG test is associated with time to recovery of physical functioning (odds ratio [OR] of 1.274; $95 \% \mathrm{Cl}$ of 0.975 to $1.664 ; P=0.008$ ) [8]. Furthermore, the TUG test in geriatric patients has been shown to have an excellent inter-rater (ICC of 0.99) and intra-rater (ICC of 0.99) reliability [7]. The 2MWT records the distance (in $\mathrm{m}$ ) that is walked over a length of 15 meters in two minutes, if applicable with a customary walking aid. The 2MWT is correlated with the twelve-minute walk test ( $r$ of 0.864) and the six-minute walk test ( $r$ of 0.892) in a small sample of patients with respiratory disease [9]. In community-dwelling healthy adults (18-85) this test has shown to have good test-retest reliability (ICC of $0.82 ; 95 \% \mathrm{Cl}$ of 0.76 to 0.87 ) [10].

\section{Perceived level of physical functional capacity}

Two questionnaires were used to gain insight in the patient's perceived level of functional capacity to perform ADL. The veterans-specific activity questionnaire (VSAQ) consists of thirteen (groups of) activities listed in a progressive order concerning their intensity. The activities are linked to a metabolic equivalent of task (MET) score and patients have to report the activities they are able to perform without the presence of symptoms. A line is drawn below those (groups of) activities and the corresponding MET score is reported. Among healthy older adults (66-86 years), the VSAQ was found to have a good inter-rater reliability (ICC of 0.882 ) to assess functional capacity to perform ADL [11]. The Duke activity status index (DASI) consists of twelve items related to the patient's ADL. Based on the weighted sum scores on each item a prediction of the patient's VO2peak can be made. The questionnaire has been shown to be a sensitive and specific predictor for VAT $\geq 11 \mathrm{~mL} / \mathrm{kg} / \mathrm{min}$ (AUC of $0.767 ; 95 \% \mathrm{Cl}$ of 0.630 to $0.994 P=0.003$ ) but it has only a moderate correlation ( $r$ of $0.67, P<0.0001$ ) with $V_{0}$ 2peak (in $\mathrm{mL} / \mathrm{kg} / \mathrm{min}$ ) among patients undergoing elective intra-abdominal surgery [12]. 


\section{References}

1. Thomas S, Reading J, Shephard RJ. Revision of the physical activity readiness Questionnaire (PAR-Q). Can J Sport Sci. 1992;17:338-45.

2. Meyer K, Samek L, Schwaibold M, Westbrook S, Hajric R, Lehmann M, et al. Physical responses to different modes of interval exercise in patients with chronic heart failure--application to exercise training. Eur Heart J. 1996;17:1040-7.

3. De Backer IC, Schep G, Hoogeveen A, Vreugdenhil G, Kester AD, van Breda $\mathrm{E}$. Exercise testing and training in a cancer rehabilitation program: the advantage of the steep ramp test. Arch Phys Med Rehabil. 2007;88:610-6.

4. Kamimura T, Ikuta Y. Evaluation of grip strength with a sustained maximal isometric contraction for 6 and 10 seconds. J Rehabil Med. 2001;33:225-9.

5. Lord SR, Murray SM, Chapman K, Munro $B$, Tiedemann A. Sit-to-stand performance depends on sensation, speed, balance, and psychological status in addition to strength in older people. J Gerontol A Biol Sci Med Sci. 2002;57:M539-43.

6. Tiedemann A, Shimada $\mathrm{H}$, Sherrington $\mathrm{C}$, Murray S, Lord S. The comparative ability of eight functional mobility tests for predicting falls in community-dwelling older people. Age Ageing. 2008;37:430-5.

7. Podsiadlo D, Richardson S. The timed "Up \& Go": a test of basic functional mobility for frail elderly persons. J Am Geriatr Soc. 1991;39:142-8.
8. Heldens AFJM, Bongers BC, Lenssen AF, Stassen LPS, Buhre WF, van Meeteren NLU. The association between performance parameters of physical fitness and postoperative outcomes in patients undergoing colorectal surgery: An evaluation of care data. Eur J Surg Oncol. 2017;43:2084-92.

9. Pang J, Gross ER, Woodcock AA, Woodcock AA, Geddes DMI. Two-, six-, and 12-minute walking tests in respiratory disease. Br Med J (Clin Res Ed). 1982;284:1607-8.

10. Bohannon RW, Wang YC, Gershon RC Two-minute walk test performance by adults 18 to 85 years: normative values, reliability, and responsiveness. Arch Phys Med Rehabil. 2015;96:472-7.

11. de Carvalho Bastone A, de Souza Moreira B, Teixeira CP, Dias JM, Dias RC. Is the Veterans Specific Activity Questionnaire Valid to Assess Older Adults Aerobic Fitness? J Geriatr Phys Ther. 2016;39:11724.

12. Struthers R, Erasmus $P$, Holmes $K$, Warman P, Collingwood A, Sneyd JR. Assessing fitness for surgery: a comparison of questionnaire, incremental shuttle walk, and cardiopulmonary exercise testing in general surgical patients. $\mathrm{Br} \mathrm{J}$ Anaesth. 2008;101:774-80. 


\section{Appendix 5.2.}

Table 5A.2. Search strategy for the systematic review of the literature for cohort studies developing models to predict the risk for major complications following pancreatic resection published within the last ten years. Search performed in PubMed on October 22, 2018

\begin{tabular}{|c|c|c|}
\hline Search & Queries & Hits \\
\hline \#1 Pancreas & Search ("pancreas"[MeSH Terms] OR "pancreas"[All Fields]) & 179597 \\
\hline \#2 Cancer & $\begin{array}{l}\text { Search ("neoplasms"[MeSH Terms] OR "neoplasms"[All } \\
\text { Fields] OR "cancer"[All Fields]) }\end{array}$ & 3746857 \\
\hline \#3 Surgery & $\begin{array}{l}\text { Search ("surgery"[Subheading] OR "surgery"[All Fields] OR } \\
\text { "surgical procedures, operative"[MeSH Terms] OR } \\
\text { ("surgical"[All Fields] AND "procedures"[All Fields] AND } \\
\text { "operative"[All Fields]) OR "operative surgical procedures"[All } \\
\text { Fields] OR "surgery"[All Fields] OR "general surgery"[MeSH } \\
\text { Terms] OR ("general"[All Fields] AND "surgery"[All Fields]) } \\
\text { OR "general surgery"[All Fields]) OR } \\
\text { ("pancreaticoduodenectomy"[MeSH Terms] OR } \\
\text { "pancreaticoduodenectomy"[All Fields]) }\end{array}$ & 4365366 \\
\hline $\begin{array}{l}\# 4 \\
\text { Type of study }\end{array}$ & 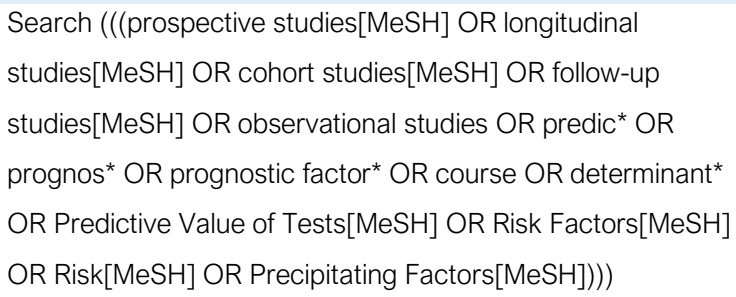 & 4561058 \\
\hline \#5 & $\begin{array}{l}\text { Search ("complications"[Subheading] OR "complications"[All } \\
\text { Fields]) OR ("epidemiology"[Subheading] OR } \\
\text { "epidemiology"[All Fields] OR "morbidity"[All Fields] OR } \\
\text { "morbidity"[MeSH Terms]) }\end{array}$ & 4823069 \\
\hline \#6 & \#1 AND \#2 AND \#3 AND \#4 AND \#5 & 5373 \\
\hline$\# 7$ & \#6 Filters: published in the last 10 years & 2599 \\
\hline
\end{tabular}




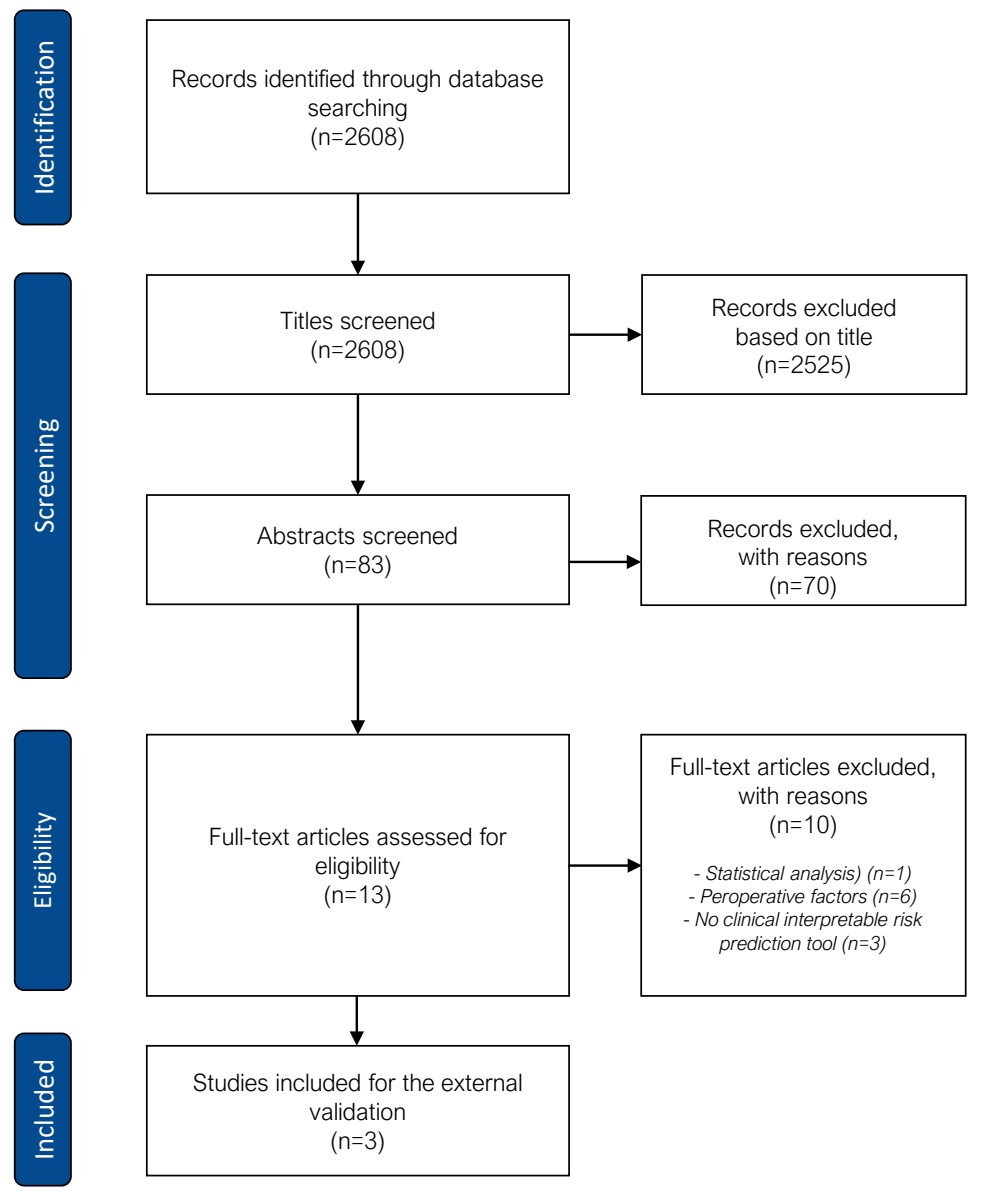

Figure 5A.2. Flow chart of included studies. 


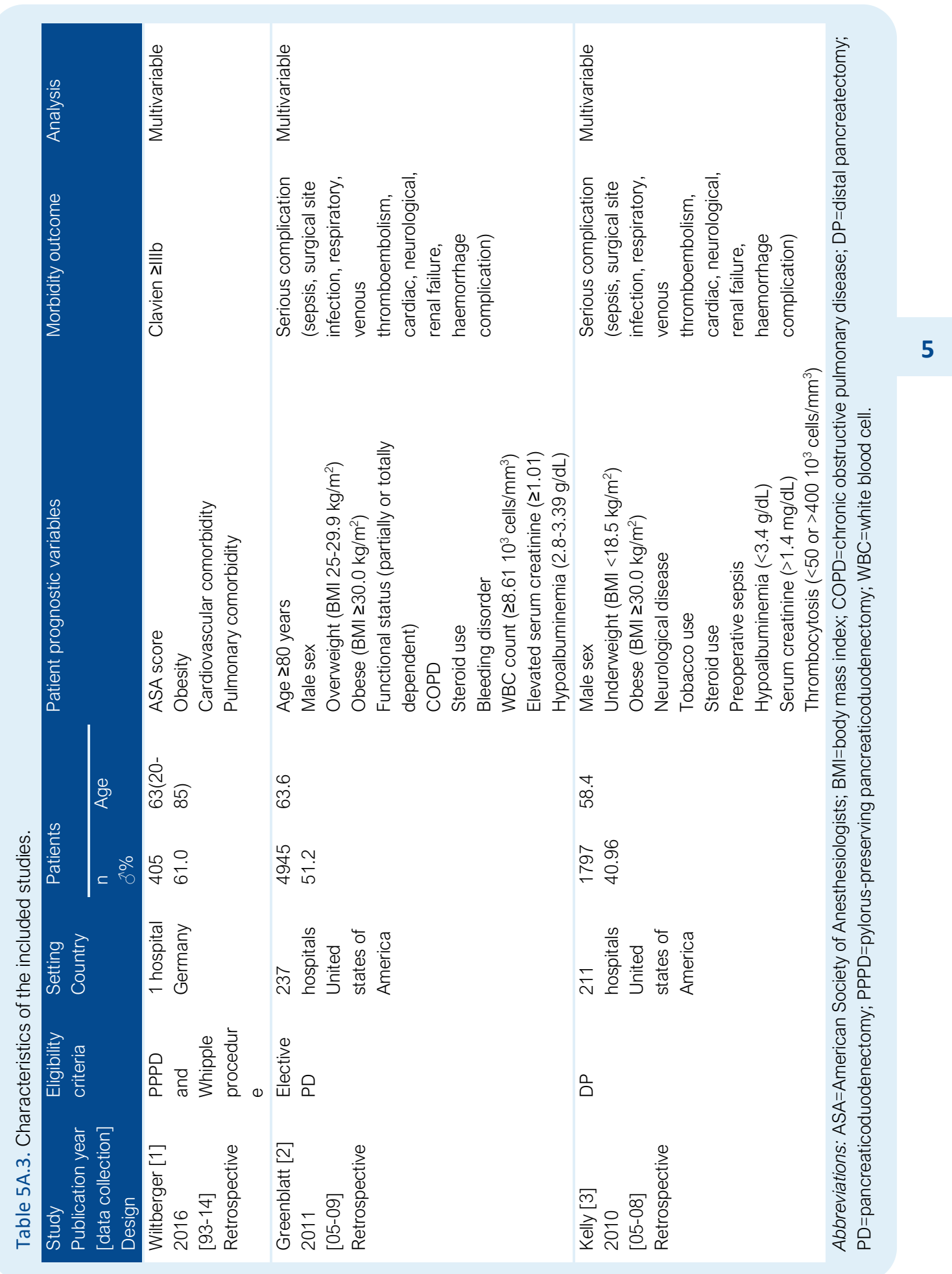


Table 5A.4. Preoperative variables included in the risk-prediction models.

\begin{tabular}{|c|c|c|c|}
\hline & Wiltberger [1] & Greenblatt [2] & Kelly [3] \\
\hline Age (years) & & + & \\
\hline Sex (male or female) & & + & + \\
\hline $\mathrm{BMI}\left(\mathrm{kg} / \mathrm{m}^{2}\right)$ & + & + & + \\
\hline ASA class & + & & \\
\hline \multicolumn{4}{|l|}{ Comorbidities } \\
\hline Cardiac & + & & \\
\hline Vascular & + & & \\
\hline Pulmonary & + & + & \\
\hline Neurological & & & + \\
\hline Bleeding disorder & & + & \\
\hline Functional status & & + & \\
\hline Tobacco use & & & + \\
\hline Steroid use & & + & + \\
\hline Preoperative sepsis & & & + \\
\hline \multicolumn{4}{|l|}{ Laboratory results } \\
\hline Platelet count & & & + \\
\hline WBC count & & + & \\
\hline Albumin level & & + & + \\
\hline Creatinine level & & + & + \\
\hline $\begin{array}{l}\text { Abbreviations: } \mathrm{ASA}= \\
\text { WBC=white blood cell }\end{array}$ & an Society of & sthesiologists; & body mass index; \\
\hline
\end{tabular}

\section{References}

1. Wiltberger G, Muhl B, Benzing C, Atanasov G, Hau HM, Horn M, et al. Preoperative risk stratification for major complications following pancreaticoduodenectomy: Identification of high-risk patients. Int J Surg. 2016;31:33-9.

2. Greenblatt DY, Kelly KJ, Rajamanickam V, Wan Y, Hanson T, Rettammel R, et al. Preoperative factors predict perioperative morbidity and mortality after pancreaticoduodenectomy. Ann Surg Oncol. 2011;18:2126-35.
3. Kelly KJ, Greenblatt DY, Wan Y, Rettammel RJ, Winslow E, Cho CS, et al. Risk stratification for distal pancreatectomy utilizing ACS-NSQIP: preoperative factors predict morbidity and mortality. J Gastrointest Surg. 2011;15:250-9. 
Preoperative physical functioning and the impact of a postoperative complication

NOTES 
Pas als er aandacht is voor de concrete context waarin het bewijs verkregen is, krijgt het bewijs betekenis (vrij naar RVS, 2016), zoals preoperatieve fysiotherapie pas écht betekenis krijgt als deze zich in de leefomgeving van de patiënt begeeft. 


\section{Chapter 6}

Personalized community-based prehabilitation in a highrisk surgical patient opting for pylorus-preserving pancreaticoduodenectomy: a case report

Christel A. van Beijsterveld | Bart C. Bongers | Marcel den Dulk | Cornelius H. Dejong | Nico L. van Meeteren 


\section{ABSTRACT}

\section{Introduction}

Prehabilitation aims for an optimal physical functioning level before, during, and after hospitalization for major surgery. The purpose of this case report was to illustrate the care pathway of a high-risk patient who opted for pylorus-preserving pancreaticoduodenectomy, including preparation for this procedure by participating in a community-based exercise prehabilitation program. The report describes patient examination, evaluation in decision-making for surgery, the prehabilitation program, and outcomes within the context of the HypothesisOriented Algorithm for Clinicians II.

\section{Case description}

The patient was a 75-year-old woman with a history of several comorbidities who was found to have a polypoid mass in the descending segment of the duodenum. Based on the preoperative assessment, the level of physical functioning was expected to be insufficient to cope adequately with the stress of hospitalization and surgery. Intervention: A four-week prehabilitation program, including aerobic, resistance, and functional task training in a community-based physical therapy practice.

\section{Outcomes}

Prehabilitation had a beneficial impact on improving functional mobility preoperatively (timed upand-go test score from 19.41 to $10.03 \mathrm{~s}$, five times sit-to-stand test score from 30.05 to $10.05 \mathrm{~s}$, and 2-minute walk test distance from 55.0 to $107.0 \mathrm{~m}$ ). Surgery and postoperative recovery proceeded without complications. She achieved independent physical functioning on postoperative day 6 and was discharged home on postoperative day 12 .

\section{Conclusion}

Preoperative risk-assessment can support clinical decision-making in a high-risk patient opting for major abdominal surgery. Furthermore, a remarkable improvement in physical functioning can be achieved by prehabilitation in a high-risk patient. 


\section{INTRODUCTION}

Surgical and anesthetic techniques have improved significantly in the last decades, trying to minimize loss of a patient's (medical) condition during surgery. Still, the challenge remains to optimize the perioperative period and context, especially for frail patients who are at increased risk for delayed or permanent loss of physical functioning before, during, and after hospitalization and surgery [1-3]. Their increased risks can be counteracted by preoperatively challenging homeostatic and specifically allostatic mechanisms by supportive preoperative interventions (prehabilitation), enabling the patient to deal with the stressors of (the diagnosis of) their pathology, the preoperative 'waiting' period, hospital stay, and surgical intervention [4, 5]. Modifiable patient factors, such as aerobic capacity, level of physical functioning, and nutritional status may determine the capacity of the patient's homeostatic and allostatic responses to these stressors and consequently their postoperative recovery [6].

Prehabilitation is advocated for patients with a high-risk profile. Hulzebos and Van Meeteren [5] described prehabilitation within a proactive perioperative care strategy aiming for an optimal level of physical functioning before, during, and after hospitalization, inspired by Hood's P4-medicine approach $[5,7]$. Perioperative care would best be 1) predictive (e.g., preoperatively screening a patient's individual risk factors), 2) preventive (e.g., offering preoperative interventions as physical exercise training, nutritional, and/or psychological support), 3) personalized (e.g., tailored inventions for the individual patient), and 4) participatory (e.g., encouraging the patient to actively participate in their treatment through active self-care and self-management) [8].

Considering the importance of proactive perioperative management in relation to postoperative outcomes, the purpose of this case report was to illustrate the care pathway of a high-risk patient who opted for pylorus-preserving pancreaticoduodenectomy (PPPD), including preparation for this procedure by participating in a community-based prehabilitation program. The role of the patient and physical therapist, both part of the interdisciplinary team, in managing perioperative care in major abdominal cancer surgery is described with the application of the HypothesisOriented Algorithm for Clinicians ॥ (HOAC I) for clinical reasoning [9]. The HOAC ॥ was combined with the ontology of the International Classification of Functioning (ICF), using the recently advised change of the WHO framework [10]. This case report followed the CARE guidelines [11] for case reports and evaluated therapeutic validity using the CONTENT scale [12]. 


\section{CASE DESCRIPTION: PATIENT HISTORY AND SYSTEM REVIEW}

This case report is about a 75-year-old woman, living alone in a senior apartment and able to perform her activities of daily living (ADL) independently. Her weekly physical activity consisted of walking to the supermarket $(75 \mathrm{~m})$. She would have preferred to be able to walk longer distances and in the past she enjoyed recreational swimming (one hour a week); however, because of complaints of dyspnea, probably due to her chronic obstructive pulmonary disease (COPD; GOLD II), she was not able to perform the latter activities anymore. For household cleaning activities, she had a cleaner once a week. She has one daughter and three grandchildren. Her medical history included right sided total hip replacement and revision, uterus extirpation, appendectomy, COPD (GOLD II, Tiffeneau index of 69\%), atrial fibrillation, left ventricular hypertrophy (left ventricular ejection fraction 50-55\%) and moderate stage $3 \mathrm{~A}$ renal failure (glomerular filtration rate $47 \mathrm{~mL} / \mathrm{min}$ ). She consulted her general practitioner with discomfort and the sensation of a lump behind her sternum after eating, as well as with itching complaints since six months. The lump behind her sternum was not painful and by drinking water, the discomfort disappeared. Her general practitioner referred her to the nearest general hospital for a gastroscopy and computed tomography (CT) scan. The CT scan of the abdomen showed a dilatation of the intrahepatic bile duct and the common bile duct with a polypoid mass in the descending segment (D2) of the duodenum. No lymphadenopathy or distant metastases were detected by the CT scan of the abdomen and thorax. A gastroduodenal endoscopy revealed a polyp in D2, occupying one-third of the lumen with probably the involvement of the ampulla of Vater. Pathological examination of the biopsy of the polyp revealed low-grade dysplasia without malignancy. After the diagnostic tests, the gastroenterologist explained that her esophagus and stomach were well; however, they discovered a large suspicious mass in her duodenum. The immediate reaction of the patient was: "That's like the ground falls out from beneath your feet! I was shocked." She was referred to the outpatient hepato-pancreato-biliary (HPB) unit of Maastricht University Medical Center+ to discuss her treatment options. In Figure 6.1, the patient journey is presented, ordered on a timeline starting from the time of her complaints. 


\section{Qualitative and quantitative clinical impression I}

During the multidisciplinary team meeting with the surgeon, gastroenterologist, radiologist, oncologist, and clinical nurse specialist, the results of the diagnostics tests (CT-scan, endoscopy, and pathology) and the treatment options for the patient were discussed. The multidisciplinary team concluded that the provisional diagnosis was a polypoid mass in D2, with suspicion of involvement of the ampulla of Vater. There was no proven malignancy based on the results of the pathology. However, the team concluded that the mass was still suspicious for malignancy. There was no possibility for endoscopic removal of the mass. Performing a pylorus preserving pancreaticoduodenectomy (PPPD) was decided as the best treatment option when she was able to withstand the stress of hospitalization and surgery. After the multidisciplinary team meeting, it was concluded that she might be 'frail' and at risk for adverse postoperative outcomes after a PPPD, based on several patient-related risk factors, such as age, COPD, cardiac problems, and functional dependence. Consequently, the patient was referred to the outpatient clinic (21 days after the diagnostic tests, see Figure 6.1) to discuss her treatment options with the surgeon. Seven days later, a preoperative multidisciplinary risk assessment in support of decision-making was performed as well. 


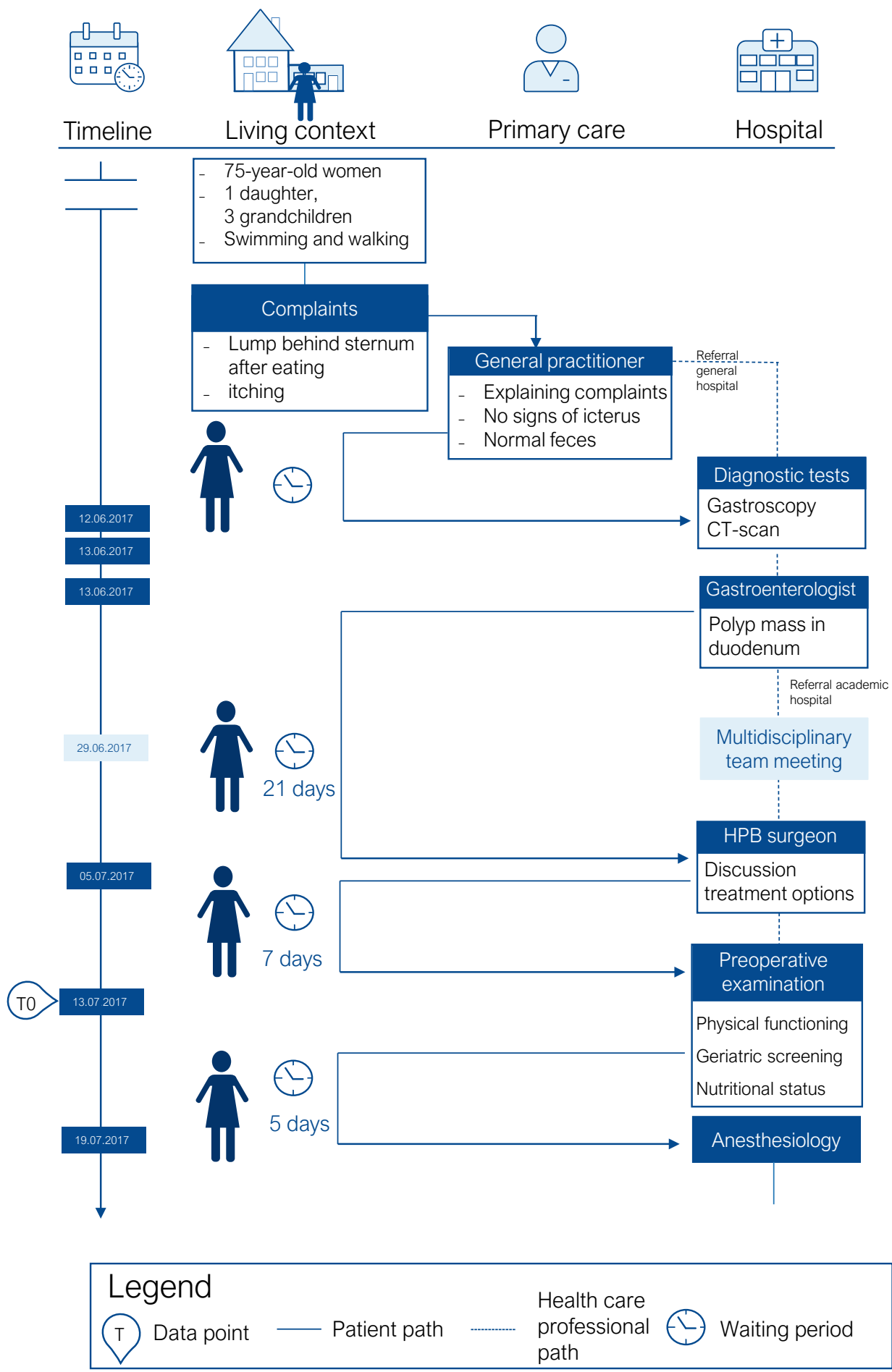




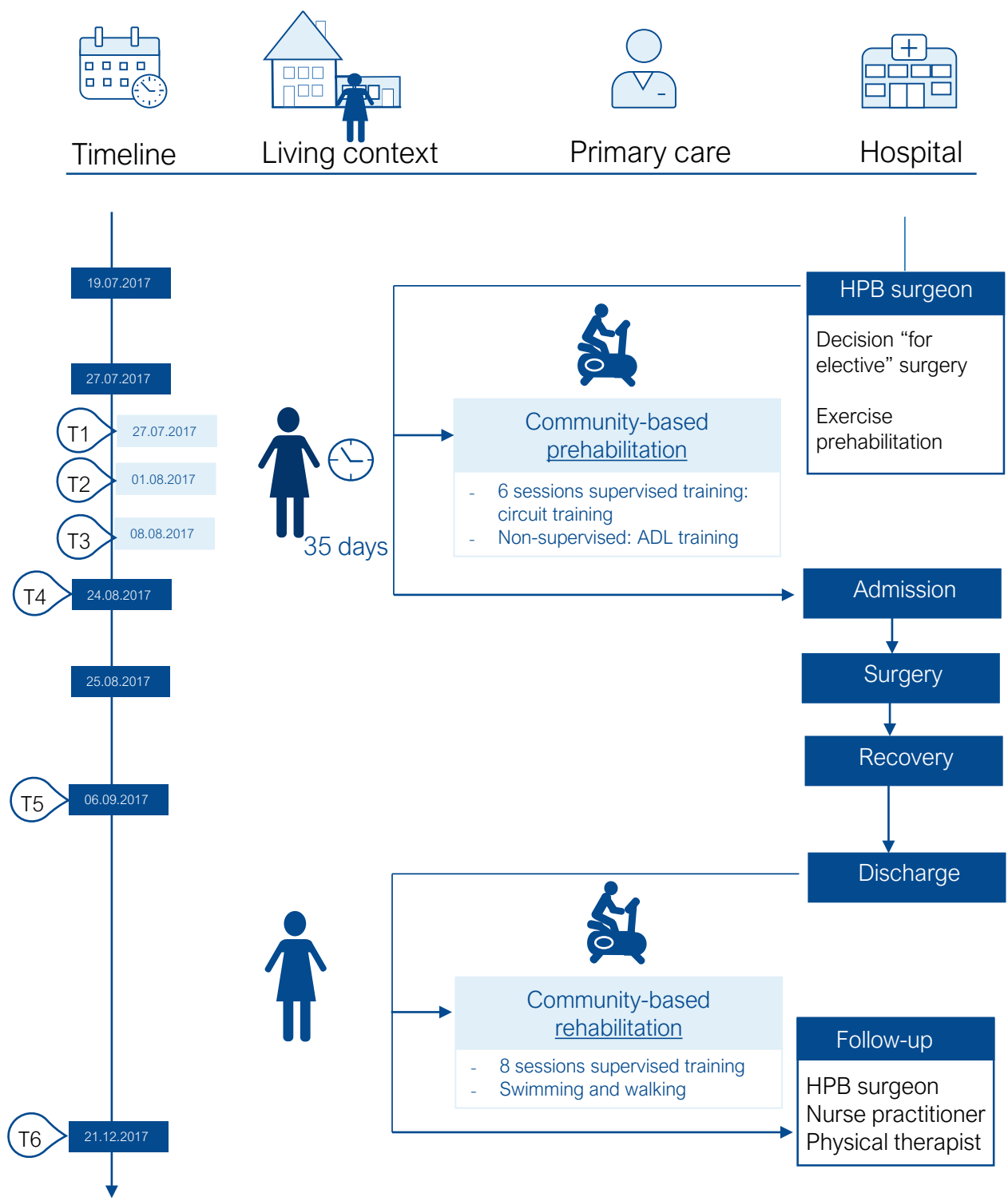

Figure 6.1. The patient journey listed on a timeline view ordered by start of the event. Abbreviations: $\mathrm{CT}=$ computed tomography; HPB=hepato-pancreato-biliary. 


\section{EXAMINATION STRATEGY}

\section{Rational for preoperative examination}

The HOAC II and the recently revised WHO framework of the ICF were used to structure and organize the patient history and clinical status, as well as to develop hypotheses prior to the preoperative examination and development of the plan of care (see Figure 6.2). The aim of the preoperative medical and functional examination (29 days after the diagnostic tests, see Figure 6.1) was to identify whether the patient was able to cope with the mental and physical stress of the pathology, hospitalization, and surgical stress response. The physiological response to stress can be described with the concepts of allostasis and allostatic load [13]. Allostasis is the process in which physiological systems adapt to excessive stressors to remain homeostasis. The cumulative effect of an allostatic state is allostatic load [13]. If additional load of unpredictable events (e.g., pathology, hospitalization, surgery) is superimposed on the already existing allostatic load (e.g., comorbidities), and the patient is not able to cope with this increased load, it may lead to allostatic overload, resulting in adverse health outcomes such as complications and a decline in physical functioning [13].

\section{Content of preoperative examination}

The aim was to assess the patient's medical condition and level of physical functioning to examine the patient's physiological reserve capacity (respiratory, cardiovascular, and physical functioning parameters, and nutritional and cognitive status) during the outpatient preoperative examination (see Figure 6.2, examination). The anesthesiologist assessed the general medical condition. The hospital physical therapist aimed to evaluate her level of physical functioning using performancebased tests to estimate her aerobic capacity (steep ramp test [SRT]), muscle strength (handgrip strength [HGS]), and functional mobility (timed up-and-go [TUG] test, two-minute walk test [2MWT], and the five times sit-to-stand [FTSTS] test). In addition, the physical therapist aimed to evaluate her perception of her functional capacity to perform activities of daily living using two questionnaires (veterans-specific activity questionnaire [VSAQ] and the Duke activity status index [DASI]). The nurse specialist evaluated the nutritional and cognitive status of the patient by performing a short-nutritional assessment, including anthropometric measurements (body height, body mass, circumference of the mid upper arm, triceps skinfold). An extensive description of the examination strategy of the physical therapist and clinical nurse specialist can be found in a Appendix 6.1. 


\section{Results of preoperative examination}

The anesthesiologist independently classified the patient with an American Society of Anesthesiologist (ASA) grade III, and concluded that she was able to perform activities on an activity level of 4 metabolic equivalents of task (METs). Results of the additional cardiac and respiratory examination are shown in Table 6.1. Results of the preoperative examination of physical functioning of the patient are presented in Figure 6.3 at time point T0. Nutritional assessment revealed an increase in body mass of $5 \mathrm{~kg}$ during the last six months. Moreover, no severe sarcopenic or cachexic state was observed by the nurse specialist. A comprehensive geriatric assessment was not recommended by the nurse specialist, despite a score $<14$ on the Geriatric 8 . The rationale here was that the patient's low Geriatric 8 score could be entirely attributed to her age, medications, and functional mobility.

\section{EVALUATION}

\section{Quantitative clinical impression II}

The examination supported the clinical impression that this patient had several risk factors that might be associated with adverse peri- and postoperative outcomes. Her advanced age (75 years), body mass index $\geq 25 \mathrm{~kg} / \mathrm{m}^{2}$, COPD (Gold II), cardiovascular disease (atrial fibrillation, left ventricular hypertrophy), American Society of Anesthesiologists (ASA) grade III, and her impaired functional mobility performance (see Figure 6.3, T0), are all independently associated with postoperative 30-day morbidity after pancreaticoduodenectomy [14-18]. An ASA grade $\geq 111$ is associated with severe (Clavien-Dindo grade IV or V) postoperative complications (odds ratio [OR] of 1.496; 95\% confidence interval [Cl] of 1.186 to $1.887 ; P=0.001$ ) (Schneider et al, 2012) and higher risk for postoperative pulmonary complications (OR of $3.12 ; 95 \% \mathrm{Cl}$ of 2.17 to 4.48 ) [19]. The results of the spirometry revealed moderate COPD grade II (Tiffeneau index $<70 \%$; $50 \%<\mathrm{FEV} 1<80 \%$ of predicted). Although respiratory distress is a predictor for postoperative complications, mild-to-moderate COPD is not independently and directly associated with an increased risk for postoperative pulmonary complications after abdominal surgery [20]. The risk factors for development of pulmonary complications depends not solely on the patient's respiratory comorbidity (COPD), but also on procedure-related factors (respiratory consequences of surgery and anesthesia [21] and context-related factors (early mobilization after surgery [22]. The dyspnea (shortness of breath and wheezing) noticed by the patient during ADL is typical for COPD patients and was noticed during the examination of her functional mobility performance. Consequently, this could explain the comprised walking distance on the 2MWT and the long duration at the TUG test. A TUG test score $>20 \mathrm{~s}$ is an independent predictor for 
postoperative delirium (hazard ratio of $4.8 ; 95 \% \mathrm{Cl}$ of 1.5 to $15.6 ; P=0.009$ ) [23], major postoperative complications (OR of $3.43 ; 95 \% \mathrm{Cl}$ of 1.13 to $10.36 ; P=0.03$ ) [24], and an increased length of stay of $>7$ days (OR of $4.21 ; 95 \% \mathrm{Cl}$ of 1.14 to $15.58 ; P=0.003$ ) [24] in elderly undergoing surgery. In addition, the aerobic capacity of the patient was indicated as 'poor' based on her inability to perform the modified SRT (10 W/10 s) and low MET values on the VSAQ (2.0 METs) and DASI (3.9 METs) questionnaires. A patient's aerobic capacity is associated with postoperative time to recovery of physical functioning, complications, length of stay, and mortality in major abdominal surgery $[25,26]$. Above findings support the limitations the patient experiences in ambulation, in walking long distances, and in performing household activities. These results correspond with the results of her scored self-reported physical activity level (VSAQ and DASI) and the estimated MET score of 4 METs by the anesthesiologist. Therefore, we expected that she was only able to perform activities with a light intensity (<4 METs).

Furthermore, approximately $30 \%$ of the patients $>70$ years develop a disability between the onset of their illness and their hospitalization or during their hospitalization resulting in loss in adequate performance of activities of daily living [1]. The development of a disability depends on several factors; pre-existing frailty, severity of the illness, and the hospital culture and infrastructure [1]. Physical inactivity and immobilization, due to augmented bed rest during hospitalization, is associated with impairments in transfers and walking due to loss of muscle mass [27]. Therefore, the patient's preoperative functional mobility should be sufficient to prevent her from entering a critical zone postoperatively, which might lead to a delayed recovery of physical functioning, permanent loss of physical functioning, morbidity, and/or mortality [5]. In conclusion, the patient's age, comorbidities, impaired functional mobility, and reduced aerobic capacity were considered risk factors for not being able to withstand the stress of hospitalization and surgery and, consequently, for having a higher risk for postoperative complications and an impaired recovery of physical functioning. 


\section{Plan of care}

The results of the medical and functional preoperative assessment and the identified risk factors were discussed directly after the examination with the patient by the anesthesiologist, physical therapist, and clinical nurse specialist. Subsequently, the surgeon discussed the risks and benefits of performing a PPPD (postoperative complications, mortality, potential malignancy, disease progression). Besides, she was educated about the importance of an adequate physical functioning level in relation to postoperative recovery of physical functioning and complications. Eventually, she opted for exercise prehabilitation to preoperatively improve her aerobic capacity, muscle strength, and functional mobility level, and thereby to reduce her risk for a delayed recovery or even permanent loss of physical functioning, morbidity, and mortality after surgery. There were no signs or symptoms for the need of nutritional or cognitive interventions. Although the patient did not like to perform exercise training (see quote), the patient and her surgeon agreed that she would benefit from participation in a prehabilitation program in order to reduce her perioperative risks and improve her recovery of physical functioning. Therefore, she decided to start preoperative exercise training at a community physical therapy practice in her neighborhood in order to preoperatively improve her level of physical functioning.

The patient

"The surgeon told me that if he would operate on me in my current health status, I would not leave the operation theatre alive. I needed more resilience and more reserves; he meant that I have to perform physical exercises. I thought, bah, I hate all that equipment." 


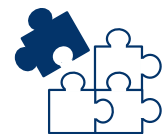

The HOAC II

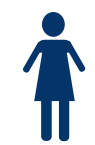

Patient data
ICF

Components

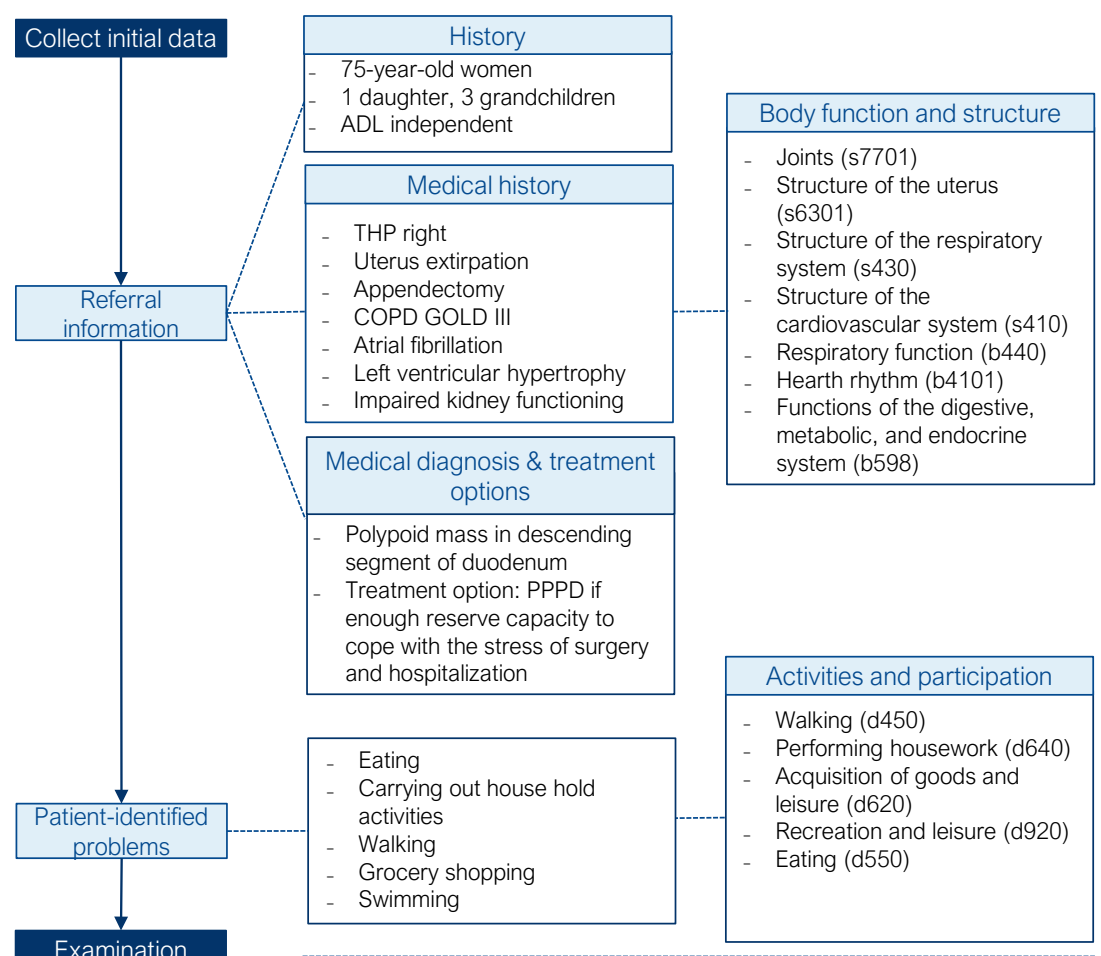

Examination

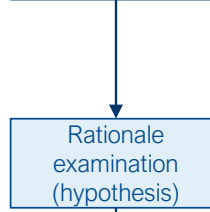

Personal factors

Functioning Participation

(hypothesis) 


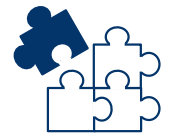

The HOAC II

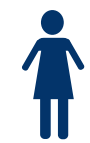

Patient data

\section{ICF}

\section{Components}

\begin{tabular}{|c|c|c|}
\hline \multirow{3}{*}{$\begin{array}{l}\text { Conduct } \\
\text { examination }\end{array}$} & Multidisciplinary screening & Body function and structure \\
\hline & \multirow{3}{*}{$\begin{array}{l}\text { Multidisciplinary screening to } \\
\text { asses the physiological reserves } \\
\text { - } \quad \text { Nutritional assessment } \\
\text { - } \quad \text { Geriatric assessment } \\
\text { - Physical functioning } \\
\text { - } \quad \text { Medical assessment }\end{array}$} & \multirow{3}{*}{$\begin{array}{ll}\text { - } & \text { Exercise tolerance (b455) } \\
\text { - } & \text { Muscle power function (b730) } \\
\text { - } & \text { Cardiovascular system (b410) } \\
\text { - } & \text { Respiratory function (b440) } \\
\text { - } & \text { Nutritional status } \\
- & \text { Cognitive status }\end{array}$} \\
\hline & & \\
\hline & & \\
\hline & \multirow{4}{*}{$\begin{array}{l}\text { I. Reduced physical activity level } \\
\text { related to comorbidities leading } \\
\text { to a poor cardiorespiratory } \\
\text { fitness level and muscle strength } \\
\text { II.Frailty, resulting from aging- } \\
\text { associated decline in } \\
\text { physiological systems leading to } \\
\text { disability in activities of daily life }\end{array}$} & Risk-factors \\
\hline \multirow{2}{*}{$\begin{array}{c}\text { Non-patient- } \\
\text { identified problems }\end{array}$} & & \multirow{3}{*}{$\begin{array}{ll}- & \text { Exercise tolerance (b455) } \\
\text { - } & \text { Muscle power function (b730) } \\
\text { - } & \text { Cardiovascular system (b410) } \\
- & \text { Respiratory function (b440) } \\
- & \text { Nutritional status } \\
- & \text { Cognitive status }\end{array}$} \\
\hline & & \\
\hline & & \\
\hline \multirow{2}{*}{$\begin{array}{l}\text { Generate } \\
\text { hypothesis }\end{array}$} & \multirow{2}{*}{\multicolumn{2}{|c|}{$\begin{array}{l}\text { Decreased physiological reserve (aerobic capacity level and muscle } \\
\text { strength) to adapt to the increase in allostatic load due to the pathology, } \\
\text { surgical stress, and hospitalization thereby at risk for adverse postoperative } \\
\text { outcome }\end{array}$}} \\
\hline & & \\
\hline \multirow[t]{2}{*}{$\begin{array}{l}\text { Identify rationale } \\
\text { for plan of care }\end{array}$} & \multicolumn{2}{|c|}{$\begin{array}{l}\text { Exercise prehabilitation to increase the physiological reserves } \\
\text { (cardiorespiratory and musculoskeletal fitness parameters) optimizing the } \\
\text { adaptation capacity to handle the surgical stress and hospitalization. }\end{array}$} \\
\hline & Patient level & Therapeutic level \\
\hline \multirow{2}{*}{ Establish goals } & $\begin{array}{l}\text { Increase ability to walk to the } \\
\text { shopping center. }\end{array}$ & \multirow{2}{*}{$\begin{array}{l}\text { - Improve aerobic capacity level } \\
\text { - Improve muscle strength } \\
\text { - } \quad \text { Improve functional mobility }\end{array}$} \\
\hline & 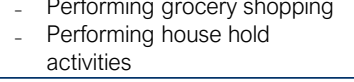 & \\
\hline
\end{tabular}

Figure 6.2. The step of the Hypothesis-Oriented-Algorithm for Clinicians II expanded with the International Classification of Functioning framework.

Abbreviations: $\mathrm{ADL}=$ activities of daily life; $\mathrm{COPD}=$ chronic obstructive pulmonary disease; $\mathrm{PPPD}=$ pylorus-preserving pancreaticoduodenectomy; THP=total hip replacement. 
Table 6.1. Results of the medical-, nutritional-, and geriatric assessment.

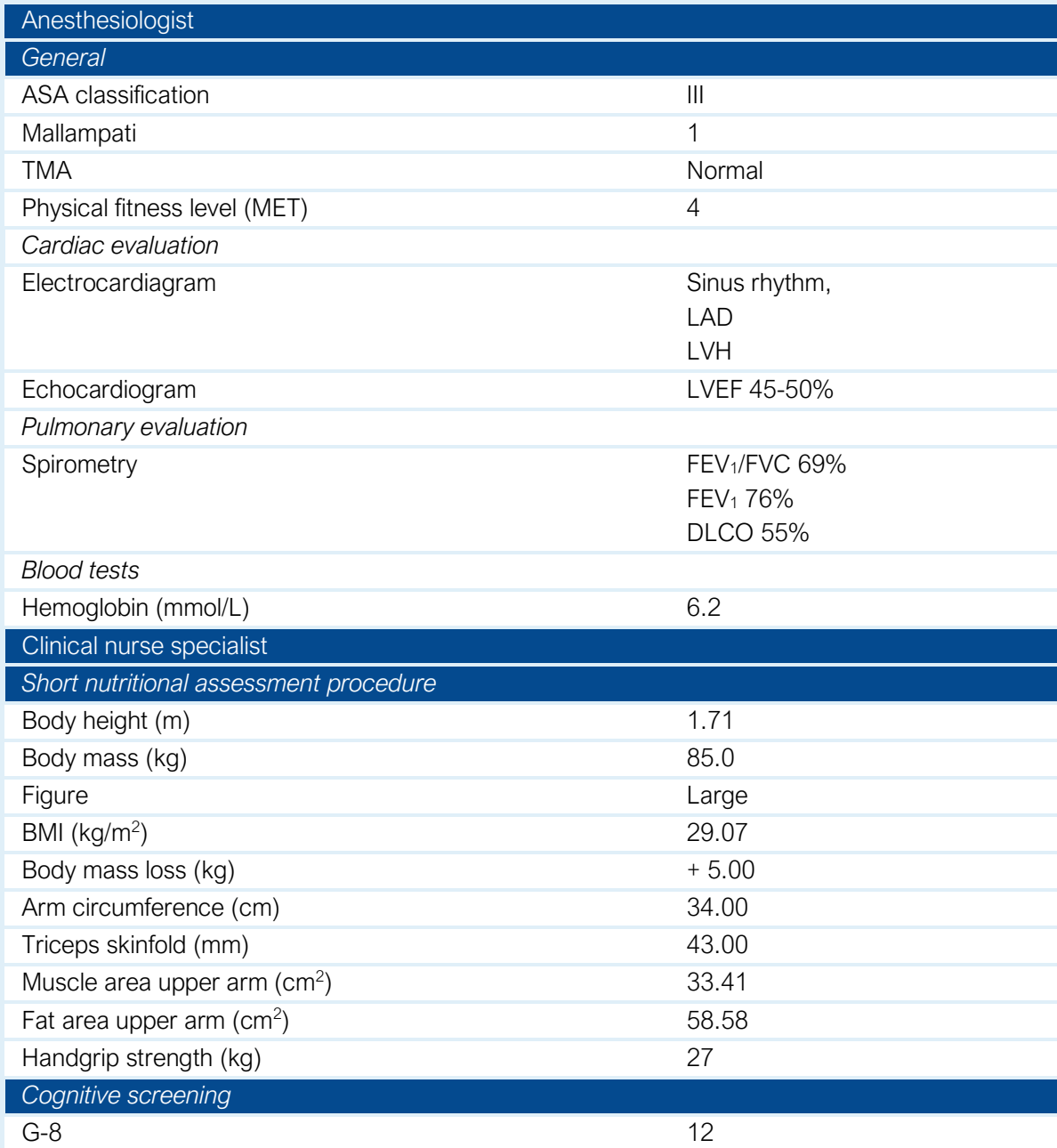

Abbreviations: ASA=American Society of Anesthesiologists; $\mathrm{DLCO}=$ diffusing capacity of carbon monoxide; FEV1=forced expiratory volume in one second; FVC=forced vital capacity; G8=Geriatric 8; $L A D=$ left axis deviation; $L V E F=$ left ventricular ejection fraction; $L V H=$ left ventricular hypertrophy; MET=metabolic equivalent of task; TMA=thyroid-mandible angle. 


\section{INTERVENTION}

Prehabilitation has shown to be beneficial in patients with a higher risk for postoperative complications by preoperatively improving their low aerobic capacity that might decrease their risk for postoperative complications $[28,29]$. A personalized prehabilitation program consisting of motivational interviewing, promotion of daily physical activity, and a supervised high-intensity training program in patients undergoing major elective abdominal surgery resulted in an increase in preoperative aerobic capacity and was found to be protective for the risk of postoperative complications (relative risk of $0.5 ; 95 \% \mathrm{Cl}$ of $0.3-0.8 ; P<0.001$ ) [30]. Besides the fact that the prehabilitation program should be personalized, training intensity should be high enough to protract substantial progression in a relatively short period before surgery, improving her physical functioning shifting her preoperative health status from a high-risk profile to a low-risk profile. The physical exercise training program should focus on functional task exercises required for the patient's ADL [31], and training progression should not only consist of increasing the number of repetitions and intensity, but on increasing complexity and variability as well. Frequent monitoring of progression by patients (self-monitoring) and their physical therapist using functional exercise tests is important to assess the patient's response to the prehabilitation program. The latter is an interventional procedure known as 'titration', aiming to use the maximal potential for improvement of a patient [32]. Target levels should be established at the start of the physical exercise training program and monitoring intervals should be short in the beginning to adequately record the expected asymptotic improvement in physical functioning following training sessions (law of diminishing returns) $[32,33]$. When test results are within reasonable predicted limits, the monitoring intervals may become longer. However, when the predicted and expected asymptotic curvature of training gains fails to appear (aspects of a non-responding patient), CONTENTissues [12] as prognosis and training parameters have to be reconsidered.

The patient participated in a partly supervised prehabilitation program at a nearby community physical therapy practice supplemented with functional task exercises she could perform at home. The four-week training program consisted of one-hour training sessions, which she attended twice per week. The aim of the preoperative physical exercise training program was to improve her aerobic capacity, muscle strength, and functional mobility using exercises in relation to ADL of relevance for her. The supervised training consisted of one-hour circuit training, including cycling, treadmill walking, resistance training, and functional task exercises, each lasting 15 minutes. For cycling, aerobic interval training on a cycle ergometer was performed, starting with three 4-minute intervals in the beginning of the training, which progressed to two 7- 
minute intervals in the last week. The initial work rate on the cycle ergometer was aimed to reach a Borg score 6-7 (0-10 Borg scale) for perceived exertion [34]. For the interval training at the treadmill, the average walking speed on the 2MWT was determined, as examined at the start of each new training week. Hence, she started with an average walking speed of $2.6 \mathrm{~km} / \mathrm{h}$ (2MWT distance of $86 \mathrm{~m}$ ). The interval training at the treadmill consisted of three 4-minute intervals at that walking speed. Intensity was verified with the 0-10 Borg scale (6-7) for perceived exertion. The resistance-training program included several exercises performed on weight training machines: leg press (quadriceps, hamstrings, gluteus, and calf muscles), leg extension (quadriceps), lat-pull down (latissimus dorsi and trapezius), chest press (pectoralis minor and major), and triceps pully (triceps). The intensity of the resistance training was set between 60 and $80 \%$ of the one repetition maximum (based on the Oddvar-Holten diagram), and the patient performed 3 sets of 8-12 repetitions, according to the Dutch guideline for resistance training in patients with chronic obstructive pulmonary disease [35]. Based on the 0-10 Borg scale for rating of perceived exertion, which should be 6 or 7 , resistance training intensity was progressed in order to maintain a sufficient training stimulus. Five functional task exercises were performed by the patient each week (sit-to-stand, weight carrying with bottles water or groceries, side-step, walking with walker, and stair climbing). These exercises were also the patient's homework exercises for the non-supervised part of the prehabilitation program. Every new training session at the beginning of the week her progression was objectively measured using the TUG test, FTSTS test, and the 2MWT. 

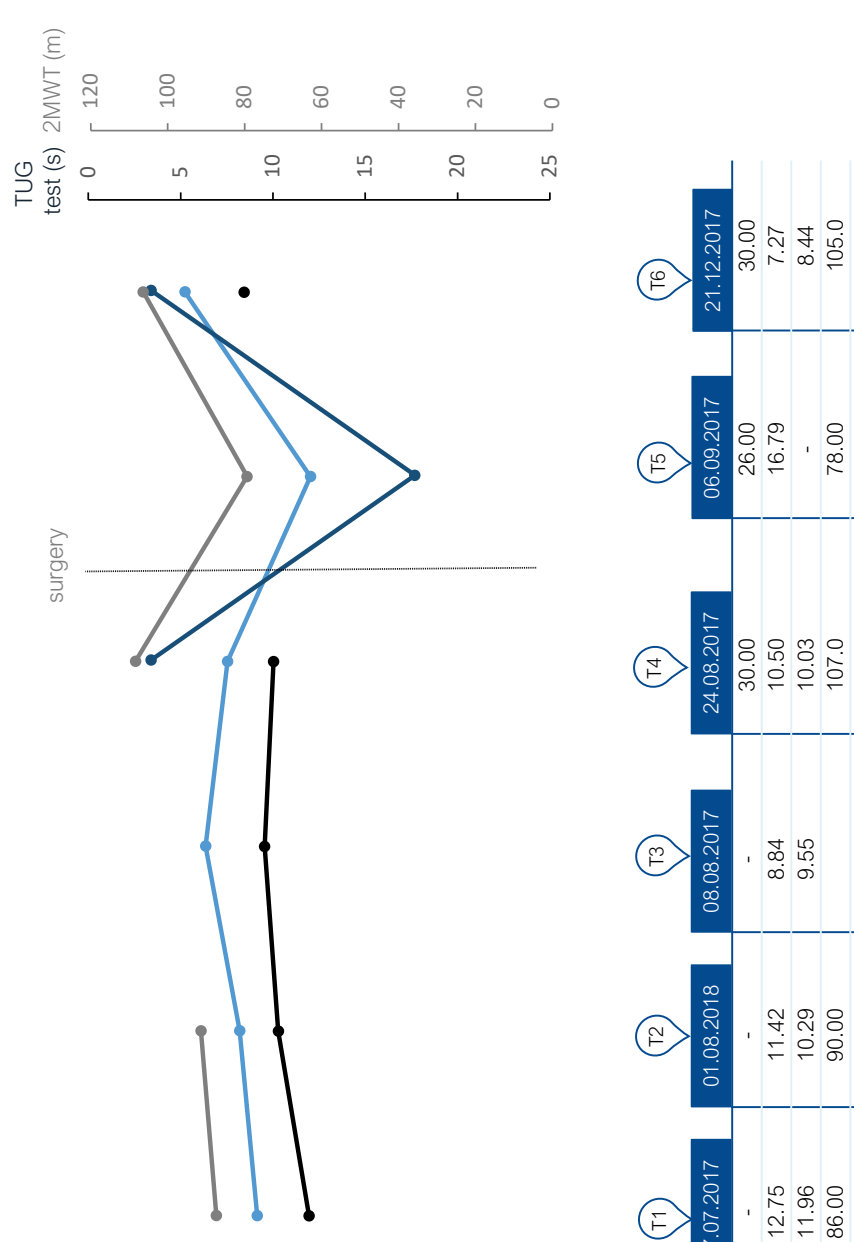

:

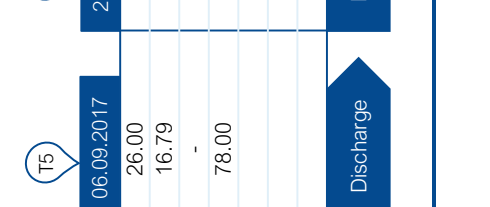

की
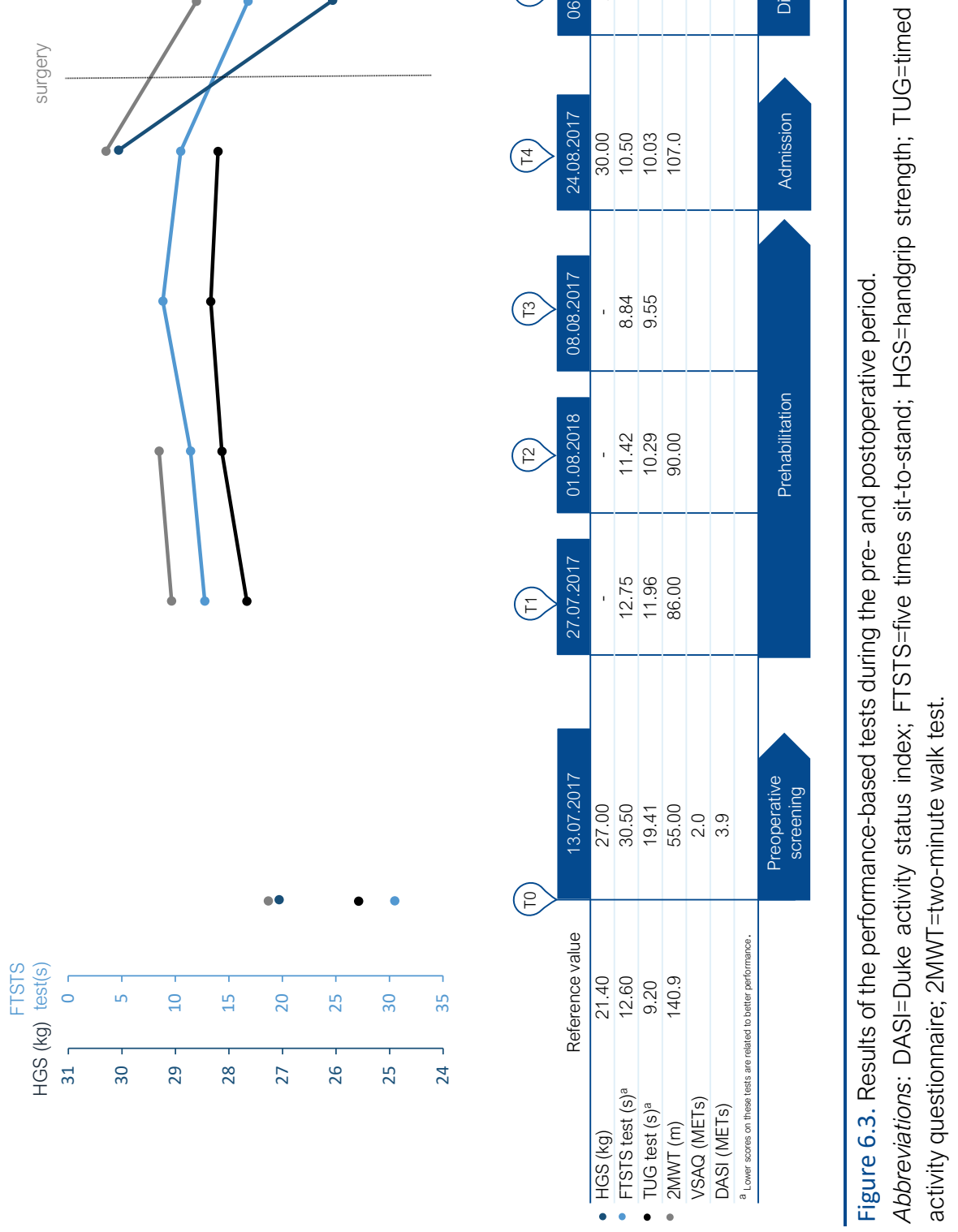


\section{OUTCOME}

\section{Prehabilitation}

The patient attended six preoperative exercise-training sessions. The adherence of the supervised training sessions was $75 \%$ (6/8 training sessions attended). The community physical therapist reported that the patient was highly motivated to participate in the personalized prehabilitation program. "For regular physical therapy in the past, she did not always complete her exercises; however, that was not the case this time, as she really wanted to undergo surgery, and she knew she had to improve her level of physical functioning preoperatively". She improved on all tests of functional mobility including TUG test performance (from 19.41 to $10.03 \mathrm{~s}$ ), FTSTS test performance (from 30.05 to $10.05 \mathrm{~s}$ ), and 2MWT performance (from 55.0 to $107.0 \mathrm{~m}$ ), thereby slightly showing an asymptotic training response curve (see Figure 6.3, T1-T4). In the last week of the prehabilitation program, she was not able to continue the two last supervised training sessions due to nausea. She continued her training the last week in her own living context. The perspective of the patient after three weeks of training was: "I noticed that I experienced less fatigue when performing ADL than before. Actually, I started to enjoy exercising, because I felt fitter. Consequently, I called the nurse specialist to ask when the surgery will be executed. It took me long enough to wait for surgery, while knowing it is going to happen anyway."

\section{Peroperative procedure and characteristics}

Four weeks after the initiation of the prehabilitation program, the patient was scheduled for her surgical procedure, a PPPD. A right subcostal incision was made to open the abdomen and the peritoneal cavity was searched for metastases. No peritoneal metastases were detected and intraoperative ultrasound showed no liver metastases. Peroperatively, a local excision of the duodenum polyp at the papillary of Vater was performed because of an à vue benign appearance, which was confirmed intra-operatively with frozen section investigation. For postoperative pain control, a wound catheter with continues infiltration of local anesthetic (bupivacaine $0.125 \%, 8$ $\mathrm{mL} / \mathrm{h}$ ) was placed in combination with patient-controlled morphine analgesia. Peroperative blood loss was $400 \mathrm{~mL}$, and the total duration of surgery was three hours and 34 minutes. The polyp was sent for examination to the department of pathology. A tubulo-villous adenoma with multifocal high-grade dysplasia was diagnosed. 


\section{Postoperative recovery and follow-up}

Postoperatively, the patient was sent deliberately to the intensive care unit because of her medical history. Physical therapy was initiated as of postoperative day (POD) 1, starting with optimizing respiratory gas-exchange using the active cycling breathing technique. She positioned herself in an upright position, under supervision of the physical therapist, to increase the diaphragm excursion and to decrease the work of breathing. At POD 2, she was discharged to the HPB ward and she started to participate in functional task training (e.g., practicing transfers, walking). The physical therapist visited the patient once or twice a day, depending on her performance and level of exhaustion. At POD 6, she achieved functional independency based on the modified lowa level of assistance scale without climbing stairs, as the latter was not relevant to her living status at home. At POD 7, functional mobility and muscle strength were re-assessed using the 2MWT, FTSTS test, and HGS (see Figure 6.3, T5). The patient showed a decrease in her functional

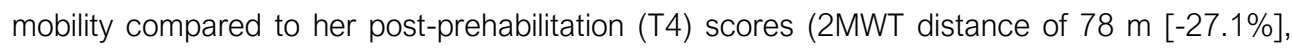
FTSTS test of $16.79 \mathrm{~s}$ [+59.9\%], and HGS of $26 \mathrm{~kg}$ [-13.3\%]). The hospitalization period was extended with 4 days because of a pre-existent staggering fluid balance; during these days, the patient remained functionally active and independent. The patient was discharged home at POD 12. At home, she resumed the physical exercise training program at the community physical therapy practice twice a week for four weeks. The aim of this supervised rehabilitation program was to regain physical functioning to the patient's level experienced and measured before diagnosis, focusing on aerobic and resistance training. The same training principles of the prehabilitation program were also applied for the rehabilitation program. After the supervised rehabilitation program, she picked up swimming again (once or twice a week), accompanied with more walking activities. These activities were not monitored by the community physical therapist. Three months after surgery the practical performance based-tests were performed by the hospital physical therapist to re-assess her level of physical functioning and self-reported perceived level of physical functioning. She performed at the same level as preoperatively measured after the prehabilitation program (see Figure 6.3, T6).

\section{DISCUSSION}

This case report illustrates the identification of risks and therapeutic opportunities for prehabilitation with a patient with several risk factors for a complicated postoperative recovery. Using concepts as the HOAC II, ICF, and the CONTENT scale on the one hand, and evidence like the strong relation between preoperative physical functioning and postoperative outcomes $[25,26]$, the importance of preoperative risk-stratification [26], as well as the beneficial effects of 
prehabilitation $[28,30]$ on the other hand, the patient and her (in)formal caregivers as a team were able to improve her preoperative physical functioning in a real-life clinical context. Upon this prehabilitation trajectory, no postoperative complications and a fairly rapid recovery of physical functioning (6 days) occurred, followed by a continued improvement of her physical functioning after hospital discharge. The peroperative risk for morbidity and mortality during the surgical procedure were, in our opinion, only marginally determined by the change from a PPPD to a local resection of the ampulla of Vater, given that the preparation and anesthesiology were completed for a PPPD. Postoperatively, the risks for a delayed recovery or permanent loss of physical functioning, morbidity, and mortality are certainly slightly reduced after this procedure compared to a PPPD. Given the preparation with abdominal mobilization and the risk of leakage of pancreatic fluids, this procedure should still be classified as an abdominal surgical procedure with high risk for delayed or permanent loss of physical functioning, morbidity, and eventually mortality.

Identification of a patient's preoperative risk profile often relies on a classification system with conventional factors, such as age, ASA score, and comorbid conditions [14-18]. However, adding variables of aerobic capacity, muscle strength, and functional mobility to conventional prediction models significantly improves the predictive value of postoperative outcomes [36-38]. In our case, the modified SRT to estimate aerobic capacity was not possible due to dyspnea complaints. However, the additional practical performance-based tests seemed to be able to provide insight in her physiological reserve capacity. Although literature addressing practical performance-based tests of physical functioning in relation to postoperative outcomes in patients undergoing a pancreaticoduodenectomy is limited, measurements of functional mobility in terms of ADL performance is important and related to the postoperative course in patients undergoing major abdominal surgery $(25,36,39,40]$. Frequently performing these tests during the prehabilitation trajectory by the patient, their (in)formal caregivers, as well as the physical therapist should be encouraged to monitor progression and ensure optimal exercise dosage. Additionally, repeated monitoring progression might have beneficial motivational effects in responders; however, training should be adapted in case of non-responders because it might exert discouraging effects.

As presented in this case report, a short-term preoperative physical exercise training program is feasible and effective in a high-risk frail patient opting for major abdominal surgery. The given exercise training in this case report can be considered therapeutically valid, since it contained seven out of nine items on the CONTENT scale. Previous reports about prehabilitation programs in patients undergoing major abdominal surgery show heterogeneous designs in terms of patient 
selection, modalities (aerobic training, resistance training, inspiratory muscle training, or a combination), intensity (low to high training intensity), context (home-, community-, and/or hospital-based), and duration of training [41]. The use of resistance training in the training program focusing on individual muscle groups is debatable, since functional task training involves training of these muscle groups as well. Functional task training has been reported to be more effective in the improvement of ADL in elderly compared to resistance training [31] and feasible in highly compromised elderly [42]. The patient improved in her physical functioning in which the greatest improvement was seen in the first two weeks after the preoperative assessment. This could be due to a suboptimal performance during the assessment in the hospital, or because the patient became more physically active after her preoperative examination.

In order to preserve and to optimally make use of the effects of prehabilitation, a physically activating culture and infrastructure in both its context and processes in the hospital is a prerequisite to regain and preserve independent physical functioning in $A D L$ as soon as possible after surgery [5]. Sedentary behavior is however provoked, as the dominant hospital recovery strategy is still bed-centered care. A case report like this has the potential to address these issues and enables to discuss 'habits' in care processes and procedures. Improvement of the hospital clinical context at hand here, may potentiate the recovery of physical functioning of patients during hospitalization in the future. Furthermore, by evaluating single cases over time with scientific evidence, a multidisciplinary learning community could be developed, improving clinical practice and facilitating and evaluating health care innovations ensuring research, quality and value-adding improvements in real-life clinical practice.

In the light of a gradual transition of stratified care to personalized health care, studying the perioperative trajectory and results of - in this case - an embedded prehabilitation phase in a single patient should be seen as a first step. This case report grands the opportunity to carefully and explicitly learn from practice and intertwined research. Moreover, in-depth analysis of a single case provides clinicians and researchers with detailed information on how all diagnostic and therapeutic interventions are executed and how their social, physical, and psychological responses unfold in real-life practice [43]. With the use of the HOAC II for clinical reasoning, the ontology of the ICF, the CARE guidelines, and the CONTENT scale, an effort has been made to increase the internal validity and provide transparency and options for replicating the study and critical evaluation of the cause-effect considerations. Nevertheless, case studies are known for their scarce external validity [44] and should therefore be replicated in order to be able to draw conclusions for the population addressed in the study. By combining multiple single case studies, 


\section{Chapter 6}

the variability of patient responses to a certain intervention can be explored in its clinical or reallife variability.

This case report demonstrates how preoperative risk assessment can support clinical decisionmaking in coalition with a high-risk patient opting for major abdominal surgery and shows the feasibility and effects of preoperative physical exercise training in this high-risk patient. Furthermore, this case report illustrates how perioperative care can be improved by discussing evidence and reflecting on 'standard' perioperative care interventions in a single high-risk patient.

\section{ACKNOWLEDGEMENTS}

We would like to thank Maud Maassen (community physical therapist, Fysio GMS, Heerlen, the Netherlands) and Lieke Corpelijn (clinical nurse specialist, Maastricht University Medical Center+) for their contribution to the study. 
1. Covinsky KE, Pierluissi E, Johnston CB. Hospitalization-associated disability: "She was probably able to ambulate, but I'm not sure". JAMA. 2011;306:1782-93.

2. Lawrence VA, Hazuda HP, Cornell JE, Pederson T, Bradshaw PT, Mulrow CD, et al. Functional independence after major abdominal surgery in the elderly. J Am Coll Surg. 2004;199:762-72.

3. Palleschi L, Fimognari FL, Pierantozzi A, Salani B, Marsilii A, Zuccaro SM, et al.Acute functional decline before hospitalization in older patients. Geriatr Gerontol Int. 2014;14:769-77.

4. Dronkers J, Witteman B, van Meeteren N. Surgery and functional mobility: doing the right thing at the right time. Tech Coloproctol. 2016;20:339-341.

5. Hulzebos EH, van Meeteren NL. Making the elderly fit for surgery. $\mathrm{Br} \mathrm{J}$ Surg.2016;103:463.

6. West MA, Wischmeyer PE, Grocott MPW. Prehabilitation and nutritional support to improve perioperative outcomes. Curr Anesthesiol Rep. 2017;7:340-49.

7. Hood L. Systems biology and P4 medicine: past, present, and future. Rambam Maimonides Med J. 2013;4:e0012.

8. Bongers BC, Punt IM, van Meeteren NL. On "Prehabilitation: The emperor's new clothes or a new a rena for physical therapists?" Lundberg M, Archer KR, Larsson C, Rydwik E. Phys Ther. 2018;12:127-130. Phys Ther. 2019 Jul 1;99:953-54.

9. Rothstein JM, Echternach JL, Riddle DL. The Hypothesis-Oriented Algorithm for Clinicians II (HOAC II): a guide for patient management. Phys Ther. 2003;83:455-70.

10. Heerkens YF, de Weerd M, Huber M, de Brouwer CPM, van der Veen S, Perenboom RJM, et al. Reconsideration ICF scheme. Disabil Rehabil. 2018;40:12122.

11. Gagnier JJ, Kienle G, Altman DG, Moher D, Sox H, Riley D; CARE Group. The CARE Guidelines: Consensus-based Clinical Case Reporting Guideline Development. Glob Adv Health Med. 2013;2:38-43.
12. Hoogeboom TJ, Oosting E, Vriezekolk JE, Veenhof C, Siemonsma PC, de Bie RA, et al. Therapeutic validity and effectiveness of preoperative exercise on functional recovery after joint replacement: a systematic review and meta-analysis. PLoS One. 2012;7:e38031.

13. McEwen BS, Wingfield JC. What is in a name? Integrating homeostasis, allostasis and stress. Horm Behav. 2010;57:105-11.

14. DeOliveira ML, Winter JM, Schafer M, Cunningham SC, Cameron JL, Yeo CJ, et al. Assessment of complications after pancreatic surgery: A novel grading system applied to 633 patients undergoing pancreaticoduodenectomy. Ann Surg. 2006;244:931-7.

15. Greenblatt DY, Kelly KJ, Rajamanickam V, Wan Y, Hanson T, Rettammel R, et al. Preoperative factors predict perioperative morbidity and mortality after pancreaticoduodenectomy. Ann Surg Oncol. 2011;18:2126-35.

16. Schneider $\mathrm{EB}$, Hyder $\mathrm{O}$, Wolfgang $\mathrm{CL}$, Hirose K, Choti MA, Makary MA, et al. Patient readmission and mortality after surgery for hepato-pancreato-biliary malignancies. J Am Coll Surg. 2012;215:607-15.

17. Venkat R, Puhan MA, Schulick RD, Cameron JL, Eckhauser FE, Choti MA, et al. Predicting the risk of perioperative mortality in patients undergoing pancreaticoduodenectomy: a novel scoring system. Arch Surg. 2011;146:1277-84.

18. Wiltberger G, Muhl B, Benzing C, Atanasov G, Hau HM, Horn M, et al. Preoperative risk stratification for major complications following pancreaticoduodenectomy: Identification of high-risk patients. Int J Surg. 2016;31:33-9.

19. Smetana GW, Lawrence VA, Cornell JE; American College of Physicians.

Preoperative pulmonary risk stratification for noncardiothoracic surgery: systematic review for the American College of Physicians. Ann Intern Med. 2006;144:581-95. 
20. Wagner D, Büttner S, Kim Y, Gani F, Xu L, Margonis GA, et al. Clinical and morphometric parameters of frailty for prediction of mortality following hepatopancreaticobiliary surgery in the elderly. Br J Surg.2016;103:e83-92.

21. Licker M, Schweizer A, Ellenberger C, Tschopp JM, Diaper J, Clergue F. Perioperative medical management of patients with COPD. Int J Chron Obstruct Pulmon Dis. 2007;2:493-515.

22. Haines KJ, Skinner EH, Berney S; Austin Health POST Study Investigators.

Association of postoperative pulmonary complications with delayed mobilisation following major abdominal surgery: an observational cohort study. Physiotherapy. 2013;99:119-25.

23. Brouquet $\mathrm{A}$, Cudennec $\mathrm{T}$, Benoist $\mathrm{S}$, Moulias S, Beauchet A, Penna C, et al. Impaired mobility, ASA status and administration of tramadol are risk factors for postoperative delirium in patients aged 75 years or more after major abdominal surgery. Ann Surg. 2010;251:759-65.

24. Huisman MG, van Leeuwen BL, Ugolini G, Montroni I, Spiliotis J, Stabilini C, et al. "Timed Up \& Go": a screening tool for predicting 30-day morbidity in oncogeriatric surgical patients? A multicenter cohort study. PLoS One. 2014;9:e86863.

25. Heldens AFJM, Bongers BC, Lenssen AF, Stassen LPS, Buhre WF, van Meeteren NLU. The association between performance parameters of physical fitness and postoperative outcomes in patients undergoing colorectal surgery: An evaluation of care data. Eur J Surg Oncol. 2017 ;43:2084-92.

26. Moran J, Wilson F, Guinan E, McCormick P, Hussey J, Moriarty J. Role of cardiopulmonary exercise testing as a riskassessment method in patients undergoing intra-abdominal surgery: a systematic review. Br J Anaesth. 2016;116:177-91.

27. Ikezoe T, Nakamura M, Shima H, Asakawa $\mathrm{Y}$, Ichihashi N. Association between walking ability and trunk and lower-limb muscle atrophy in institutionalized elderly women: a longitudinal pilot study. J Physiol Anthropol. 2015;34:31.
28. Moran J, Guinan E, McCormick P, Larkin J, Mockler D, Hussey J, et al. The ability of prehabilitation to influence postoperative outcome after intra-abdominal operation: A systematic review and meta-analysis. Surgery. 2016;160:1189-1201.

29. Thomas G, Tahir MR, Bongers BC, Kallen VL, Slooter GD, van Meeteren NL. Prehabilitation before major intraabdominal cancer surgery: a systematic review of randomised controlled trials. Eur J Anaesthesiol. 2019;36:933-45

30. Barberan-Garcia A, Ubré M, Roca J, Lacy AM, Burgos F, Risco R, et al. Personalised prehabilitation in high-risk patients undergoing elective major abdominal surgery: a randomized blinded controlled trial. Ann Surg. 2018;267;50-6.

31. de Vreede PL, Samson MM, van Meeteren NL, Duursma SA, Verhaar HJ. Functionaltask exercise versus resistance strength exercise to improve daily function in older women: a randomized, controlled trial. J Am Geriatr Soc. 2005;53:2-10.

32. Glasziou P, Irwig L, Mant D. Monitoring in chronic disease: a rational approach. BMJ. 2005;330:644-8.

33. Hoffman J. Physiological aspects of sport training and performance. Champaign: Human Kinetics. 2014:93-102.

34. Horowitz MB, Littenberg B, Mahler DA. Dyspnea ratings for prescribing exercise intensity in patients with COPD. Chest. 1996;109:1169-75.

35. Gosselink R, Langer D, Burtin C, Probst V, Hendriks $\mathrm{H}$, van der Schans $\mathrm{C}$, et al. KNGF-richtlijn chronisch obstructieve longziekten. Nederlands Tijdschrift voor Fysiotherapie. 2008;118: 1-60.

36. Dronkers JJ, Chorus AM, van Meeteren NL, Hopman-Rock M. The association of pre-operative physical fitness and physical activity with outcome after scheduled major abdominal surgery. Anaesthesia. 2013;68:67-73.

37. Ausania F, Snowden CP, Prentis JM, Holmes LR, Jaques BC, White SA, et al. Effects of low cardiopulmonary reserve on pancreatic leak following pancreaticoduodenectomy. Br J Surg. 2012 ;99:1290-4. 
38. Wijeysundera DN, Pearse RM, Shulman MA, Abbott TEF, Torres E, Ambosta A, et al. Assessment of functional capacity before major non-cardiac surgery: an international, prospective cohort study. Lancet. 2018;391;::2631-40.

39. Hayashi K, Yokoyama Y, Nakajima $\mathrm{H}$, Nagino M, Inoue T, Nagaya M, et al. Preoperative 6-minute walk distance accurately predicts postoperative complications after operations for hepatopancreato-biliary cancer. Surgery. 2017; 161:525-32.

40. Van Beijsterveld CA, Bongers BC, Den Dulk M, Van Kuijk SMJ, Dejong CH, Van Meeteren NL. The association between preoperative physical functioning and short-term postoperative outcomes: a cohort study of patients undergoing elective hepatic resection. HPB (Oxford). 2019;21:1362-70.

41. Berkel AEM, Bongers BC, van Kamp MS, Kotte $\mathrm{H}$, Weltevreden $\mathrm{P}$, de Jongh FHC, et al. The effects of prehabilitation versus usual care to reduce postoperative complications in high-risk patients with colorectal cancer or dysplasia scheduled for elective colorectal resection: study protocol of a randomized controlled trial. BMC Gastroenterol. 2018;18:29.
42. Siemonsma PC, Blom JW, Hofstetter $\mathrm{H}$, van Hespen ATH, Gussekloo J, Drewes YM, et al. The effectiveness of functional task exercise and physical therapy as prevention of functional decline in community dwelling older people with complex health problems. BMC Geriatr. 2018;18:164.

43. Harris SR. Revisiting the Case for Case Reports. Phys Ther. 2019 ;99:1122-5.

44. Lillie EO, Patay B, Diamant J, Issell B, Topol EJ, Schork NJ. The n-of-1 clinical trial: the ultimate strategy for individualizing medicine? Per Med. 2011;8:161-73. 
Chapter 6

\section{APPENDIX}

Appendix 6.1. Examination physical therapist and clinical nurse specialist

\section{Examination physical therapist}

Self-reported physical activity level.

The patient's perception of her functional capacity in performing activities of daily living was measured using the veterans-specific activity questionnaire (VSAQ) and the Duke activity status index (DASI). Both questionnaires consist of a list of activities linked to a particular metabolic equivalent of task (MET) score [1-3]. The VSAQ has a good intra- (ICC of $0.88 ; 95 \% \mathrm{Cl}$ of 0.76 to 0.94 ) and inter-rater reliability (ICC of $0.90 ; 95 \% \mathrm{Cl}$ of 0.80 to 0.95$)$ [4]. Both VSAQ (Vo 2peak, ICC of 0.57 , standard errors of estimate $7.63 \mathrm{~mL} / \mathrm{kg} / \mathrm{min}, P<0.001)$ and the DASI (Vozpeak, spearman correlation coefficient of 0.81 ) correlate moderate to good with aerobic capacity $[1,5]$.

\section{Functional mobility}

Functional mobility was measured using the timed up-and-go (TUG) test (in s), two-minute walk test (2MWT, in m), and the five times sit-to-stand (FTSTS) test (in s). The TUG test measures the ability to rise from an arm chair (43 to $47 \mathrm{~cm}$ in height), walk a short distance $(3 \mathrm{~m}$ ), return to the chair, and sit down again, all as quickly as possible [6]. The TUG test is associated with time to recovery of physical functioning (odds ratio [OR] of $1.274 ; 95 \% \mathrm{Cl}$ of 0.975 to $1.664 ; P=0.008$ ) scored on the modified lowa level of assistance scale (mILAS) in patients undergoing colorectal surgery [7]. During the 2MWT, patients have to walk as far as they can in two minutes over a length of 15 meters with their customary walking aid, if applicable. The test has shown to have a good test-retest reliability (intra-class correlation coefficient (ICC) of $0.82 ; 95 \% \mathrm{Cl}$ of 0.76 to 0.87 ) in healthy adults between 18 to 85 years [8]. The FTSTS test is a performance-based functional test evaluating the combination of functional mobility, balance, and lower leg muscle strength $[9,10]$. Patients are asked to stand up and sit down from a chair (43 to $47 \mathrm{~cm}$ in height) as quickly as possible for five times with their arms folded across their chest. The FTSTS test is easy to perform in clinical practice and is reliable in community-dwelling older people (ICC of 0.89; 95\% $\mathrm{Cl}$ of 0.79 to 0.95$)$ [10].

\section{Muscle strength}

Evaluation of handgrip strength (HGS, in $\mathrm{kg}$ ) of the dominant hand was performed to provide insight in general muscle strength using a hand-held dynamometer (JAMARß Hydraulic Hand Dynamometers, JAMAR, Patterson, Medical Holdings, Inc., Ilinois, USA). Impaired preoperative 
HGS is related with increased postoperative morbidity, mortality, and prolonged hospital stay following surgery [11-13].

\section{Aerobic capacity}

To estimate aerobic capacity a steep ramp test (SRT) was performed. The SRT is a short-time maximal exercise test performed on a cycle ergometer (Lode Corival Rehab, Lode BV, Groningen, the Netherlands), in which the attained peak work rate (WR peak, in W) is the primary outcome parameter. A modified incremental ramp protocol (10 W/10 s instead of the original 25 W/10 s) was used, as the duration of the test should be long enough to ensure sufficient involvement of the aerobic energy system. De Backer et al. found a strong correlation between aerobic capacity (Vo $\mathrm{V}_{\text {peak, }}$ in $\mathrm{mL} / \mathrm{min}$ ) attained during a cardiopulmonary exercise test and the achieved $W_{\text {peak }}$ at the original SRT protocol $(r=0.82 ; P<0.001)$ [14]. In pancreatic cancer surgery, patients with a low aerobic capacity (oxygen uptake at the ventilatory anaerobic threshold $(<10 \mathrm{~mL} / \mathrm{kg} / \mathrm{min}$ ) were found to be at an increased risk of postoperative complications (pancreatic fistula $(P=0.028)$ and major intra-abdominal abscesses $(P=0.042)$, as well as an increased hospital length of stay (hazard ratio of $1.74 ; 95 \% \mathrm{Cl}$ of 1.14 to $2.64 ; P=0.010$ ) [15].

\section{Examination clinical nurse specialist}

The clinical nurse specialist used two time-efficient screening methodologies to identify whether the patient was vulnerable and whether a referral for a comprehensive geriatric assessment and full nutritional assessment was necessary.

\section{Geriatric screening}

The G-8 screening tool consists of eight questions concerning age (years), nutritional status, loss of body mass (in $\mathrm{kg}$ ), body mass index (BMI, in $\mathrm{kg} / \mathrm{m}^{2}$ ), motor skills, psychological status, number of medications, and self-perception of health.16-18 The G-8 is considered to be the most sensitive (ranges from 65\%-92\%) screening tool for frailty in older cancer patients [19].

\section{$\underline{\text { Nutritional status }}$}

The short nutritional assessment procedure (SNAP) is able to identify and monitor patients with a low fat free mass index and low muscle strength [20]. The SNAP consists of the following anthropometric measurements: body height, body mass, circumference of the mid upper arm, triceps skinfold, and HGS. Based on these measurements, BMI $\left(\mathrm{kg} / \mathrm{m}^{2}\right)$, muscle area upper arm (cm2), and fat area of the upper arm (cm2) can be calculated. 


\section{References Appendix}

1. Hlatky MA, Boineau RE, Higginbotham MB, Lee KL, Mark DB, Califf RM, et al. A brief self-administered questionnaire to determine functional capacity (the Duke Activity Status Index). Am J Cardiol. 1989;64:651-4.

2. Myers J, Bader D, Madhavan R, Froelicher $\checkmark$. Validation of a specific activity questionnaire to estimate exercise tolerance in patients referred for exercise testing. Am Heart J. 2001;142:1041-6.

3. Myers J, Do D, Herbert W, Ribisl P, Froelicher VF. A nomogram to predict exercise capacity from a specific activity questionnaire and clinical data. Am J Cardiol. 1994;73:591-6.

4. de Carvalho Bastone A, de Souza Moreira B, Teixeira CP, Dias JM, et al. Is the veterans specific activity questionnaire valid to assess older adults aerobic fitness? J Geriatr Phys Ther. 2016;39:117-24.

5. da Silva SC, Monteiro WD, Cunha FA, Myers J, Farinatti PT. Determination of best criteria to determine final and initial speeds within ramp exercise testing protocols. Pulm Med. 2012;542402.

6. Podsiadlo D, Richardson S. The timed "Up \& Go": a test of basic functional mobility for frail elderly persons. J Am Geriatr Soc. 1991;39:142-8.

7. Heldens AFJM, Bongers BC, Lenssen AF, Stassen LPS, Buhre WF, van Meeteren NLU. The association between performance parameters of physical fitness and postoperative outcomes in patients undergoing colorectal surgery: An evaluation of care data. Eur J Surg Oncol. 2017;43:2084-92.

8. Bohannon RW, Wang YC, Gershon RC. Two-minute walk test performance by adults 18 to 85 years: normative values, reliability, and responsiveness. Arch Phys Med Rehabil. 2015;96:472-7.

9. Lord SR, Murray SM, Chapman K, Munro $B$, Tiedemann A. Sit-to-stand performance depends on sensation, speed, balance, and psychological status in addition to strength in older people. J Gerontol A Biol Sci Med Sci. 2002;57:M539-43.
10. Tiedemann A, Shimada H, Sherrington $C$, Murray S, Lord S. The comparative ability of eight functional mobility tests for predicting falls in community-dwelling older people. Age Ageing. 2008;37:430-5.

11. Mahalakshmi VN, Ananthakrishnan N, Kate V, Sahai A, Trakroo M. Handgrip strength and endurance as a predictor of postoperative morbidity in surgical patients: can it serve as a simple bedside test? Int Surg. 2004;89:115-21.

12. Chen $\mathrm{CH}$, Ho-Chang, Huang $\mathrm{YZ}$, Hung TT. Hand-grip strength is a simple and effective outcome predictor in esophageal cancer following esophagectomy with reconstruction: a prospective study. J Cardiothorac Surg. 2011;6:98.

13. Webb AR, Newman LA, Taylor M, Keogh JB. Hand grip dynamometry as a predictor of postoperative complications reappraisal using age standardized grip strengths. JPEN J Parenter Enteral Nutr. 1989;13:303.

14. De Backer IC, Schep G, Hoogeveen A, Vreugdenhil G, Kester AD, van Breda E. Exercise testing and training in a cancer rehabilitation program: the advantage of the steep ramp test. Arch Phys Med Rehabil. 2007;88:610-6.

15. Chandrabalan VV, McMillan DC, Carter R, Kinsella J, McKay CJ, Carter CR, et al. Preoperative cardiopulmonary exercise testing predicts adverse post-operative events and non-progression to adjuvant therapy after major pancreatic surgery. HPB (Oxford). 2013;15:899-907.

16. Bellera CA, Rainfray M, Mathoulin-Pélissier S, Mertens C, Delva F, Fonck M, et al. Screening older cancer patients: first evaluation of the G-8 geriatric screening tool. Ann Oncol. 2012;23:2166-72.

17. Soubeyran P, Bellera C, Goyard J, Heitz D, Curé $\mathrm{H}$, Rousselot $\mathrm{H}$ et al. oulin-Pélissier Screening for vulnerability in older cancer patients: the ONCODAGE Prospective Multicenter Cohort Study. PLoS One. 2014;9:e115060. 
18. Kenis C, Decoster L, Van Puyvelde K, De Grève J, Conings G, Milisen K, et al. Performance of two geriatric screening tools in older patients with cancer. J Clin Oncol. 2014;32:19-26.

19. Decoster L, Van Puyvelde K, Mohile S, Wedding U, Basso U, Colloca G, et al.Screening tools for multidimensional health problems warranting a geriatric assessment in older cancer patients: an update on SIOG recommendations. Ann Oncol. 2015;26:288-300.
20. Reijven P, Sundaram L, Vroomen A, Brouns J, Geerlings P. MON-P194: A short nutritional assessment procedure (SNAP) for routine dietetic practice. Clinical Nutrition, 2017;36: S250.

\section{NOTES}


Een technologische innovatie voor het behoud van het pre-en postoperatief fysiek functioneren vereist ook sociaal-culturele innovatie om duurzame inbedding ervan in het leven van de patiënt en/of de professional te bewerkstelligen. 
Chapter 7

General discussion 


\section{PREFACE}

Globally, health care - specifically also perioperative care - is in transition $[1,2]$ thereby evolving towards components of the concept of predictive, preventive, personalized, and participatory (P4) health care [3]. Small niche experiments are currently being performed, emerging from variations of collaborations between citizens, researchers, the government, and industry [4]. Technological and social innovations - solitary or intertwined - 'compete' with the current practice for a gradual change in perioperative care. At the same time, the "science in transition" movement is emerging from an international discussion concerning research waste [5-8]. Along this transition movement, "Mission-oriented innovation and research" is advocated [9]. The aim of this mission-oriented thesis was to guide the transition in perioperative physical therapy care focusing on maintaining or even improving physical functioning during a major life event episode, especially in frail patients undergoing elective hepatic or pancreatic resection. Several 'point' solutions - state-of-the-art scientific research, theories, and perspectives about proactive perioperative physical therapy management - were explored by an embedded scientist, and implemented in daily clinical practice and combined with a meticulous monitoring and evaluation process of professional staff actions and patient outcomes.

In this chapter the main results of this thesis will be discussed and their social and technological readiness levels (SRL and $T R L)[10,11]$ will be estimated (see Table 7.1 ). At last, the clinical implications and future perspectives on the on-going transition in perioperative care of physical therapists will be presented and discussed, ending up in recommendations for patients, clinicians, researchers, industry, and policymakers involved in perioperative care. 


\section{MAIN RESULTS AND INTERPRETATION OF STUDY FINDINGS}

An embedded science model in this thesis was used to initiate, develop, observe, monitor, and evaluate an ongoing transition in perioperative care management, with the focus on physical functioning and postoperative outcomes for patients with hepatic and pancreatic cancer during their entire treatment course. To assess the extend to what development-stage the results of the studies performed in this thesis have come, the SRLs and TRLs for the instruments and services were determined (both on scales from 1 to 9; see Table 7.1). SRL reflects to what extend knowledge and tools are suitable to integrate in the specific societal system and defines the transition that is necessary for an innovation to acquire societal adaptation [10]. TRL focuses on the sequential stages of development of technological tools and innovations [11].

The research described in this thesis concerns in general two research strategies (see Figure 7.1). The first part (Chapter 2 and Chapter 3) consists of descriptive research. In Chapter 2 , reported current physical therapy clinical practices in Dutch hospitals (response rate 79\%) were evaluated for their compliance to the state-of-the art evidence. Chapter 3 described the initiation and development of a transition towards that state-of-the-art evidence practice in an University Medical Center. Both studies documented an overall initial lack of adoption of state-of-the-art perioperative physical therapy. The initiation and development of the transition towards state-ofthe-art evidence of pre- and postoperative physical therapy at the Maastricht University Medical Center demonstrated the many difficulties of adoption on the one hand (Chapter 3). On the other hand, the study demonstrated some first favorable results in the development and use of stateof-the-art innovative tools and procedures that could help hospital physical therapists in the near future to start adopting another more evidence based working mode. The second part (Chapter 4, Chapter 5, and Chapter 6) focused on research concerning the development and ecological (i.e. in the relevant real-life clinical physical therapy practice) validation of the tools and procedures, that were subsequently introduced in the transition process in the Maastricht University Medical Center as described in Chapter 3. These studies mainly focused on improvement of the TRLs of current preoperative risk assessment tools and exercise prehabilitation procedures for patients that opted for hepatic and pancreatic surgery, as can be concluded from the overall comprehensive overview of this thesis in Figure 7.1. The research investments in these chapters on the TRLs also had implications in the sense of progressive encouraging shifts on the SRLs, as indicated in Figure 7.1. 


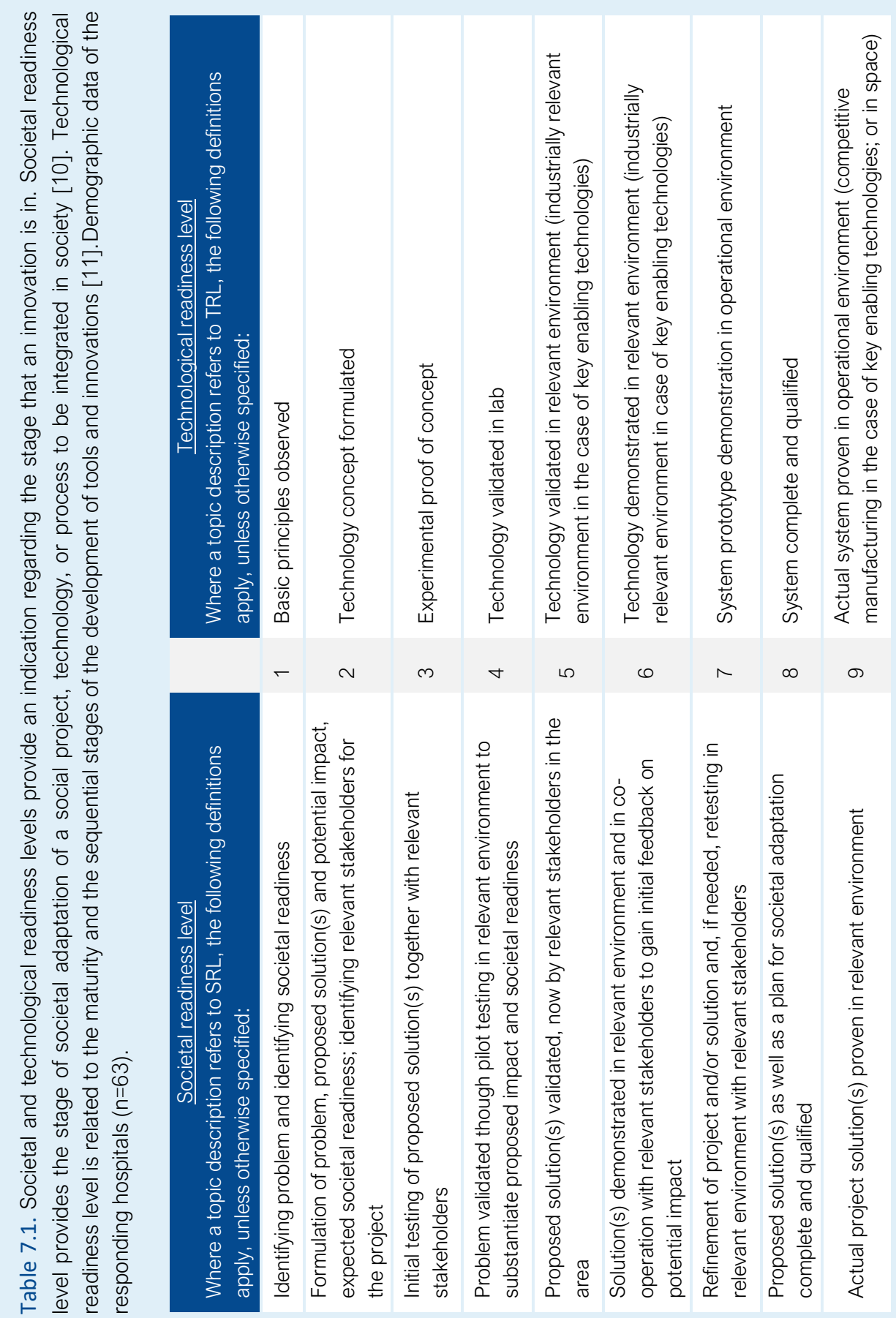




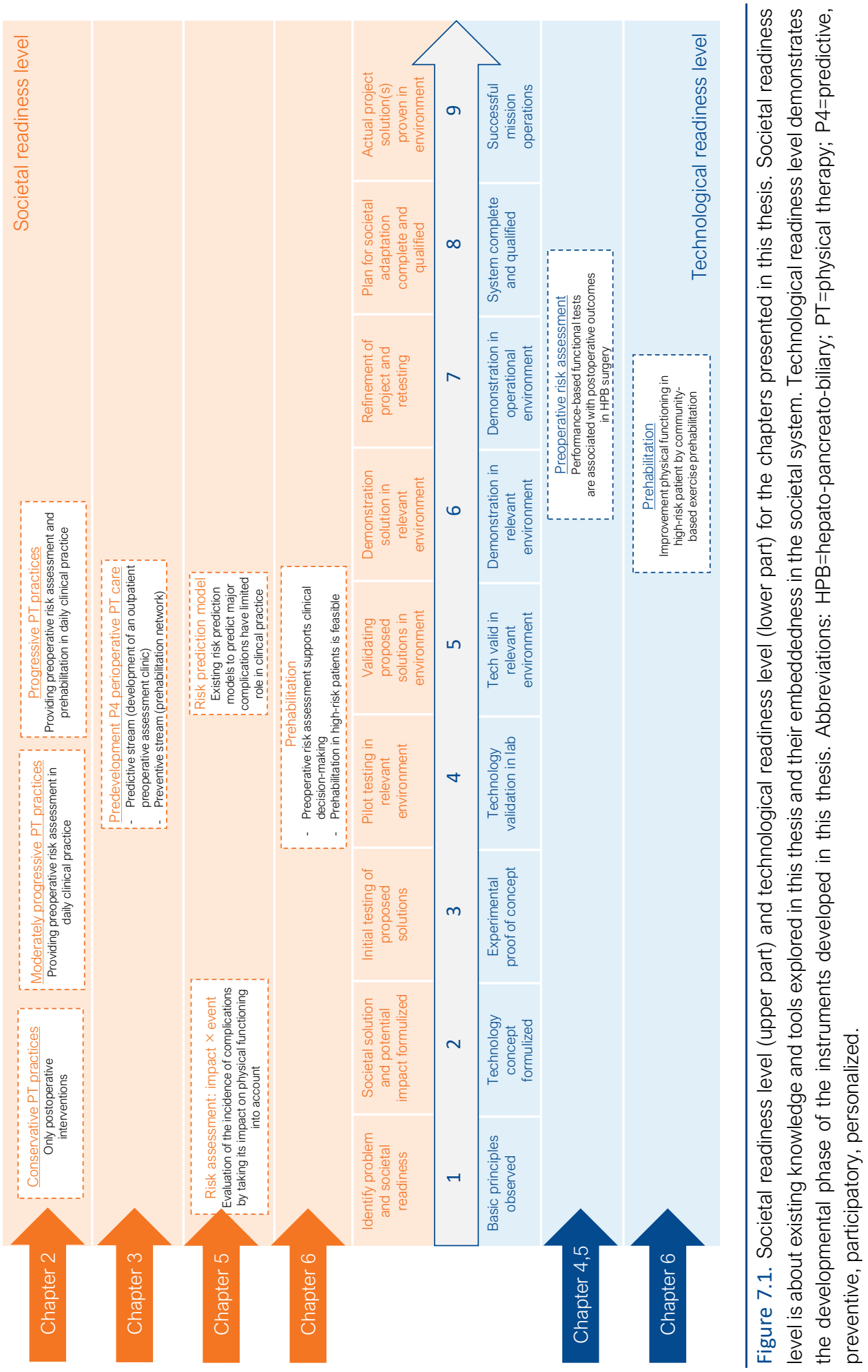




\section{Societal readiness level}

\section{National status of the transition in perioperative physical therapy care}

A cross-sectional survey concerning perioperative physical therapy management for major elective abdominal surgery was performed between 2016 and 2017 in 65 Dutch hospitals with a response rate of $79 \%$. The study was performed to describe the overall reported content and between-hospital variability in perioperative physical therapy management, as well as to examine differences between the nowadays-advised state-of-the-art perioperative physical therapy and the reported perioperative physical therapy in the Netherlands (Chapter 2). The between-hospital variability of perioperative physical therapy management in Dutch hospitals ( $n=65,79 \%$ ) was extensive. From each subdomain of the survey ( $n=4$, preoperative, postoperative, discharge, and readmission physical therapy characteristics of each hospital), 19-items were extracted that corresponded with the advised state-of-the-art physical therapy. Based on differences between these advises and the reported clinical practice, three classes of perioperative physical therapy practice were identified. Class I (progressive, 25\%; frontrunners that correspond to a good adoption state of state-of-the-art perioperative physical therapy) and class II (moderately progressive, 29\%; corresponding to a moderate adoption state) both provided preoperative risk assessment (proactive predictive system). Class I and class II differed in providing prehabilitation (proactive preventive system), which was merely included in class I. Class III (conservative, 46\%; corresponding to a low adoption state) mainly focused on postoperative rehabilitation (traditional reactive system). When calculating index scores for each hospital on the 19-item dichotomous scale, only one hospital $(1 / 63,1.6 \%)$ reported to provide perioperative physical therapy care management corresponding to $75 \%$ of the items on the 19 -items dichotomous scale reflecting advised state-of-the-art physical therapy. In sixteen hospitals (16/63, 25.4\%), the reported perioperative physical therapy corresponded with more than $50 \%$ of the items on the 19 -items dichotomous scale. Even though the data concerns reported perioperative physical therapy practices, one might imply that there is a large gap between current clinical perioperative physical therapy practice and the advised state-of-the-art physical therapy management.

There was a large variability between the different hospitals and a large gap between reported and advised perioperative physical therapy, of which the instruments and services can be scored at TRL 6-8. As such, it might be questioned what SRLs these advised instruments and services may have, as these seem hardly known. Respondents in class III have adopted none of the preoperative tools in current clinical practice, perhaps indicating a low SRL level. The probably low estimated SRLs may indicate the importance of reaching consensus between hospitals on 
optimal perioperative physical therapy management, as well as the need to consider a pragmatic strategy to accelerate and improve (de)implementation in current perioperative physical therapy care according to current state-of-the-art evidence. Especially hospitals in the progressive class (class I) should initiate a debate on a national level to reach consensus regarding performancebased tests to be used in optimal preoperative risk assessment, the content of exercise prehabilitation, and monitoring and (standardized) registration of patient-related outcomes in terms of physical functioning in a national database. Large datasets, including both medical and physical functioning data of different hospitals, may assist in quality improvement via mirror and benchmark information and strategies based on hospital's own and collective data with help of single subject approaches and continuous comparative effectiveness research $[12,13]$. The more progressive hospitals can educate moderately progressive and conservative hospitals about the available evidence and its impact upon physical functioning of patients. In addition, these frontrunner hospitals may be able to provide de-implementation and implementation strategies to optimize perioperative physical therapy management based on their own trajectory of quality improvement of perioperative care.

Clinical implication I: Alignment on a national level in the profession concerning perioperative physical therapy management is necessary to compose a formal strategy including a guideline - to improve a patient's physical functioning before and after hospitalization and surgery

Suggestion: Physical therapists can learn from the Dutch Federation for Medical Specialists (FMS) and their visitation and accreditation strategies in Dutch hospitals. A likewise installed hospital physical therapy department visitation and training accreditation program might be developed to stepwise learn, develop, and improve physical therapist's perioperative services nationwide.

\section{Reported clinical practice $\neq$ real-life clinical practice}

The response rate of the survey was $79 \%$, which made the survey rather representative for the current Dutch perioperative physical therapy care management in patients undergoing colorectal, hepatic, or pancreatic resection. It was encouraging that $17.5 \%$ of the respondents reported to provide exercise prehabilitation in these patients, and that several progressive practices were identified based on the 19-item dichotomous scale concerning advised state-of-the-art perioperative physical therapy. However, the survey collected self-reported information and might 
therefore lead to bias by not directly reflecting real- life practice and providing socially preferred answers. Furthermore, generalizability of these research results, clinical implications and suggestions towards physical therapy practices in other countries and health care settings might be difficult due to cultural differences.

Practical consideration I: Self-reported data on current perioperative physical therapy practice might not provide an accurate reflection of daily clinical practice. The collection of true observational data and the use of a clinical outcome measure database on a national level might provide better insight in clinical perioperative physical therapy practices and their impact on physical functioning of patients during their surgical trajectories.

Suggestion: Combine observational perioperative physical therapy data with data from the Dutch clinical registries, such as the Dutch Institute for Clinical Auditing. This may assist in providing insight in a patient's physical functioning in different hospital settings. Based on these data, a stepwise program of quality improvement via mirror and benchmark information might be induced.

\section{Local transition in perioperative physical therapy care}

At the niche level within the Maastricht University Medical Center the transition towards a proactive perioperative care pathway for patients opting for elective hepatic or pancreatic resection was performed in multiple stages, with continuous monitoring of qualitative and quantitative data (Chapter 3). This transition experiment was guided by an embedded scientist, who aimed to connect the P4 perioperative care streams (predictive, preventive, personalized, and participatory [14]) amongst actors in the system (here: the hospital and its region) in order to create alliances on operational level to change practices in the system. The results of this study can be classified at SRL 4-5, as predictive and preventive care solutions with a TRL of 6-8 were validated in the relevant real-life clinical context. These predictive and preventive care solutions aim to determine and prevent a patient's potential perioperative risks for a delayed recovery or even permanent loss of physical functioning. Nowadays, outpatient preoperative assessment of a patient's physical fitness and physical functioning, exercise prehabilitation, and daily monitoring of a patient's postoperative recovery of physical functioning are an integral part of perioperative physical therapy care pathway for patients undergoing hepatic or pancreatic resection in Maastricht University Medical Center. The adaptive partnership between patients, health care professionals, and researchers within this embedded science approach allowed for a co- 
production in exploring new forms of knowledge, values, and social relations, and thereby narrow the gap between research findings and real-life clinical practice $[15,16]$. This collaboration allowed for the development of technical instruments (test battery of practical performance-based tests of physical functioning) to demonstrate the significant association between preoperative level of physical functioning and short-term postoperative (TRL 6-8; Chapter 4 and Chapter 5). Chapter 6 illustrated how preoperative risk-assessment can support clinical decision-making in a high-risk frail patient opting for major abdominal surgery in the real-life clinical context. In addition, Chapter 3 demonstrated that the adaptive academic partnership was valuable in developing societal impact via a reduction in the median hospital length of stay (a reduction of 4 days in pancreatic resection and 1 day in hepatic resection) and a reduction in the median time to recovery of physical functioning (a reduction of 2 days in pancreatic resection and 1 day in hepatic resection).

Of course, these results do not provide a clear and undisputed cause-effect relationship between all interventions and the results. Thus, one could argue about many of the components of this whole trajectory, as for instance the embedded science model used in this thesis. Randomized clinical trials might shed more clear light in the cause and effect relationship and are still the dominating research design for evidence-based research. However, it is questionable whether this is the appropriate research method when considering the lack of generalizability to real-life practice in an evolving era of personalized medicine and rapid technological adoption [17, 18]. The embedded science model used in Chapter 3 corresponds to the idea of continuous single subject approach (for mirror data) and options of single subjects to step into a multiple single subject approach, which in essence is the continuous comparative effectiveness research approach [19]. Single-subject and comparative effectiveness research both comprise continuous monitoring of one or more systems to evaluate all sorts of parallel and sequential health care and health care related interventions in systems and relate these to results that are collected by or with patients and clinicians throughout the patient's perioperative journey in a real-life context. This will generate evidence that allows for comparison of alternative treatment options with the intention to make well-informed decisions at both the individual and population level [19], both in single and multiple hospitals. Moreover, it enhances and guides technological innovations [20]. The experiments as presented here may be seen as part of an nowadays ongoing paradigm shift from evidence-based research to context-based practice [21]. At the same time, the real-life context approach - the setting where care is received by the patient and provided by the professional - address the more recent insights that the effectiveness of interventions is substantially more codetermined by context than was inferred in the years before, and that 
context influences especially the so ecological validity of research results [21]. The context was and sometimes still is typically seen as providing "background noise", which makes it difficult to observe the "signal" (the key variable of interest). To reduce the noise or to enhance the signal in clinical studies, the set-up of an intervention and its context components are normally strictly controlled in the study design. Additionally, the patient population involved is than mostly assembled under (strict) in- and exclusion criteria. All these phenomena were and still are formerly considered as 'non-essential' issues of research, which however happen to become more and more known as being quite essential in hindsight. Randomized clinical trials left physical therapists with tools and services with relatively high TRLs, but with low SRLs. The low SRLs are due to the fact that adoption and implementation in practices is lacking as can be seen from the results of Chapter 2, in which for example the use of risk assessment instruments and prediction rules in Dutch perioperative physical therapy practices is scarce $(n=20 / 64,31.7 \%)$. So, despite the fact that a causal relationship can be established with a high internal validity by using for instance a randomized controlled trial, it leads to a more or less artificial 'laboratory' context and generally generates a large discrepancy between the research context and the day-to-day real-life context. Hereto, researchers are on the brink of new ways in which clinical research maximally improves TRLs and SRLs, thereby allowing cause-effect attributions at the same time. Even more importantly, the latter benefits patients while simultaneously creating social and scientific value [22-24].

Clinical implication II: "Context matters". Integrating research in daily clinical practice provides a way to build a sustainable adaptive academic partnership for continuous monitoring and improving health care interventions throughout the patient journey in a reallife context. New methodology-approaches provide means to elaborate on cause-effect relationships

Suggestion: Invest in embedded researchers who act as 'knowledge brokers' to initiate, develop, and implement evidence-based tools and services in the context of the hospital. Thereby aiming to improve TRLs and SRLs, preferably with state-of-the-art methodological approaches allowing for deliberations on cause and effect relations. 


\section{Social deprived system and establishing effectiveness}

For further explanation of the results discussed so far in this chapter, an insight stemming from physics might be of value here. In physics namely, it is generally considered that a system always aims to achieve a preferred and most efficient mode. When viewing the role of a system in a dynamic landscape with "hills" and "valleys", the system (here the perioperative physical therapy system in a hospital and its region) will settle in a valley with a relatively low energy potential (see Figure 7.2). External influences define the relative stability of the system and when a small perturbation is applied to the system, it will move away from its original state (valley). Depending on robustness of the original state and strength and length/duration of the perturbation, the system might move and climb a hill, which costs a lot of energy (in order to cope with the resistance of the current system). Ultimately, this might lead to either a fall back of the system in its former valley, or to a new gradually build-up of a steady state system in a completely new and better suited valley.

The concept described in Figure 7.2 relating to the dynamic system theory can be applied on the transition experiment described in Chapter 3. It was hypothesized that perturbations were caused by the initiation of diverse experiments to improve a patient's physical functioning throughout the perioperative period. Consequently, these perturbations will drive the perioperative system away from its stationary state (valley). However, there is no evidence that the observed substantial difference of the dynamics of the system can be attributed to the transition experiments, or whether it is an epiphenomenon of the organization of health care as a whole. Furthermore, based on the sedentary environment in the hospital - like in many hospitals $[25,26]$ - without the focus on a patient's physical functioning, any intervention might probably have influenced the relatively stable inferred socially deprived system. As there was no direct attention for a patient's pre- and postoperative physical functioning and in-hospital physical activity levels were low, even small interventions might influence a patient's recovery of physical functioning. Consequently, for evaluating the effectiveness of the diverse interventions while simultaneously improving daily clinical practice, the continuous monitoring of culture, practice, and structure and stepwise implementation of new practices and procedures is necessary to change the current system and thereby hopefully also its performance. At least, that is what we infer and consider plausible. Therefore, it is advocated to perform continuous monitoring of relevant outcome parameters at both a patient and system level, and to intervene based on suboptimal outcome data of patients (preferably their performances) and - later on - costs (preferably value-based health care). In this way, the (embedded) researchers and physical therapists have the ability - but more importantly 
the moral and legal obligation - to improve the standards of daily clinical practice and act upon the changing society and economy to anticipate on the increasing demands towards the health care system.

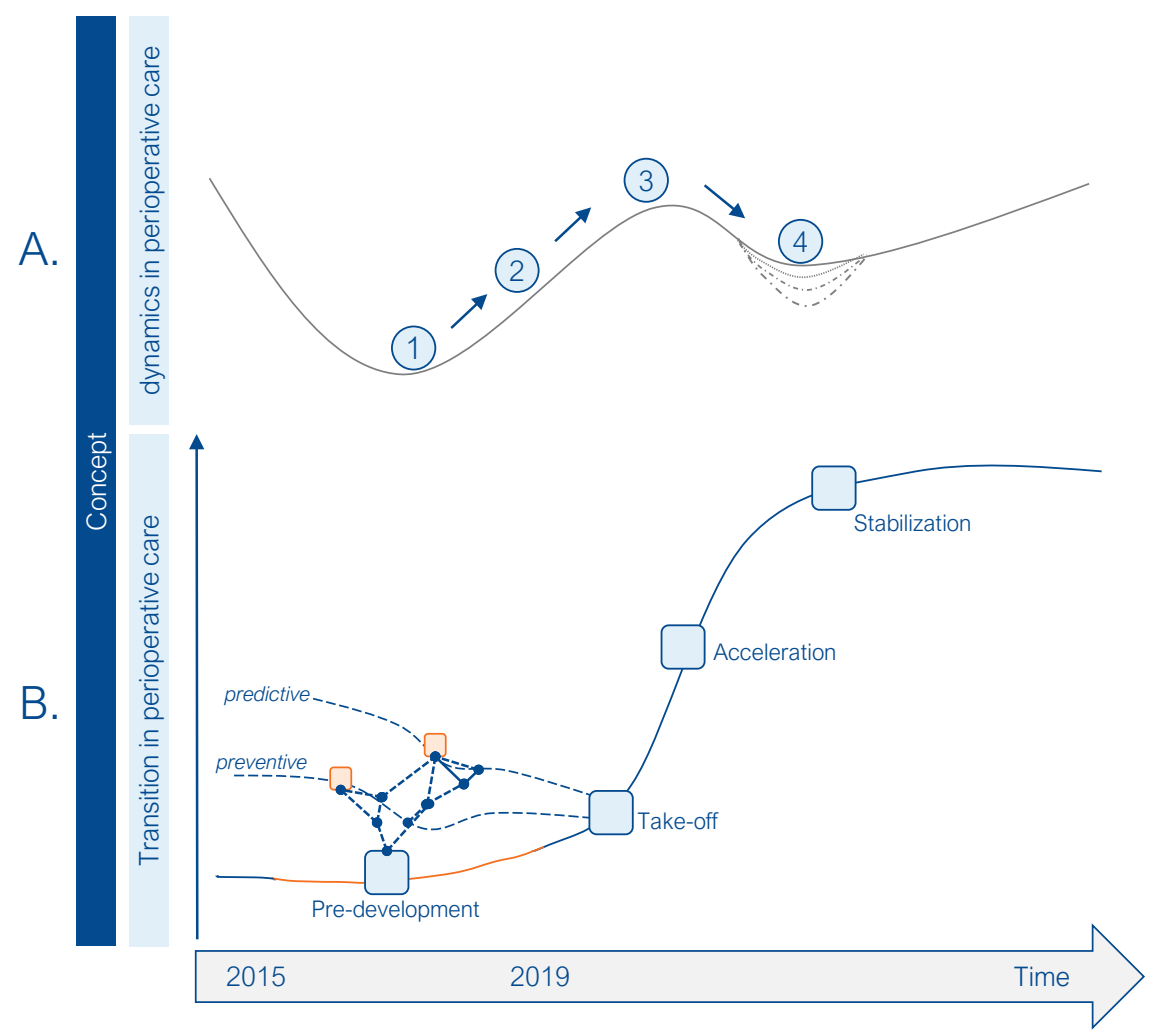

Figure 7.2. The concept consists of the dynamics of the perioperative care system and the transition in perioperative care.

A. The relatively stable perioperative care system is represented as a ball in a valley (1). Depending on the steepness of the valley, small perturbations will push the ball uphill (2). Given enough time and perturbations that are strong enough, the ball will probably be pushed on top of the hill (3). Next, the ball might fall back towards the old system when the perturbations are not strong enough (1) or might encapsulate itself in a new valley, a new and optimized (stable) state of the system (4).

B. The transition in perioperative care, which is characterized as a non-linear process divided in different phases (pre-development, take-off, acceleration, and stabilization phase) with slow and rapid changes. The perioperative care system $(\square)$ is changing due to different actors collaborating in different perioperative care streams (e.g., predictive, preventive ----) leading to the emergence of a singlesolution system $(\square)$ in the perioperative care system.

$\mathrm{A} \times \mathrm{B}$. The ball at the bottom of the steep hill requires strong attractor(s) - changes in culture, structure, and practices by different professionals - to shift it towards the top of the hill or to move it to a new stable system. Professionals in the upcoming streams (e.g., predictive, preventive) will develop specific small-system solutions contributing to the overall transition of the perioperative care system. 
Practical consideration II: An iterative cycle of interventions in the system - here the hospital - and simultaneous data collection of relevant performance indicators of the system and of patients that should be analyzed, and reflection upon these, is essential to monitor the transition of a system in a dynamic landscape and to monitor the essential hereby warranted changes of outcomes for patients entrusted to the system.

Suggestion: Patient profiling models provide a rational approach to allocate the treatment resources. Learn from the frontrunners on these techniques, for instance Lutz [27], and associate with the national data-infrastructure and technology [28].

\section{Technological readiness level}

\section{Preoperative risk prediction}

Preoperative risk stratification to identify patients who are at risk for adverse surgical outcomes often relies on a classification system with non-modifiable conventional risk factors, such as age, American Society of Anesthesiologist (ASA) grade, and comorbid conditions [29-31]. Cardiopulmonary exercise testing (CPET) to assess a patient's preoperative aerobic capacity is valuable to identify patients that have an increased risk for adverse postoperative outcomes [3235]. Based on the evidence of CPET, we consider its TRL as rather high: TRL 6-8. However, performing CPET is not always feasible in daily clinical practice as it requires special equipment, trained staff, it is relatively time-consuming. Consequently, the SRL for daily practice of the CPET might be valued as 2-4/5. Based on literature and clinical experience, it was hypothesized in this thesis that in patients with cancer in the liver or pancreas, like in many other cancer types, the preoperative level of physical functioning measured with practical performance-based tests (preferably with high TRLs and SRLs) was associated with postoperative outcomes. Those practical performance-based tests could be used to evaluate perioperative risk in patients undergoing hepatic and pancreatic resection (see Chapter 4 and Chapter 5). Findings demonstrated that - besides conventional patient-related medical and demographic risk factors as respectively ASA grade and body mass index - the performance of a patient on practical performance-based tests of physical functioning was associated with short-term postoperative outcomes (see Figure 7.1, TRL 6-8). Especially, estimated aerobic capacity (steep ramp test performance) was associated with postoperative complications following both hepatic and pancreatic resection. Moreover, estimated aerobic capacity (steep ramp test performance), functional mobility (two-minute walk test performance), perceived level of functional capacity to perform activities of daily life (veterans-specific activity questionnaire and Duke activity status 
index score) were independently associated with a delayed recovery of physical functioning measured with the modified version of the lowa level of assistance scale (mILAS) in patients following hepatic and pancreatic resection. These results, in addition to those of others [36, 37], emphasize the importance of an adequate level of physical functioning before major elective abdominal surgery to reduce the risk for adverse surgical outcomes. The research presented in Chapter 4 and Chapter 5 provide elements of TRL and SRL improvement in het context of the Maastricht University Medical Center in patients with cancer in the liver or pancreas. Practical performance-based tests of physical functioning are also used in other contexts [36-38] as they posess the proper TRLs and SRLs. Hence, these tests can be relatively easy used - after an educational course - by other hospital physical therapists in the Netherlands and abroad.

Early preoperative identification of modifiable risk factors provides the opportunity to timely recognize patients that are at risk for adverse surgical outcomes (e.g., delayed recovery of physical functioning, morbidity). For these patients, targeted preventive strategies should be initiated with the aim to preoperatively improve a patient's capacity to cope with the stressors of hospitalization and surgery. Numerous preoperative prediction models have been published. However, their applicability and role in clinical practice, as well as data concerning their accuracy are often lacking [39]. Concerning the prediction of relevant clinical outcomes, currently available risk prediction models mostly use the incidence of postoperative morbidity as primary outcome. However, in an explorative analysis of 27 patients experiencing a major complication following pancreatic resection (Chapter 5), the range in preoperative level of physical functioning in patients experiencing a major postoperative complication was broad. For instance, aerobic capacity estimated from steep ramp test performance ranged from $0.92 \mathrm{~W} / \mathrm{kg}$ to $4.12 \mathrm{~W} / \mathrm{kg}$. When also taking the impact of a major complication per patient into account by looking at the time to recovery of physical functioning, those patients with a higher preoperative level of physical functioning seemed to cope better with postoperative major complications. Patients experiencing a major complication without a delayed recover of physical functioning had a significantly higher steep ramp test performance $(2.89 \mathrm{~W} / \mathrm{kg})$ compared to patients experiencing a major complication with a delayed recover of physical functioning $(2.07 \mathrm{~W} / \mathrm{kg})$. Given these observations and recommendations in a recent systematic review of Thomas et al. [40], it is advocated to explore and validate alternative outcome measures for evaluating the "event $x$ impact" construct for all sorts of pre- and postoperative complications. Additionally, other (longterm) patient-centered outcome measures that focus on physical functioning should be developed when evaluating the impact of postoperative complications. 
Clinical implication III: Early preoperative evaluation of a patient's physical functioning when opting for hepatic or pancreatic resection is valuable to establish a patient's adaptive capacity to cope with the stress throughout the patient-journey. It is advocated, based on this thesis, that it should be an integral part of the conversation between the patient and the professional in clinical practice for decision-making.

Suggestion: Not only 'just' register the event of a complication per patient per hospital, but combine the event with its inferred impact, for instance by combining an event with time to recovery of physical functioning (measured with the mILAS) or hospital length of stay. Incorporated this event $\mathrm{x}$ impact in mirror and benchmark informatics and trial statistics.

\section{Association $\neq$ prediction}

The results in Chapter 4 and Chapter 5 demonstrated the importance of an adequate level of physical functioning before surgery in patients undergoing hepatic and pancreatic resection. However, prediction of the probability that an individual will experience a certain "event" (e.g., postoperative complication) was not possible in the period of this project because of the lack of adequate power (too few events per variable during the project years). For determining associated variables, a less stringent alpha value was used for pre-selection of relevant variables $(P<0.20$ instead of $P<0.05)$. The results might be difficult to interpret, as the reader is mostly used to look at an odds ratio and its 95\% confidence interval; in our results, the odds ratio and its 95\% confidence interval may overlap the null value, because of this less stringent alpha value. However, the pre-selection of variables with an alpha of 0.20 is also suggested by prediction modelling guidelines [41] in order to prevent valuable variables to be removed too early from a model. The current set of variables used in logistic regression analyses in Chapter 4 and Chapter 5 were selected based on clinical experience and a review of the literature. Hence, P-values are useful but should not be the yardstick to decide which type of variables should be included in the model.

Sarcopenia, along with other clinical factors, is a commonly used marker for frailty and used, mainly via the use of certain biomarkers [42], to identify patients at risk for one-year mortality after gastrointestinal surgery for malignant conditions [43]. It would be interesting to combine the variables of performance of physical functioning from our study with the identified (bio)markers of sarcopenia. Furthermore, also myosteatosis, other biomarkers and personal factors, such as coping strategy and expectations were not considered. Those variables might also mediate 
and/or moderate a patient's recovery of physical functioning, length of hospital stay, and risk for complications. Associations were only explored for short-term postoperative outcomes (30 days), and a patient's physical functioning was not measured after 60 or 90 days postoperatively. For long-term effects, there is mainly a focus on recurrence of the disease and mortality, but physical and social consequences of the disease and its treatment on a patient's experienced health related quality of life should not be underestimated.

Practical consideration III: In a small dataset or in a dataset with few events, the development of a risk prediction model seems often not feasible per disease/surgical procedure. When considering the development of a risk prediction model, other factors like measures of frailty should also be taken into account. Alternatively, groups of patients can be combined in which the emphasis will lie on physical functioning rather than the disease or (surgical) procedure [44].

\section{Prehabilitation}

The case report in this thesis (Chapter 6) illustrated the care pathway of a high-risk patient who opted for pylorus-preserving pancreaticoduodenectomy (PPPD), including preoperative optimization for this procedure by participating in a community-based exercise prehabilitation program. The case report described preoperative patient examination, evaluation of the results in decision-making for surgery, the prehabilitation program, and postoperative outcomes with help of the recommended Hypothesis-Oriented Algorithm for Clinicians II (HOAC II) for physical therapists. Although a risk prediction tool was at the time of executing this case study not ready and available, the utilized preoperative multidisciplinary risk assessment resulted in an open conversation between the patient and the caregivers team concerning the risk-benefit ratio of the surgical procedure. The latter supported the decision of the patient and her team of caregivers to participate in a prehabilitation program at a community-based physical therapy practice. Thereafter, a four-week prehabilitation program was completed which resulted in a preoperative increase in the patient's level of physical functioning. The surgical procedure and postoperative functional recovery were without any complications.

Formerly, prehabilitation has already been shown to be beneficial in increasing a patient's preoperative level of physical functioning, while authors conclusions concerning its benefits on postoperative outcomes vary from beneficial [45] to inconclusive [46, 47]. A low therapeutic validity of exercise prehabilitation programs, more specifically, lacking preoperative risk assessment to identify the less physically fit patients that will benefit the most from exercise 
prehabilitation, and suboptimal training frequency, training intensity, and/or training time might explain the variation of conclusions [40]. The 'flaws' in these research projects on several prehabilitation approaches have been published $[48,49]$ and new approaches have suggested and are being studied [50]. The case report in this thesis illustrated the importance of an interdisciplinary preoperative risk assessment and shared decision-making with the patient to participate in a prehabilitation program. As there was no formal risk prediction model available for this specific type of the used set of practical performance-based tests was validated already in other surgical populations and was supposed to be quite able to provide insight in this patient's physiological reserve capacity. Before the start of the preoperative training program, the patient was not intrinsically motivated to participate, as the patient stated that she did not like to participate in sports or to use training equipment. However, after the patient was well informed by her team of caregivers about the potential benefits of preoperative physical fitness and physical activity, the patient understood the importance of the prehabilitation program and was motivated extrinsically and willing to participate.

The patient participated in a supervised prehabilitation program at a nearby community physical therapy practice, which was supplemented with functional task exercises the patient performed unsupervised at home. The patient stepwise monitored her physical fitness in joint coalition with her physical therapist and became aware of her gradual improvement of her physical functioning preoperatively. The executed prehabilitation program in this patient can be classified at SRL 4-5, with a high therapeutic validity (seven out of nine items of the CONTENT scale). However, the SRL level is still behind the TRL level of prehabilitation that is already established at TRL 7-9 as described below. It is advised and feasible to execute the exercise prehabilitation at home to overcome problems with adherence, motivation, or lack of transferring training skills to the homebased setting. By doing so, a more physically active complete continuum from the care context to the home context and vice versa is created. Such a home-based physical exercise program was shown formerly to increase the participation rate of high-risk patients, as these patients are often unwilling or unable to participate in a hospital-based prehabilitation program due to all types of practical limitations as transport, informal caregivers support and job- or urgent home front obligations [50-52]. This way, physical therapy is also provided in the right mental, social, and physical context when provided at the patient's home context, as the training environment closely matches the challenges that high-risk patients encounter during daily activities [53]. The patient and physical therapist can adjust training to personal situations, considering the physical fitness level of the patient and affinity with the functional exercises. Moreover, the living environment can offer a safe environment and can be very useful when a partner or family and/or friends are 
present during training to offer support and continuity, even without the presence of a physical therapist as the main attractor of the training execution of the patient. The overall goal is to preoperatively improve a patient's adaptive capacity by improving cardiovascular, respiratory, and musculoskeletal parameters to cope with stress of hospitalization and surgery. Therefore, adequate monitoring of these parameters in the home-based setting preoperatively within the relevant daily tasks of the patient is important. This way, training progress can be frequently evaluated, and patient-specific exercises can subsequently be progressed on intensity, repetitions, complexity, and variability to continue providing an adequate training stimulus. Moreover, it can then be decided whether a patient is shifting from a high-risk profile towards a low-risk profile and achieved the optimal level to adapt to the stressors of hospitalization and surgery $[54,55]$. As well as if a patient can cope with the complications and have the ability to go home and resume activities of daily life on their own.

As said already before, the benefits of exercise prehabilitation on postoperative recovery are shown in several groups $[45,56]$ but also have been reported as being inconclusive or absent in others $[30,38]$. However, this variation cannot be explained completely by the aforementioned lack of an appropriate context and content of preoperative physical therapy. Additionally, we have to bear in mind that currently bed-centered care is still a hospital's and patient's main recovery strategy, which hampers a patient's recovery of physical functioning [57]. To optimally make use of the potential effects of prehabilitation, the activating home-based context and therapy should also be provided along and during hospitalization. Furthermore, as explored in Chapter 5, the impact of a postoperative complication might be influenced by a patient's resilience to cope with the stressor. A fit patient, with sufficient adaptive capacity, might not experience much impact of a complication on the level of physical functioning, while an unfit patient might experience severe health issues requiring intensive care and thereby significantly impairing the patient's level of physical functioning. Hulzebos et al. [56] have shown these differences in patients with postoperative pulmonary complications after coronary artery bypass graft procedure. When interpreting the effectiveness of prehabilitation in terms of reducing complication rates, its effectiveness might sometimes be hard to establish, as it may partly be due to type of surgery, presence of unmodifiable risk factors, locally advanced tumours or involvement of additional organs $[29,58]$. Hereto, the effectiveness of prehabilitation should besides being evaluated on complication rates, also being evaluated on the impact of a complication on a patient's physical functioning in relation to the stimulating context during the entire trajectory. Furthermore, a costbenefit analysis should be performed to establish whether there is a solid return of investment of prescribed preoperative physical therapy. The costs of preoperative risk assessment and 
prehabilitation in (high-risk) patients should be compared with savings of an accelerated recovery of physical functioning and its associated reduction in health care costs when performed under a strict innovative regime as described above. Cost-effectiveness analysis require high TRLs and SRLs of tools and innovations and thus are of rather easy use and should be implemented in physical therapy practices.

Clinical implication IV: Prehabilitation is feasible and effective in most and probably all patients with an established high-risk profile. However, it should not merely consist of preoperative exercise training for all patients but should at least include preoperative risk stratification to identify the right patients who benefit the most from prehabilitation. Furthermore, personalized home-based functional exercise training, adequate objective monitoring of training progression, and providing a physically activating context and culture throughout the continuum of care are necessary to establish beneficial effects of prehabilitation.

Suggestion: Develop and implement a mandatory, evidence- and practical-based decisionsupport system for patients, their relatives, and caregivers, as well as for financers and policymakers that provide insights in the trajectory and tools to use for optimizing a patient's physical fitness and physical functioning throughout the perioperative trajectory and that

\section{$\underline{\text { The value of (multiple) case studies }}$}

As the cause-and-effect relationship between prehabilitation and its beneficial effects on postoperative outcomes in trials with a high therapeutic validity has already been established [45, 56], researchers should look for using other research designs to sustainably implement and evaluate prehabilitation in the right setting, for the right patients, using the right therapy, and right outcomes measures [40]. Here we tend to speak of "ecological" validity, strictly focused on the setting, patient, and moment at hand. Experimental designs, like randomized controlled trials, are not necessarily "better" than non-experimental designs, as the latter could provide more insight in the real-life context or demonstrate why some patients show better responses to prehabilitation than others. There are no inappropriate methodological designs; however, there are designs inappropriately used and researchers should keep in mind the following items when choosing for a certain design for their research: why, what, for whom, and which setting [59]. 
In the light of more personalized and tailored health care, focusing on individual patients and their specific characteristics and needs is more personalized than assigning patients to a group and give them the same treatment. When studying a single patient, close monitoring - ideally with the use of low cost, high impact technology - of relevant outcome measures is easy and therapy can be adapted easily (e.g., the concept of titration) [60]. Multiple single case studies have the ability to explore variability within patients receiving a certain treatment. Over time, patient characteristics or response profiles might be obtained when comparing data of individual patients.

Practical consideration IV: Although it is hard to establish a cause-and-effect relationship within a single case, it focuses on the optimal treatment for a single individual. However, by comparing multiple single cases, more homogeneity in the response on treatment might be observed among patients based on certain common patient and intervention characteristics.

Suggestion: Train patients and physical therapists to monitor, evaluate, and act upon the results of their measured level of physical fitness and physical functioning. People are more and more already interested in their own (health) data [61] but physical therapist should be trained how to use the patient's and the clinical data in combination in order to improve the performance of their patients, themselves and of the profession as a whole (decisions for therapy initiation, titration, and cessation) $[62,63]$.

\section{FUTURE RECOMMENDATIONS FOR RESEARCH AND CLINICAL PRACTICE}

The transition towards P4 perioperative care for patients that need HPB surgery is currently (locally and (inter)nationally) in the pre-development and pre-experimental phase. The results of this thesis contribute, besides other well-known developments in the perioperative field [64, 65], to more broadly seen changes in perioperative care. As perioperative care is of paramount importance and at the same time rather complex, the results of this thesis should not 'just' be perceived as an isolated part offering a solution for the loss of physical functioning during a major life event such as surgery. When looking at this thesis, one should bear in mind the parable of six blind men touching an elephant; without connecting the six subjective experiences, their individual experiences have almost no meaning at all. However, when combining these six experiences, one gets the 'feeling' of an elephant. In parallel one should probably see perioperative care likewise; it is important to understand and connect all involved parts in order to completely understand the system, to improve it, and thereby to improve a patient's physical 
functioning throughout the perioperative period. In the following paragraphs, recommendations for future research and clinical practice will be discussed in order to improve the perioperative system on a local and (inter)national level.

\section{Local}

The initiation of transition towards P4 perioperative physical therapy care in a single academic hospital demonstrated that a health care transition is dynamic and complex, thereby demanding multiple orchestrated combined strategies, and requires alignment with multiple actors (Chapter 3). Following the P4 perioperative physical therapy streams (Chapter 3), the predictive, preventive and the personalized streams were most developed and connected to the daily clinical practice. This resulted in the recognition of the importance of preoperative physical functioning in relation to short-term postoperative outcomes, revealed that preoperative physical functioning is a modifiable factor, and demonstrated that patients might benefit from preoperative interventions to improve physical functioning. Despite the lack of an already entirely validated specific risk prediction tool for patients with cancer in the liver or pancreas, medical and functional preoperative risk stratification is recommended. Preoperative risk stratification is needed to timely identify patients who are at an increased risk for adverse postoperative outcomes and who might therefore benefit from preoperative prophylactic interventions. Future research on determinants that affect a patient's recovery of physical functioning should also include preoperative inflammatory, behavioral, and nutritional parameters, besides conventional preoperative risk factors and the level of physical functioning.

\section{Proactive preparation time}

The generally used term "waiting time" (or "waiting list") for the time between the moment a patient is diagnosed and indicated for surgery and the admission and surgery itself assumes a rather passive and reactive approach of a patient. This "waiting time" is normally advised or even mandated as to be kept as short as possible. However, especially when preoperative exercise training is indicated in patients with an increased risk of a prolonged postoperative recovery of physical functioning (e.g., due to a complication), the passive and reactive "waiting time" period is ideally changed into a proactive preparation time. By doing so, supported by (in)formal caregivers, the patient has the ability to improve preoperative physical functioning to reduce perioperative risks. This implies that the duration of the preoperative period should ideally be determined by weighing up the medical urgency for surgery against the time a patient needs to reach an optimal level of physical functioning when undergoing surgery. To achieve an optimal 
level before surgery, structural changes (logistics, planning of surgery), and practices (close monitoring by the patient's and his (in)formal caregivers of improvement in preoperative physical functioning, in which the training principle "law of the diminishing returns" might indicate an individual patient's optimal physical functioning level) are necessary.

\section{Participatory and personalized (patient-tailored) care}

Participatory and personalized (patient-tailored) care is an approach that implies joined care between health care professionals and patients and his or her relatives, as well as that care should be tailored to a patient's individual needs and potential to reach short-term and long-term goals. In clinical practice however, it is generally considered that it is difficult to realize this approach. However, it is arguable whether it is difficult to realize this approach, or that health care professionals and researchers are lacking the courage to involve the patients, the knowledge, and tools how to realize participatory and personalized (patient-tailored) care. During the transition at an academic hospital, several initiatives were explored to increase a patient's participation (e.g., becoming more actively involved in the decision-making, monitoring of their own recovery of physical functioning). However, these initiatives did not actually improve a patient's participation in the pre- and postoperative period (Chapter 3), whereas the single case study did (Chapter 6). The former might be due to the fact that the initiatives were developed without involving patients. From the literature, it is known that the perspective of patients concerning health care or treatment is different from the perspective of health care professionals [66]. Therefore, it is essential to actively involve the patient in the care (innovation) process, as we did in the case study. Patients, health care professionals, and researchers should work in real partnerships to come up with solutions to improve perioperative care. Furthermore, a hospital context (culture and infrastructure) should be created in which patients are better informed to make well-informed treatment decisions, and in which patients are stimulated to actively participate in their own recovery of physical functioning. For example, throughout the process of decision-making for surgery, patients should be provided with their own health and (physical) performance data to make well-informed decisions about their treatment with involved health care professionals and/or their relatives. Postoperatively, the hospital context should invite patients to be more physically active and to perform activities of daily life, like having lunch in a dining room, when possible. The aforementioned reasons provide arguments why participatory and personalized (patient-tailored) care is feasible and applicable. 


\section{(Inter)national}

The research and innovation projects described in this thesis contributed to the transition towards a proactive $\mathrm{P} 4$ health approach in perioperative physical therapy care and aligned on the mission for perioperative care (Chapter 1). However, a mission implies collaboration with other domains as well (e.g., nutritional, technological, behavioral), and requires creative perspectives and solutions on a societal issue. Therefore, besides advice of a national alignment in perioperative physical therapy care, physical therapists should not be afraid to explore other boundaries than they have already done as well. This was mentioned 20 years ago (in 1999) by Lopopolo et al. [67] regarding the changing role of physical therapists in the acute care setting concerning patient care and professionalism. Within this thesis, the role of hospital physical therapist working in the field of oncological perioperative care might change from a reactive approach towards a proactive approach, focusing on perioperative prediction and prevention, as well as on monitoring a patient's physical functioning throughout the perioperative period in which the patient is in the lead, when possible. Physical therapists should work interdisciplinary (e.g., with other health care disciplines, other research disciplines), cross-sector (nutrition, logistics, data infrastructure), and with cross-actors (e.g., patients, public organizations, private organizations) to develop innovations that align on the mission in perioperative care to provide more personalized and participatory care adapted to a patient's needs to maintain vital physical functioning (see Figure 7.3). It might be of relevance that physical therapists will engage themselves in projects relating to the hospital environment. The demotivating elements of the infrastructure, such as the television above the patient's bed, can be de-implemented and motivating elements close to patient's activities of daily life can be implemented. The infrastructural interventions demand a more orchestrated and robustly aligned effort of professionals, in coalition with hospital management and national branch organizations.

\section{Technology: data-management}

Data on a patient's medical and physical functioning is fragmented in different 'silos' in hospitals (routine clinical data collected at different departments), at research departments (scientific data collected in different clinical trials), and clinical registries, which lack communication with each other. Organizations are experiencing technological, human, and cultural challenges in performing adequate data-management [68, 69]. Therefore, data-driven personalized predictions and decisions for surgery remain complicated, because of the design of our current digital ecosystem. An overarching data-infrastructure is necessary and available (at TRL 4-6 already, but at SRL 2-4 in the professional context of the hospital and its staff) in which patients 
have insight in their health care data and in which cross-sector cooperation in data use between health care professionals and research institutes is facilitated.

Two necessary conditions should be met to achieve an overarching data-infrastructure. First, the data should be arranged following the 'findable', 'accessible', 'interoperable', and 'reusable' (FAIR) principles [70]. When data collected in routine care can be transferred to a FAIR-database of other hospitals or institutes, techniques of artificial intelligence (e.g., rapid learning) can be applied to use data of clinical practice in making more accurate predictions about treatment outcomes when monitoring levels of physical functioning throughout the perioperative period. Current technology is available in radiation oncology and at TRL level 4-6 [71, 72]. It is advocated that physical therapists align on their practices and cooperate with national data registries, such as the Dutch Institute for Clinical Auditing, and together should associate with the national healthdata infrastructure, such as Health-RI. Consequently, the combination of data and continuous comparative effectiveness might inform patients and their caregivers better on the type of treatments and outcomes. Second, the readiness for change and willingness to share data should be increased. Lustberg et al. [73] reported that the barriers for technological innovations and data-sharing are not of technological or ethical nature; however, barriers might be a lack of resources, expertise, and willingness to share data of health care professionals and researchers [73]. The absence of willingness to share data results from an ego-centered belief; the feeling of competition between institutes and not allowing comparison of performances with each other [73]. Therefore, it is recommended that professionals working in the field of perioperative care align on the readiness to invest in resources to build FAIR-databases, build-up expertise, and move from an ego-centered system towards an eco-centered system in which they take social responsibility to enhance data-management to improve patient outcomes. Only this way, SRL might come closer to TRL innovations.

Societal impact and citizen science: for the people, with the people, and by the people Science has become a more public activity, and aspects of science and its system are under discussion [5-8]. Governments and research institutes join (inter)national developments regarding science in transition and its increasing value, while simultaneously reducing waste in health research, which marks the importance of societal impact [74]. Societal impact is a popular term; however, a clear definition and a clear methodology to evaluate societal impact of research is still difficult [75]. Citizen science, defined as the cooperation of citizens as non-professional researchers in scientific research, has been recognized as a way to increase public support for 
research that might increase the change of scientific and societal impact of research [76]. Technological advances (availability of smartphones and wearables, high TRLs), but also the willingness by society increases the adoptions of innovations in SRLs [77]. The embedded science approach used in this thesis can also be seen as a way of citizen science. The embedded science approach led to a co-creation between patients, relatives, and health care professionals in the development of knowledge, which concurrently changed daily clinical practice based on daily interactions. However, the way citizens can contribute, collaborate or co-create with research and how citizens could have the lead in research should be further explored [78], which is necessary to increase SRLs.

\section{TAKE HOME MESSAGES}

This thesis, in line with the daily practice and evidence from the literature, illustrates the importance of a patient's physical functioning level before, during, and after hospitalization and surgery. The approaches presented here contribute to the ongoing and necessary transition of health care, where besides a medical-oriented reactive approach a more function-oriented proactive approach is implemented $[79,80]$, thereby focusing on a patient's vital functioning. The following clinical- and research-based take home messages can be put forward:

- $\quad$ nationally, a wide variation exists between reported pre- and postoperative clinical physical therapy management for patients opting for major abdominal surgery because of colorectal, hepatic, or pancreatic malignancies: alignment on further optimization of physical therapy management according to current evidence and advanced practices by some experts is advocated, like adequate preoperative risk stratification and prehabilitation (Chapter 2);

- $\quad$ an embedded science approach enables a researcher to integrate and perform research in daily clinical practice and to build academic partnerships between patients, health care professionals, and researchers for continuous (de)implementing, monitoring, and improving care innovations throughout the people's journey in a real-life context. This introduces a way to overcome the inequalities between the stages and moments of TRLs and SRLs of innovative tools and services and consequently adoption of these might become easier (Chapter 3);

- $\quad$ predictive perioperative physical therapy should become an integral part of the preoperative assessment, as a patient's preoperative level of physical functioning is associated with short-term postoperative outcomes in individual patients (Chapter 4 and Chapter 5);

- $\quad$ preventive perioperative physical therapy should be offered to individual patients classified at an increased risk for adverse postoperative outcomes and the program should be 
personalized and participatory, tailored to a patient's individual needs and social and physical context (Chapter 6);

- $\quad$ given the high rate of morbidity in patients following hepatic or pancreatic resection, the impact of any given postoperative event might be influenced by a patient's preoperative level of physical functioning (Chapter 6);

- $\quad$ physical therapists should explore the boundaries of their professions, in search for options for proper data management, as well as for options for citizen science to improve the content and context of perioperative physical therapy management and ultimately the outcomes of the patients involved (Chapter 7). 
1. Shapiro SD. Healthcare reform: it is getting personal. Per Med. 2012;9:405-12.

2. Shapiro SD. Update: healthcare reform it's getting personal. Per Med. 2017;14:281-3.

3. Hood L, Flores M. A personal view on systems medicine and the emergence of proactive P4 medicine: predictive, preventive, personalized and participatory. N Biotechnol. 2012;29:613-24.

4. Mulder I, Loorbach D. Rethinking design: a critical perspective to embrace societal challenges. in Proceedings of Transition Design Symposium, Devon, UK. 2016.

5. Chalmers I, Bracken MB, Djulbegovic B, Garattini S, Grant J, Gülmezoglu AM, et al. How to increase value and reduce waste when research priorities are set. Lancet. 2014 Jan 11;383:156-65.

6. Chan AW, Song F, Vickers A, Jefferson T, Dickersin K, Gøtzsche PC, et al. Increasing value and reducing waste: addressing inaccessible research. Lancet. 2014;383:257-66.

7. Dijstelbloem H, Huisman F, Miedema F, Mijnhardt, W. Waarom de wetenschap niet werkt zoals het moet en wat daar aan te doen is. in Position paper. 2013, Science in Transition. p. 31.

8. Moher D, Glasziou P, Chalmers I, Nasser M, Bossuyt PMM, Korevaar DA, et al. Increasing value and reducing waste in biomedical research: who's listening? Lancet. 2016;387:1573-86.

9. Mazzucato, M. Mission-oriented research \& innovation in the European Union. Brussels: European Commission. 2018.

10. DANDELION - Promoting EU - funded projects of inclusive, innovative and reflective societies (IIRS). 2016. Accessed may 2019,

http://www.dandelionEurope.eu/imagem/IIR S_sensitive_Valorisation_Concept.pdf.

11. European Commission. HORIZON 2020: Work Programme 2014-2015. 2014.

Accessed May 2019, http://ec.europa.eu/research/participants/d ata/ref/h2020/wp/2014_2015/main/h2020wp1415-health_en.pdf.
12. Ettorchi-Tardy A, Levif M, Michel P. Benchmarking: a method for continuous quality improvement in health. Healthc Policy. 2012;7:e101-19.

13. Luce BR, Kramer JM, Goodman SN, Connor JT, Tunis S, Whicher D, et al. Rethinking randomized clinical trials for comparative effectiveness research: the need for transformational change. Ann Intern Med. 2009;151:206-9.

14. Bongers BC, Punt IM, van Meeteren NL. On "Prehabilitation: The emperor's new clothes or a new arena for physical therapists?" Lundberg M, Archer KR, Larsson C, Rydwik E. Phys Ther. 2018;12:127-130. Phys Ther. 2019 ;99:953-4.

15. Filipe A, Renedo A, Marston C. The coproduction of what? Knowledge, values, and social relations in health care. PLoS Biol. 2017;15:e2001403

16. Vindrola-Padros $\mathrm{C}$, Eyre $\mathrm{L}$, Baxter $\mathrm{H}$, Cramer H, George B, Wye L, et al. Addressing the challenges of knowledge co-production in quality improvement: learning from the implementation of the researcher-in-residence model. BMJ Qual Saf. 2019;28:67-73.

17. Rawlins M. De Testimonio: on the evidence for decisions about the use of therapeutic interventions. Clin Med (Lond). 2008;8:579-88.

18. Sullivan R, Peppercorn J, Sikora K, Zalcberg J, Meropol NJ, Amir E, et al. Delivering affordable cancer care in highincome countries. Lancet Oncol. 2011;12:933-80

19. Conway PH, Clancy C. Comparativeeffectiveness research--implications of the Federal Coordinating Council's report. N Engl J Med. 2009;361:328-30.

20. Garber AM, Tunis SR. Does comparativeeffectiveness research threaten personalized medicine? N Engl J Med. 2009;360:1925-7. 
21. Raad van Volksgezondheid en

Samenleving. No evidence without context.

About the illusion of evidence-based practice in healthcare. 2017. Accessed June 11, 2019,

https://www.raadrvs.nl/documenten/public ations/2017/6/19/no-evidence-withoutcontext.-about-the-illusion-ofevidence\%E2\%80\%90based-practice-inhealthcare.

22. Lewens $T$. Distinguishing treatment from research: a functional approach. J Med Ethics. 2006;32:424-9.

23. Miller FG, Joffe $S$. Equipoise and the dilemma of randomized clinical trials. $\mathrm{N}$ Engl J Med. 2011;364:476-80.

24. Joffe S, Miller FG. Bench to bedside: mapping the moral terrain of clinical research. Hastings Cent Rep. 2008;38:3042.

25. Brown CJ, Redden DT, Flood KL, Allman RM. The underrecognized epidemic of low mobility during hospitalization of older adults. J Am Geriatr Soc. 2009;57:1660-5.

26. Koenders N, van Oorsouw R, Seeger JPH, Nijhuis-van der Sanden MWG, van de Glind I, Hoogeboom TJ. "I'm not going to walk, just for the sake of walking...": a qualitative, phenomenological study on physical activity during hospital stay. Disabil Rehabil. 2018;9:1-8.

27. Lutz W, Martinovich Z, Howard KI. Patient profiling: an application of random coefficient regression models to depicting the response of a patient to outpatient psychotherapy. J Consult Clin Psychol. 1999;67:571-7.

28. Hofstraat $H$, Joachimsthal $M$, Lagendijk I, Mons B, Vink H, Kok R, et al. Vitaal functionerende burgers in een gezonde economie met support vanuit big 'complex' data. 2019:1-12.

29. Greenblatt DY, Kelly KJ, Rajamanickam V, Wan Y, Hanson T, Rettammel R, et al. Preoperative factors predict perioperative morbidity and mortality after pancreaticoduodenectomy. Ann Surg Oncol. 2011;18:2126-35.
30. Breitenstein S, DeOliveira ML, Raptis DA, Slankamenac K, Kambakamba P, Nerl J, et al. Novel and simple preoperative score predicting complications after liver resection in noncirrhotic patients. Ann Surg. 2010;252:726-34.

31. Yu DC, Chen WB, Jiang CP, Ding YT. Risk assessment in patients undergoing liver resection. Hepatobiliary Pancreat Dis Int. 2013;12:473-9.

32. Levett DZH, Jack S, Swart M, Carlisle J, Wilson J, Snowden C, et al. Perioperative Exercise Testing and Training Society (POETTS). Perioperative cardiopulmonary exercise testing (CPET): consensus clinical guidelines on indications, organization, conduct, and physiological interpretation. Br J Anaesth. 2018;120:484-500.

33. Moran J, Wilson F, Guinan E, McCormick P, Hussey J, Moriarty J. Role of cardiopulmonary exercise testing as a riskassessment method in patients undergoing intra-abdominal surgery: a systematic review. Br J Anaesth. 2016;116:177-91.

34. Junejo MA, Mason JM, Sheen AJ, Bryan A, Moore J, Foster P, et al. Cardiopulmonary exercise testing for preoperative risk assessment before pancreaticoduodenectomy for cancer. Ann Surg Oncol. 2014;21:1929-36.

35. Junejo MA, Mason JM, Sheen AJ, Moore J, Foster P, Atkinson D, et al.

Cardiopulmonary exercise testing for preoperative risk assessment before hepatic resection. $\mathrm{Br} \mathrm{J}$ Surg. 2012;99:1097-104.

36. Dronkers JJ, Chorus AM, van Meeteren NL, Hopman-Rock M. The association of pre-operative physical fitness and physical activity with outcome after scheduled major abdominal surgery. Anaesthesia. 2013;68:67-73.

37. Heldens AFJM, Bongers BC, Lenssen AF, Stassen LPS, Buhre WF, van Meeteren NLU. The association between performance parameters of physical fitness and postoperative outcomes in patients undergoing colorectal surgery: An evaluation of care data. Eur J Surg Oncol. 2017;43:2084-92. 
38. Hayashi K, Yokoyama Y, Nakajima H, Nagino $M$, Inoue T, Nagaya $M$ et al.Preoperative 6-minute walk distance accurately predicts postoperative complications after operations for hepatopancreato-biliary cancer. Surgery. 2017; 161:525-32.

39. Vickers AJ. Prediction models: revolutionary in principle, but do they do more good than harm? J Clin Oncol. 2011;29:2951-2.

40. Thomas G, Tahir MR, Bongers BC, Kallen VL, Slooter GD, van Meeteren NL. A systematic review of randomised controlled trials investigating prehabilitation before major intra-abdominal cancer surgery: an analysis of prehabilitation content and outcome measures. Eur J Anaesthesiol. In press.

41. Steyerberg EW. Statistical models for prediction. In: Steyerberg EW, editor. Clinical Prediction Models:A Practical Approach to Development, Validation, and Updating. 1st ed. New York: SpringerVerlag; 2009:53-83.

42. Cardoso AL, Fernandes A, AguilarPimentel JA, de Angelis MH, Guedes JR, Brito MA et al. Towards frailty biomarkers: Candidates from genes and pathways regulated in aging and age-related diseases. Ageing Res Rev. 2018;47:214277.

43. Buettner S, Wagner D, Kim Y, Margonis GA, Makary MA, Wilson A et al. Inclusion of Sarcopenia Outperforms the Modified Frailty Index in Predicting 1-Year Mortality among 1,326 Patients undergoing Gastrointestinal Surgery for a Malignant Indication. J Am Coll Surg. 2016;222:397407.

44. Wijeysundera DN, Pearse RM, Shulman MA, Abbott TEF, Torres E, Ambosta A et al.METS study investigators. Assessment of functional capacity before major noncardiac surgery: an international, prospective cohort study. Lancet. 2018;391:2631-2640.

45. Barberan-Garcia A, Ubré M, Roca J, Lacy AM, Burgos F, Risco R et al. Personalised Prehabilitation in High-risk Patients Undergoing Elective Major Abdominal Surgery: A Randomized Blinded Controlled Trial. Ann Surg. 2018 Jan;267(1):50-56.
46. Bruns ER, van den Heuvel B, Buskens CJ, van Duijvendijk P, Festen S, Wassenaar $E B$, et al. The effects of physical prehabilitation in elderly patients undergoing colorectal surgery: a systematic review. Colorectal Dis. 2016;18:O267-77.

47. Moran J, Guinan E, McCormick P, Larkin J, Mockler D, Hussey J, et al. The ability of prehabilitation to influence postoperative outcome after intra-abdominal operation: A systematic review and meta-analysis. Surgery. 2016;160:1189-1201.

48. Hoogeboom TJ, Oosting E, Vriezekolk JE, Veenhof C, Siemonsma PC, de Bie RA, et al. Therapeutic validity and effectiveness of preoperative exercise on functional recovery after joint replacement: a systematic review and meta-analysis. PLoS One. 2012;7:e38031

49. Dronkers J, Witteman B, van Meeteren N. Surgery and functional mobility: doing the right thing at the right time. Tech Coloproctol. 2016;20:339-341.

50. Berkel AEM, Bongers BC, van Kamp MS, Kotte $\mathrm{H}$, Weltevreden $\mathrm{P}$, de Jongh FHC, et al. The effects of prehabilitation versus usual care to reduce postoperative complications in high-risk patients with colorectal cancer or dysplasia scheduled for elective colorectal resection: study protocol of a randomized controlled trial. BMC Gastroenterol. 2018;18:29.

51. Hoogeboom TJ, Dronkers JJ, van den Ende $\mathrm{CH}$, et al. Preoperative therapeutic exercise in frail elderly scheduled for total hip replacement: a randomized pilot trial. Clin Rehabil. 2010;24:901-10.

52. Oosting E, Jans MP, Dronkers JJ, Naber $\mathrm{RH}$, Dronkers-Landman CM, Appelman-de Vries SM, et al Preoperative home-based physical therapy versus usual care to improve functional health of frail older adults scheduled for elective total hip arthroplasty: a pilot randomized controlled trial. Arch Phys Med Rehabil. 2012;93:6106.

53. Schmidt, R.A. and T.D. Lee, Motor learning and performance: from principles to application. 2018: Human Kinetics.

54. Hulzebos EH, van Meeteren NL. Making the elderly fit for surgery. $\mathrm{Br} \mathrm{J}$ Surg.2016;103:463. 
55. Hulzebos EH, van Meeteren NL. Making the elderly fit for surgery. Br J Surg. 2016;103:e12-5.

56. Hulzebos EH, Helders PJ, Favié NJ, De Bie RA, Brutel de la Riviere A, Van Meeteren NL. Preoperative intensive inspiratory muscle training to prevent postoperative pulmonary complications in high-risk patients undergoing CABG surgery: a randomized clinical trial. JAMA. 2006;296:1851-7.

57. Covinsky KE, Pierluissi E, Johnston CB. Hospitalization-associated disability: "She was probably able to ambulate, but I'm not sure". JAMA. 2011;306:1782-93.

58. Burdelski CM, Reeh M, Bogoevski D, Gebauer F, Tachezy M, Vashist YK, et al. Multivisceral resections in pancreatic cancer: identification of risk factors. World J Surg. 2011;35:2756-63.

59. Van Genderen FR, De Bie RA, Helders PJ, Van Meeteren NL. Reliability research: towards a more clinically relevant approach. Physical therapy reviews. 2003;8:169-76.

60. Lillie EO, Patay B, Diamant J, Issell B, Topol EJ, Schork NJ. The n-of-1 clinical trial: the ultimate strategy for individualizing medicine? Per Med. 2011;8:161-173.

61. Peterson A. Improving type 1 diabetes management with mobile tools: a systematic review. J Diabetes Sci Technol. 2014;8:859-64.

62. Glasziou P, Irwig L, Mant D. Monitoring in chronic disease: a rational approach. BMJ. 2005;330:644-8.

63. Hoogeboom TJ, Kittelson AJ, van der Sluis $\mathrm{G}$, van Meeteren NL. Evidence-based practice in physiotherapy: time to involve the patient? Physiotherapy. 2014;100:3634.

64. Scheede-Bergdahl C, Minnella EM, Carli F. Multi-modal prehabilitation:addressing the why, when, what, how, who and where next? Anaesthesia. 2019;74 Suppl 1:2026.

65. Grocott MPW, Edwards M, Mythen MG, Aronson S. Peri-operative care pathways: re-engineering care to achieve the 'triple aim'. Anaesthesia. 2019;74 Suppl 1:90-99.
66. Oosting E, Dronkers J, Hoogeboom T, van Meeteren N, Speelman WM. Personal meaning in relation to daily functioning of a patient in physical therapy practice: narratives of a patient, a family member, and physical therapist. Disabil Rehabil. 2018;40:1220-6.

67. Lopopolo RB. Hospital restructuring and the changing nature of the physical therapist's role. Phys Ther. 1999;79:17185.

68. Alharthi A, Krotov V, Bowman M. Addressing barriers to big data. Business Horizons. 2017;60:285-92.

69. Bellazzi R. Big data and biomedical informatics: a challenging opportunity. Yearb Med Inform. 2014;9:8-13.

70. Wilkinson MD, Dumontier M, Aalbersberg IJ, Appleton G, Axton M, Baak A, et al. The FAIR Guiding Principles for scientific data management and stewardship. Sci Data. 2016;3:160018.

71. Lambin P, Roelofs E, Reymen B, Velazquez ER, Buijsen J, Zegers CM, et al. 'Rapid Learning health care in oncology' - an approach towards decision support systems enabling customised radiotherapy'. Radiother Oncol. 2013;109:159-64.

72. Lambin P, Zindler J, Vanneste BG, De Voorde LV, Eekers D, Compter I, et al. Decision support systems for personalized and participative radiation oncology. Adv Drug Deliv Rev. 2017;109:131-153.

73. Lustberg T, van Soest J, Jochems A, Deist $T$, van Wijk $Y$, Walsh S, et al. A. Big Data in radiation therapy: challenges and opportunities. Br J Radiol. 2017;90:20160689.

74. Van der Meulen B, Maclaine Pont $P$, Faasse P, Deuten J, Belder R. Keuzes voor de toekomst van de nederlandse wetenschap. 2015. Accessed June, 2019. https://www.rathenau.nl/nl/vitalekennisecosystemen/keuzes-voor-detoekomst-van-de-nederlandsewetenschap.

75. Bornmann L. Measuring the societal impact of research: research is less and less assessed on scientific impact alone-we should aim to quantify the increasingly important contributions of science to society. EMBO Rep. 2012;13:673-6. 
76. Den Broeder L, Devilee J, Van Oers H, Schuit AJ, Wagemakers A. Citizen Science for public health. Health Promot Int. 2018;33:505-14.

77. Bonney R, Shirk JL, Phillips TB, Wiggins A, Ballard HL, Miller-Rushing AJ, et al. Citizen science. Next steps for citizen science. Science. 2014;343:1436-7.

78. Reijmerink W. De impact van publieke kennisprogrammering. Tijdschrift voor gezondheidswetenschappen. 2018;96:1244-7.
79. Health-Holland, Knowledge and Innovation Agenda 2020-2023, Top Sector Life Sciences and Health. Schuttelaar \& Partners Den Haag, 2019.

80. Ministerie van Economische Zaken en Klimaat. Missies voor het topsectoren- en innovatiebeleid - 26 april 2019. Accessed June 11, 2019, https://www.topsectoren.nl/missiesvoordet oekomst/documenten/kamerstukken/2019/ april/29-04-2019/missiedocument.

\section{NOTES}


Regionale netwerkvorming in 'learning communities' is een uitdaging, maar een noodzakelijke voorwaarde om de juiste zorg, op de juiste plek, op het juiste moment te leveren. 
Chapter 8

Valorization 

Today's national societal challenges - ranging from the energy transition and sustainability, to health care, agriculture, water, and food - in combination with innovative technologies - like digitalization - will both radically change society and will influence economic activities. Considering health care challenges, innovation is the key driver to move from a reactive approach towards a predictive, preventive, personalized, and participatory approach focusing on vital functioning citizens [1]. Innovation requires proper research to develop new knowledge, ideas, and products and bringing them from bench to bedside, to the community and back. However, a significant gap exists between developed knowledge in research practice and the actual improvement in health care practice [2].

Dutch universities have the legal task - besides education and conducting research - to overcome this gap and transfer knowledge for the benefit of society (Higher Education and Research Act [WHW], 1192, article 1.3). In 2004, this task was labeled by the Dutch Minister of Science Van der Hoeven under the term 'valorization' [3]. Valorization means 'the process of creating value from knowledge by making it suitable and/or available for economic and/or societal use and translating it into competitive products, services, processes, and entrepreneurial activity' [4]. However, the performance of valorization and many aspects of science are under discussion. The Dutch Science in Transition movement [5] prefers to speak of valorization in terms of societal impact, in which academics take more societal responsibility and not only produce knowledge in terms of scientific publications. Internationally, the European Commission published a document regarding 'Science in transition 2.0', discussing the necessary steps to change the dynamics of research to address societal challenges [6]. Furthermore, the Lancet published five papers regarding how to decrease research waste and improve research quality [7-11]. So, science performed in universities is expected not only to develop and transfer knowledge, but should also address societal challenges and contribute to societal benefits.

One specific challenge is the maintenance of people's vital functioning before, during, and after a major life event, such as surgery. High-risk patients opting for major surgery experience a decrease in their physical functioning due to their hospitalization and surgery. Some patients already experience a decrease in their physical functioning before surgery, due to their specific illness and/or due to the passive "sit, wait, and see" approach in the preoperative period by the patients themselves, their family, or by informal caregivers. After surgery, patients with a low level of physical functioning have an increased risk for postoperative complications, require extensive postoperative rehabilitation, and are more likely to have a re-admission [12]. 
This chapter reflects on the social and economic relevance of an at the niche level performed mission-oriented research program as described in this thesis. The research program involved a transition in perioperative physical therapy care, with the aim to improve a patient's physical functioning during a major life event episode, especially in patients undergoing elective hepatic or pancreatic resection. Additionally, it will elaborate on how this at the niche level performed mission-oriented research can contribute to the defined Dutch health care mission, which states that "by 2040, all Dutch citizens will live at least five years longer in good health, while health inequalities between the lowest and highest socio-economic groups will have decreased by $30 \%$ " [1] using the multi-level perspective framework.

\section{SOCIAL AND ECONOMIC RELEVANCE OF A TRANSITION IN PERIOPERATIVE CARE}

In order to be able to participate in society and to remain independent, optimal physical functioning is important. Being able to perform activities of daily life on a physical, mental, and social level increases quality of life and ensures the ability to engage in society. Besides the agerelated loss of physical functioning, patients opting for surgery might experience a decrease in their level of physical functioning, especially those patients who are already vulnerable. Surgery for cancer in the hepato-pancreato-biliary tract involves complex procedures. Patients should be able to cope with the physiological stress response that coincides with hospitalization and surgery. When a patient is unable to adapt adequately to this physiological stress response, patients have a higher risk for a delayed postoperative recovery of physical functioning or even permanent loss of physical functioning.

In both the pre- and postoperative phase, the physical therapist - in co-creation with the patient, family, and other (in)formal caregivers - should support a patient opting for major surgery to achieve and maintain their own control (patient empowerment) regarding their level of physical functioning in order to prevent deterioration. Some physical therapy departments in Dutch hospitals have taken up this responsibility and have adopted pre- and postoperative strategies to maintain and improve a patient's physical functioning before and after hospitalization and surgery. However, there is still a large variation and gap between the current clinical perioperative physical therapy practice and the advised state-of-the-art evidence [13].

By knowing this gap between the evidence in the literature and clinical practice, an embedded science approach is a way to reduce the gap. An embedded scientist combines working in daily clinical practice with scientific evaluations of the delivered care by continuously monitoring, 
improving, and evaluating health care interventions throughout the patient journey in the real-life context. At the niche level at the Maastricht University Medical Center+, the embedded science approach resulted in changes in daily clinical practice (e.g., an outpatient preoperative assessment unit to assess a patient's physical fitness and physical functioning, a communitybased exercise prehabilitation network, and postoperative monitoring of a patient's physical functioning). Equally, it led to alterations in patient outcomes after elective hepatic and pancreatic resection (e.g., a reduced median time to recovery of physical functioning and a reduced length of hospital stay) [14]. Thereby, this approach created public value by validating and integrating so called 'point' solutions in the real-life context of patients, leading to better patient outcomes in terms of physical functioning.

At the same time, the embedded science approach enables to create new theories and perspectives about perioperative physical therapy management at a micro (department of physical therapy), macro (interdisciplinary teams), and meso (regional prehabilitation network or national) level. This at the niche level performed mission-oriented research program resulted in adaptive academic partnerships between patients, health care professionals, and researchers within the Maastricht University Medical Center+ (micro and macro level), and led to coproduction in exploring new forms of knowledge, values, and social relations.

An additional argument for optimizing perioperative care might be the potential return of investment in preventive perioperative physical therapy interventions. For example, median costs of an uncomplicated pancreatoduodenectomy are $€ 17.500$; however, these might increase by $34 \%$ to $71 \%$ when a complication occurs [15]. One should be aware that the average annual investment in an embedded scientist is $€ 51.118$, and the median investment in prehabilitation for a single patient is $€ 320$ (8 sessions of 30 minutes, $€ 40$ for each session), leading to a $25 \%$ improvement of a patient's level of physical functioning, as shown in a single case (Chapter 6$)$. In the four years of this mission-oriented research program, a reduction in median hospital length of stay (4 days in pancreatic resection and 1 day in hepatic resection) and a reduction in median time to recovery of physical functioning ( 3 days in pancreatic resection and 1 day in hepatic resection) was observed, which might be attributed to changes in health care practices. In a recent cost-consequence analysis of Barberan-Garcia et al., [16] prehabilitation in high-risk patients undergoing major abdominal surgery reduced postoperative complications (relative risk of $0.5 ; 95 \%$ confidence interval ranging from 0.3 to 0.8 ) without increasing direct health care costs; hence, prehabilitation seems a cost-effective preventive preoperative intervention when 
performed in high-risk patients. These economic considerations might be an additional argument to take a closer look into the business case of optimizing perioperative care.

\section{PRACTICAL INNOVATIONS}

The initiation of the transition towards a proactive perioperative physical therapy care pathway resulted in several (sustainable) changes at the Maastricht University Medical Center+ related to the preoperative phase, postoperative phase, and collaboration in learning communities.

\section{Preoperative physical therapy}

Since 2016, patients who are diagnosed with cancer in the liver or pancreas and opt for elective abdominal surgery can participate in a preoperative screening of their level of physical functioning and physical fitness as part of usual care. During this screening, several practical performancebased tests are performed, where after patients receive information regarding the results and an advice on how to prepare themselves for surgery. Data from the preoperative screening were used in the cohort studies (Chapter 4 and Chapter 5). In these studies, the conventional patientrelated medical (American Score of Anesthesiologist score) and demographic (body mass index) were associated with the time to recovery of physical functioning. Besides these conventional patient-related medical and demographic risk factors, people with a lower aerobic capacity, a worse functional mobility, and a lower perceived level of functional capacity to perform activities of daily life were more likely to experience a delayed recovery of physical functioning after surgery. These results emphasize the importance of an adequate level of physical functioning before major surgery but also emphasize the importance of a proactive role of the physical therapist. Nowadays, this knowledge is used as part of usual care to inform patients regarding the importance of physical activity and physical fitness. Patients with an increased risk for a delayed recovery of physical functioning have the opportunity to participate in a home-based functional exercise training program.

\section{Postoperative physical therapy}

Nowadays, daily monitoring of a patient's recovery of physical functioning is an integral part in the postoperative physical therapy care for patients who underwent hepatic or pancreatic resection. The modified lowa level of assistance scale ( $\mathrm{mILAS})$ is used to monitor five functional tasks related to physical functioning. Furthermore, also other practical performance-based-tests of relevance for the patient (e.g., five times sit-to-stand test, two-minute walk test) are used to monitor recovery of physical functioning. Based on the test results, frequency and intensity of physical therapy is 
adapted. In addition, a poster was designed to create more awareness regarding the importance of physical activity during hospitalization. This poster contains activities of daily living (e.g., bathing, eating, walking, climbing stairs) and the level of (in)dependence performing these activities.

\section{Transfer of knowledge in learning communities}

The knowledge developed within this thesis is due to collaborations between patients, caregivers, and scientists, who share the same common goals to improve perioperative care (e.g., participated in a learning community). Within the catchment area of the Maastricht University Medical Center+ (30 km), a learning community was established. Within this learning-community, there was a co-creation between the 'tacit' knowledge of practitioners in the field in combination with scientific and process knowledge of researchers. This led to a co-evolving theory and improved patient outcomes, which was due to a change in daily clinical practice in the perioperative management of patients that decided to undergo major elective abdominal surgery because of hepatic or pancreatic cancer. As part of this learning community, a prehabilitation network with trained community physical therapists was developed to support and train high-risks patients in their own living context. Each community physical therapist committed to the network followed a three-day course and a decision-support guideline was developed regarding the homebased functional task training with a small group of physical therapists from the prehabilitation network. Nationally, a Dutch learning community of practice, consisting of clinicians, embedded scientists, and policy makers working in the field of perioperative physical therapy care, shared practical experiences and research insights, thereby aiming to improve perioperative care with patients opting for major surgery. By doing so, it is not only about maintaining and improving a patient's physical functioning throughout the people's journey, but also about remaining critical towards the content and context of health care procedures.

\section{FROM NICHE-LEVEL MISSION-ORIENTED INITIATIVES TOWARDS LANDSCAPE MISSIONS}

The European Union is shifting towards a mission-oriented research approach to address global societal challenges [17]. Within this approach, a clear mission is defined and collaborations between public and private organizations, local communities, non-governmental organizations, and other societal actors are set-up to achieve societal impact and pursuit economic growth [10]. The Dutch Ministry of Economic Affairs and Climate Policy endeavors this mission-driven innovation policy with collaboration within the quadruple helix (citizens, governments, entrepreneurs, and researchers) to address societal challenges [18]. The central mission for the 
Dutch health care transition is as follows: "In 2040, all Dutch people will live in good health for at least five years longer, and the health differences between the lowest and highest socioeconomic groups will have decreased by 30\%" [1]. The mission-oriented approach to system innovation is not a top-down approach, but rather creating an environment of a living-lab in which experimentation, policy learning, and stakeholder participation is encouraged [10]. The question remains how to create such an environment that bottom-up initiatives - like the niche missionoriented research in perioperative physical therapy care - will not stagger. By making use of the multilevel perspective framework, this thesis aimed to provide an overview of the actions that could be undertaken to drive the optimization of perioperative care at niche level.

The multi-level perspective in the perioperative care transition

The multilevel perspective describes a societal system at three levels (micro, meso, and macro), in which system-innovations (transition experiments) interplay between different levels and in different phases (see Figure 8.1) [19, 20]. Bottom-up experiments at the micro-level are called niches. These niches provide space for learning processes in which different actors find out if novelties based on scientific research are value-adding to the patient and will improve perioperative care. The meso-level is called the socio-technical regime, and accounts for the stability in the existing routine perioperative care setting. Regimes encompass technologies, institutions, and actors who change incrementally. The macro-level, also called the sociotechnical landscape, cannot change in the short-term, and is beyond direct influence of the actors in the transition experiments. However, it can put pressure on the existing regime and/or opens a window of opportunity for niche experiments.

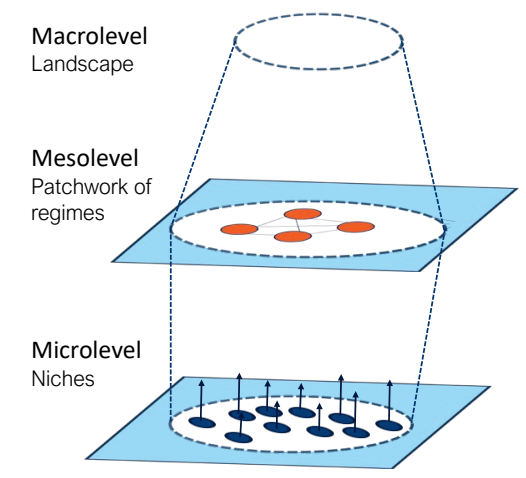

Figure 8.1. The different scale levels of a transition (modified from Geels [20]). Bottom-up niches, the top-down landscape, and regimes within perioperative health care to improve physical functioning throughout the people's journey are depicted. 


\section{Niche}

A niche can be seen as a group of actors who co-create in order to develop deviating innovative social, economic, and technological practices, and are protected from the dominant regime [19]. In 2018, various regional and thematic niches were initiated in the Netherlands in order to maintain and increase the rate of innovations. These regional and thematic niches are called learning communities [21]. In these learning communities or so-called 'living labs', the quadruple helix joins forces to explore - with support of technological innovations - solutions for a specific challenge in a specific context $[22,23]$. The local niche experiment in perioperative physical therapy care as described above - already functioning as a learning community - might consider exploring further collaboration with regional level learning communities. For instance, by learning from the living lab of healthy ageing and long-term care. In the latter mentioned living lab, interdisciplinary collaboration among citizens, embedded innovators, policy makers, and educators exists, and they are successful in creating an innovative care environment [24]. By broadening the learning community of perioperative care with more technology experts, technological innovations for maintaining a patient's pre- and postoperative level of physical functioning can be intertwined with socio-cultural innovations at the same time, in order to achieve sustainable implementations of innovations in the life of patients and/or the professional. Furthermore, niches can also make use of the Dutch Top Sector public-private-partnerships, in which multi-stakeholders in a specific field of expertise work together (intellectual, human, and financial capital) to perform science, but also to work on outcomes and deliverables with both social and economic impact [25]. For perioperative care, such a regional public-privatepartnership has been set-up with patients, hospitals, universities, and national data registries to create a clinimetric composite toolbox for better predicting surgical outcomes in patients who undergo elective colorectal surgery [26]. The above-mentioned considerations are based on the idea that niche development should incorporate the quadruple helix in order to create a sustainable regional knowledge and innovation structure, in which local, regional (province), and national governments should help by investing in these infrastructures.

\section{Regime}

The regime is the dominant culture, structure, and practice in a system. Applying the theory of Geels [27], regime elements in perioperative care can be tangible (e.g., laws, guidelines, protocols, standard practices) or intangible (e.g., policy paradigms, organization structure, norms and beliefs). Important key actors in the regime and their associated institutional structures (e.g., hospitals, universities, health insurance companies, expertise networks) influence the regime 
elements, and thereby determine the culture, structure, and practice in a system. Changes in the regime might develop when the regime is stressed by tension, which influences daily practice. For instance, surgical care is stressed by the ageing population [28]. Linking and introducing new initiatives in daily perioperative care that might solve these tensions might trigger readiness for change in the regime. Furthermore, the development of (international) guidelines regarding changes in perioperative care [29] creates more tangible elements to change the regime, but also influences norms and beliefs of health care professionals, which might influence their practices.

Another way to influence the regime lies within the commitment of key actors. Key actors in the regime with policy power in institutions might directly employ activities or new structures within their organizations to improve and expand new elements in the regime [20]. For instance, policy makers within universities or hospitals might reconsider their organizational structure to better adapt to the evolving environment and new technologies to improve their performance in terms of social and economic impact. Institutes might consider to change from a hierarchical silo structure towards a dynamic agile organization (see Figure 8.2) [30]. Key-actors working in a dynamic agile organization should aim to create an ecosystem with a dense network of empowered teams that enable them to learn and experiment beyond the boundaries of the regime. By doing so, policy makers can create cross-functional teams, which are also called dynamic capabilities of a company [31]. A dynamic capability is the ability to integrate (put resources together), build, and reconfigure internal and external competencies to rapidly adapt to the changing environments [32]. A cross-functional team can be seen as a learning community with diverse actors in the organization, in which people interact and work on a clear mission while being supported by cross-team performance management. So, policy makers for perioperative care should connect knowledge, technology, and finances between institutes, as well as create diverse teams by not acting from an ego-centered belief, but from an eco-centered belief. This new way of working and thinking will also tie in with the 'science in transition' initiative [5]. 
From organizations as "machines"
... to organizations as

"organisms"
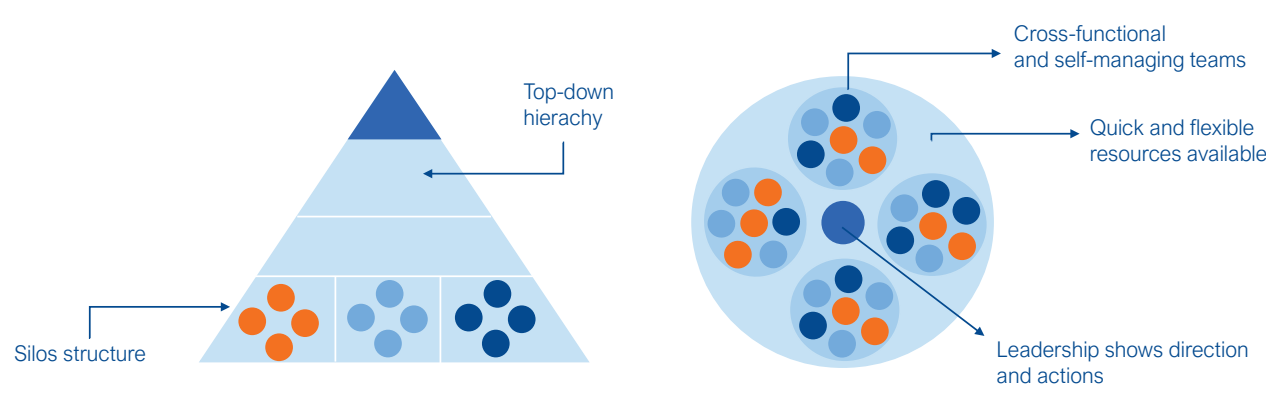

Figure 8.2. The change of the organization structure from a hierarchical silo structure with top-down leadership, towards a more 'dynamic' organization with cross-functional teams that are more able to act upon the changing environment (adapted from McKinesy \& Company [30]).

\section{Landscape}

The landscape is beyond the influence of niche and regime actors, and contains political, economic, and social changes, which will take more time (decades) [27]. The landscape can put pressure on the existing regime so that potential niches have the ability to become incorporated in the regime. For instance, local institutions can reconsider their policy regarding assessment methods of performance and reward systems. Simply counting the number of publications or performing a cost-benefit analysis cannot capture the potential social and economic impact of a certain project, team, or person. On a national level, departments of ministries developed political agendas or programs, like the 'national prevention act' and 'De Juiste Zorg op de Juiste Plek', to optimize the contribution of public-private-partnerships in the mission-driven innovation policy of the Top Sector [1].

\section{CONCLUSION AND AMBITIONS}

The ability to perform activities of daily live independently throughout the complete perioperative period is not obvious. However, when using an embedded science approach, a patient's physical functioning throughout the perioperative period can be improved by integrating, evaluating, and ecologically validating pre- and postoperative interventions. To better support citizens in their physical functioning during a major life event, the quadruple helix should combine forces and establish itself deeper in learning communities to create innovations that lead to societal and economic value. 


\section{REFERENCES}

1. Health-Holland. Knowledge and Innovation Agenda 2020-2023. Top Sector Life Sciences and Health. Schuttelaar \& Partners Den Haag, 2019.

2. Rangachari P, Rissing P, Rethemeyer K. Awareness of evidence-based practices alone does not translate to implementation: insights from implementation research. Qual Manag Health Care. 2013;22:117-25.

3. Ministry of Education, Culture and Science. (2004). Focus op excellentie en meer waarde: Wetenschapsbudget. Den Haag. 2004.

4. Nederland Ondernemend Innovatieland. Van voornemens naar voorsprong: kennis moet circuleren. Den Haag: Interdepartmentale Programmadirectie Kennis en Innovatie. 2009.

5. Dijstelbloem H, Huisman F, Miedema F, Mijnhardt, W. Waarom de wetenschap niet werkt zoals het moet en wat daar aan te doen is. Position paper. 2013, Science in Transition. p. 31.

6. European Union Commission. Validation of the results of the public consultation on Science 2.0: Science in Transition. Retrieved July 10, 2015.

7. Chalmers I, Bracken MB, Djulbegovic B, Garattini S, Grant J, Gülmezoglu AM, et al. How to increase value and reduce waste when research priorities are set. Lancet. 2014;383:156-65.

8. Chan AW, Song F, Vickers A, Jefferson T, Dickersin K, Gøtzsche PC, et al. Increasing value and reducing waste: addressing inaccessible research. Lancet. 2014;383:257-66.

9. Ioannidis JP, Greenland S, Hiatky MA, Khoury MJ, Macleod MR, Moher D, et al. Increasing value and reducing waste in research design, conduct, and analysis. Lancet. 2014;383:166-75.

10. Al-Shahi Salman R, Beller E, Kagan J, Hemminki E, Phillips RS, Savulescu J, et al. Increasing value and reducing waste in biomedical research regulation and management. Lancet. 2014;383:176-85.
11. Glasziou P, Altman DG, Bossuyt $P$, Boutron I, Clarke M, Julious S, et al. Reducing waste from incomplete or unusable reports of biomedical research. Lancet. 2014;383:267-76.

12. Dalton A, Zafirova Z. Preoperative management of the geriatric patient: frailty and cognitive impairment assessment. Anesthesiol Clin. 2018:36:599-614.

13. van Beijsterveld CA, Heldens AF, Bongers $B C$, van Meeteren NL. Variation in preoperative and postoperative physical therapist management of patients opting for elective abdominal surgery. Phys Ther. 2019;99:1291-303.

14. Van Beijsterveld CA, Bongers BC, Den Dulk M, Van Kuijk SMJ, Dejong CH, Van Meeteren NL. The association between preoperative physical functioning and short-term postoperative outcomes: a cohort study of patients undergoing elective hepatic resection. HPB (Oxford). 2019;21:1362-70.

15. Santema TB, Visser A, Busch OR, Dijkgraaf MG, Goslings JC, Gouma DJ, et al. Hospital costs of complications after a pancreatoduodenectomy. HPB (Oxford) 2015;17:723-31.

16. Barberan-Garcia A, Ubre M, PascualArgente N, Risco R, Faner J, Balust J et al. Post-discharge impact and costconsequence analysis of prehabilitation in high-risk patients undergoing major abdominal surgery: secondary results from a randomised controlled trial. $\mathrm{Br} \mathrm{J}$ Anaesth. 2019;123:450-6.

17. Mazzucato M. Mission-oriented research \& innovation in the European Union.

Brussels: European Commission. 2018.

18. Wiebes E, Keijzer M. Naar Missiegedreven Innovatiebeleid met Impact [Letter of government]. 2018.

19. Geels FW, Kemp R. Dynamics in sociotechnical systems: Typology of change processes and contrasting case studies. Technology in Society. 2007;29:441-55. 
20. Geels FW. Technological transitions as evolutionary reconfiguration processes: a multi-level perspective and a case-study. Research Policy. 2002:31:1257-74.

21. Human Capital Roadmap 2016-2020. Advies rapport Learning communities 2018-2022. 2017. Accessed October 2019, https://www.topsectoren.nl/humancapital.

22. Swinkels ICS, Huygens MWJ, Schoenmakers TM, Oude NijewemeD'Hollosy W, van Velsen L, Vermeulen J, et al. Lessons learned from a living lab on the broad adoption of eHealth in primary health care. J Med Internet Res. 2018;29;20:e83.

23. Schuurman D, Baccarne B, De Marez L, Veeckman C, Ballon P. Living Labs as open innovation systems for knowledge exchange: solutions for sustainable innovation development. International Journal of Business Innovation and Research. 2016;10:322-40.

24. Verbeek H, Zwakhalen SM, Schols JM, Hamers JP. Keys to successfully embedding scientific research in nursing homes: a win-win perspective. J Am Med Dir Assoc. 2013;14:855-7.

25. Groen HL, van Meeteren NL. The Value of Public-Private Partnerships in the Netherlands. Handbook of biomarkers and precision medicine. 2019:335.

26. Public-Private-Partnership: Relation between preoperative performance and postoperative outcome in colorectal cancer. Accessed October, 2019, https://www.healthholland.com/project/2017/relationbetween-preoperative-performance-testsand-postoperative-outcome-in-colorectalcancer.
27. Geels FW. The multi-level perspective on sustainability transitions: responses to seven criticisms. Environmental Innovation and Societal Transitions. 2011;1:24-40.

28. Søreide K, Wijnhoven BP. Surgery for an ageing population. Br J Surg. 2016;103:e7-9.

29. Tew GA, Ayyash R, Durrand J, Danjoux GR. Clinical guideline and recommendations on pre-operative exercise training in patients awaiting major non-cardiac surgery. Anaesthesia. 2018;73:750-68.

30. Aghina W, Ahlback K, De Smet A, Lackey G, Lurie M, Murarka M, et al. The five trademarks of agile organizations| McKinsey \& Company. January report 2018.

31. Miedzinski M, Mazzucato M, Ekins P. A Framework for Mission-Oriented Innovation Policy Roadmapping for the SDGs. UCL Institute for Innovation and Public Purpose, 2019.

32. Teece DJ, Pisano G, Shuen A. Dynamic capabilities and strategic management. Strategic management journal. 1997;18:509-33.

\section{NOTES}


"In der Beschränkung zeigt sich erst der Meister".

- J.W. von Goethe's citaat (1802) - 
Summary 



\section{SUMMARY}

Along with the rapid demographical changes, technological and sociocultural innovations drive changes in the health care sector. Currently, health care is in transition from a more or less reactive medical approach (cure and care), towards a proactive predictive, preventive, personalized, and participatory approach (P4 health) that focuses on people's daily functioning. To guide the transition, a grand mission-driven approach was initiated in the Netherlands from 2016 on, in which the mission for health care states: "In 2040, all Dutch people will live in good health for at least five years longer, and the health differences between the lowest and highest socioeconomic groups will have decreased by 30\%". Such a mission requires changes in the current health care practices and creates windows of opportunity for experimenting and validating intertwined technological and social innovations with patients and their relatives, health care professionals, scientists, and entrepreneurs.

\section{Our mission}

The aim of this mission-oriented thesis was to initiate a transition in perioperative physical therapy care from a reactive approach, towards proactive P4 perioperative physical therapy care to maintain vital functioning during major life events, specifically focusing on patients undergoing elective hepatic or pancreatic resection.

\section{Vital functioning before and after surgery}

Surgery for patients with cancer in the liver and pancreas is the primary modality of curative treatment. Maintaining vital physical functioning during a major life event such as surgery and the accompanied hospitalization is challenging. A patient's adaptive capacity to cope with the stress of the pathology, diagnosis and prognosis, possible neoadjuvant treatment, surgery, and hospitalization is related to the degree of recovery of physical functioning and, if necessary, the ability to complete subsequent adjuvant treatment following surgery. Before surgery, a patient's adaptive capacity should be determined by performing a physical, nutritional, and cognitive risk assessment to identify a patient's risks for a complicated postoperative course. Preventive strategies (e.g., physical exercise training, nutritional support, psychological support) might be offered to, and executed by the patient, with help of an informal support system. 
Status: state-of-the-art perioperative physical therapy care

A cross-sectional survey was performed in Dutch hospitals to establish routine perioperative physical therapy services for patients undergoing elective abdominal surgery, as well as to analyze the differences between the advised (inter)national versus the reported national 'stateof-the-art' physical therapy (Chapter 2). With a response rate of $79 \%$, hospitals showed a great variability in the implementation and content of preoperative physical therapy practices, while less variability was reported regarding postoperative physical therapy practices. When evaluating the compliance of the self-reported perioperative physical therapy practices with the advised literature at that time (2017), three classes of perioperative physical therapy practices were identified (see Figure 1). Respondents that did not provide preoperative physical therapy were most likely to belong to the conservative class ( $n=30,46 \%)$. The moderately progressive class $(n=19,29 \%)$ and progressive class $(n=16,25 \%)$ both provided preoperative risk assessment, whereas preoperative training was most likely to be offered in the progressive class (Figure 1, column preventive). Only one hospital aligned with more than $75 \%$ of the items for state-of-the-art evidence-based practices as advised in the literature at that time.

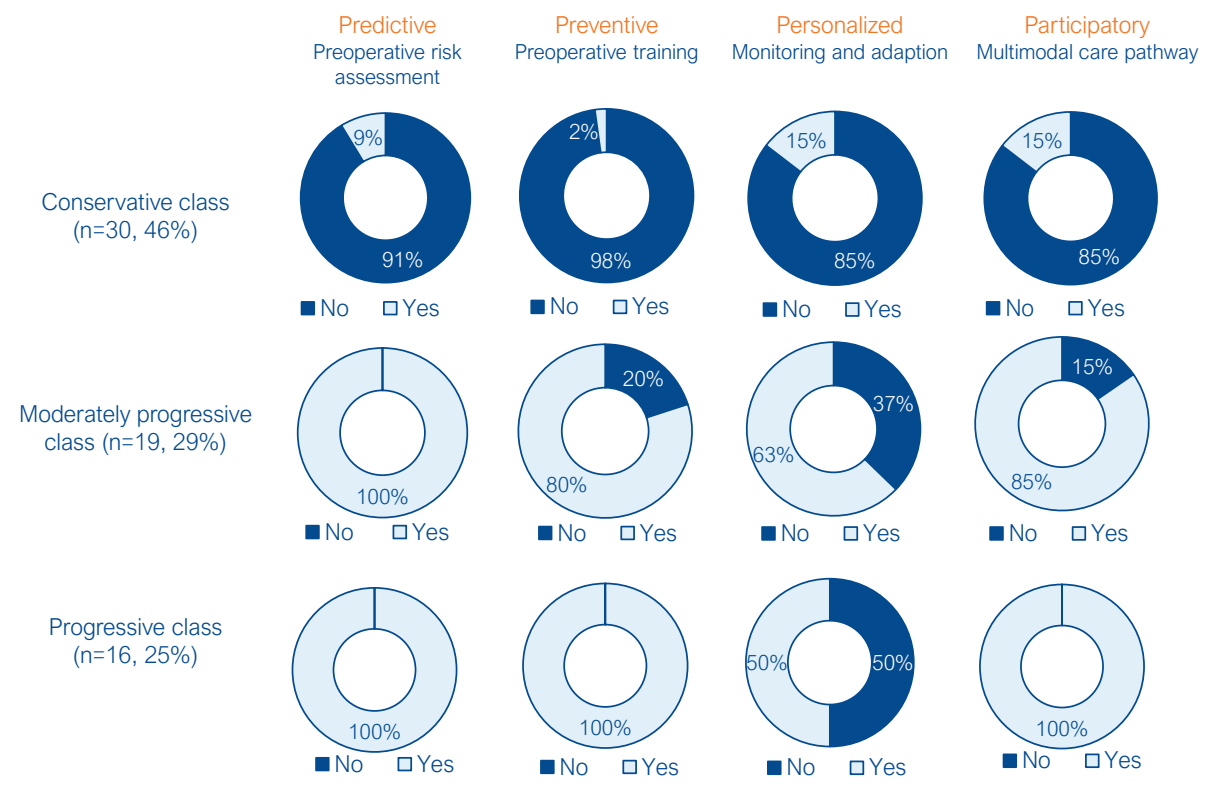

Figure 1. The reported perioperative physical therapy practice divided in three classes and compared on predictive, preventive, personalized, and participatory perioperative physical therapy care. 
Transition towards P4 perioperative physical therapy care

To change perioperative physical therapy practice towards the advised state-of-the-art physical therapy at the local niche level, a transition towards proactive perioperative physical therapy, including components of predictive, preventive, personalized, and participatory care was initiated by an embedded scientist working as a physical therapist (Chapter 3 ). The embedded scientist implemented the state-of-the-art and more recent evidence-based insights in the care pathway of patients with hepatic or pancreatic cancer opting for surgery. At the same time, the embedded scientist observed, monitored, and reflected on the incremental changes in a patient's vital physical functioning in collaboration with patients, health care professionals, and scientists (Figure 2).
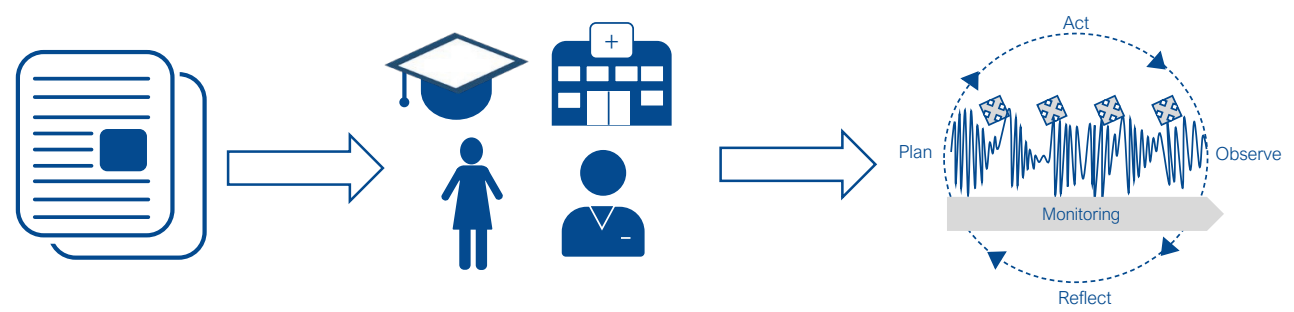

Figure 2. Implementing scientific evidence by building academic partnerships between patients, health care professionals, and scientists, leading to changes in real-life practice with continuous monitoring of a patient's pre- and postoperative physical functioning following a plan-act-observereflect cycle.

In almost four years, the hepato-pancreato-biliary care pathway underwent changes mainly within the context of predictive and preventive care, followed by personalized care. A preoperative assessment unit with risk assessment based on physical functioning and nutritional status was developed, and parameters of preoperative physical functioning related to postoperative outcomes were identified. Furthermore, a trained network with community physical therapists working within the catchment area of the hospital $(n=28)$ was developed to support patients in their own living context to improve their level of physical functioning prior to surgery when necessary. Based on the results of the performance tests that were used to continuously monitor a patient's pre- and postoperative physical functioning, pre- and postoperative physical therapy interventions were adapted in dose, intensity, and type of training. Overall, 174 patients undergoing hepatic or pancreatic resection were pre- and postoperatively monitored. For both types of surgery, median time to recovery of physical functioning decreased ( 1 day in hepatic resection and 2 days in pancreatic resection), and length of hospital stay decreased as well (1 day in hepatic resection and 4 days in pancreatic 
resection). This type of integrating research in daily clinical practice might overcome the gap between the advised (inter)national physical therapy and the reported national 'state-of-the-art' physical therapy.

Monitoring of a patient's perioperative physical functioning

To describe a patient's physical functioning before and after surgery, and to assess which patients might benefit from preoperative preventive strategies, the role of preoperative physical performance-based tests and their association with short-term postoperative outcomes was evaluated (time to recovery of physical functioning, complications, and length of hospital stay) (see Figure 3, Chapter 4 and Chapter 5).

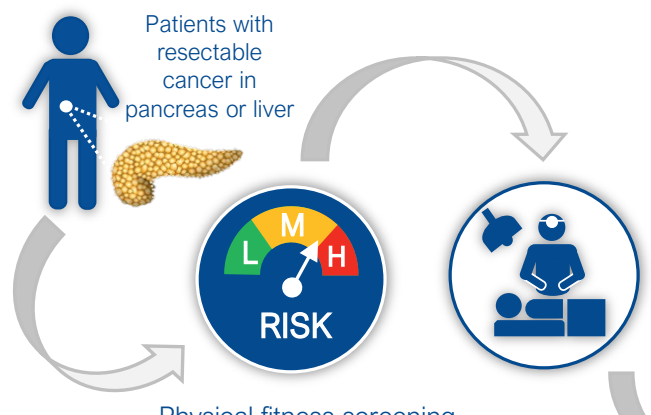

Physical fitness screening

External validation of risk prediction models

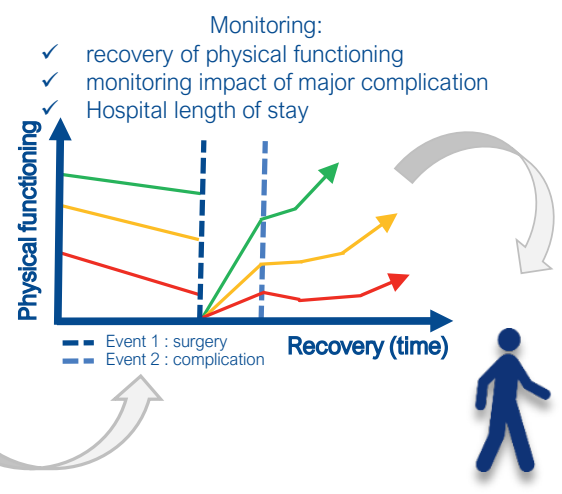

Figure 3. Pre- and postoperative monitoring of physical functioning in patients undergoing elective hepatic or pancreatic resection. Abbreviations: $\mathrm{H}=$ high risk; L=Low risk; $\mathrm{M}=$ =medium risk.

Time to recovery of physical functioning was monitored using the modified lowa level of assistance scale. Overall, 96 patients with hepatic cancer and 63 patients with pancreatic cancer scheduled for resection were pre- and postoperatively monitored. The results showed that a combination of conventional preoperative patient-related characteristics and preoperative performance tests of physical functioning were associated with postoperative outcomes (Table 1) (Chapter 4 and Chapter 5). 
Table 1. Preoperative patient characteristics and performance indicators and their association with postoperative outcomes in patients scheduled for hepatic or pancreatic resection.

\section{Hepatic resection (Chapter 4)}

\begin{tabular}{|c|c|c|}
\hline $\begin{array}{l}\text { Recovery of } \\
\text { physical functioning }\end{array}$ & $\begin{array}{l}\text { Body mass index } \\
\text { American Society of Anesthesiologists score } \\
\text { Aerobic capacity } \\
\text { Perceived level of physical functioning }\end{array}$ & $\begin{array}{l}\text { Steep ramp test } \\
\text { DASI, VSAQ }\end{array}$ \\
\hline $\begin{array}{l}\text { Non-surgical } \\
\text { complications }\end{array}$ & $\begin{array}{l}\text { Body mass index } \\
\text { American Society of Anesthesiologists score } \\
\text { Charlson comorbidity index } \\
\text { Aerobic capacity } \\
\text { Perceived level of physical functioning } \\
\text { Functional mobility }\end{array}$ & $\begin{array}{l}\text { Steep ramp test } \\
\text { DASI, VSAQ } \\
\text { TUG test, FTSTS test, } 2 \mathrm{MWT}\end{array}$ \\
\hline $\begin{array}{l}\text { Length of hospital } \\
\text { stay }\end{array}$ & $\begin{array}{l}\text { Charlson comorbidity index } \\
\text { Perceived level of physical functioning } \\
\text { Functional mobility }\end{array}$ & $\begin{array}{l}\text { DASI, VSAQ } \\
\text { TUG test, FTSTS test, } 2 \mathrm{MWT}\end{array}$ \\
\hline \multicolumn{3}{|c|}{ Pancreatic resection (Chapter 5) } \\
\hline $\begin{array}{l}\text { Recovery of } \\
\text { physical functioning }\end{array}$ & $\begin{array}{l}\text { Age } \geq 70 \text { years } \\
\text { Aerobic capacity } \\
\text { Functional mobility } \\
\text { Perceived level of physical functioning } \\
\text { White blood cell count }\end{array}$ & $\begin{array}{l}\text { Steep ramp test } \\
2 \mathrm{MWT} \\
\text { VSAQ, DASI }\end{array}$ \\
\hline Major complication & $\begin{array}{l}\text { Age } \geq 70 \text { years } \\
\text { American Society of Anesthesiologists score } \\
\text { Platelet count }\end{array}$ & \\
\hline $\begin{array}{l}\text { Impact major } \\
\text { complication on } \\
\text { physical functioning }\end{array}$ & $\begin{array}{l}\text { Age } \geq 70 \text { years } \\
\text { Aerobic capacity } \\
\text { Functional mobility } \\
\text { Perceived level of physical functioning }\end{array}$ & $\begin{array}{l}\text { Steep ramp test } \\
\text { 2MWT } \\
\text { DASI }\end{array}$ \\
\hline
\end{tabular}

Furthermore, the impact of major complications following pancreatic resection was evaluated by exploring the relation with time to recovery of physical functioning in 27 patients experiencing such complications (Chapter 5). Results demonstrated that time to recovery of physical functioning was shorter in younger patients, in those with a higher level of physical functioning (measured at the steep ramp test and two-minute walk test), and in those with a higher perceived level to perform activities of daily life (indicated by the Duke activity status index). Consequently, patients with a higher level of physical functioning might better cope with the consequences of a major complication, as its impact seemed less. Hence, alternative outcome measures should be developed to adequately measure not just the incidence of a complication, but also its impact on a patient's (recovery of) physical functioning to analyze whether these patients benefit from surgery and regain their level of physical functioning after surgery. 


\section{Community prehabilitation with a high-risk patient}

The preoperative period provides a window of opportunity to employ preventive strategies to enhance a patient's level of physical functioning prior to surgery, especially for those patients classified as high-risk based on preoperative risk assessment. A single-subject design was used to explore this option in a high-risk patient prior to pylorus-preserving pancreatoduodenectomy (Chapter 6). Preoperative optimization during a four-week community-based exercise program resulted in an increase in preoperative level of physical functioning. The program was designed with help of the CONTENT-scale to ensure therapeutic validity. The program consisted of aerobic training, resistance training, and functional task training targeted at a moderate exercise intensity twice a week. Titration supported the treatment by monitoring a patient's physical functioning. Shared decision-making between patient and physical therapist was performed regarding dose, intensity, and type of exercises. The results demonstrated a beneficial effect on preoperative level of functional mobility based on several performance tests (see Figure 4).
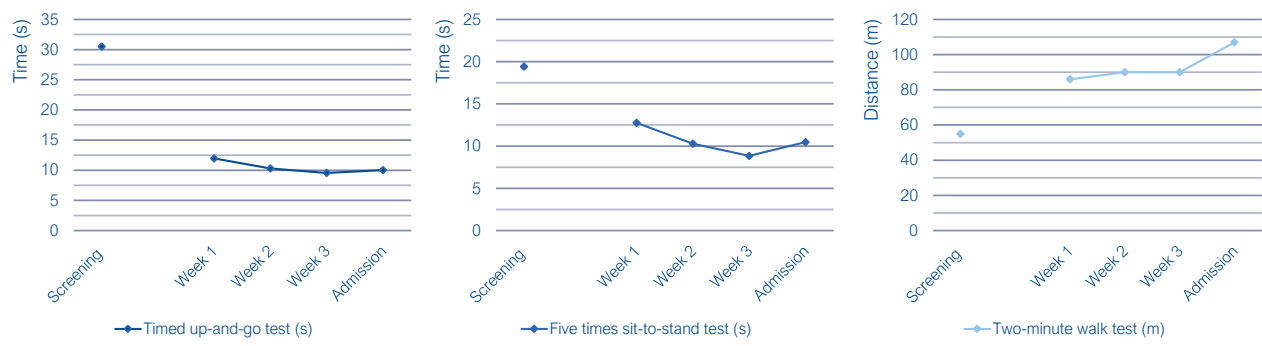

Figure 4. Preoperative performance tests before, during, and after the prehabilitation period in a highrisk patient opting for pylorus-preserving pancreatoduodenectomy.

However, preventive strategies in the context of perioperative care should not only be provided in the preoperative period. To maximally benefit from exercise prehabilitation, it is hypothesized that a physically and mentally activating culture and infrastructure should be provided postoperatively as well. In hospitals, patients are currently mostly "invited" or even - implicitly forced to remain sedentary by the prevailing culture, infrastructure, and professionals, as most care is bed-centered, thereby hampering recovery of physical functioning.

In conclusion, preoperative risk-assessment can support clinical decision-making in a high-risk patient, and the results of the community-based prehabilitation program indicate that in four weeks a remarkable improvement in physical functioning can be made in those patients. 


\section{NEXT - Better Be Prepared}

The pre- and postoperative screening tools, prehabilitation program, and embedded science model developed in perioperative physical therapy care management have been validated in a single university hospital and colleagues in the catchment area. Though considered to be relevant for society, a complete adoption and sustainable implementation of these instruments in daily clinical perioperative physical therapy practice is still lacking on a national level. Nationally, physical therapists should work interdisciplinary and collaborate between sectors to improve the content and context of perioperative physical therapy management, to align on the mission for perioperative care and improve long-term vital functioning of patients undergoing elective abdominal surgery.

\section{BETTER BE PREPARED}

Be inspired by patients, while supporting their adaptation during major life events

Embrace technology and science, and drive change to improve societal impact

Train the right patients, in the right context, at the right time, and with the right support and therapy

Take responsibility to enhance data-management to demonstrate the improvement of patient outcomes

Emerge from an ego-centered system towards an eco-centered system, with full social and professional responsibility

Register the right P4 outcomes, share these, and learn from each other 
"In der Beschränkung zeigt sich erst der Meister".

- J.W. von Goethe's citaat (1802) - 
Samenvatting 



\section{SAMENVATTING}

Tegelijk met grote demografische veranderingen, zorgen technologische en sociaal-culturele innovaties voor veranderingen in de gezondheidszorg. De gezondheidszorg is in transitie, van een min of meer reactieve medische benadering (curatie- en zorggericht) naar een proactieve predictieve, preventieve, persoonlijke en participatieve benadering (P4-health), gericht op het dagelijks functioneren van mensen. Om de transitie te begeleiden is in 2016 een missie-gedreven innovatiebeleid in Nederland gestart. De missie voor de gezondheidszorg zoals geformuleerd in de Kennis en Innovatie Agenda van 2020-2023 is: "in 2040 leven alle Nederlanders tenminste vijf jaar langer in goede gezondheid, en zijn de gezondheidsverschillen tussen de laagste en hoogste sociaal-economische groepen met 30\% afgenomen". Een dergelijke missie vereist veranderingen in de huidige zorgpraktijk en biedt mogelijkheden voor het experimenteren met en het valideren van met elkaar verweven technologische en sociale innovaties met patiënten, hun familieleden, zorgverleners, wetenschappers en ondernemers.

\section{Onze missie}

Het doel van dit missiegedreven proefschrift was om een transitie in de perioperatieve fysiotherapeutische zorg te initiëren van een one-size-fits-all reactieve benadering naar een proactieve P4 perioperatieve fysiotherapeutische benadering voor het behoud van vitaal functioneren voor patiënten die electieve lever- of pancreaschirurgie ondergaan.

\section{Vitaal functioneren voor en na een operatie}

Chirurgie is de primaire curatieve behandeling voor patiënten met kanker in de lever of pancreas. Het is een uitdaging voor patiënten om zelfstandig te blijven functioneren tijdens een 'major life event' zoals een grote operatie en de bijbehorende ziekenhuisopname. Het adaptatievermogen van een patiënt om adequaat om te kunnen gaan met de (psycho)fysiologische stress van de pathologie, de diagnose, de prognose, de mogelijke neoadjuvante behandeling, de chirurgie en de ziekenhuisopname is gerelateerd aan de mate van het herstel van het fysiek functioneren, alsook met het vermogen om de daaropvolgende mogelijke adjuvante behandeling te kunnen voltooien. Het preoperatief in kaart brengen van het fysiek functioneren, de voedingsstatus en de cognitieve status van een patiënt geeft een indruk van het vermogen van een patiënt om adequaat te reageren op deze stressoren. Preoperatieve preventieve interventies, zoals fysieke training, voedingsondersteuning en/of psychologische ondersteuning in de leefomgeving van de patiënt beogen de (psycho)fysiologische reservecapaciteit van een patiënt te verbeteren indien deze onvoldoende lijkt. 
Variatie in de huidige perioperatieve fysiotherapeutische zorg

Om na te gaan hoe de perioperatieve fysiotherapie in 2017 in Nederland was ingericht voor patiënten die electieve abdominale chirurgie ondergaan, is er een vragenlijststudie onder 82 Nederlandse ziekenhuizen uitgevoerd (Hoofdstuk 2). Tegelijkertijd kon zo geanalyseerd worden wat de verschillen waren tussen de geadviseerde (inter)nationale state-of-the-art fysiotherapie en de gerapporteerde huidige fysiotherapie. Met een responspercentage van $79 \%$ vertoonden Nederlandse ziekenhuizen een grote variabiliteit in de implementatiestatus en inhoud van de preoperatieve fysiotherapie. Bij het evalueren van de conformiteit van de huidige praktijk met de geadviseerde (inter)nationale fysiotherapie werden er drie klassen in de huidige fysiotherapeutische zorg geïdentificeerd (zie Figuur 1, conservatief, matig progressief en progressief). Bevraagde ziekenhuizen die geen preoperatieve fysiotherapie aanboden aan patiënten in hun dagelijkse praktijk, hadden de grootste kans om tot de conservatieve klasse te behoren ( $n=30,46 \%)$. Ziekenhuizen behorende tot de matig-progressieve klasse ( $n=19,29 \%)$ en progressieve klasse $(n=16,25 \%)$ hadden een preoperatieve screening opgenomen in hun zorgpad waarvan ziekenhuizen die prevalidatie aanboden de grootste kans hadden om tot de progressieve klasse te behoren. Slechts in één ziekenhuis kwam de dagelijkse praktijk overeen met $>75 \%$ van de geadviseerde state-of-the art literatuur.

Conservatief $(n=30,46 \%)$

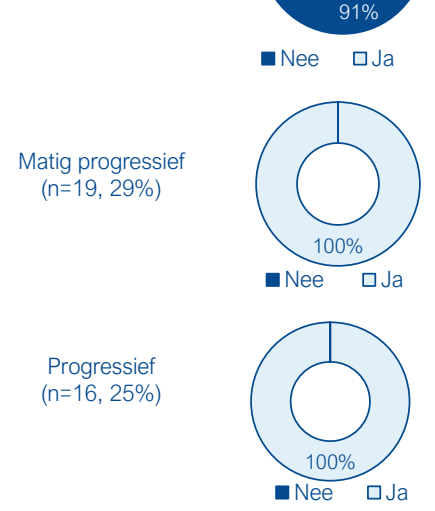

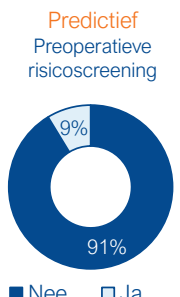
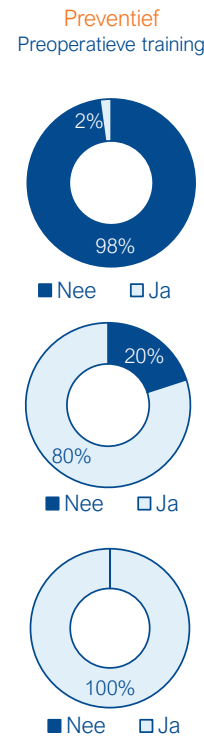
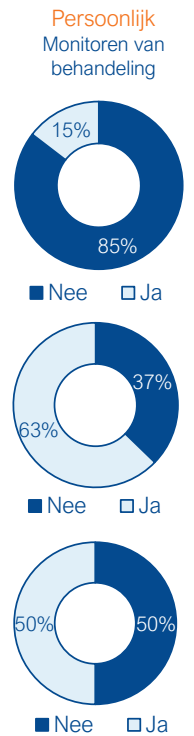

Participatief

Multidisciplinaire en

multimodale behandeling

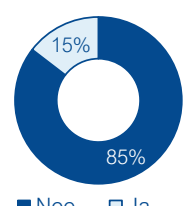

- Nee $\square \mathrm{Ja}$
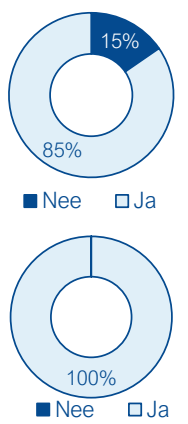

Figuur 1. De gerapporteerde pre- en postoperatieve fysiotherapie onderverdeeld in drie klassen en vergeleken op de items over predictie, preventie, persoonlijk en participatie binnen de perioperatieve fysiotherapeutische zorg. 
Om de huidige perioperatieve fysiotherapie in de richting van de geadviseerde state-of-the-art fysiotherapie op het lokale niche-niveau te veranderen, werd een transitie naar proactieve perioperatieve fysiotherapie geïnitieerd. Tijdens deze transitie werd er bijzonder aandacht besteed aan predictie, preventie, persoonlijk en participatie (Hoofdstuk 3). Een 'embedded scientist' implementeerde de state-of-the-art en recentere evidence-based inzichten in het zorgtraject van patiënten met lever- of pancreaskanker welke besloten hadden om een (electieve) operatie te ondergaan. Tegelijkertijd observeerde, monitorde en reflecteerde de 'embedded scientist' de veranderingen in het fysiek functioneren van patiënten samen met de patiënt, familie, gezondheidsprofessionals en wetenschappers (zie Figuur 2).
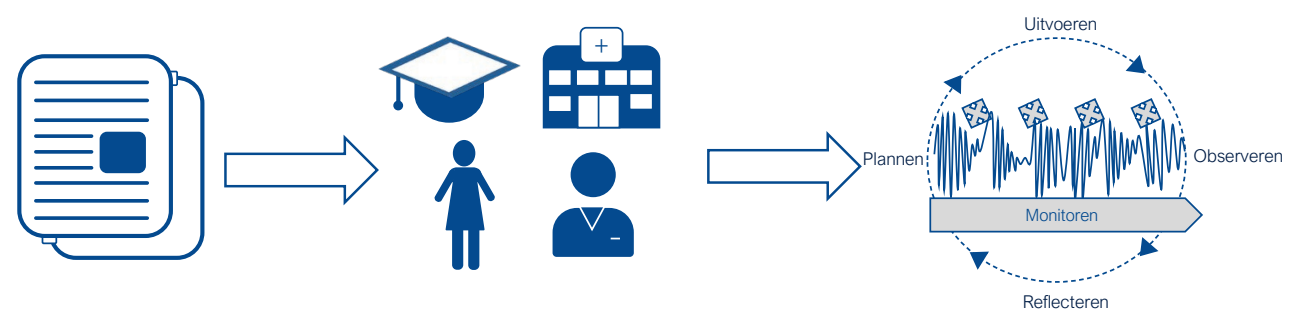

Figuur 2. Het implementeren van wetenschappelijk bewijs in de zorg door het opzetten van academische partnerships met patiënten, zorgprofessionals en wetenschappers. Door het volgen van een continue cyclus van plannen, uitvoeren, observeren en reflecteren en het continue monitoren van het fysiek functioneren kon een verandering worden waargenomen in de real-life klinische zorgcontext.

In bijna vier jaar tijd onderging de fysiotherapeutische perioperatieve hepato-pancreato-biliaire zorg voornamelijk veranderingen in de context van predictieve en preventieve zorg, gevolgd door gepersonaliseerde zorg. Een preoperatieve screening van het fysiek functioneren en voedingsstatus werden ontwikkeld en parameters van het preoperatief fysiek functioneren geassocieerd aan postoperatieve uitkomsten werden geïdentificeerd. Een eerstelijns prevalidatienetwerk van fysiotherapeuten die werkzaam waren in de omgeving van het ziekenhuis $(n=28)$ werd opgezet om patiënten in hun eigen leefomgeving te ondersteunen om - indien geïndiceerd - voorafgaand aan de operatie hun fysiek functioneren te optimaliseren door gesuperviseerde fysieke training in de thuiscontext. Het pre- en postoperatief monitoren van het fysiek functioneren van een patiënt resulteerde in gepersonaliseerde pre- en postoperatieve fysiotherapie. 
In totaal werden 174 patiënten die een resectie van de lever of pancreas ondergingen pre- en postoperatief gevolgd. Voor beide patiëntpopulaties reduceerde de mediane tijd tot het postoperatief herstel van het fysiek functioneren (-1 dag na leverresectie en -2 dagen na pancreasresectie) en de mediane ziekenhuisopnameduur (-1 dag na leverresectie en -4 dagen na pancreasresectie). Dit type onderzoek, dat wetenschap integreert met de dagelijkse klinische praktijk, is een manier om de kloof te kunnen overbruggen tussen de geadviseerde (inter)nationale state-of-the-art fysiotherapie en de gerapporteerde huidige nationale fysiotherapie.

\section{Monitoren van het pre- en postoperatief fysiek functioneren}

Om het fysiek functioneren van een patiënt voor en na de operatie te beschrijven, en om te beoordelen welke patiënten baat kunnen hebben bij preoperatieve preventieve interventies, werd gekeken welke preoperatieve parameters van het fysiek functioneren gemeten met prestatiegebaseerde tests geassocieerd waren met korte termijn postoperatieve uitkomsten (tijd tot herstel van het fysiek functioneren, complicaties, en duur van ziekenhuisopname) (Hoofdstuk 4 en Hoofdstuk 5).

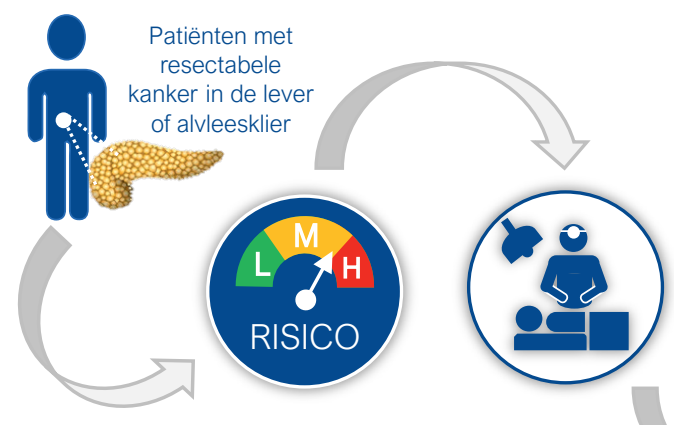

Monitoren:

$\checkmark$ Herstel van het fysiek functioneren

$\checkmark$ De impact van een ernstige complicatie

$\checkmark$ Duur van ziekenhuisopname

Screenen van het fysiek functioneren

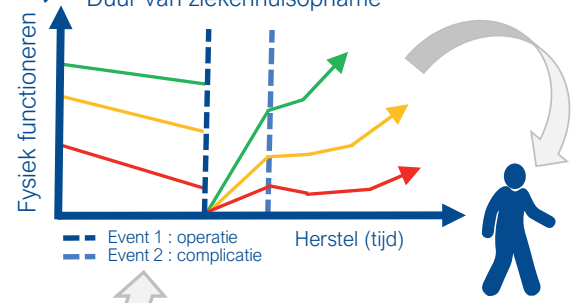

Figuur 3. Pre- en postoperatieve monitoring van het fysiek functioneren van patiënten die electieve lever- of pancreasresectie ondergaan.

Afkortingen: $\mathrm{H}=$ hoog-risico; L=laag-risico; $\mathrm{M}=$ gemiddeld risico.

Het postoperatief herstel van het fysiek functioneren werd gemonitord met behulp van de 'modified lowa level of assistance scale'. Bij 96 patiënten met kanker in de lever en 63 patiënten met kanker in de pancreas waren naast de klassieke risicofactoren ook parameters van het fysiek functioneren geassocieerd met postoperatieve uitkomsten (zie Tabel 1). 
Tabel 1. Preoperatieve patiëntkarakteristieken en parameters van het fysiek functioneren en de associatie hiervan met postoperatieve uitkomsten in patiënten die chirurgie ondergingen voor kanker in de lever of pancreas.

\section{Leverresectie (Hoofdstuk 4)}

Herstel van fysiek

functioneren

Niet-chirurgische
complicaties

Opnameduur
Body mass index

American Society of Anesthesiologists score Inspanningscapaciteit

Ervaren level van dagelijks fysiek functioneren

Body mass index

American Society of Anesthesiologists score

Charlson comorbidity index

Inspanningscapaciteit

Ervaren level van dagelijks fysiek functioneren

Functionele mobiliteit

Charlson comorbidity index

Ervaren level van dagelijks fysiek functioneren

Functionele mobiliteit
Steep ramp test

DASI, VSAQ

Steep ramp test

DASI, VSAQ

TUG test, FTSTS

test, 2MWT

DASI, VSAQ

TUG test, FTSTS

test, 2MWT

\section{Pancreasresectie (Hoofdstuk 5)}

Herstel van fysiek functioneren

Ernstige complicatie $(C D C \geq 3)$

Impact van ernstige complicatie op postoperatief herstel van het fysiek functioneren
Leeftijd $\geq 70$ jaar

Aerobic capacity

Ervaren level van dagelijks fysiek functioneren

Functionele mobiliteit

Aantal witte bloedcellen

Steep ramp test

VSAQ, DASI

2MWT

Afkortingen: CDC=Clavien-Dindo classificatie; DASI=Duke activity status index; FTSTS=five times sit-to-stand; TUG=timed up-and-go; VSAQ=veterans-specific activity questionnaire; 2MWT=twee-minuten wandeltest.

Verder werd de impact van een ernstige complicatie geëvalueerd door de invloed hiervan op de tijd tot herstel van het fysiek functioneren te onderzoeken bij de 27 patiënten die een ernstige complicatie hadden ervaren na een pancreasresectie (Hoofdstuk 5). Resultaten toonden aan dat de tijd tot het herstel van het fysiek functioneren korter was bij jongere patiënten, bij patiënten met een hoger niveau van fysiek functioneren (betere prestatie op de steep ramp test en wandeltest) en bij patiënten met een hoger ervaren level van het dagelijks fysiek functioneren (Duke activity status index). Patiënten met een beter fysiek functioneren lijken beter om te kunnen 
gaan met de gevolgen van een ernstige complicatie, omdat de impact op het fysiek functioneren minder lijkt. Met deze bevindingen zal in toekomstig onderzoek verder gekeken moeten worden naar alternatieve uitkomstmaten om niet alleen de incidentie van een complicatie te registreren, maar ook de impact hiervan op het (herstel van het) fysiek functioneren van een patiënt te evalueren.

\section{Prevalidatie van een patiënt met een hoog-risico profiel}

De preoperatieve periode bij electieve chirurgie biedt een mogelijkheid om preventieve interventies in te zetten om het niveau van het fysiek functioneren van een patiënt voorafgaand aan de operatie te verbeteren. Met name patiënten met een hoog-risico profiel op basis van de preoperatieve screening lijken hier baat bij te hebben. Om de effecten van preoperatieve training in een eerste-lijn fysiotherapiepraktijk bij een patiënt die een pylorus-preservingpancreaticoduodenectomy opteerde met een hoog-risico profiel te evalueren (Hoofdstuk 6) werd een single-subject design gebruikt. Een gesuperviseerd (twee keer per week) preoperatief trainingsprogramma in de woonomgeving van de patiënt resulteerde in een verbetering van het preoperatief fysiek functioneren gemeten met diverse prestatie-gebaseerde tests (Figuur 4). Het programma was ontworpen met behulp van de CONTENT-schaal om de therapeutische validiteit te garanderen en bestond uit aërobe training, krachttraining en het trainen van functionele taken. Titratie ondersteunde de behandeling door het monitoren van het fysiek functioneren van de patiënt en gedeelde besluitvorming tussen patiënt en therapeut over de dosis, de intensiteit en de type oefeningen van de therapie.
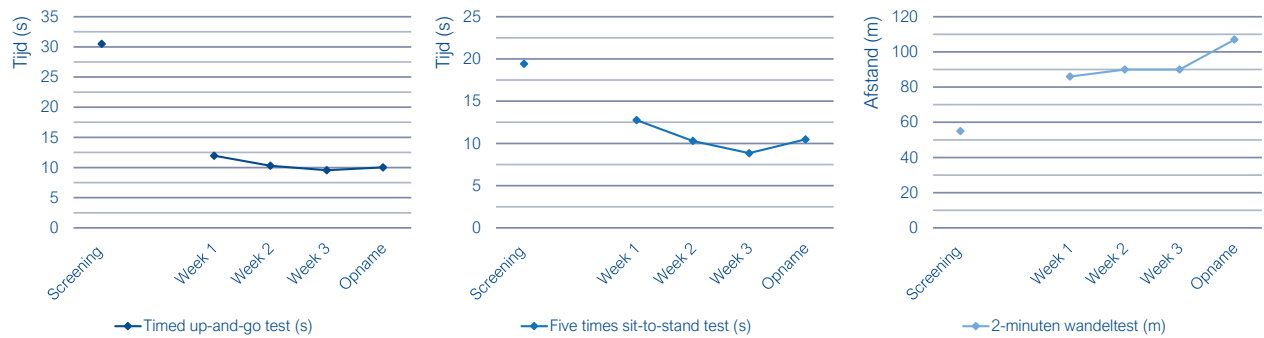

Figuur 4. Preoperatieve prestatie-gebaseerde tests afgenomen op de preoperatieve screening, aan het begin van elke prevalidatie week en bij opname in het ziekenhuis van een hoog-risico patiënt om de effecten van prevalidatie te evalueren. 
Preventieve interventies voor het verbeteren van de perioperatieve zorg dienen niet alleen in de preoperatieve periode te worden aangeboden. Om maximaal profijt te hebben van prevalidatie is een postoperatieve fysiek en mentaal activerende cultuur en infrastructuur ook van belang. In ziekenhuizen worden patiënten 'uitgenodigd' om inactief te blijven, omdat de ziekenhuiscontext (de heersende cultuur en infrastructuur) ervoor zorgt dat patiënten niet uitgedaagd worden om 'actief' te werken aan hun eigen herstel van het fysiek functioneren.

Concluderend kan gesteld worden dat een preoperatieve screening zowel de patiënt als de zorgprofessional kan ondersteunen in de klinische besluitvorming voor het ondergaan van de operatie en daaraan voorafgaand het volgen van prevalidatie. Een gesuperviseerd prevalidatie programma van een hoog-risico patiënt in de eigen woonomgeving laat zien dat verbetering van het fysiek functioneren in een korte tijdsperiode mogelijk is.

\section{Better Be Prepared}

De instrumenten (de pre- en postoperatieve screeningsinstrumenten, het prevalidatieprogramma en het 'embedded science' model) ontwikkeld in de klinische fysiotherapeutische zorgcontext zijn gevalideerd in één ziekenhuis en zijn omgeving. Hoewel deze relevant geacht worden voor de samenleving, ontbreekt een volledige acceptatie en duurzame implementatie van deze instrumenten in de dagelijkse klinische perioperatieve fysiotherapie op nationaal niveau. Fysiotherapeuten dienen op nationaal niveau interdisciplinair en sectoroverstijgend te werk te gaan om de inhoud en de context van pre- en postoperatieve fysiotherapie te optimaliseren, en om zich aan te passen aan de missie voor de perioperatieve zorg om het vitaal functioneren op de korte en lange termijn van patiënten die electieve chirurgie ondergaan te verbeteren.

\section{BETTER BE PREPARED}

Be inspired by patients, while supporting their adaptation during major life events

Embrace technology and science, and drive change to improve societal impact

Train the right patients, in the right context, at the right time, and with the right support and therapy

Take responsibility to enhance data-management to demonstrate the improvement of patient outcomes

Emerge from an ego-centered system towards an eco-centered system, with full social and professional responsibility

Register the right P4 outcomes, share these, and learn from each other 
Coming together is a beginning, staying together is progress, and working together is success.

- Henry Ford (1863-1947) - 
Dankwoord 



\section{Chaos bedankt!}

Chaos is iets wat we in het dagelijks leven zoveel mogelijk proberen te vermijden. We streven immers altijd naar orde en gaan chaos uit de weg, omdat het wordt geassocieerd met turbulentie, onvoorspelbaarheid en instabiliteit. Echter is het maar de vraag of dat laatste wel waar is. In de wiskunde wordt chaos namelijk gezien als schijnbare wanorde: de dynamiek, ofwel het gedrag, is onvoorspelbaar, maar deze komt tot stand door een bepaald algoritme (wetmatigheden). Kortom, het gedrag van een chaotisch proces is onvoorspelbaar, maar de interacties tussen de verschillende elementen - de diverse personen in het systeem - bepalen de uitslag van die dynamiek. Tijdens een promotietraject maak je de dynamiek van een chaotisch proces mee. Je verandert meermaals van koers, krijgt gaandeweg nieuwe inzichten en de omgeving verandert continu. Door de interactie met patiënten, zorgprofessionals, collega's, promotoren, co-promotor en mijn naasten lukte het me om me telkens aan te passen aan de chaotische omstandigheden binnen dit promotietraject. Ondanks dat eenieders adaptief vermogen flink op de proef is gesteld tijdens dit traject, mag ik bij het schrijven van dit dankwoord wel stellen dat we chaos mogen omarmen. Chaos heeft ons namelijk gezamenlijk in staat gesteld om zorg en onderzoek te combineren om zo de fysiotherapeutische zorg voor patiënten die een grote buikoperatie ondergaan voor kanker in de lever of alvleesklier te veranderen, zoals beschreven in dit proefschrift.

Ik wil graag iedereen bedanken die op zijn of haar eigen wijze heeft bijgedragen aan deze chaos en de totstandkoming van dit proefschrift: bedankt! Een aantal personen wil ik graag in het bijzonder op een 'chaotische' manier bedanken

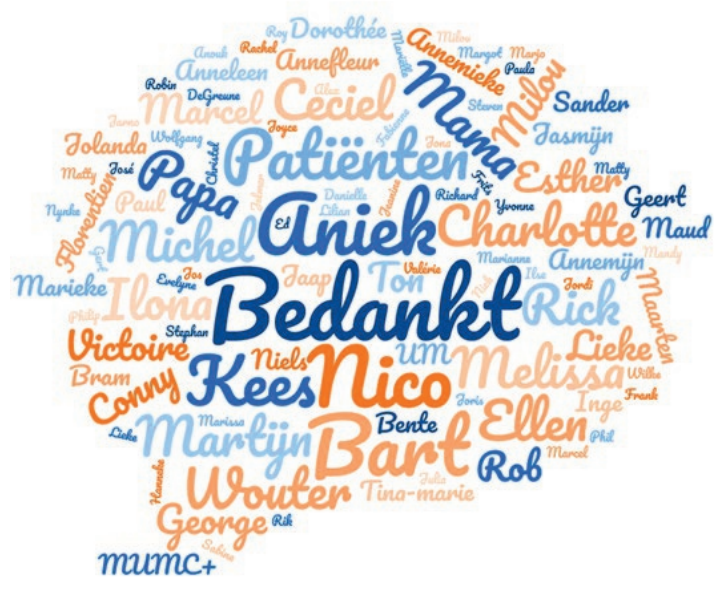


Allereerst wil ik alle patiënten bedanken. Als beginnende zorgprofessional heb ik veel van jullie mogen leren en vond ik het een voorrecht dat jullie ervaringen met mij wilden delen om het proces voor en na de operatie vervolgens samen met jullie te optimaliseren. Dit was een extra stimulans om elke dag mijn witte pak aan te doen en dit onderzoek uit te voeren.

Prof.dr. Nico van Meeteren, prof.dr. Kees Dejong en dr. Bart Bongers, bedankt voor jullie begeleiding tijdens dit leerzame promotietraject. Jullie waren een bron van inspiratie, creëerden mogelijkheden en ik heb ontzettend veel van jullie mogen leren.

\section{Prof.dr. Nico van Meeteren, beste Nico,}

Uw bevlogenheid en passie voor de fysiotherapiewetenschap, en hoe $u$ deze inzet voor maatschappelijke uitdagingen, is bewonderingswaardig. Uw scherpe focus, visie en creatieve kijk op zaken werkte aanstekelijk en motiverend. Na elk overleg keerde ik vaak terug met chaotische schetsen voor een figuur, diverse theorieën waarvan ik de samenhang op dat moment vaak nog niet zag, nieuwe woordspelingen en vocabulaire en een dosis energie om weer aan de slag te gaan. $U$ was altijd bereid om te bellen, keek altijd zeer kritisch naar mijn stukken en zorgde ervoor dat we op koers bleven. Verder steunde $u$ me in mijn plannen en ideeën en toonde $u$ altijd persoonlijke betrokkenheid, niet alleen voor mijn ontwikkeling als professional, maar ook in mij als persoon. Hartelijk dank dat ik zoveel van u heb mogen leren en uw Pipi Langkous filosofie zal me altijd bijblijven.

\section{Prof.dr. Kees Dejong, beste Kees,}

Uw pragmatisme en doelgerichtheid hielpen mij in het ziekenhuis mijn weg te vinden binnen de HPB-chirurgie. $U$ was altijd genegen mij te helpen, kritische feedback te geven op mijn artikelen en mij van nuttige adviezen te voorzien. Zo bedacht u het stoplichten schema om inzicht te krijgen in de voortgang van de diverse hoofdstukken van mijn proefschrift en deelde u met regelmaat uw ervaringen uit uw eigen promotietijd. Verder kreeg ik door $u$ de kans om mijn onderzoek ook meermaals internationaal te presenteren op het E-HPBA congres, hetgeen mij hielp om kritische vragen over mijn onderzoek te beantwoorden.

\section{Dr. Bart Bongers, beste Bart,}

Meermaals schreef of zei je 'In der Beschränkung zeigt sich erst der Meister' als ik weer extreem lange zinnen had opgenomen in mijn manuscript of een naar mijn mening interessant topic in een hele alinea had uitgelicht. In vele gevallen heb ik me daaraan proberen te houden maar in dit 
hoofdstuk werd het toch een stukje lastiger, zeker toen ik bij jouw paragraaf belandde. Ik wil je graag bedanken voor je dagelijkse begeleiding, het delen van je kennis en kunde, je humor en jouw extreem goed oog voor detail. Je positieve instelling in combinatie met de nodige structuur en praktische tips hielpen me altijd weer op weg als ik weer vast zat. Om het in de prachtige wielertaal te zeggen - een sport waartoe jij me gezet hebt - je zorgde ervoor dat ik nooit een 'hongerklop' kreeg of 'geparkeerd' stond en zo in de juiste condities de top van de 'col' bereikte en niet eindigde in een 'chasse patate'.

\section{Dr. Aniek Heldens, beste Aniek,}

Aniek, 4 jaar geleden begonnen we samen aan dit avontuur. Jij was al wat meer ervaren als 'embedded scientist' in het Maastricht UMC+. Je nam me mee op pad en introduceerde me binnen de diverse afdelingen. Het was ontzettend leuk en fijn om met jou samen te mogen werken. Je praktische en georganiseerde aanpak en je nuchtere doe-maar-gewoon-dan-doe-je-al-gek genoeg mentaliteit heb ik altijd in je bewonderd. Verder wist je als kamergenootje altijd goed om te gaan met mijn soms ietwat chaotische aanpak of vond je het niet erg als ik de muren volplakte met A3 schema's, post-its en artikelen. Jij bent een kei in het hanteren van het principe van zorg op de juiste plek, op het juiste moment en bij de juiste persoon, zeker op het gebied van het zorgen voor eten. In het rode ladekastje had je altijd voldoende proviand opgeslagen (waarvan $80 \%$ chocolade) om mij te voorzien van diverse lekkernijen. Buiten het werk om heb ik ook erg genoten van de vele etentjes, borrels, en thee-drink momentjes.

\section{Dr. Marcel den Dulk en Lieke Corpelijn, beste Marcel en Lieke,}

Jullie zorgden er al gauw voor dat ik me thuis voelde binnen de HPB chirurgie. Vanaf het begin waren jullie geïnteresseerd en hebben jullie meegedacht in diverse mogelijkheden voor het optimaliseren van het perioperatieve zorgpad. Marcel, officieel was je geen co-promotor maar ik mocht jou altijd consulteren voor klinische vraagstukken, het interpreteren van de onderzoeksresultaten en het vertalen van de data naar de dagelijkse praktijk. Ik wil je hartelijk danken voor je klinische input en kritische blik op mijn artikelen, en ik vond het heel fijn om met jou samen te werken. Lieke, jij was de 'coördinator van de chaos'. Je was gelijk enthousiast en bereid om mee te denken aan het opzetten van een preoperatieve screeningspoli voor fysieke fitheid, voeding en kwetsbaarheid (de POS-poli). Dit ondanks de drukke poli, de vele brieven en mails die je altijd had liggen. We startten klein, met alleen een fysieke fitheidscreening, maar breidden langzaam uit naar een vaste poli op de donderdagmiddag voor een screening voor kwetsbaarheid, voeding en fysieke fitheid. Jullie hebben beide een onmisbare rol gespeeld in de 
opzet en uitvoering van de POS-poli en ik ben blij dat we dit ook na mijn promotieonderzoek mogen continueren.

Dhr. George Roox, beste George,

Graag wil ik u bedanken voor uw interesse, vertrouwen en aanmoediging in de periode van mijn promotie. $U$ heeft zich altijd opgeworpen als sparring-partner en meegekeken en meegedacht over hoe we op een praktische manier onderzoek konden inbedden in de dagelijkse praktijk. Ik heb veel van uw mogen leren over het vak, de afdeling en de organisatie in de eerste twee jaar van mijn loopbaan. Ondanks uw pensioen was ik gelukkig mijn sparring-maatje niet kwijt, want we hebben er regelmatig nog eentje gedronken in Maastricht en ik hoop dat er nog velen mogen komen.

Graag wil ik de leden van de leescommissie prof.dr. W.F.F.A. Buhre (voorzitter), prof.dr. S.W.M. Olde Damink, prof.dr. L.P.S. Stassen, prof.dr. P.J. van der Wees en prof.dr. R. van Hillegersberg bedanken voor het lezen en beoordelen van dit proefschrift.

\section{De afdeling fysiotherapie van het Maastricht UMC+}

De collega's van de afdeling fysiotherapie van het Maastricht UMC+ wil ik bedanken voor de interesse, aanmoediging en ondersteuning die ik de afgelopen 4 jaar heb mogen ontvangen. Meermaals creëerde ik op de afdeling wat chaos ten aanzien van nieuwe klinimetrie, leanprojecten of stond ik verkeersleider te spelen op de gang tijdens de wandeltests. Allen hartelijk dank voor jullie steun! In het bijzonder wil ik prof.dr. Ton Lenssen en Rob Roomans bedanken voor de ruimte en het vertrouwen dat ik heb mogen krijgen om onderzoek een plaats te geven binnen de afdeling fysiotherapie. Verder heb ik geleerd dat een goed lopend secretariaat essentieel is voor het goed laten lopen van je onderzoek. Victoire Loffeld, Ilse Luijten en Wilke Ellenbroek, ik wil jullie hartelijk danken voor al jullie inspanningen om patiënten te woord te staan en achter de schermen veel te regelen voor mijn onderzoek (scholingen, contracten, onderzoekstagiaires, et cetera). Onderzoek doen met slecht materiaal is niet mogelijk. Margot Jansen en Frank Ramakers, ik wil jullie dan ook hartelijk danken voor het verzorgen van mijn onderzoeksuitrusting (de fiets, stopwatch, saturatiemeters, klappers, folders, et cetera). Verder wil ik ook in het bijzonder Maud Ploumen, Niels Groen, Roy Ridder, Yvonne Feijts, en Marissa Gerards bedanken voor jullie enthousiasme en hulp bij het opzetten van de prevalidatie en de preoperatieve screeningen. 
Verder heb ik veel steun en hulp mogen ontvangen van veel collega's van diverse afdelingen binnen en buiten het ziekenhuis. Collega's van de verpleegafdeling A4 en B4, diëtetiek, heelkunde, geriatrie, anesthesiologie, oncologie, poli-medewerkers, afdelingsmanagers, fysiotherapeuten uit de eerste lijn en anderen die betrokken waren bij het optimaliseren van de perioperatieve zorg voor patiënten die grote abdominale chirurgie ondergaan, ik wil jullie hartelijk danken voor jullie hulp, interesse en bijdrages in de diverse projectgroepen.

Dr. Ilona Punt, Esther Janssen, Conny de Zwart, prof.dr. Rob de Bie, beste Ilona, Esther, Conny en Rob,

Op de achtergrond waren jullie alle vier op jullie eigen manier betrokken bij mijn promotietraject vanuit de universiteit. Ilona, bij jou kon ik met regelmaat binnenlopen met een vraag, advies of gewoonweg om even gezellig te kletsen onder het genot van een kopje thee. Achter de schermen verzette je bergen werk om ervoor te zorgen dat er een prevalidatie-netwerk kwam, scholingen werden opgezet en een beslisondersteuning werd geschreven. Je precisie, bescheidenheid en je harde arbeidsethos siert je en je was een voorbeeld voor mij als jonge onderzoeker. Esther, bedankt voor jouw nuchtere kijk op onderzoek en voor de gezellige lunches, koffiemomentjes en treinritjes naar Utrecht. Conny, zonder jou waren we toch verloren op de UNS40 en later op DEB1. Hartelijk dank voor de gezellige praatjes, treinkaarten, brieven en alle andere praktische tips die ik van je heb mogen ontvangen tijdens mijn promotietraject. Rob, u was altijd in voor een praatje en een grapje. Hartelijk dank voor uw interesse in mijn promotietraject. Verder wil ik graag de andere collega's van de vakgroep Epidemiologie bedanken voor de interesse en support.

\section{Dr. Sander van Kuijk, beste Sander,}

Meermaals ben ik bij je langs mogen komen voor vragen over de methodologie en de statistiek. We namen vaak mijn 'plakboek' met de resultaten van de betreffende studie door, en op een begrijpelijke manier voorzag je me van tips en adviezen op het gebied van statistiek. Ik heb veel van je mogen leren en wil je hartelijk bedanken voor je hulp.

Verder wil ik de leden van de Better in, Better out Community-of-Practice bedanken voor de interessante en gezellige avonden in Utrecht. Ik heb bijzonder genoten van het voorrecht om voorzitter te mogen zijn en jullie bij tijd en wijle tot halt te mogen roepen bij enthousiaste discussies die onze drukke agenda niet altijd kon verdragen. 
Tenslotte wil ik graag mijn vrienden en familie bedanken. Op de juiste momenten stonden jullie voor me klaar voor een advies, ontspanning, plezier en ook relativering. Een aantal wil ik in het bijzonder noemen. Milou, bedankt voor alle leuke momenten, gezelligheid en aanmoediging. Door jouw vriendschap begon ik me 'thuis' te voelen in Maastricht. Dorothée, Anneleen, Inge, Bram en Maarten, ondanks dat ik weer terugverhuisde naar Holland hebben we altijd goed contact onderhouden. Ik wil jullie bedanken voor de gezellige en leuke appjes, telefoongesprekken, stedentripjes, dinertjes en ook voor de interesse en discussies die we hebben mogen voeren aangaande mijn doctoraat. Marieke, jij verbouwde eigenhandig een heel huis samen met Paul gedurende mijn promotietraject, maar maakte altijd tijd voor een telefoontje of een bezoek aan Maastricht. Je enthousiasme en energie is aanstekelijk en ik ben blij en dankbaar voor jouw vriendschap. Annemieke, Jasmijn, en Tina-Marie, in februari begonnen we gezamenlijk aan onze master Bedrijfskunde aan de Universiteit van Amsterdam en jullie verklaarde me vanaf dag één gelijk voor gek. De programmamanager had toch gezegd de komende twee jaar beter niet te verhuizen, trouwen, van baan switchen... Hoorde promoveren niet ook in dat rijtje thuis? Ik heb veel support van jullie mogen ontvangen en kijk ik altijd weer uit naar de vrijdagen met leuke lessen en gezellige borrels.

Papa, mama, (schoon)broers en (schoon)zussen, ieder van jullie heeft op zijn of haar manier bijgedragen aan dit proefschrift, en zonder jullie steun en belangstelling was het nooit gelukt om dit proefschrift succesvol af te ronden. Papa en Mama, bedankt voor jullie liefde, goede raad, en vertrouwen om nooit op te geven en me te ondersteunen en te stimuleren in mijn keuzes voor mijn studie en loopbaan. Verder wil ik jullie bedanken voor het fijne thuis in Dongen. Altijd een gezellige chaos met humor, heerlijke diners met een glaasje, leuke conversaties en belangstelling voor eenieders studie, loopbaan en gezin. Michel, Ellen, Martijn, Wouter en Charlotte, dichtbij of heel ver weg, altijd hebben we veel contact en tonen jullie interesse in mij, mijn studie en mijn werk. Het proces van een proefschrift maken was voor ons allen wat onbekend, en dat leverde soms leuke opmerkingen en gesprekken op. Bijvoorbeeld: 'Je bent al zo lang bezig aan dat artikel, het kan toch niet zó moeilijk zijn om een artikel te schrijven?' Helaas soms wel, maar door jullie hulp bij diverse projecten en de zorg voor tijdige afleiding heb ik er de vaart in kunnen houden. Ik ben blij met jullie als broers en zussen en dankbaar voor alles wat ik al heb mogen ondernemen en leren met jullie. De afgelopen jaren is de familie flink uitgebreid en wil ik ook mijn schoonzussen, -broer, en nichtjes bedanken voor jullie gezelligheid en interesse gedurende mijn promotietraject.

Chaos daar was het allemaal mee begonnen. ledereen bedankt voor het creëren van de chaos die nodig was om mezelf te ontwikkelen en te komen tot dit proefschrift. 


\section{Bedankt!}

Mahalo

Arigatô

Takk

Kop khun

Dzięki
Tack

Grazie
Grazzi

Obrigado

Gracias
Diolch 
A smile puts you on the right track [.....] when you lose your smile, you lose your way in the chaos of life.

- Robert T. Bennett (1939-2014) - 
About the author

Christel van Beijsterveld 


\section{LIST OF PUBLICATIONS}

Internationally peer-reviewed scientific publications

Van Beijsterveld CA, Heldens AF, Bongers BC, Van Meeteren NL. Variation in preoperative and postoperative physical therapist management of patients opting for elective abdominal surgery. Phys Ther. 2019;99:1291-303.

Van Beijsterveld CA, Bongers BC, Den Dulk M, Van Kuijk SM, Dejong CH, Van Meeteren NL. The association between preoperative physical functioning and short-term postoperative outcomes: a cohort study of patients undergoing elective hepatic resection. HPB (Oxford). 2019;21:1362-70.

Van Beijsterveld CA, Bongers BC, Den Dulk M, Van Kuijk SM, Dejong CH, Van Meeteren NL. Exploring the relation between preoperative physical functioning and the impact of major complications in patients following pancreatic resection. HPB (Oxford). 2019. In press.

Van Beijsterveld CA, Bongers BC, Den Dulk M, Dejong CH, Van Meeteren NL. Personalized community-based prehabilitation in a high-risk surgical patient opting for pylorus-preserving pancreaticoduodenectomy: a case report. Physiother Theory Pract. 2019. In press.

\section{Book chapter}

Punt IM, Bongers BC, Van Beijsterveld CA, Hulzebos HJ, Dronkers JJ, Van Meeteren NL. Surgery: moving people, improving outcomes. In: Geriatrics. Hyderabad, India: Avid Science. 2016. 


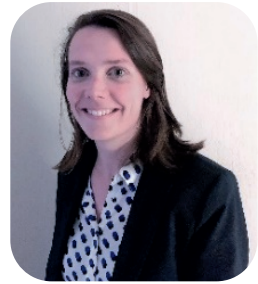

Christel van Beijsterveld was born on the $24^{\text {th }}$ of September 1992 in Breda (the Netherlands). After completing her pre-university education in 2010 at the mgr. Frencken College in Oosterhout (North Brabant, the Netherlands), she studied Rehabilitation Sciences and Physiotherapy at the Catholic University of Leuven (KU Leuven), in Leuven (Belgium). Three years later,

Christel obtained her bachelor degree and continued with a 2-year research master Rehabilitation Sciences and Physiotherapy with a major in Rehabilitation in Internal Disorders at the same institution. She followed her master courses across the five universities of Belgium (Leuven, Antwerp, Ghent, Brussels, and Hasselt) and completed her clinical internships at the academic hospital Leuven (UZ Leuven) and other hospitals and rehabilitation centers in Belgium and the Netherlands.

After obtaining her master's degree (magna cum laude) in 2015, Christel started her PhD project at the Department of Epidemiology in the research line Functioning, Participation, and Rehabilitation within the Care and Public Health Research Institute (CAPHRI) at Maastricht University. She combined her PhD project with working as a physical therapist at the Maastricht University Medical Center+ (Maastricht UMC+). She integrated her research project and scientific knowledge within her daily clinical practice as a physical therapist, acting as an embedded scientist.

Christel received two awards during her PhD project. She was awarded for the "best poster presentation" in 2015 at the national conference 'Onderzoek in Beweging', in Maastricht. In 2016, she won the Dutch Hacking Health Maastricht and received the customer care award from the Maastricht UMC+ board of directors with her idea of the FIT+ application, nowadays called the Hospital Fit App. Moreover, after being a finalist in the PhD action research competition in 2017 , she initiated the Maastricht Platform for Community-Engaged Research (MPCER) with the other finalists. MPCER is a multidisciplinary platform at Maastricht University for individuals who perform, support, or are interested in performing community-engaged research, and thereby intend to have an impact on society by deploying research to resolve societal challenges.

In February 2019, Christel started with the executive master program in Business Administration with a major in strategic management at the University of Amsterdam. Currently, she is working as an embedded scientist and provides project management at the Department of Physical Therapy of the Maastricht UMC+. 
NOTES 

